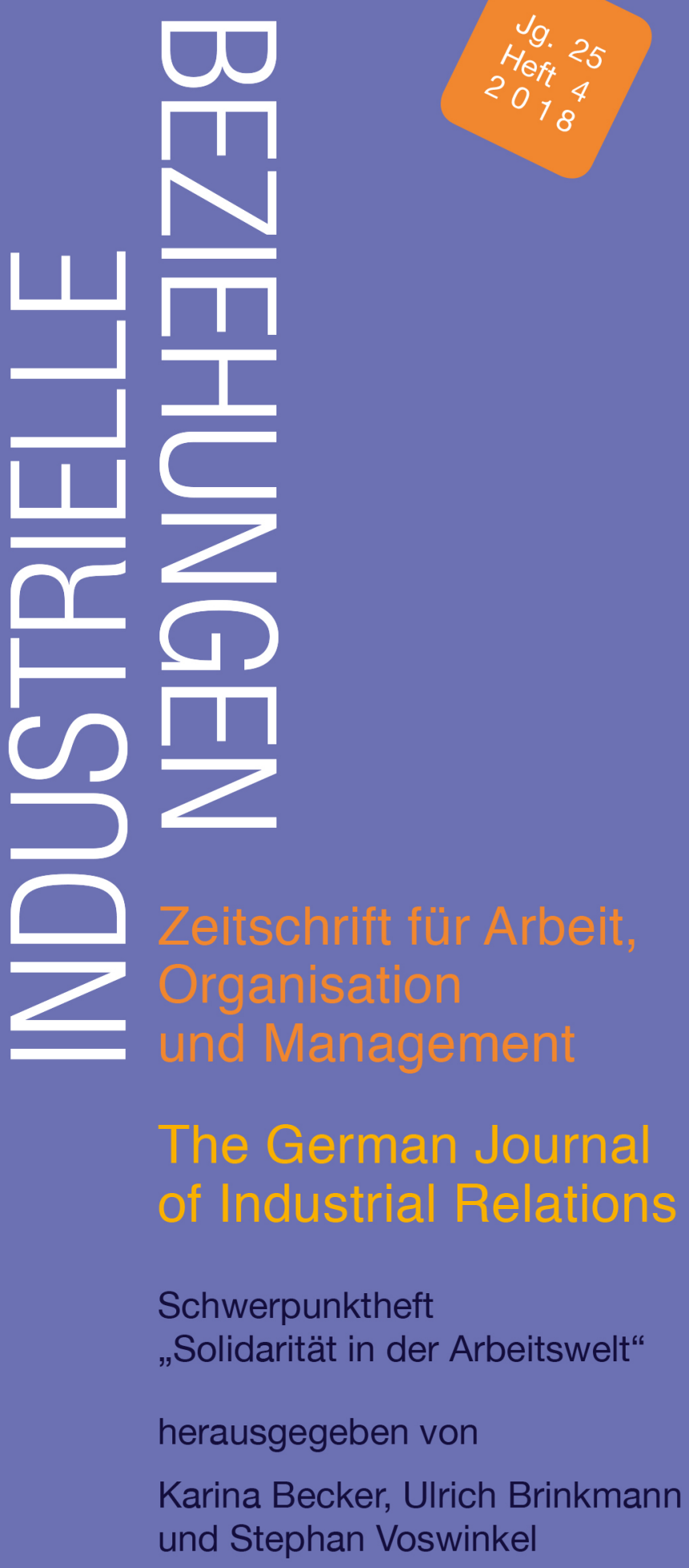

B: Verlag Barbara Budrich 


\section{Inhalt}

\section{Schwerpunktheft "Solidarität in der Arbeitswelt"}

Karina Becker, Ulrich Brinkmann, Stephan Voswinkel

Editorial

Nora Lohmeyer, Elke Schüßler, Markus Helfen

Kann Solidarität „,von unten“ in globalen Zuliefererketten organisiert werden?

Der Fall ExChains

Martin Seeliger

Die soziale Konstruktion internationaler Solidarität.

Gewerkschaftspolitische Positionsbildung im Bereich

der Dienstleistungsfreiheit

Klaus Kock, Edelgard Kutzner

Arbeit als kollegiales Handeln - Praktiken von Solidarität und Konkurrenz am

Arbeitsplatz

Daniel Behruzi

Kampfmethode Ultimatum. Von disziplinierender Kollegialität zu widerständiger

Solidarität - Fallbeispiele aus dem Gesundheitswesen 
Horan Lee, Ronald Staples

Digitale Solidarität unter Arbeitnehmer*innen

Call for Papers: ILERA European Congress 2019

Perspectives of Employment Relations in Europe, Düsseldorf, Germany,

5-7 September 2019

Vorschau 2019 und Dank an die GutachterInnen 2018

Jahresinhaltsverzeichnis 2018 


\section{Karina Becker, Ulrich Brinkmann, Stephan Voswinkel}

\section{Editorial}

„Zweifellos kann die Gesellschaft nicht existieren, wenn ihre Teile nicht solidarisch sind.“

(Emile Durkheim)

Solidarität ist zweifellos ein, wenn nicht der normative Kern der Gewerkschaftsbewegung und die Institutionen der Industriellen Beziehungen (Koalitionsfreiheit, Tarifvertrag, betriebliche Interessenvertretung, Sozialstaat) sind ohne Bezug auf sie nicht vorstellbar. Man könnte sich darüber wundern, dass erst jetzt ein Heft in Angriff genommen wird, das sich schwerpunktmäßig mit Solidarität in der Arbeitswelt beschäftigt. Wir meinen, dass dieses Schwerpunktheft gerade jetzt zur rechten Zeit erscheint und zwar deshalb, weil Solidarität auch und gerade im Feld der Industriellen Beziehungen umstritten und gefährdet ist und hinsichtlich der Frage, was Solidarität in der Praxis ausmacht und einschließt, alles andere als einhellig beantwortet wird. Schon seit längerem kennzeichnen Individualisierung, EntKollektivierung, Exklusions- und Abgrenzungstendenzen das Forschungsfeld; parallel dazu lassen sich neue, translokale Formen von Solidarität beobachten. Vor diesem Hintergrund ist die wissenschaftliche Beschäftigung mit Solidarität nötig, überfällig, aber auch herausfordernd.

Nicht nur in der Arbeiter- und Gewerkschaftsbewegung ist Solidarität ein zentraler Begriff. Auch die Soziologie ist ohne sie kaum zu denken: Der Klassiker Emile Durkheim (1992/1930) ist auch heute noch eine wichtige Referenz für viele Soziolog*innen. Die Katholische Soziallehre beruft sich wesentlich auf sie (Große Kracht, 2017), die Forschung über den Sozial- bzw. Wohlfahrtsstaat kommt ohne sie nicht aus (Kaufmann, 2002; Dallinger, 2009). Selbst Theorien rationaler Wahl stellen sich (in anderer Terminologie) die Frage, wie Kollektivgüter unter Bedingungen allseitigen individuell-nutzenorientierten „rationalen“ Handelns zustande kommen können (Olson, 1965) und beantworten dies auch damit, dass die Voraussetzung funktionierender (Gruppen-)Solidarität das nachhaltige Verfolgen von „self-interest“ sei (Hechter, 1987; Axelrod, 1984; zur Kritik vgl. Smith \& Sorrell,

* Dr. Karina Becker, Friedrich-Schiller-Universität Jena, Kolleg „Postwachstumsgesellschaften“, Humboldtstraße 34, 07743 Jena. E-Mail: karina.becker@uni-jena.de

Prof. Dr. Ulrich Brinkmann, Technische Universität Darmstadt, Institut für Soziologie, Dolivostraße 15, 64293 Darmstadt. E-Mail: brinkmann@ifs.tu-darmstadt.de

PD Dr. Stephan Voswinkel, Institut für Sozialforschung Frankfurt a. M., Senckenberganlage 26, 60325

Frankfurt a. M. E-Mail: voswinkel@em.uni-frankfurt.de 
2014). Dies erinnert wiederum an Adam Smith, der nicht nur der Begründer des EigennutzArguments ist, sondern sich in der „Theory of Moral Sentiment“" auch mit zwischenmenschlicher „sympathy“ und ihrem Beitrag für eine bessere Gesellschaft auseinandergesetzt hat.

Angesichts dieser vielfältigen theoretischen und disziplinären Bezüge erstaunt die Uneinheitlichkeit und Spannweite dessen nicht, was der Begriff bezeichnet und welchen Stellenwert er in den jeweiligen Kontexten besitzt (Jeffries, 2014). In der Theorie Durkheims ist Solidarität eine Bezeichnung für das, was gesellschaftliche Integration ermöglicht, und dafür, wie sie sich herstellt. Der Erklärungszusammenhang ist ein funktionaler, allerdings einer, der normativ vermittelt wird. In anderen Theorien, etwa jener von Axel Honneth (1994, S. 148ff.), steht Solidarität für den gemeinsamen Wertbezug, der als Referenz für die Muster der Wertschätzung als einer Form der Anerkennung dient. In Theorien, die gemeinsame Orientierungen und Werte aus der mit der gemeinsamen sozialen Lage verbundenen Interessenstruktur ableiten, meint Solidarität die bewusstseinsmäßige und normative Haltung, die aus der Einsicht in die objektiven Interessen auf Basis gemeinsamer sozialer Lagen resultiert. Protagonist dieses Forschungsstrangs ist Karl Marx, der Solidarität aus gemeinsamen objektiven Klassenlagen ableitete. Im Kontext von antagonistischen Klassentheorien ist Solidarität dann immer auch eine Solidarität gegen; sie ist somit ein Kampfbegriff. Schließlich kann Solidarität auch als etwas verstanden werden, das sich in Institutionen niederschlägt und durch sie stabilisiert wird - dies gilt für Theorien des Sozialstaats. ${ }^{1}$

Gemeinsam ist den angeführten Theoriesträngen das relationale Moment - Solidarität wird als Beziehungskategorie konzipiert; sie bezeichnet eine Beziehung von Orientierungen, etwa Emotionen und Handlungen zwischen Akteuren, sei es auf Basis von Gemeinsamkeiten (der Interessen), von Sympathie und Empathie oder von normativen Verpflichtungen. Wenn sie sich institutionalisiert, kann diese Beziehungsdimension aus dem Bewusstsein geraten. Der brasilianische Sozialpsychologe Luis Galeão versteht Moral als verdinglichte Form der Solidarität ${ }^{2}$, weil hier eine Verpflichtung gegenüber einer Norm an die Stelle der zwischenmenschlichen Verbindung tritt. Ähnlich können Institutionalisierungen von Solidarität etwa in Regelungen und Leistungen des Sozialstaats vergessen lassen, dass es sich hier nicht um verrechtlichte Aspekte eines Systems handelt, sondern dass diesen lebensweltliche Verbindungen zugrunde liegen. Dies kann zur Folge haben, dass solche Institutionalisierungen Solidarität nicht nur stabilisieren, sondern auch schwächen - beispielsweise, wenn man meint, auf tätige Solidarität, wie gewerkschaftliches oder nachbarschaftliches Engagement, verzichten zu können, weil entsprechende Rechtsansprüche verbürgt zu sein scheinen.

In der Gegenwart scheint dem Begriff in Teilen der Gesellschaft etwas Antiquiertes anzuhaften. Er wird verbunden mit den Arbeiterbewegungen aus früherer Zeit oder wird als pompöses Wort verstanden (so etwa von interviewten jungen Erwachsenen in Billmann \& Held, 2013, S. 1982ff.). Diese Abschreibung des Begriffs verbreitete sich in einer Phase (in den 1980er und 1990er Jahren), in der in der Soziologie und der westlichen Kultur Individualisierung und individuelle Autonomie generell orientierungs- und handlungsleitend wurden. Zugleich wurde die Orientierung an Solidarität auch in dieser Zeit in der Soziologie nicht gänzlich verabschiedet. Es wurde vielmehr darüber nachgedacht, welche Bedeu-

1 Vgl. als guten Überblick über die verschiedenen Bedeutungsdimensionen Bayertz (1998) und Zoll (2000).

2 In einem Vortrag im Dezember 2017 in São Paulo. 
tung Solidarität unter den Bedingungen einer individualisierten Gesellschaft noch haben könne. Ausgangspunkt dieser Überlegungen war und ist die Frage, wie Solidarität bzw. solidarisches Handeln auf der Basis geteilter sozialer Lagen gelingen kann, wenn sich die Arbeiter*innenklasse derart ausdifferenziert und sich die Arbeitsbedingungen so individualisieren, dass von einer Gemeinsamkeit der Interessen kaum mehr die Rede sein kann (Beck, 1983). Rational-Choice-Theorien fühlten sich umso mehr berechtigt, Solidarität entweder nur mehr eine prekäre Bedeutung zuzumessen oder ihren Grund in ihren instrumentellen Funktionen für die allgemeine individuelle Interessenverfolgung zu suchen (vgl. hierzu Tranow, 2012).

Zeitgleich - und in Kompensation zu den weitreichenden Individualisierungstheorien entwickelten sich Begründungsmuster dafür, dass gerade auch moderne Gesellschaften solidaritätsbedürftig sind. Solidarität, so die Erkenntnis, könne nicht mehr allein auf gemeinsamen objektiven sozialstrukturellen Lagen beruhen. Stattdessen sei sie als Leistung in Beziehungen und in Form institutioneller Gewährleistungen zu verstehen und müsse auch eine Solidarität mit Akteuren in anderen sozialen Lagen sein (Hondrich \& Koch-Arzberger, 1992). Diese auf Solidarität beruhenden Beziehungen seien begrenzt und punktuell, sie würden nicht die gesamte Person binden, sondern seien aus Nutzen- oder moralischen Erwägungen und Emotionen gewählt (Bayertz, 1998, S. 30ff.). Die in dieser Zeit prominenten sozialphilosophischen Theorien des Kommunitarismus wiesen indes darauf hin, dass Solidarität gemeinschaftlicher Grundlagen bedürfe und nicht nur in universellen Normen begründet liegen könne, sondern republikanische Grundlagen brauche, also einen gemeinsamen Bezug auf Werte, Geschichte und Zugehörigkeiten (Taylor, 1993).

Diese Debatte ist nach einiger Zeit abgeflaut, ohne dass sie entschieden oder auf eine neue Stufe gehoben werden konnte. Sie wurde auch in der Forschung über Industrielle Beziehungen kaum rezipiert. Mit heutigen Augen gelesen, erscheinen manche der kommunitaristischen Argumente problematisch. Auch wenn Autoren wie Charles Taylor und Michael Walzer der politischen Linken zuzurechnen sind, so finden sich doch einige der Argumente in veränderter, nämlich auf Nation und Ethnie eng geführten Form auch bei der Neuen Rechten. Der nun aufgemachte Gegensatz von Kosmopolitismus/Universalismus und (national verstandenem) Kommunitarismus charakterisiert den aktuellen Diskurs. Der Nationalstaat (oder Europa als neue „Nation“) wird als die unverzichtbare Grundlage von Solidarität und Verbundenheit und somit auch von Institutionalisierungen der Solidarität, wie dem Sozialstaat, verstanden (und in der Konsequenz Geflüchteten und Migrant*innen diese Solidarität verweigert). Der Universalismus gerinnt demgegenüber zu einer moralischen Haltung, der sich nicht in solidaritätsstabilisierenden Institutionen niederschlägt - so die beiden Extrempunkte der Debatte (für einen aktuellen Überblick: Becker, Dörre \& Reif-Spirek, 2018).

Durkheims Unterscheidung von mechanischer und organischer Solidarität scheint hierbei immer wieder auf: mechanische als diejenige Form der Solidarität, die auf gemeinsamer Lage und Zugehörigkeit basiert, organische als jene, die auf der wechselseitigen (und, so könnte man ergänzen: Globalisierung der) Arbeitsteilung beruht und die Erfahrung wechselseitiger Angewiesenheit auch zwischen Lohnarbeitenden unterschiedlicher Ethnie und mit unterschiedlichem Staatsbürgerschaftsstatus zum Ausdruck bringt.

Die daran ansetzenden unterschiedlichen Solidaritätspraxen finden ihren Niederschlag in konkreten Arbeitspolitiken und bestimmen die gewerkschaftliche Auseinandersetzung. 
Diskutiert wird der universalistische Anspruch von Solidarität, der sich etwa an der Frage scheidet, ob Solidarität nur bei gleichen und gemeinsamen Interessen entstehen kann; ob sie - und auf welcher Basis - auch zwischen Arbeitnehmer*innengruppen mit unterschiedlichem Beschäftigungsstatus möglich und erforderlich ist (etwa zwischen „Stamm“- und prekär Beschäftigten) und unter Arbeitnehmer*innen unterschiedlicher Ethnien. Studien offenbaren eine Kluft zwischen der gewerkschaftlichen Normativität und Rhetorik (internationaler Solidarität und zwischen allen abhängig Beschäftigten) und praktischer Interessenvertretung, die sich in Form einer „exklusiven Solidarität“ (Dörre, Holst \& Matuschek, 2013, S. 223; Becker, 2015) der Stammbeschäftigten zeigt.

Erstaunlicherweise wird im Diskurs der Industriellen Beziehungen und auch in gewerkschaftlichen Debatten das Leitbild der „Autonomie“ oftmals aufgegriffen. Zwar muss dieser Begriff nicht im Gegensatz zur „Solidarität“ stehen. Gleichwohl lässt sich sein meist individualisierender Bias kaum übersehen. Er findet sich zunächst in Konzepten, die auch die Verantwortung für sich selbst und das eigene Schicksal ins Zentrum stellen. „Autonomie“ ist zumindest soziologisch irreführend, ignoriert dieser Begriff als Leitbild doch die soziale Eingebundenheit und Prägung der Menschen. Natürlich ist Autonomie ein Wert gerade dann, wenn man sie bestimmten Einschränkungen der Lebensmöglichkeiten entgegenstellt. Aber sie kann nur dann aus ihrem individualisierenden Rahmen gelöst werden, wenn sie als soziale Autonomie gefasst wird - als Autonomie in ihrem Kontext, auf Basis sozialer Voraussetzungen, als Autonomie von Gruppen - mithin: als Autonomie in einem Zusammenhang von Solidarität. Dies mitbedacht, mag man vom Leitbild der Autonomie sprechen, doch drängen sich ohne weitere Erläuterungen liberalistische Assoziationen auf.

Wenn der Begriff „Solidarität“ auch weithin als „,veraltet“ empfunden wird, so finden doch Begriffe Verwendung, die ihm verwandt sind und gleiche oder ähnliche Merkmale aufweisen. Das gilt zum Beispiel für „Kollegialität“ (Hürtgen, 2013; vgl. auch Kock \& Kutzner in diesem Heft), für „Reziprozität“, sozialphilosophisch auch für „Gabentausch“ (Adloff \& Mau, 2005), für „Hilfsbereitschaft“, für „,soziales Engagement“ oder für „Empathie“. Gewiss unterscheiden sich diese Begriffe in ihrem Gehalt nicht unwesentlich von dem der „Solidarität“, gleichwohl machen sie deutlich, dass viele der Dimensionen von Solidarität nicht überholt sind. Das zeigt beispielsweise die steigende Streikbereitschaft in den Sozial- und Erziehungsdiensten und das Engagement in der Hilfe für Geflüchtete oder gegen ausbeuterische Beziehungen in den internationalen Wertschöpfungsketten.

Erfreulicherweise ist in neuerer Zeit eine Zunahme von Publikationen zu registrieren, die sich des Themas ,Solidarität“ annehmen. Dies liegt vor allem an drei Entwicklungen. (1) Da ist zunächst die Flüchtlingsthematik, die die Solidaritätsfrage drastisch und nachdrücklich in einer internationalen Perspektive auf die Tagesordnung gesetzt hat (della Porta, 2018; Lahusen \& Grasso, 2018). Desweiteren (2) werden Fragen nach nachhaltiger ökonomischer Entwicklung und gesellschaftlicher Gerechtigkeit in Debatten über eine ,solidarity economy“ (z.B. Matsui \& Ikemoto, 2015) neu thematisiert, Solidarität erscheint in dieser Tradition als Schlüssel zur Lösung von Marktversagen und sozialen Problemen gleichermaßen. Und schließlich hat (3) die sich von den Finanzmärkten ausbreitende langanhaltende Krise des Kapitalismus ihren Niederschlag in der sozialwissenschaftlichen Forschung zu ihren Auswirkungen auf Solidarität zwischen abhängig Beschäftigten gefunden (z.B. Menz et al., 2013). 
Genug Gründe also, sich mit dem Begriff und dem Thema Solidarität in einem Schwerpunktheft der „Industriellen Beziehungen“ gerade heute zu beschäftigen. Das vorliegende Heft will dazu einen Beitrag leisten. Wir sind überzeugt, dass es uns gelungen ist, darin profunde Beiträge zu versammeln.

Dass die Rolle von Solidarität in einer globalen Perspektive vor allem auch die kulturellnormativen Dimensionen des Begriffs in den Blick nehmen muss, arbeiten Nora Lohmeyer, Elke Schüßler und Markus Helfen am Beispiel der Bekleidungsindustrie heraus. In Auseinandersetzung mit dem von Ver.di-Mitgliedern 2002 gegründeten ExChain-Netzwerk bestimmen sie Solidarität als wechselseitige Bindungen zwischen Gruppen von Arbeiter*innen, die auf einem kollektiven Gefühl der Verpflichtung aufbauen und kollektives Handeln unterstützen. Transnationale Arbeitnehmer*innen-Solidarität ist insbesondere herausgefordert, lokale und globale sowie kurz- und langfristige Ziele gleichermaßen zu verfolgen und zwischen diesen zu vermitteln.

Auch Martin Seeliger widmet sich den Problemen internationaler Solidarität. In seinem Aufsatz setzt er sich mit der Herstellung gemeinsamer Positionen von Gewerkschaften auf europäischer Ebene auseinander. Am Beispiel der Dienstleistungsrichtlinie zeigt er, dass eine gemeinsame Position über zunächst schwer vereinbar erscheinende Interessenlagen insbesondere zwischen west-, nord- und osteuropäischen Gewerkschaften hinweg das Ergebnis eines komplizierten Aushandlungsprozesses ist. Hierbei kommt der Etablierung eines Deutungsrahmens (,Soziales Europa“) eine zentrale Bedeutung zu. Solidarität ist damit Resultat eines Konstruktionsprozesses, in dem auch die unterschiedliche Ressourcenausstattung west- und nordeuropäischer Gewerkschaften einerseits, der Gewerkschaften der neueren Mitgliedsländer andererseits wirksam sind.

Klaus Kock und Edelgard Kutzner beschäftigen sich in ihrem Beitrag mit Praktiken zur Herstellung von Kollegialität, die sie als eine Form der Solidarität begreifen. Dabei zeigen sie, dass sich auch aktuell und trotz konkurrenzbedingter Fragmentierungen in Arbeitsprozessen wechselseitige zwischenmenschliche Bindungen und Verpflichtungen ausfindig machen lassen. Dass sich Kollegialität gegen Konkurrenz und konkurrentes Handeln durchsetzt, leiten sie aus der Einsicht ab, dass die Realisierung eigener Ziele von der Realisierung der Ziele anderer abhängig ist und die eigene Handlungsfähigkeit in der Zusammenarbeit und im reziproken Austausch mit anderen erweitert werden kann.

Eine andere Strategie, nämlich jene, Solidarität als Kampfmethode zu begreifen, beschreibt Daniel Behruzi in seinem Beitrag zu den jüngsten Auseinandersetzungen in deutschen Krankenhäusern. Die Kampfform, die er dafür ausmacht, besteht darin, dass Pflegeteams damit drohen, Leistungen zu unterlassen, zu denen sie per Arbeitsvertrag nicht verpflichtet sind. Dafür stellen sie der Klinikleitung ein Ultimatum, in dem sie erklären, ab einem bestimmten Zeitpunkt nicht mehr außerhalb des Dienstplans einzuspringen, um die Patient*innenversorgung aufrechtzuerhalten. Auf diese Weise gelingt es den Beschäftigten, die disziplinierende Wirkung von Kollegialität aufzubrechen und in Produktionsmacht zu überführen.

Horan Lee und Ronald Staples schließlich entwickeln ein Szenario für die Zukunft von gewerkschaftlicher und arbeitsweltlicher Solidarität in Zeiten der Digitalisierung. Form und Charakter der Solidarität - so die Vermutung der Autoren - werden sich ändern, grundsätzlich werde sie aber ihre Funktion als Quelle von Verhandlungsmacht abhängig Beschäftig- 
ter nicht verlieren. Lee/Staples’ Konzept von „digitaler Solidarität“ knüpft dabei explizit an Durkheims differenztheoretische Konzeption an, zeigt aber auch die Schwächen der dichotomen Gegenüberstellung von mechanischer und organischer Solidarität (gerade für die Industriellen Beziehungen) auf und aktualisiert den Ansatz mit macht- und anerkennungstheoretischen Aspekten. Auch wenn die Autoren der Entstehung und Weiterentwicklung digitaler Solidarität optimistisch entgegensehen, verweisen sie auf die Herausforderung, Kooperationsmodi und meritokratische Normen, neu verhandeln zu müssen: Es ist in ihrer Sicht noch eine lange Strecke von traditionalen zu digitalen Solidaritätsformen, von „funktionärslastigen“ Repräsentationmodellen zu „,basisdemokratischen Partizipations- und Diskursangeboten“ und von der organisationalen Hierarchie zu „commons, assemblies, swarms und/oder weak networks".

Als Herausgeber*innen dieses Heftes wollen und können wir nicht verschweigen, dass uns die insgesamt geringe Resonanz auf unseren Call for Papers verwundert hat. Das bezieht sich insbesondere auf die Themen, die - so meinen wir - aufgrund ihrer Aktualität und Brisanz in ein Schwerpunktheft zum Thema Solidarität in der Arbeitswelt gehören könnten. Dazu gehören das schwierige Verhältnis zwischen Stammbeschäftigten und anderen Gruppen (prekär Beschäftigten, Exkludierten, Geflüchteten) in der Interessenvertretung, die Pervertierung von „Solidarität“ im Rahmen einer ausschließenden ,nationalen Solidarität“ und die Bedeutung dieser Entwicklung auch in den Belegschaften und ihren gewerkschaftlichen und betrieblichen Interessenvertretungen sowie schließlich das Thema „exklusive Solidarität“ selbst. Aber auch die Debatte um Möglichkeiten und Formen der Solidarität in einer Kultur der Individualisierung findet sich nicht repräsentiert - um nur einige Beispiele zu nennen.

Was sagt uns das? Wir denken: Umso nötiger dieses Heft. Und umso nötiger die wissenschaftliche und gewerkschaftliche Beschäftigung mit diesem Thema. Immerhin hat die IG Metall den Begriff der „Solidarität“ in diesem Sommer auf ihre Agenda gesetzt - auf dem Zukunftsforum der Gewerkschaft im August 2018 und in der Entwicklung eines Magazins und einer Umfrage zum Verständnis von „Solidarität“. Das Thema ist also weiter oder wieder - da.

\section{Literatur}

Adloff, F. \& Mau, S. (Hrsg.). (2005). Vom Geben und Nehmen. Zur Soziologie der Reziprozität. Frankfurt a.M./New York: Campus.

Axelrod, R. M. (1984). The evolution of cooperation. New York: Basic Books.

Bayertz, K. (1998). Begriff und Problem der Solidarität. In K. Bayertz (Hrsg.), Solidarität (S. 11-53). Frankfurt a.M.: Suhrkamp.

Beck, U. (1983). Jenseits von Stand und Klasse. Soziale Ungleichheiten, gesellschaftliche Individualisierungsprozesse und die Entstehung neuer sozialer Formationen und Identitäten. In R. Kreckel (Hrsg.), Soziale Ungleichheiten (S. 35-74). Göttingen: Schwartz \& Co.

Becker, K. (2015). Macht und Gesundheit. Der informelle Handel um die Vernutzung von Arbeitskraft. Berliner Journal für Soziologie, 25 (1-2), 161-185. doi: 10.1007/s11609-015-0275-xx

Becker, K., Dörre, K. \& Reif-Spirek, P. (Hrsg.). (2018). Arbeiterbewegung von rechts? Ungleichheit - Verteilungskämpfe - populistische Revolte. Campus. Frankfurt a.M./New York: Campus. 
Billmann, L. \& Held, J. (2013). Die Bedeutung von Solidarität für junge Beschäftigte im Dienstleistungsbereich. In L. Billmann \& J. Held (Hrsg.), Solidarität in der Krise (S. 179-209). Wiesbaden: Springer VS. doi: 10.1007/978-3-658-00912-0_9

Dallinger, U. (2009). Die Solidarität der modernen Gesellschaft - Der Diskurs um rationale oder normative Ordnung in Sozialtheorie und Soziologie des Wohlfahrtsstaats. Wiesbaden: SpringerVS.

Della Porta, D. (2018). Solidarity mobilizations in the 'refugee crisis'. London/New York: Macmillan Publishers Limited. doi: 10.1007/978-3-319-71752-4

Dörre, K., Holst, H. \& Matuschek, I. (2013). Zwischen Firmenbewusstsein und Wachstumskritik. Subjektive Grenzen kapitalistischer Landnahmen. In K. Dörre, A. Happ \& I. Matuschek (Hrsg.), Das Gesellschaftsbild der LohnarbeiterInnen (S. 198-261). Hamburg: VSA.

Durkheim, E. (1992/1930). Über soziale Arbeitsteilung. Frankfurt a.M.: Suhrkamp.

Große Kracht, H.-J. (2017). Solidarität und Solidarismus. Bielefeld: transcript. doi: $10.14361 / 9783839441817$

Hechter, M. (1987). Theories of Group Solidarity. Berkeley: University of California Press.

Hondrich, K. O. \& Koch-Arzberger, C. (1992). Solidarität in der modernen Gesellschaft. Frankfurt a.M.: Fischer.

Honneth, A. (1994). Kampf um Anerkennung. Frankfurt a.M.: Suhrkamp.

Hürtgen, S. (2013). Mensch sein auf der Arbeit? Kollegialität als Balance von allgemein-menschlichen und leistungsbezogenen Aspekten von Arbeit. In L. Billmann \& J. Held (Hrsg.), Solidarität in der Krise (S. 237-262). Wiesbaden: Springer VS.

doi: 10.1007/978-3-658-00912-0_11

Jeffries, V. (Hrsg.). (2014). The Palgrave Handbook of Altruism, Morality, and Social Solidarity. Formulating a Field of Study. New York: Palgrave Macmilan. doi: $10.1057 / 9781137391865$

Kaufmann, F.-X. (2002). Sozialpolitik zwischen Gemeinwohl und Solidarität. In H. Münkler \& K. Fischer (Hrsg.), Gemeinwohl und Gemeinsinn. Rhetoriken und Perspektiven sozial-moralischer Orientierung (S. 19-54). Berlin: Akademie. doi: 10.1515/9783050079677-003

Lahusen, Ch. \& Grasso, M. T. (2018). Solidarity in Europe. Citizens' Responses in Times of Crisis. New York NY: Palgrave Macmilan. doi: 10.1007/978-3-319-73335-7

Matsui, N. \& Yukio I. (Hrsg.). (2015). Solidarity Economy and Social Business. New Models for a New Society. Tokyo, Heidelberg, New York: Springer, Japan.

Menz, W., Detje, R., Nies, S. \& Sauer, D. (2013). Verriegelte Verhältnisse - Solidarität und interessenpolitische Handlungsorientierungen unter Krisenbedingungen. In L. Billmann \& J. Held (Hrsg.), Solidarität in der Krise. Gesellschaftliche, soziale und individuelle Voraussetzungen solidarischer Praxis (S. 31-52). Wiesbaden: Springer VS. doi: 10.1007/978-3-658-00912-0_2

Olson, M. (1965). The Logic of Collective Action: Public Goods and the Theory of Groups. Cambridge/Mass.: Havard University Press.

Smith, Ch. \& Katherine S. (2014). On Social Solidarity. In V. Jeffries (Hrsg.), The Palgrave Handbook of Altruism, Morality, and Social Solidarity. Formulating a Field of Study (S. 219-247). New York: Palgrave Macmilan. doi: 10.1057/9781137391865_10

Taylor, Ch. (1993). Aneinander vorbei: Die Debatte zwischen Liberalismus und Kommunitarismus. In A. Honneth (Hrsg.), Kommunitarismus. Eine Debatte über die moralischen Grundlagen moderner Gesellschaften (S. 103-130). Frankfurt a.M., New York: Campus.

Tranow, U. (2012). Das Konzept der Solidarität. Handlungstheoretische Fundierung eines soziologischen Schlüsselbegriffs. Wiesbaden: Springer VS. doi: 10.1007/978-3-531-93370-2

Zoll, R. (2000). Was ist Solidarität heute? Frankfurt a.M.: Suhrkamp. 


\section{Nora Lohmeyer, Elke Schüßler, Markus Helfen}

\section{Kann Solidarität "von unten" in globalen Zuliefererketten organisiert werden? Der Fall ExChains*}

\section{Zusammenfassung}

Globale Lieferketten in der Bekleidungsindustrie sind durch Arbeitsstandardverletzungen in ihren Produktionsbetrieben, aber auch zunehmend im Einzelhandel gekennzeichnet. Vor diesem Hintergrund ist es wichtig, die Verhandlungsmacht von Arbeitnehmerinnen und Arbeitnehmern entlang der Lieferkette zu stärken. Ein Weg, dies zu erreichen, ist die Schaffung von transnationalen Beziehungen zwischen Arbeiterinnen und Arbeitern. Basierend auf der bestehenden Literatur zu transnationaler Solidarität hebt dieser Artikel die spezifischen Herausforderungen des Verständnisses von Solidarität im transnationalen Raum am Beispiel der globalen Bekleidungskette hervor. Dabei wird versucht, über „Solidarität“ als bloße Metapher für jegliche Form der transnationalen Zusammenarbeit zwischen Gewerkschaften und Arbeitnehmerinnen und Arbeitnehmern hinauszugehen und stattdessen die kulturell-normativen Dimensionen des Solidaritätsbegriffs als gegenseitige Bindung von Arbeiterinnen und Arbeitern zu verstehen. Am Fall des ExChains Netzwerks werden die Möglichkeiten und Herausforderungen der Schaffung und Aufrechterhaltung transnationaler Solidaritätsbeziehungen beispielhaft untersucht. Abschließend werden das transformative Potential, aber auch die Grenzen transnationaler Arbeiterinnen- und Arbeiter-Solidarität hinsichtlich der Arbeitsbedingungen in globalen Lieferketten diskutiert.

Schlagwörter: Arbeit, Zwangsarbeit, Arbeitsdiskriminierung, Arbeitsbedingungen

* Dr. Nora Lohmeyer, wissenschaftliche Mitarbeiterin im Garment Supply Chain Governance Project, Freie Universität Berlin. E-Mail: nora.lohmeyer@fu-berlin.de

Prof. Dr. Elke Schüßler, Professorin für Betriebswirtschaftslehre und Vorständin des Instituts für Organisation, Johannes Kepler Universität Linz. E-Mail: elke.schuessler@jku.at

Prof. Dr. Markus Helfen, Professor für Betriebswirtschaftslehre mit dem Schwerpunkt Personalpolitik, Institut für Organisation und Lernen, Universität Innsbruck. E-Mail: markus.helfen@uibk.ac.at

** Artikel eingegangen: 15.05.2018, revidierte Fassung akzeptiert nach doppelt-blindem Begutachtungsverfahren: 10.09.2018 


\title{
Can solidarity be organized "from below" in global supply chains? The case of ExChains
}

\begin{abstract}
Global supply chains in the garment industry are marked by labour standard violations in factories as well as retail stores. Against this background it is important to strengthen the bargaining power of workers along the supply chain. Establishing direct relationships among workers along the supply chain could be one way to achieve this aim. This paper builds on extant literature on transnational solidarity and highlights the specific challenges of understanding solidarity in a transnational social space by looking at the empirical context of global garment supply chains. It hereby seeks to go beyond treating "solidarity" as a mere metaphor for any form of transnational union or worker cooperation, and instead engages with the cultural-normative dimensions of the concept as referring to mutual bonds among groups of workers. By looking at the case of the ExChains network, this paper examines some of the opportunities and challenges involved in establishing and maintaining transnational worker solidarity. The paper concludes by discussing the transformative potential, but also the limits of transnational labour solidarity regarding substandard working conditions in global supply chains.
\end{abstract}

Keywords: Labor, coercive labor markets, labor discrimination, labor standards (JEL: J47, J5, J7, J8)

\section{Introduction}

Global supply chains in the garment industry are marked by labour standard violations not only in supplier factories typically in the "Global South", but also increasingly in retail stores in the "Global North". Workers in garment production often suffer from excessive overtime, low wages, and various forms of harassment and abuse in addition to potentially deadly health and safety risks (e.g. Schüßler, Frenkel \& Wright, 2018). Often, collective representation to establish bargaining power is made difficult by adverse regulation and aggressive anti-unionism in the countries where production is located. But also garment retail workers-although usually not risking their lives at work-suffer from the comparatively poor working conditions in the retail sector relative to other sectors (e.g. Anner, 2015). In retail, the often female part-time workers with a migration background face severe constraints for effective collective action and are subject to high demands for working time flexibility, wages below the national minimum, an intensification of work, verbal abuse, and various forms of managerial control and surveillance (Appelbaum \& Schmitt, 2009; Grugulis \& Bozkurt, 2011; Köhnen, 2006; Wirth, 2016). Garment retailers and brands in particular have previously been described as being hostile towards unionization and worker representation (Geppert, Williams \& Wortmann, 2015; Geppert \& Pastuh, 2017).

Numerous scholars from diverse backgrounds have argued that transnational labour collaboration is needed to counter unilateral management power in internationally segmented and dispersed global supply chains (e.g. Anner, 2000; 2009; Gordon \& Turner, 2000; Juravich, 2007; Stevis \& Boswell, 2007; Croucher \& Cotton, 2009; Bieler \& Lindberg, 2011). This literature agrees that transnational labour relations need to go beyond single campaigns or initiatives because effective pressure on multinational corporations (MNCs) to improve labour standards ultimately needs to be grounded in well-coordinated cooperation 
and collaboration among unions and their potential allies (Luterbacher, Prosser \& Papadakis, 2017). However, such coordination and collaboration is difficult to establish for reasons like divergent interests and structural differences between unions in the core and the periphery of supply chains, power struggles among unions, exclusive union strategies as well as the lack of resources for transnational activities of trade unions and their global federations (see e.g. Bieler \& Erne, 2014 for transnational union activism and its limits in Europe). Not surprisingly, in the face of these challenges, the literature is mostly sceptical regarding the potential for transnational mobilizing and organizing of workers in global production and retail alike (e.g. Burawoy, 2010; Frege \& Kelly, 2004; Gennard \& Newsome, 2005; Greer, Ciupijus \& Lillie, 2013).

In this paper, we argue that the literature tends to analytically equate coordination and collaboration among unions and union federations with the idea of transnational solidarity. It hereby overlooks the cultural-normative dimension of effective union coordination and collaboration that builds on direct worker interaction and mobilization "on the ground". Against this background, we examine more closely the barriers and possibilities for strengthening solidarity among workers as the normative-cultural glue of transnational labour coordination and collaboration by empirically examining a "grassroots initiative" aimed at connecting workers in different positions in global supply chains: the ExChains network in the global garment industry. ExChains was founded by members of the German trade union Ver.di in 2002 with the aim to build transnational solidarity among workers in garment production and retail by raising issues such as better fire and work protection, higher wages, trade union access rights and supplier transparency from the bottom up. It is a particularly interesting case for studying transnational aspects of solidarity, as it allows us to examine how traditional notions of craft or class solidarity (Heckscher \& McCarthy, 2014) extend to or need to be refined in the new context of global production. In this effort, we refrain from treating "solidarity" as a mere metaphor for any form of transnational union cooperation or collaboration. Instead, we engage directly with solidarity as the cultural-normative dimension of labour cooperation and collaboration (also Fantasia, 1988; 1995), defining solidarity as the mutual bonds among groups of workers that are built around a communal sense of obligation to support collective action.

In what follows, we critically examine the previous literature on transnational solidarity and discuss the problems of a structurally over-determined view on transnational labour collaboration and labour power. We then examine the ExChains network as an illustrative case of the opportunities and challenges of establishing and maintaining transnational solidarity among workers. We conclude by discussing the transformative potential of transnational labour solidarity of this sort with regards to substandard working conditions.

\section{Transnational solidarity in global supply chains}

The concept of solidarity - as distinguished from more structural aspects of unions' collective action (Heckscher \& McCarthy, 2014) - adds an understanding of the culturalnormative dimension of structural, associational, institutional, and societal power of workers. In the employment relations literature, worker solidarity is widely regarded as a basic 
condition for collective action on behalf of workers, particularly concerning the formation of unions, the staging of industrial action and collective bargaining (Doellgast, Lillie \& Pulignano, 2018). In the theory of collective action, solidarity is seen as more important for workers than for employers, because workers are weaker in power and resources. For example, Offe and Wiesenthal (1980, p. 78) explained "(...) that those in the inferior power position can increase their potential for change only by overcoming the comparatively higher costs of collective action by changing the standards according to which these costs are subjectively estimated within their own collectivity". Similarly, Hyman (2001) considers the strategic construction of solidarity as one of the main purposes of trade unions. In a more recent contribution, Tapia (2013, p. 671), looking into union re-vitalizing strategies by leaning towards social movement type of activism, emphasizes a "relational culture," that is, a "culture based on fostering relationships, leading to high levels of trust and loyalty towards other members and the organization" as fostering commitment. And analyzing successful organizing attempts in the UK entertainment and higher education sector, Simms and Dean (2015) identify the capacity of organizers to frame collective interests as "cultures of solidarity" (Fantasia, 1988) as a key ingredient for evoking collective action.

Nevertheless, solidarity is rarely explicitly theorized as an ideational foundation of collective action and thus, potentially, as part of an independent power resource of workers (for a prominent exception s. Fantasia, 1988; 1995). In other words, solidarity might not just be a pillar of structural power resources (cf. e.g. Schmalz \& Dörre, 2014), but might also foster the ideational power of workers especially in situations where structural sources of power are weak, as in the context of most global supply chains. ${ }^{1}$ While the concept of international or transnational solidarity is receiving increased attention in the literature (e.g. Pernicka \& Glassner, 2014; Pernicka, Glassner, Dittmar, Mrozowicki \& Maciejewska, 2017), the specific context conditions of global supply chains are rarely explicitly addressed. Here, transnational forms of solidarity are particularly hard to construct, because two traditional pillars of solidarity more or less present within a given workplace or country-daily social relations and locally shared ideologies or causes (Heckscher \& McCarthy, 2014) — are usually absent. Thus, solidarity needs to be actively created through organizing for shared experiences and identifying common reference points among workers in these settings.

\subsection{Structural obstacles to trade union power in global supply chains}

As workers across the world are put in systematic interconnection through global supply chains, their shared experiences of economic exploitation could be seen as a potential enabler of links of transnational labour coordination and collaboration. However, workers along the supply chain are socially, culturally and spatially separated from each other, have few direct personal connections, and their individual economic incentives contradict each other (but see Sassen, 2012 on connections among activists via electronic links). Hence, the coverage of labour standards is typically observed as being decreasing with every step "upstream" the value creating processes, especially in the smaller, usually subcontracted factories in the Southern parts of global supply (Grimshaw, Marchington, Rubery \& Willmott, 2005; Levy, 2008; Palpacuer, 2008). But also “downstream", in the retail or logistics and

1 Other dimensions of ideational power resources might be shared knowledge, traditions or customs. 
service functions of global buyers', labour standards are deteriorating due to outsourcing and other flexibility measures.

The unilateral response of multinationals to this problem, most commonly in the form of firm-level codes of conduct drafted in response to pressure from external stakeholders, is widely considered as insufficient (Barrientos \& Smith, 2007; Locke, Amengual \& Mangla, 2009; Lund-Thomsen \& Lindgreen, 2014). While a transnational "texture" of labour-related norms and regulations might be emerging (Pries \& Seeliger, 2013), local union power and related collective action capacities are still weak in the-both geographic and organizational-peripheries of global supply chains due to outsourcing dynamics within Western capitalism and offshoring to countries with weaker or weakly enforced labour laws (e.g. Bieler, Lindberg \& Pillay, 2008). Likewise, there is widespread scepticism about the capacity and willingness of governments to enforce the global standards of the ILO given their belief in benefitting from regime competition through lowering labour costs (Anner, 2015).

Hence, unions and union federations have increasingly become aware of transnational union coordination and collaboration as a prerequisite for organizing countervailing power in the transnational arenas of global supply chains spanning across several national industrial relations (IR) systems (e.g. Fichter, Ludwig, Schmalz, Schulz \& Steinfeldt, 2018; Helfen \& Fichter, 2013). At the same time, however, the problem of employment and working conditions below the ILO standards in global supply chains is fuelled by severe structural obstacles for workers to organize into unions. Using the power resource framework we see structural, associational, institutional, and societal power as weak in the global garment industry. Similar to Crozier and Friedberg's (1979) conceptualization of power in organizations, the literature on trade union power has distinguished structural power resources, i.e. power derived from workers' location and role in a production system, from associational power resources, i.e. power related to unions' collective action capacities resulting from their membership and organizational infrastructure (Jürgens, 1984; Wright, 2000; Silver 2003). Furthermore, continental European scholars have introduced institutional power resources, which refer to the enforceable rights and duties of unions and their resulting social acceptance (Dörre, Holst \& Nachtwey, 2009; Brinkmann, Choi, Detje, Dörre, Holst, Karakayali \& Schmalstieg, 2008; Schmalz \& Dörre, 2014) as well as societal power, i.e. a union's capacity to develop influence outside the workplace through cooperating with and gathering support from other societal actors (e.g. churches, NGOs, political parties) (Schmalz \& Dörre, 2014).

Regarding structural power, in garment production the "thousands of relatively small firms with minimal sunk costs in any given location and mobile equipment (sewing machines)" together with possibilities for subcontracting, provide lead firms with "the ability rapidly to shift the location of work at little cost" (Anner, Greer, Hauptmeier, Lillie \& Winchester, 2004, p. 20). This mobility of capital—often expressed in notions of 'spatial fix', 'regime shopping' or 'race to the bottom'-stands in stark contrast to the local dependence of labour ('immobility'), a problem that is often seen as being at the roots of worsening working conditions (Anner, 2015; Merk, 2009). The relative abundance of workers with low resource endowments and a lack of alternative employment options-especially in the producing countries-weakens the structural power of workers vis-à-vis employers (Brookes, 2013; Hardy, Calveley, Kubisa \& Shelley, 2015). But also the retail sector in the 
US and Europe relies heavily on women and young workers, often with a migration background, who are employed with a minimum of fixed hours and for low wages (Appelbaum $\&$ Schmitt, 2009). These workers have equally limited employment alternatives and low bargaining power. In the transnational space, the fragmented nature of global supply chains undermines the usage of single hotspots as levers for union influence. Unions in this context tend to apply exclusive strategies, i.e. they focus on representing shrinking core membership rather than working towards solidarity across workplaces, skill levels and ethnicities (Doellgast, Lillie \& Pulignano, 2018).

The associational power in garment production and retail is weak, too. As most garment manufacturing has been subcontracted and offshored, the bulk of employment in Western economies is within retail groups and some niche garment and textile production (Bosch \& Lehndorff, 2004). Some of the large retailers in the West are known to be rather adversarial towards unions and works councils and have cancelled collective agreements (Geppert, Williams \& Wortmann, 2015). Frequent dismissals of works council members in this sector provide obstacles to the organization of retail workers (e.g. Köhnen, 2006). In supplier countries like Cambodia or Bangladesh, the number of unions has been increasing (Oka, 2016; Labowitz \& Baumann-Pauly, 2015). However, this development is not necessarily a sign of greater associational power. On the contrary, as Oka (2016, p. 664) reports for Cambodia, the growth in the number of unions has led to increased competition between unions, compromising 'unions' effectiveness in promoting collective bargaining and broader pro-worker agenda as they compete for members and obstruct each other's activities." Moreover, the fragmented nature of global supply chains again leaves national unions at a strategic disadvantage (Fairbrother \& Hammer, 2005).

Although the institutional power of retail unions has been eroding more or less intensely in different national capitalisms, institutional power is particularly weak in producing countries in the Global South (e.g. Brookes, 2013). In countries like Bangladesh or Cambodia, governments are either not able or not willing to implement labour laws (e.g. Mosley, 2017). Furthermore, the strong interrelationship between the state and employers particularly in Bangladesh reinforces the oppression of unions, resulting into a labour control regime that Anner (2015) called 'market despotism.' For example, a 30\% threshold for union formation as well as continued intimidation of workers when they try to register to a union places a high burden on unions to lever their formally granted institutional power.

In countries like Bangladesh, where unions are usually portrayed as hampering economic growth and prosperity, also the societal power of unions can be expected to be rather weak. When attitudes towards unions are relatively sceptical - or workers have limited knowledge about unions and often even lack a clear worker identity (Kabeer \& Mahmud, 2018) - it is unlikely that unions will be able to establish cooperative relationships with other societal groups or that their demands will be seen as encompassing broader societal concerns, although ties particularly to international NGOs are strengthening (e.g. Merk, 2009). Bieler (2012) lists a number of promising efforts through which organized labour has been able to reach beyond its immediate constituencies. For instance, the overarching "Labour and Globalisation Network" promotes a broader understanding of workers and workplaces that is more suited to the globalized economy and encourages cooperation between unions and social movements in the sphere of social reproduction and the environment. Digital technology is seen as an enabler of 
this new type of labour movement. However, strong competition between different unions and between unions and social movements has so far prevented transnational solidarity and joint action. While rightly seen as an important power resource to promote union's issues, such relationships can be seen as limited when it comes to organizing, i.e. strengthening union work itself rather than unions' causes.

Despite these structural barriers, different forms of cross-border union organization are emerging, which we will review below. Additionally, it has been argued that the changing temporality of global supply chains with an increased focus on speed and just-in-time production might enhance the structural positions of workers and create new spaces for resistance (Fichter et al., 2018; Herod, 2000). While we see little changes in this direction in the still intensely buyer-driven garment supply chains (Gereffi, 1994), strategies for organizing and managing workers might indeed be changing in producer-driven ones (see Helfen, Schüßler \& Sydow, 2018).

\subsection{Prevalent forms of transnational unionism and their limits regarding transnational worker solidarity}

Most of the literature on transnational unionism focuses on extant institutions and organizations which are sometimes equated with transnational solidarity. For example, as for the European Union, European Works Councils are emphasized as a space in which transnational solidarity might be constructed (e.g. Pernicka \& Glassner, 2014; Pernicka et al., 2017; Kotthoff \& Whithall, 2014). In the more limited literature on global supply chains, the focus is mostly on global union federations (GUFs) and how they shape the collaboration among unions across different countries in the spirit of solidarity through various practices. Here the most common organizational practices of cross-border union cooperation and coordination are international campaigning, GFAs, and international accords.

First, (inter-)national campaigns seek to establish external pressure in cases of acute labour violations. Especially in "the garment industry, the past 15 years have seen numerous such grassroots social struggles, in which workers have successfully mobilised allies overseas to support them" (Merk, 2009, p. 606). These campaigns are mostly organized by international NGOs, often focusing on consumers 'buying power' (Donaghey, Reinecke, Niforou \& Lawson, 2014) in order to tackle working conditions and substandard wages in producing countries. Campaigns, such as those organized by the Clean Clothes Campaign, are said to "build labour solidarity across space" (Merk, 2009, p. 600). While especially international campaigns have been effective in raising awareness for weak labour standards, they are not targeted at unionizing workers. Often, workers remain in the role of information-providers (e.g. Bieler, 2012, p. 371) thereby reproducing, if not reinforcing, already existing power asymmetries (Featherstone, 2012). Additionally, the temporary nature of these campaigns lends itself to focusing more on short-term goals rather than on establishing continuous and sustainable forms of countervailing power. Power of workers is 'borrowed' from international partners and NGOs, which follow their own campaign logic. Thus, while international campaigns expand the reach of unions into society and are therefore fostering societal power, they are limited in supporting structural, associational or institutional power of workers and their representatives, particularly in the "Global South". 
Second, with the enormous expansion of transnational corporations (TNCs) in the process of economic globalization the international union movement reorganized the International Trade Secretariats (ITS) into GUFs, giving greater priority to union organizing and negotiating activities vis-a-vis TNCs than to lobbying international institutions (ILO). In spite of other attempts of trade unions to (re-)gain influence on the global organization of production such as European works councils, transnational wage coordination or cross-border union alliances (e.g. Müller, Platzer \& Rüb, 2006; Levesque \& Murray, 2010), GUFs have identified global framework agreements (GFAs) as a strategic tool for establishing a cross-border framework of recognized norms, principles and procedures with MNCs that is independent from and supplemental to national-level bargaining and legal provisions (Papadakis, 2008). For the GUFs, GFAs are a means of securing trade union recognition, providing space for organizing and influencing employment practices of MNCs within their global supply chains (Croucher \& Cotton, 2009; Hyman, 2005; Platzer \& Müller, 2009; Stevis \& Boswell, 2007). GFAs, while effective in establishing certain boundary conditions in labour-management relations, cannot directly affect local union membership and local union power in supplier countries (Helfen \& Fichter, 2013). However, GFAs do have the potential to enhance the associational power of unions in producing countries in that they often explicitly endorse the right to collective bargaining as specified by the ILO. To implement this right in adversarial contexts, GFAs rely on associational, institutional, and societal union power in MNC host countries (Helfen, Schüßler \& Stevis, 2016). GFAs, thus, might support the structural, associational, institutional or societal power of local unions through transnational solidarity between unions in MNC's host countries and worker representatives in supplier countries.

Third, as an extension of GFAs, international accords comprise multiple brands (Alexander, Ashwin, Lohmeyer, Oka \& Schüßler, 2017) and hold them accountable through binding agreements with GUFs, typically on the basis of a multi-stakeholder dialogue involving NGOs as well. The Bangladesh Accord is the prime exemplar of such an agreement and is seen by many as a promising opportunity for workers in despotic' regimes of labour market control (Anner, 2015; Reinecke \& Donaghey, 2015; Schüßler et al., 2018). Again, however, the Accord remains limited in its potential to strengthen workers' structural, associational and societal power (Baumann-Pauly, Labowitz \& Banerjee, 2015). For instance, "[w]hile union members have the right, under the Accord on Building and Fire Safety, to participate directly in the inspection process, not all factories have union representation" (Mosley, 2017, p. 158). The challenge, thus, remains to link these initiatives to the local level in order to build union power and capacity to act on the factory level. Although generally optimistic regarding the Accord's potential to achieve these aims as Bangladeshi trade unions were able to strengthen their associational, institutional and societal power resources through interactions with workers and international allies, Zajak (2017) also concedes that relying on external support might decrease local unions' internal potential for organizational development, democratic organizing and solidarity-building across unions. Also, the Accord has so far failed to be endorsed by actors outside the factories, with for instance the Bangladeshi government articulating open dissent, and thus is limited in strengthening workers' societal power.

In our view, these forms of transnational unionism express a concern for transnational solidarity but should not be equated with it, because they tend to overlook the ideational 
dimensions of "two-way mutual bonds of solidarity" (Davis, 2017, p. 167) that is experienced reciprocally rather than granted in a one-way direction (Dribbusch, 2014, p. 338). This kind of support, although effective in many ways, often remains "dependent on episodic overlaps of interest and consumer preferences" (Anner et al., 2004, p. 22) and leaves fundamental power asymmetries untouched.

\subsection{Revisiting solidarity as a theoretical concept: Transnational solidarity as an ideational power resource?}

Against the background of unions' structural, associational, institutional, and societal weakness both in the centre and the periphery of global garment supply chains, workers and unions face tremendous in gaining bargaining power vis-à-vis employers (e.g. Bieler, Erne, Golden, Helle, Kjelstadli, Matos \& Stan, 2015). Where global production leaves few structural power resources to workers, their associations and state regulations are weak, and chosen forms of collective action like campaigns, agreements, and dialogue often fall short of producing a lasting impact, it might be justified to examine the ideational and cultural dimension of power resources like knowledge, solidarity, norms or customs. The role of ideas and identity has been largely acknowledged in the literature on union organizations. Trying to understand the challenges of European trade unions, Hyman (2001, p. 173), for instance, argues that "part of the problem is an erosion of credible mobilizing rhetorics, of visions of a better future, of utopias. Building collective solidarity is in part a question of organizational capacity, but just as fundamentally it is part of a battle of ideas." But what is solidarity and how does it relate to the ideational power resources for collective action?

As noted above, from a political economy perspective, solidarity is more important for workers than for employers because they are weaker in power and resources. Two statements can be derived from this condition: solidarity includes a foregoing of individual advantages for the sake of a greater good for a collective; and, if present, solidarity empowers individual workers by correcting resource scarcity in cases of conflict. In contrast to this rather economic definition, Doellgast and colleagues (2018, p. 13) define worker solidarity as "the adherence to principles and patterns of behaviour that support mutual aid and collective action; particularly those that concern labour union strike and bargaining strategy." As a corollary, solidarity as a normative orientation or behavioural disposition of individuals is related to group identity, which is formed from within a group and in relation to other groups (inclusive vs. exclusive). From a social constructivist perspective, however, rather than being taken as a given, Fantasia (1995, p. 280) argued that "cultures of solidarity" or "collective consciousness" form in relation to specific strategic encounters and moments of conflict, so that solidarity is best considered as mobilized and largely independent of collectivized, individual ideas and beliefs.

Like other norms, solidarity can be expected to emerge more easily in smaller groups or in groups regarded as similar (by whatever criterion), because these groups are connected by structurally dense social networks (Grannies, Smith \& Stepan-Norris, 2008). As Tapia (2013, p. 672) writes: "According to social commitment theory, people will develop affective ties to the group (a) when they work together on joint tasks, and (b) when there is a sense of shared responsibility." Similarly, Davis (2017), studying the case of global social movements, argues that a shared sense of mutual oppression is consciously created by ena- 
bling visits among activists and organizing joint experiences of eating, dancing, and living together. The importance of such agency has also been highlighted for unions, e.g. when organizing migrant workers across borders. Here, Hardy (2015) highlights the importance of piecemeal achievements realized through visits of delegations, the set-up of joint websites and the creation of joint experiences through campaigns. "Transient" forms of solidarity can be established using social media (Heckscher \& McCarthy, 2014).

We will define transnational worker solidarity as mutual bonds among workers, building on a communal sense of obligation to support collective action focused on issues that connect to work, but not exclusively to workplace issues (Heckscher \& McCarthy, 2014). Following Fantasia (1988; 1995), we consider transnational solidarity as collective, dynamic, based on cultural practices, collective action, and social organization. Thus, although bearing a strong ideological component, transnational solidarity stems from lived experiences (e.g. shared experiences of substandard working conditions and exploitation) and strategic encounters and conflicts (e.g. organizational restructuring or industrial dislocation) and is situated in specific forms of social organization (e.g. fragmented production or individuated work). In short, solidarity needs to be organized, but is also at the roots of organizing. Understood in this way, transnational solidarity depends on an active facilitation of interactive processes among workers that create shared experiences and a common understanding that norms have been violated (Fantasia, 1988; Doellgast, Lillie \& Pulignano, 2018; Banting \& Kymlicka, 2017; Heckscher \& McCarthy, 2014). Unions play a key role in organizing and framing such activities, but face at least two fundamental dilemmas and tensions.

First, while organizing workers needs long-term, continuous efforts, international campaigns have been criticized to aim for 'quick fixes' rather than long-term developments on the ground. A dilemma thus lies in bridging the long-term goals of unions with the shortterm gains of international campaigns to establish long-term worker power. Transnational strategies of unions often aim for long-term, political interests, which hamper local mobilization for transnational solidarity as they remain distant from the reality of local workers (e.g. Bormann, Jungülsing, Bian, Hartung \& Schubert, 2015, p. 7). However, campaigns in the garment industry (in Guatemala and Haiti), which in the short-term seemed successful, ultimately ended in the closure of the focal factory (see Anner, 2000, p. 248-252). There is, thus, a need to bridge the long-term goals of unions and the short-term gains of international campaigns to establish long-term, yet local and grassroots worker power.

Second, and relatedly, the local and the global level are intrinsically tied together in global supply chains (Anderson, 2015). For getting some ground in countervailing MNCs' activities, workers must therefore organize simultaneously across space as well as in space (Davis, 2017). That is, while counter-movements need to be global (Burawoy, 2010), the local level cannot be dismissed (Caspersz, 2010). There is a need for local actors to 'jump scale' (Merk, 2009) to withstand powerful employers on the transnational level, while simultaneously seeking to establish the necessary effective structures on the ground on which transnational action is dependent. Put differently, while "campaigns with a weak or nonexistent international component will fail or meet with limited success (...), international support can never replace sustained organizing efforts by local actors at the point of production" (Anner, 2000, p. 248, see also Jessup \& Gordon, 2000, 'pressure from below' and 'pressure from above'). 
Successful cases can be found in Europe, where for instance the European Metalworkers' Federation has partly successfully coordinated national-level collective bargaining rounds at a European level (Schulten, 2005, cited in Bieler, 2012). In a global supply chain context, Merk (2010) highlights the Asian Floor Wage initiative as an attempt to establish solidarity between workers and prevent wage competition. The campaign highlights that only through the joint effort of workers can pressure be put on factory owners, buyers, and governments. But, as already highlighted above, several obstacles to transnational union cooperation prevail, such as a continued focus on national contexts, resource constraints, and the absence of cross-country frameworks to support solidarity (Gennard \& Newsome, 2005).

Figure 1 summarizes our discussion and highlights the two tensions that transnational worker solidarity faces. While the local and the global as well as the short-term and the long-term need to be balanced, the arrows show that addressing one part of these tensions also requires addressing the other. Without addressing the short-term, for instance, longterm goals cannot be reached. And without addressing the local level, global solidarity relations are unlikely to emerge.

Figure 1: Tensions in organizing transnational solidarity in global supply chains

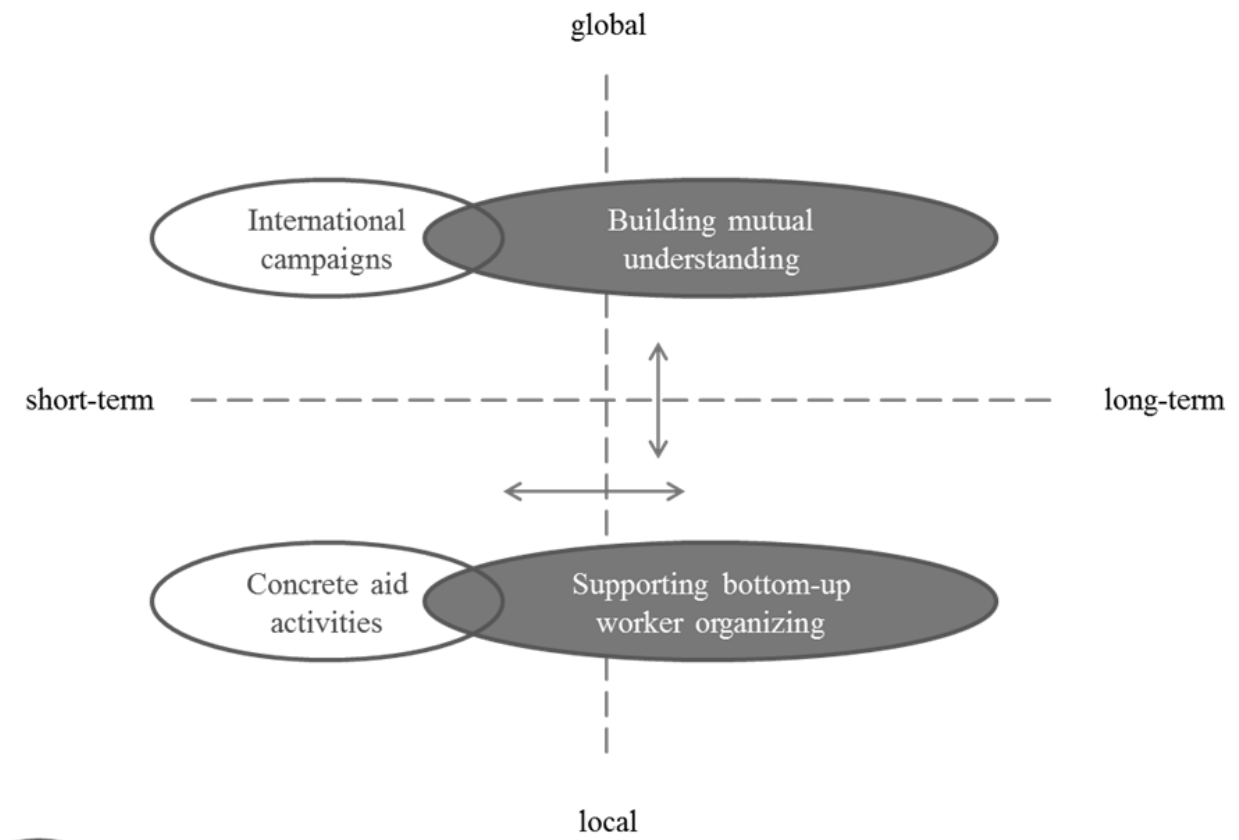

$=$ ExChains' focus 


\section{Methods and Data}

As outlined above, ideational power resources might be particularly important in the context of the global garment industry where structural, associational, institutional, and societal power resources tend to be weak both in the periphery and increasingly also the core of global supply chains. The ExChains initiative in this sector is thus a critical case to examine whether and how transnational solidarity among workers can be organized.

We build our analysis on data from six interviews with unions and union related organizations, 65 documents published by the ExChains network and its members, and field notes from participating in 10 industry events revolving around transnational solidarity issues. We systematically analysed all 81 documents using qualitative data analysis software (NVivo). In a first step we reviewed all documents to get a better understanding of the network's structure, aims and activities. In a second step and following our theoretical framework (see Figure 1) we analysed how the ExChains network approaches the identified tensions (long-term vs. short-term and global vs. local), focusing on the concrete practices through which this is done. This second step involved several rounds of going through the material and discussing as well as revising emerging findings. In the following we introduce the ExChains network and outline its approach to fostering transnational worker solidarity.

Some of the practices we are going to present address both tensions, whereas others are addressing just one of them, although both tensions often overlap. The ExChains network as a critical case

Programmatically and according to their bylaws, all unions of the DGB (Deutscher Gewerkschaftsbund) are supportive of transnational worker solidarity, and are dues-paying members of the respective international organizations, GUFs and European union federations (EUFs). Within that setting, ExChains is a Ver.di-affiliated network of works councillors and union members working along the global garment supply chain-from Asia's garment production factories to European retail stores. ExChains has members from Germany (Ver.di as well as works councillors from H\&M, Zara, Primark, and Esprit), Bangladesh (National Garment Workers Federation (NGWF), Sri Lanka (Free Trade Zones and General Service Employees Union (FTZ\&GSEU)), and India (Garment and Textile Workers Union (GATWU) in Bangalore and Garment and Fashion Workers Union (GAFWU) in Chennai).

The ExChains network exists since 2002 and is part of a wider network called TIE (Transnational Information Exchange). ExChains' financial situation is quite precarious. While the positions of two coordinators in Germany are financed via TIE, which itself is partially financed by the foundation "Menschenwürde und Arbeitswelt" through the Evangelischer Entwicklungsdienst (EED) as well as a private donor from the US who is sympathetic to TIE's goals, the political work-mostly campaigns or meetings-has to be co-financed by third party funders, such as the Bewegungsstiftung or the Rosa Luxemburg Foundation. The networks in Asia are partially funded by the respective unions but have also received funds from foundations such as the Rosa Luxemburg Foundation. There are further sub-networks connected to TIE, which likewise try to organize transnational solidarity, such as the network "Eisenbahn ohne Grenzen," which seeks to organize railway workers from West-Africa and France. Whereas TIE operates in different sectors, ExChains focuses on the global garment industry. The aim of the network is to build transnational solidarity among workers and, ulti- 
mately, to permanently change the global structure of the industry. The issues addressed by ExChains are diverse. In its current campaign, ExChains raises four demands: better fire and work protection, higher wages, trade union access rights, and supplier transparency. We argue that ExChains differs from other transnational initiatives because it decidedly aims to establish two-way bonds of solidarity among workers in garment production and retail.

ExChains tackles the tension between both short and long-term goals as well as the need for both local and global mobilizing and organizing by building on an iterative process of active mobilization on the local level, which enables the worker's engagement for longterm, transnational goals and, ultimately, establish bonds of transnational solidarity. Mobilizing workers for transnational solidarity in the case of ExChains, thus, starts on the local level and is then built upon to establish transnational connections between workers in Bangladesh, India, and Sri Lanka on the one hand, and Germany on the other hand. Mobilizing locally, however, is seen as a continuous process and a goal in itself, rather than 'just' a means for transnational collaboration. It is intended to also build the ground for long-term organizing of workers, both in garment retail and production, and thus the fostering of local union structures.

\subsection{Local/short- and long-term: Direct and active involvement of union members and workers and the creation of shared interests}

One important pillar of this approach, which differs from other transnational initiatives such as international campaigns, GFAs or accords, is to actively and directly involve second-line unionists and workers rather than union officials. While building on established works councils in garment retail and local unions in garment production as well as the relationships between local unions at both ends of the supply chain that have been established in the realm of TIE, ExChains explicitly seeks to involve workers in a 'grass roots' fashion. Rather than the mere participation of workers in forms of otherwise organized collective action, this involves the active participation of workers in identifying issues, formulating interests and demands and planning and organizing campaigns. ExChains thus seeks to involve workers on the shop floor directly and in an active manner.

"... our approach is really to say: well, if we really want change, then we need to empower those that are concerned or, better, they need to empower themselves-which is the better term- to be able to enter into negotiations about collective agreements, ...." (170922). ${ }^{2}$

Reaching such empowerment and involving all workers in the identification and formulation of interests is of course a difficult process, which needs to be actively guided by members of the network:

"There is no such thing as given interests for which you just have to search. Rather there are only interests that you have created for yourself, to say it this way. This means there is a philosophy to sit together and think about: 'What is your problem? What is my problem? Are there any connections? Let's formulate our interests jointly.' This way of thinking is established for years — at least within the strong [unionized] works

2 Where interviews were held in German, the interview quotes were translated from German to English by the authors. To preserve the anonymity of our interviewees, we only provide the date of the interview for identification. 
councils - and this way of thinking of course matches our current idea of international cooperation very well" (170922).

He recounts that the organising strategy-to identify what common interests are and jointly develop strategies for how they can be reached-had been established in German works councils for a long time before being applied in garment factories. ExChains thus seeks to follow a bottom-up approach, allowing workers to address themes that are important to them and actively participate in planning and realizing activities, enabling stronger identification, learning, and experiences of self-efficacy.

A central means through which shared interests among local workers-and, as we will see later, workers along the supply chain - are created are so-called work place or health mappings. Here, groups of workers jointly identify the work-related problems they face (e.g. health issues), which tasks in their work are responsible for causing these problems, and how these affect their private and family lives. The joint mapping helps workers to realize that they all face very similar problems, that these are related to working conditions rather than their individual weakness (as factory managers and supervisors often make them believe), and that their unhealthy working conditions have huge impacts on many parts of their private and family lives. Finally, the mapped causes of health problems are listed and ranked according to their importance. Workers then vote which issues are most severe and should be addressed first, to then jointly develop ways through which these can addressed. Work place mappings facilitate local organizing and mobilization through, first, reflecting on and bringing to light shared problems and accordant interests. Such efforts may also help to establish a form of "worker identity" among garment workers in the first place - often young women that do not envision a long-term trajectory as workers. Second, concrete and practical issues are addressed that can be realized in the short-term and from which workers profit locally, such as negotiating better lighting or air conditioning in the factories or retail stores. These short-term improvements allow for the organization of workers and ultimately strengthen local union power, also in the long-term. One of the workers reports that the experience of factory-level negotiations has encouraged him and his colleagues to fight against social security cuts: "It showed what we can achieve when we stand together. The government eventually had to withdraw the planned cuts. That was a victory for all garment workers." (cited in ExChains, 2016)

In order to maintain the engagement of workers, the network began focusing on getting the workers' families on board as well. Inviting family members to meetings and explaining the importance of unions to them seeks to foster understanding for their family member's engagement despite their long working hours and family duties (180309). This is even more important where unions are regularly de-legitimized by powerful actors, such as the government or industry associations, and where workers engaged in unions face repressions.

The direct involvement of workers as well as the strong focus on local organizing is presented by our interviewees as an explicit response to transnational initiatives, which aim at implementing international labour rights, but often miss to strengthen labour power on the shop floor. Often providing little room for the demands of workers, these internationally organized initiatives thus tend to overemphasize the global scale. As one interviewee reported, the necessity for such a strong focus on strengthening the power of local workers was a learning process within the ExChains network and resulted from the experience that 
allying with international initiatives or NGOs - while positive in many ways - often grants workers not more than a form of - what he called - 'borrowed power': “...one reason for our quite fundamental strategic reorientation, but at the same time criticism of our previous practice, is that often these campaigns do not lead to the development of counter-power on the ground..." (170824). It was also reported that their experience showed that the international campaign strategy brought local unions to focus more on reaching international donor project's goals than unionization.

\subsection{Global/short- and long-term: bi-directional partnership and mutual understanding}

The local mobilization and organizing is then built upon to foster global collaboration and ultimately transnational solidarity. It has been argued that many transnational initiatives suffer from a North-South bias and that transnational solidarity is often 'one-sided' (e.g. Featherstone, 2012), with existing power asymmetries being overlooked or even reproduced. Transnational solidarity however builds on mutuality despite differences (Hyman, 2011), thus, the forging of a collective identity (Davis, 2017), while at the same time acknowledging differences of workers in different contexts. ExChains actively seeks to avoid such biases, following an approach of 'transnational partnership' which, first, seeks to address workers in the periphery as change agents and, second, aims to foster a support network that is explicitly seen as bi-directional. As stated by the network itself:

"ExChains wants to help irritate the Northern perspective on women and men in the global South as mere victims of their social conditions. Working closely with unions and workers in the global South, the campaign takes them seriously as actors. Actions are being jointly planned and coordinated." (ExChains, 2012)

However, such feelings of transnational partnership rather than one-sided aid have to be actively created and constantly maintained. While the initial motivation to engage in the ExChains network - especially by workers form the global North-is often a feeling of discontent with the situation in the Global South and an accordant motivation to "help", the ExChains network seeks to develop this feeling of 'wanting to help' into one of 'partnership' and 'mutual support' as one of our interviewees reports (180309). He goes on to argue that while the motivation to help is certainly important to mobilize workers, it is important to the members of the ExChains network to not see such motivation as an end-point, but rather ask how it might open up possibilities to support each other, in order to avoid trapping into paternalistic practices. He thus underlines: "It is a big part of our political work to discuss at length what we have in common and why we work together-beyond the motivation to say 'I perceive the current situation as unfair"' (180309). Also on the side of workers from garment production, the approach of 'mutual support' requires a change in perspective. He recounts, for instance the realization that support from their side can be meaningful and can have an impact for workers from garment retail. "Here in Bangladesh we will also support the struggles and demands of German retail workers with campaigns. That's clear to us" (worker from NGWF cited in ExChains, 2016). Such processes of understanding and realization are enabled during Skype conferences and mutual visits, such as a visit from works council members from H\&M and Zara as well as members of the TIE network in India and Sri Lanka in 2014, but also need to be part of the everyday discussions at both sides of the supply chain. Given the undeniable 
structural differences between workers in garment retail and production, he also adds that for everyone involved it remains a constant struggle to challenge one's own prejudices and perceptions: "So, I do not think that we are immune or protected from it (stereotyped perceptions). But it's part of our political work that we try to break out of them" (180309).

Given that even on the local, national level the existence of a shared 'worker identity' as well as shared interests among workers-as one ingredient to solidarity-cannot be assumed and, as we have seen above, needs to be created, the creation of a shared consciousness and the carving out of shared interests appears even more difficult on the transnational level, where huge - geographical and cultural—distances as well as enormous economic differences between workers exist. Despite all these differences and without talking them down, ExChains seeks to actively create shared perceptions and interests. This requires the creation of a mutual understanding between workers along the supply chain:

"The discussion of common ground and differences is central to our work, because the work in the ExChains network is based on the fact that employees at all places along the production chain meet as colleagues and work together on equal terms. This requires understanding for the situation of each other" (ExChains, 2015).

During visits for instance, the above mentioned workplace and health mappings are also used to identify the similarities and differences between the situation of workers in garment retail and production. One of our interviewees gives an account of such a mapping and its effect on the workers' perception:

"We just started by comparing what amount of their monthly pay participants spend for certain things, where commonalities and differences lie and what that means etc. And that causes some irritation: on the one hand it irritates the image of our colleagues in Asia that people from the North don't have any problems. And on the other hand, it irritates our image to say that in Asia everything is doomed and we only have luxury problems" (180903).

Although working and living conditions of workers in garment retail and production are different in many ways, some commonalities can be identified and are put into focus by the members of the ExChains network: for instance the experiences of humiliation, annoyance at indecent conditions, or difficulties concerning union and workplace organising-despite variance in their degree-are highlighted as shared issues between workers along the supply chain (ExChains, 2011). What is also emphasized during these visits and exchanges is that next to a feeling of shared experiences, these experiences are caused by the same actors:

\footnotetext{
"You can imagine it like this: The active workers came together and started to tell each other about themselves and then something become clear: the problems in retail in Germany are comparable to the problems we find in India and Bangladesh — on a different level for sure, but generally speaking they are comparable. And at some point, we came to the conclusion that this was of course not surprising, because the actors are the same everywhere - be it here as an employer or there in India as a buyer" (170922).
}

The creation of shared identity and interests along the supply chain, thus, can be also fostered by emphasizing the shared opponent. Emphasis is thus put on the fact that the powerful actors responsible for the working conditions of both retail and production workers are the same: lead firms. Workers in the periphery-both in retail and production-thus address the same actors in their fights. This creates a further reason for why collective action might_-despite all differences_-be important. 
"Companies make profits at the expense of employees. They reduce wages, increase working hours and demand more and more from the workers; here in Germany and even more so in Bangladesh. (...) We can only fight back together" (worker from NGWF cited in ExChains, 2013).

Through such linkages, local fights in garment production can be supported by garment retail worker's involvement of lead firms. For instance, the workers in the supplier factories, together with their unions, are developing demands and ways to negotiate them with factory owners on site. These can be demands for drinking water or clean toilets, but also more extensive demands for higher wages or shorter working hours. In their actions and negotiations, the workers include the lead firms that produce in their factories, which can effectively be supported by workers from garment retail.

"Some of the demands we can enforce ourselves, for others we will have to go beyond the factory level and bring lead firms to the negotiating table. They determine the conditions most under which we work" (worker from GATWU cited in ExChains, 2016).

Through such active framing processes mutual understanding is created. Telling each other about their work and private lives, a feeling of shared experiences is created among workers, because it becomes apparent that at least some of their problems are shared by their coworkers as well as by workers in other parts of the supply chain. Workers also learn that the hardships they face are often caused by the same source, namely buyers' demands. This facilitates an understanding of why it makes sense to engage in collective and mutual support and provides the ground for the formulation of shared interests and demands. Understanding the problems of others can also support the realization of ones' own situation and the creation of individual agency: "I recognize myself through the other" as one of our interviewees put it (180309). Here, the exchange on the transnational level feeds back and reinforces the work on the local level.

Besides ExChains' ability to balance the tensions revolving around transnational solidarity, a few more structural characteristics make ExChains' different from other initiatives. First, compared to other cross-border networks, ExChains brings together workers from different sectors - garment production and garment retail-thereby addressing workers that do not stand in direct competition to one another. Second, as there is almost no labour mobility between Asian production countries and European retail countries, workers do not compete for jobs. Whereas in "clothing manufacturing, unions in the global North have not been able to prevent the exodus of jobs to the global South" (Anner et al., 2004: 8), retail cannot be relocated and thus the retail sector in the North and production in the South do not stand in competition with one another. In such a constellation, employers cannot play off groups of workers against each other.

\section{Discussion}

Given the frequent labour standards violations in the garment industry, it is paramount to find ways of strengthening the bargaining power of workers along the supply chain. Departing from a critical review of the previous literature on transnational union coordination and collaboration as mixing the structural, associational, institutional, societal, and ideational components of transnational collaboration and coordination, we highlighted the challenges 
of understanding transnational solidarity as a cultural construction in a globalized social space. Particularly, we highlighted that the concept of solidarity adds an understanding of the cultural-normative dimension of unions' collective action that differs from their structural, associational, institutional, and societal power, and hence may be considered as part of an independent ideational power resource itself. Using a case study on an initiative meant to foster transnational solidarity among garment and retail workers connected in a buyer-driven global supply chain, we have argued that structural, institutional, associational, and societal power-though to varying degrees - are weak in both garment-producing and garment-selling countries. We have furthermore argued that previous forms of transnational initiatives - most importantly international campaigns, GFAs, and accords-often fail to establish mutual support among workers and are-in their current forms-better understood as some sort of aid than as forms of transnational solidarity.

Given these challenges of previous initiatives, it is important to understand the structures and mechanisms by which particularly the associational power of workers in global supply chains can be strengthened and how transnational workers solidarity-understood as mutual bonds among groups of workers - can be established. By analyzing ExChains' attempts to mobilize and organize workers for transnational solidarity, we see that transnational solidarity depends on an active facilitation of interactive processes among workers that create shared experiences and a common perception that norms have been violated. Skype conferences and mutual site visits are used for exercises to reflect on one's own problems and identify commonalities and differences between workers in garment production and retail. We have furthermore highlighted the importance of local worker mobilization and organization based on direct links between workers and local unions as a supplement to official relationships between global union federations and international organisations, and the accordant importance of bridging short-term gains with long-term goals for establishing sustainable forms of transnational labour solidarity. What ExChains thus shows us it that the importance of local mobilizing and organizing lies in the process. Realizing short-term goals for workers develops a sense of empowerment and builds commitment. The realization of these local and short-term goals then allows to connect globally and approach longer-term goals. It also shows that fostering ideational power through digital technology (Heckscher \& McCarthy, 2014) as well as through more conventional means such as sharing health mapping documents or organizing mutual visits of workers across the supply chain might be a useful complement to extant discussions of transnational labour power (Zajak, 2017).

We have argued that this ExChain's strategy goes beyond what previous transnational initiatives achieve. Its potential lies in the fact that the analysis of problems and the formulation of demands for changing the conditions at the workplace arise directly in a collective process among workers at the factory and shop level. This is important as "transnational solidarity will have strong leverage only when shared by rank and file workers" (Lindberg, 2011, p. 206). Such a strategy perceives workers in garment factories as active subjects rather than passive receivers of international aid, information providers or 'watch dogs' over standard compliance for international actors. Workers are thus not seen within "'categories of need' but are considered active citizens with strength and talent, democratic and entrepreneurial potential" (Tapia, 2013, p. 675). This shift in perspective provides the ground for mobilizing and organizing workers and accounts for the often proclaimed idea that sustain- 
able transnational solidarity needs to be organized, but also results from organizing (e.g. Davis, 2017). Focusing on local organizing in this way therefore bears the potential to foster sustained labour power rather than build on temporary or 'borrowed' power of international actors. That this strategy promises success is confirmed by victories such as in Bangalore, where through joint action and negotiation among workers from garment production and retail the lay-off of cleaners could be hindered and three free Sundays per month could be negotiated for workers in a local factory (ExChains, 2016).

Although we are confident that our case highlights how the formation of solidarity can be supported through active efforts to organize for the emergence of weak ties bottom-up in transnational space even where strong associational ties or class identities do not exist, we must concede that it is too early to say whether ExChains' efforts will have a lasting impact. Given the sheer number of garment workers in countries like India or Bangladesh as well as the huge structural difficulties reported above, efforts like the one of the ExChains' initiative certainly remain limited, particularly conerning the endurance and reproducibility of the efforts. What happens if the single activists involved from all sides change? How are conflicts resolved if divergence of goals materializes in contradictory demands? And how are gains defended against oppositions from single employers? One way to deal with these and similar challenges is to connect to already existing global networks such as global unions federations in order to translate the ideational solidarities into institutional, associational, and societal power resources.

Bringing the locally organized workers together is important to withstand powerful employers on the transnational level and acknowledges that those employers are often the same for workers in garment retail and production. An approach of constructing transnational workers solidarity by trust-based, relational culture-based forms mobilization that create member commitment instead of focusing directly on organizing campaigns (Tapia, 2013) along the supply chain thus responds to the fact that the global and the local are intrinsically tied in supply chains. While oppression is produced at all scales, workers must organize simultaneously across space as well as in space (Davis, 2017). Local actors need to 'jump scale' (Merk, 2009), but transnational organization needs to build on effective local structures. That is, while counter-movements need to be global (Burawoy, 2010), the local cannot be dismissed (Caspersz, 2010).

In sum, our study not only reiterates that new forms of associational power are needed to organize precarious workers (see Fichter et al., 2018), but argues that a stronger focus on ideational power resources, particularly the creation of solidarity relations among workers, might be a fruitful starting point for organizing countervailing worker power. Further research might investigate how the relationship between such grassroots organizing and unions' more higher-level organizing develops over time. Do these activities unfold largely in parallel? Does one undermine the operational efficiency of the other? Or do they mutually support each other? Additionally, further research should look for similar initiatives in other sectors. Are the strategies for organizing transnational solidarity among workers similar or different in buyer- versus producer-driven supply chains, for instance? Finally, our conceptualization of ideational power and particularly its foundations-solidarity, but also shared knowledge, traditions or customs — should be examined further. 


\section{References}

Alexander, R., Ashwin, S., Lohmeyer, N., Oka, C. \& Schüßler, E. (2017). Analysing the Evolving Texture of Transnational Industrial Relations: Opening the Black Box of Interfirm and FirmUnion Relationships in the Global Garment Industry. Garment Supply Chain Governance Discussion Paper Series No. 01/2017.

Anderson, J. (2015). Towards resonant places: reflections on the organizing strategy of the International Transport Workers' Federation. Space and Polity, 19, 47-61. doi: 10.1080/13562576.2014.991118

Anner, M. (2015). Labour control regimes and worker resistance in global supply chains. Labour History, 56 (3), 292-307. doi: 10.1080/0023656X.2015.1042771

Anner, M. (2009). Two Logics of Labor Organizing in the Global Apparel Industry. International Studies Quarterly, 53, 545-570. doi: 10.1111/j.1468-2478.2009.00546.x

Anner, M. (2000). Local and Transnational Campaigns to End Sweatshop Practices. In M. Gordon \& L. Turner (eds), Transnational Cooperation among Labour Unions (pp. 238-55). Ithaca, NY: ILR Press.

Anner, M., Greer, I., Hauptmeier, M., Lillie, N. \& Winchester, N. (2004). The Industrial Determinants of Transnational Solidarity: Global Interunion Politics in Three Sectors. European Journal of Industrial Relations, 12 (1), 7-27. doi: 10.1177/0959680106061368

Appelbaum, E. \& Schmitt, J. (2009). Low-wage work in high-income countries: Labor-market institutions and business strategy in the US and Europe. Human Relations, 62 (12), 1907-1934. doi: $10.1177 / 0018726709349200$

Banting, K. G. \& Kymlicka, W. (2017). The Strains of Commitment: The Political Sources of Solidarity in Diverse Societies. Oxford Scholarship Online. doi: 10.1093/acprof:oso/9780198795452.001.0001

Barrientos, S. \& Smith, S. (2007). Do workers benefit from ethical trade? Assessing codes of labour practice in global production systems. Third World Quarterly, 28 (4), 713-729. doi: 10.1080/01436590701336580

Baumann-Pauly, D., Labowitz, S. \& Banerjee, N. (2015). Closing Governance Gaps in Bangladesh's Garment Industry - The Power and Limitations of Private Governance Schemes (March 12, 2015). Available at SSRN: https://ssrn.com/abstract=2577535 or doi: $10.2139 /$ ssrn. 2577535.

Bieler, A. (2012). 'Workers of the world, unite'? Globalisation and the Quest for Transnational Solidarity. Globalizations, 9 (3), 365-378. doi: 10.1080/14747731.2012.680730

Bieler, A. \& Erne, R. (2014). Transnational Solidarity? The European Working Class in the Eurozone Crisis. Socialist Register, 51, 157-177.

Bieler, A., Erne, R., Golden, D., Helle, I., Kjelstadli, K., Matos, T. M. \& Stan, S. (eds.) (2015). Labour and Transnational Action in Times of Crisis. London: Rowman \& Littlefield.

Bieler, A. \& Lindberg, I. (eds.) (2011). Global restructuring, labour and the challenges for transnational solidarity. New York: Routledge.

Bieler, A., Lindberg, I. \& Pillay, D. (2008). Labour and the challenges of globalization: what prospects for transnational solidarity? London, Ann Arbor/MI: Pluto Press.

Bormann, S., Jungülsing, J., Bian, S., Hartung, M. \& Schubert, F. (eds.). (2015). Last Call for Solidarity. Perspektiven grenzüberschreitenden Handelns von Gewerkschaften. Hamburg: VSA Verlag.

Bosch, G. \& Lehndorff, S. (eds.) (2004). Working in the service sector: A tale from different worlds. London, New York: Routledge. doi: 10.4324/9780203500583

Brinkmann, U., Choi, H. L., Detje, R., Dörre, K., Holst, H., Karakayali, S. \& Schmalstieg, C. (2008). Strategic Unionism: Aus der Krise zur Erneuerung? Wiesbaden: VS Verlag für Sozialwissenschaften.

Burawoy, M. (2010). From Polanyi to Pollyanna: The false optimism of global labour studies. Global Labour Journal, 1 (2), 301-313. doi: 10.15173/glj.v1i2.1079 
Brookes, M. (2013). Varieties of power in transnational labour alliances: An analysis of workers' structural, institutional and coalitional power in the global economy. Labour Studies Journal, 38 (3), 181-200. doi: 10.1177/0160449X13500147

Caspersz, D. (2010). From Pollyanna to the Pollyanna Principle. A Response to Michael Burawoy's 'From Polanyi to Pollyanna: The False Optimism of Global Labour Studies'. Global Labour Journal, 1 (3), 393-397. doi: 10.15173/glj.v1i3.1087

Croucher, R. \& Cotton, E. (2009). Global unions, global business. Global union federations and international business. Middlesex University Press: London.

Crozier, M. \& Friedberg, E. (1979). Macht und Organisation. Die Zwänge kollektiven Handelns. Königstein: Athenäum-Verlag.

Davis, S. (2017). Sharing the struggle: constructing transnational solidarity in global social movements. Space and Polity, 21 (2), 158-172. doi: 10.1080/13562576.2017.1324255

Doellgast, V., Lillie, N. \& Pulignano, V. (2018). From dualization to solidarity. Halting the cycle of precarity. In V. Doellgast, N. Lillie \& V. Pulignano (eds.), Reconstructing solidarity. Labour unions, precarious work, and the politics of institutional change in Europe (pp. 1-43). Oxford University Press: Oxford. doi: 10.1093/oso/9780198791843.003.0001

Dörre, K., Holst, K. \& Nachtwey, O. (2009). Organizing: A strategic option for trade union renewal? International Journal of Action Research, 5 (1), 33-67.

Donaghey, J., Reinecke, J., Niforou, C. \& Lawson, B. (2014). From employment relations to consumption relations: Balancing labor governance in global supply chains. Human Resource Management, 53 (2), 229-252. doi: 10.1002/hrm.21552

Dribbusch, H. (2014). Voraussetzungen internationaler Solidarität: Zur Diskussion um einen europäischen Generalstreik, WSI-Mitteilungen, 5/2014, 337-344. doi: 10.5771/0342-300X-2014-5-337

ExChains (2016). Beschäftigte entlang der globalen Zulieferkette: Stark durch neue Strategie!, ExChains Nachrichten 06/2016.

ExChains (2015). Gemeinsamer Widerstand entlang der globalen Zulieferkette, ExChains Nachrichten $05 / 2015$.

ExChains (2013). Nur gemeinsam können wir wirklich etwas verändern, ExChains Nachrichten 08/2013.

ExChains (2012). Joint Campaign for Workers' Rights, ExChains Campaign Flyer, 2012.

ExChains (2011). Workers' exchange between Germany and Bangladesh, ExChains Newsletter, 14/2011.

Fairbrother, P. \& Hammer, N. (2005). Global unions: Past efforts and future prospects. Relations Industrielles/Industrial Relations, 60 (3), 405-431. doi: 10.7202/012153ar

Fantasia, R. (1995). From class consciousness to culture, action, and social organization. Annual Review of Sociology, 21 (1), 269-287. doi: 10.1146/annurev.so.21.080195.001413

Fantasia, R. (1988). Cultures of solidarity. Consciousness, action, and contemporary American workers. Berkeley: University of California Press.

Featherstone, D. (2012). Solidarity: Hidden histories and geographies of internationalism. London, New York: Zed Books Ltd.

Fichter, M., Ludwig, C., Schmalz, S., Schulz, B. \& Steinfeldt, H. (2018). The transformation of organised labour. Mobilising power resources to confront $21^{\text {st }}$ century capitalism. Friedrich-EbertStiftung: Berlin.

Frege, C. \& Kelly, J. E. (eds.). (2004). Varieties of unionism: Strategies for union revitalization in a globalizing economy. Oxford: Oxford University Press.

doi: 10.1093/acprof:oso/9780199270149.001.0001

Gennard J. \& Newsome, K. (2005). Barriers to cross border trade union cooperation in Europe: the case of the graphical workers. Industrial Relations Journal, 36 (1), 38-58.

doi: $10.1111 /$ j.1468-2338.2005.00344.x 
Geppert, M. \& Pastuh, J. (2017). Total institutions revisited: What can Goffman's approach tell us about 'oppressive' control and 'problematic' conditions of work and employment in contemporary business organizations? Competition \& Change, 21 (4).

doi: $10.1177 / 1024529417722350$.

Geppert, M., Williams, K. \& Wortmann, M. (2015). Micro-political game playing in Lidl: A comparison of store-level employment relations. European Journal of Industrial Relations, 21 (3), 241 257. doi: $10.1177 / 0959680114544015$

Gereffi, G. (1994). The organization of buyer-driven global commodity chains: How US retailers shape overseas production networks. In G. Gereffi \& M. Korzeniewicz (eds), Commodity Chains and Global Capitalism (pp. 95-122). Westport, CT: Praeger.

Gordon, M. E. \& Turner, L. (eds.) (2000). Transnational cooperation among labor unions. Ithaca: ILR press.

Grannies, R., Smith, D.A. \& Stepan-Norris, J. (2008). Working connections: Shop floor networks and union leadership. Sociological Perspectives, 51 (3), 649-672. doi: 10.1525/sop.2008.51.3.649

Greer, I., Ciupijus, Z. \& Lillie, N. (2013). The European Migrant Workers Union and the barriers to transnational industrial citizenship. European Journal of Industrial Relations, 19 (1), 5-20. doi: 10.1177/0959680112474748

Grimshaw, D., Marchington, M., Rubery, J. \& Willmott, H. (eds.). (2005). Fragmenting work: Blurring organizational boundaries and disordering hierarchies. Oxford: Oxford University Press.

Grugulis, I. \& Bozkurt, O. (2011). Retail work. Basingstoke: Palgrave Macmillan. doi: $10.1007 / 978-0-230-34488-4$

Hardy, J. (2015). Explaining 'varieties of solidarity': labour mobility and trade unions in an enlarged Europe. Transfer: European Review of Labour and Research, 21 (2), 187-200. doi: $10.1177 / 1024258915573186$

Hardy, J., Calveley, M., Kubisa, J. \& Shelley, S. (2015). Labour strategies, cross-border solidarity and the mobility of health workers: Evidence from five New Member States. European Journal of Industrial Relations, 21 (4), 315-333. doi: 10.1177/0959680114553159

Heckscher, C. \& McCarthy, J. (2014). Transient Solidarities: Commitment and Collective Action in Post-Industrial Societies. British Journal of Industrial Relations, 52 (4), 627-657. doi: 10.1111/bjir.12084

Helfen, M. \& Fichter, M. (2013). Building transnational union networks across global production networks: Conceptualising a new arena of labour-management relations. British Journal of Industrial Relations, 51 (3), 553-576. doi: 10.1111/bjir.12016

Helfen, M., Schüßler, E. \& Sydow, J. (2018). How can employment relations in global value networks be managed towards social responsibility? Human Relations, in print. doi: $10.1177 / 0018726718757060$

Helfen, M. O., Schüßler, E. \& Stevis, D. (2016). Translating European labor relations practices to the United States through global framework agreements? German and Swedish multinationals compared. ILR Review, 69 (3), 631-655. doi: 10.1177/0019793915624090

Herod, A. (2000). Implications of Just-in-Time Production for Union Strategy: Lessons from the 1998 General Motors-United Auto Workers Dispute. Annals of the Association of American Geographers, 90 (3), 521-547.

Hyman, R. (2011). Gewerkschaftliche Strategien und Solidaritätspolitik unter globalen Konkurrenzbedingungen. In F. Gerlach, Th. Greven, U. Mückenberger \& E. Schmidt (eds.), Solidarität über Grenzen. Gewerkschaften vor neuer Standortkonkurrenz (pp. 51-72). Berlin: edition sigma. doi: $10.5771 / 9783845269474-51$

Hyman, R. (2005). Shifting dynamics in international trade unionism. Agitation, organization, bureaucracy, diplomacy. Labor History, 46 (2), 137-154. doi: 10.1080/00236560500080758 
Hyman, R. (2001). Understanding European trade unionism: between market, class and society. London: Sage.

Jessup, D. \& Gordon, M. E. (2000). Organizing in export processing zones: The Bibong Experience in the Dominican Republic. Cornell International Industrial and Labor Relations Reports, 36, 179-201.

Juravich, T. (2007). Beating global capital. A framework and method for union strategic corporate research and campaigns. In K. Bronfenbrenner (ed.), Global unions. Challenging transational capital through cross-border campaigns (pp. 16-39). Ithaca: ILR Press.

Jürgens, U. (1984). Die Entwicklung von Macht, Herrschaft und Kontrolle im Betrieb als politischer Prozeß - Eine Problemskizze zur Arbeitspolitik. In U. Jürgens \& F. Naschold (eds.), Arbeitspolitik. Materialien zum Zusammenhang von Macht, Kontrolle und betrieblicher Organisation der Arbeit (pp. 58-91). Opladen: Westdeutscher Verlag. doi: 10.1007/978-3-322-89388-8_2

Kabeer, N. \& Mahmud, S. (2018). Multi-stakeholder initiatives in Bangladesh after Rana Plaza: global norms and workers' perspectives. Paper presented at the workshop "5 Years After Rana Plaza: Consequences for Labor Standards Improvements in Garment Supply Chains", Berlin, April 27-28, 2018.

Köhnen, H. (2006). Unternehmenskultur und Personalpolitik. Zur Situation der Beschäftigten und der Interessenvertretung bei H\&M. Arbeitspapier 119. Düsseldorf: Hans-Böckler-Stiftung.

Kotthoff, H. \& Whittall, M. (2014). Paths to transnational solidarity: identity building in European works councils. Oxford: Peter Lang.

Labowitz, S. \& Baumann-Pauly, D. (2015). Beyond the tip of the iceberg: Bangladesh's forgotten apparel workers. NYU Stern Center for Business and Human Rights. Online:

http://people.stern.nyu.edu/twadhwa/bangladesh/downloads/beyond_the_tip_of_the_iceberg_rep ort.pdf.

Levesque, C. \& Murray, G. (2010). Trade union cross-border alliances within MNCs: disentangling union dynamics at the local, national and international levels. Industrial Relations Journal, 41 (4), 312-332. doi: 10.1111/j.1468-2338.2010.00571.x

Levy, D. L. (2008). Political contestation in global production networks. Academy of Management Review, 33 (4), 943-963. doi: 10.5465/amr.2008.34422006

Lindberg. I. (2011). Varieties of solidarity: an analysis of cases of worker action across borders. In A. Bieler, \& O. Lindberg (eds.), Global Restructuring, Labour and the Challenges for Transnational Solidarity (pp. 206-219). London: Routledge.

Locke, R., Amengual, M. \& Mangla, A. (2009). Virtue out of necessity? Compliance, commitment, and the improvement of labor conditions in global supply chains. Politics \& Society, 37 (3), 319351. doi: 10.1177/0032329209338922

Lund-Thomsen, P. \& Lindgreen, A. (2014). Corporate social responsibility in global value chains: Where are we now and where are we going?. Journal of Business Ethics, 123 (1), 11-22. doi: 10.1007/s10551-013-1796-x

Luterbacher, U., Prosser, A. \& Papadakis, K. (2017). An emerging transnational industrial relations? Exploring the prospects for cross-border labour bargaining. International Labour Review, 156 (3-4), 307-343. doi: 10.1111/ilr.12078

Merk, J. (2010). Cross-border wage struggles in the global garment industry: the campaign for an Asia floor wage. In A. Bieler \& J. W. Goudriaan (eds.), Global Restructuring, Labour and the Challenges for Transnational Solidarity (pp. 116-130). London: Routledge.

Merk, J. (2009). Jumping scale and bridging space in the era of corporate social responsibility: Crossborder labour struggles in the global garment industry. Third World Quarterly, 30 (3), 599-615. doi: 10.1080/01436590902742354 
Mosley, L. (2017). Workers' rights in global value chains: possibilities for protection and for peril. New Political Economy, 22 (2), 153-168. doi: 10.1080/13563467.2016.1273339

Müller, T., Platzer, H.-W. \& Rüb, S. (2006). Einführung: Globalisierung und transnationale Arbeitsbeziehungen. Industrielle Beziehungen: Zeitschrift für Arbeit, Organisation und Management, 13 (3), 197-204. doi: 10.1007/978-3-322-81475-3_1

Offe, C. \& Wiesenthal, H. (1980). Two Logics of Collective Action. Theoretical Notes on Social Class and Organizational Form. Political Power and Social Theory, 1, 67-115.

Oka, C. (2016). Improving working conditions in garment supply chains: The role of unions in Cambodia. British Journal of Industrial Relations, 54 (3), 647-672. doi: 10.1111/bjir.12118

Palpacuer, F. (2008). Bringing the social context back in: governance and wealth distribution in global commodity chains. Economy and Society, 37 (3), 393-419. doi: 10.1080/03085140802172698

Papadakis, K. (ed.). (2008). Cross-border Social Dialogue and Agreements. An emerging global industrial relations framework?. International Labor Office.

Pernicka, S., Glassner, V., Dittmar, N., Mrozowicki, A. \& Maciejewska, M. (2017). When does solidarity end? Transnational labour cooperation during and after the crisis-the GM/Opel case revisited. Economic and Industrial Democracy, 38 (3), 375-399. doi: 10.1177/0143831X15577840

Pernicka, S. \& Glassner, V. (2014). Transnational trade union strategies towards European wage policy: A neo-institutional framework. European Journal of Industrial Relations, 20 (4), 317-334. doi: $10.1177 / 0959680113518232$

Platzer, H. W. \& Müller, T. (2009). Die globalen und europäischen Gewerkschaftsverbände: Handbuch und Analysen zur transnationalen Gewerkschaftspolitik. Edition Sigma. doi: $10.5771 / 9783845269337$

Pries, L. \& Seeliger, M. (2013). Work and employment relations in a globalized world: The emerging texture of transnational labour regulation. Global Labour Journal, 4 (1), 26-47. doi: $10.15173 / g l j . v 4 i 1.1127$

Reinecke, J. \& Donaghey, J. (2015). After Rana Plaza: Building coalitional power for labour rights between unions and (consumption-based) social movement organisations. Organization, 22 (5), 720-740. doi: 10.1177/1350508415585028

Sassen, S. (2012). Interactions of the technical and the social. Digital formations of the powerful and the powerless. Information, Communication \& Society, 15 (4), 455-478. doi: 10.1080/1369118X.2012.667912

Simms, M. \& Dean, D. (2015). Mobilising contingent workers: An analysis of two successful cases. Economic and Industrial Democracy, 36 (1), 173-190. doi: org/10.1177/0143831X13501000

Schmalz, S. \& Dörre, K. (2014). Der Machtressourcenansatz: Ein Instrument zur Analyse gewerkschaftlichen Handlungsvermögens. Industrielle Beziehungen/The German Journal of Industrial Relations, 21 (3), 217-237.

Schüßler, E., Frenkel, S. \& Wright, C. W. (2018). Governance of Labor Standards in Australian and German Garment Supply Chains: The Impact of Rana Plaza. ILR Review. In print. doi: 10.1177/0019793918771360

Silver, B. J. (2003). Forces of Labour: Workers' Movements and Globalization since 1870. New York: Cambridge University Press. doi: 10.1017/CBO9780511615702

Stevis, D. \& Boswell, T. (2007). Globalization and Labor: Democratizing Global Governance. Lanham: Rowman\&Littlefield.

Tapia, M. (2013). Marching to different tunes: Commitment and culture as mobilizing mechanisms of trade unions and community organizations. British Journal of Industrial Relations, 51 (4), 666688. doi: 10.1111/j.1467-8543.2012.00893.x

Wirth, C. (2016). Modularer Einzelhandel und industrielle Beziehungen. Ergebnisse einer qualitativen Längsschnittuntersuchung, 1991-2012. Münster: Westfälisches Dampfboot. 
Wright, E. O. (2000). Working-class power, capitalist-class interests, and class compromise. American Journal of Sociology, 105 (4), 957-1002. doi: 10.1086/210397

Zajak, S. (2017). International allies, institutional layering and power in the making of labour in Bangladesh. Development and Change, 48 (5), 1007-1030. doi: 10.1111/dech.12327 


\section{Martin Seeliger}

\section{Die soziale Konstruktion internationaler Solidarität. Gewerkschaftspolitische Positionsbildung im Bereich der Dienstleistungsfreiheit ${ }^{\star *}$}

\section{Zusammenfassung}

Aus gewerkschaftlicher Sicht findet Zusammenarbeit im grenzüberschreitenden Rahmen von jeher unter dem Aspekt ,internationaler Solidarität' statt. Am Beispiel der gewerkschaftlichen Positionsbildung im Bereich der Dienstleistungsfreiheit untersucht der Artikel ihr Zustandekommen als einen sozialen Konstruktionsprozess. Der Fokus auf Organisationen aus Schweden, Polen und Ungarn sowie ihre Zusammenarbeit im Rahmen des Europäischen Gewerkschaftsbundes berücksichtigt mit der politökonomischen Heterogenität der Union die wichtigste Herausforderung gewerkschaftlicher Kooperation innerhalb der EU. Vor diesem Hintergrund gelangt der Artikel zu drei Befunden: Erstens stellt internationale Solidarität das Resultat mitunter konfliktiver Aushandlung über Möglichkeit und Notwendigkeit dar. Um Solidarität zu generieren, ist es notwendig, einen Deutungsrahmen (,Soziales Europa') zu etablieren. Als Resultat eines Aushandlungsprozesses kommt diesem Deutungsrahmen zweitens ein genuin politischer Charakter zu. Hier übertragen sich die bessere Ressourcenausstattung und der Etablierten-Status der westeuropäischen Vertreter in eine politische Dominanz gegenüber den Repräsentanten der neuen Mitgliedsländer. Eine deliberative Auseinandersetzung auf Augenhöhe erscheint vor diesem Hintergrund derzeit zwar als wünschenswert, jedoch unrealistisch.

Schlagwörter: Internationale Solidarität, Gewerkschaftspolitik, Dienstleistungsrichtlinie, Soziales Europa, Framing

The social construction of international solidarity. Trade union politics in the field of the freedom of services

\section{Abstract}

From a trade union perspective, cooperation in a cross-border context is often filed under the term 'international solidarity'. Drawing on the example of the trade union position on the freedom of services, this article considers solidarity as a process of social construction. Focusing on cooperation between

* Dr. Martin Seeliger, Europa-Universität Flensburg, Seminar für Soziologie, Auf dem Campus 1, 24943 Flensburg. E-Mail: martin.seeliger@gmx.net

** Artikel eingegangen: 26.3.2018, revidierte Fassung akzeptiert nach doppelt-blindem Begutachtungsverfahren: 10.09.2018 
organizations from Sweden, Poland and Hungary within the framework of the European trade union federation we consider how socioeconomic heterogeneity within the EU-28 generates challenges for trade union cooperation. Against this background, the article produces three findings: Firstly, international solidarity is the result of (conflictual) negotiations over its possibility and necessity. Hence, in order to generate solidarity, trade unions need to establish a meaningful framework of what a "Social Europe" represents. Secondly, as a consequence of such negotiations this framework possesses a genuinely political character. However, better access to resources and as well as having the benefit of an established status results in a dominance of Western European representatives. Thirdly, cooperation on a level playing field may currently appear desirable, but as of yet quite unrealistic.

Keywords: International solidarity, Trade union politics, freedom of services, Bolkestein Directive European Trade Union Confederation (JEL: O52, L8, B5)

\section{Einleitung}

„Wer im Stich lässt seines Gleichen, lässt ja nur sich selbst im Stich“, heißt es im bekannten ,Solidaritätslied' von Berthold Brecht und Hanns Eisler. ${ }^{1}$ Ganz in diesem Sinne stellt das Funktionsprinzip von Lohnabhängigensolidarität von jeher einen zentralen politischen Bezugsrahmen gewerkschaftspolitischen Handelns dar.

Ein wesentliches Problem, welches sich in der Praxis hieraus ergibt, behandeln die Urheber des bekannten Liedes im Text allerdings nicht. Konkret folgt es aus den Fragen, wer denn genau ,seines Gleichen“ sein soll, und unter welchen Umständen man diesen ,im Stich' ließe, oder - mit anderen Worten - wer Mitglied der Solidargemeinschaft ist und was der Gegenstand des solidarischen Handelns sein soll. Die Aushandlung dieser Angelegenheiten in der politischen Praxis wollen wir im Folgenden als soziale Konstruktion von Solidarität bezeichnen.

Die zunehmende Intensität, mit der diese Frage unter den Gewerkschaften der EU in den letzten Jahren diskutiert worden ist, ergibt sich aus den wirtschaftlichen Konsequenzen der europäischen Integration. Während die qualitative Vertiefung des gemeinsamen Marktes den Wettbewerb zwischen europäischen Lohnabhängigen intensiviert hat, machen es die zunehmenden Unterschiede zwischen den Mitgliedsländern den Gewerkschaften schwer, diesem Umstand mit einer gemeinsamen politischen Linie zu begegnen. Der Frage, inwiefern sich diese Entwicklungen innerhalb der europäischen Gewerkschaftsbewegung in eine solidarische Form der Zusammenarbeit übertragen, geht der vorliegende Artikel anhand der Diskussion um die Dienstleistungsfreiheit als zentralem Feld europäischer Tarifpolitik nach. Vor dem Hintergrund der EU-Osterweiterung war es hier gelungen, mit Hilfe einer von ost- und westeuropäischen Gewerkschaften mitgetragenen Kampagne eine gesetzliche Regelung zu verhindern, welche es osteuropäischen Lohnabhängigen (und damit auch dem westeuropäischen Kapital) ermöglicht hätte, die Beschäftigungsstandards der alten Mitgliedsländer auf deren Territorium zu unterschreiten. Das Zustandekommen dieser gemeinsamen Position untersucht der vorliegende Artikel unter dem Blickpunkt der sozialen Konstruktion internationaler Solidarität.

1 Für wertvolle Kommentare bedanke ich mich bei Floris Biskamp, Martin Höpner und Moritz Müller. 
Ein besonderer Fokus auf die Beteiligung von Gewerkschaften aus Polen, Ungarn und Schweden im Rahmen der Auseinandersetzung im Europäischen Gewerkschaftsbund dient zur Abbildung der politökonomischen Heterogenität der Union. Auf dieser Grundlage stehen am Ende der Darstellung drei weiterführende Forschungsbefunde zur sozialen Konstruktion von Solidarität im Rahmen internationaler Gewerkschaftspolitik:

1) Die handlungstheoretische Rekonstruktion der Diskussionen um die Dienstleistungsrichtlinie zeigt, wie solidarische Haltungen politischer Akteure als Resultat mitunter konfliktiver Aushandlung über Möglichkeit und Notwendigkeit von Solidarität entstehen. Um Solidarität zu generieren, ist es notwendig, einen Deutungsrahmen (,Soziales Europa') zu etablieren.

2) Als Resultat eines Aushandlungsprozesses kommt diesem Deutungsrahmen (nicht nur im Ergebnis, sondern auch im Zustandekommen) ein genuin politischer Charakter zu. Unter welchen Bedingungen gelingt es Akteuren also, ihre jeweiligen Darstellungen auf plausible Weise zu vermitteln? Wie die Befunde zeigen, übertragen sich die bessere Ressourcenausstattung und der Etablierten-Status der westeuropäischen Vertreter in eine politische Dominanz gegenüber den Repräsentanten der neuen Mitgliedsländer: Die solidarische Etablierung und Durchsetzung gemeinsamer Positionen unter Bezug auf den Deutungsrahmen eines, Sozialen Europa' gelingt nur, wenn sie im Interesse der mächtigeren Akteure ist.

3) Angesichts der unterschiedlich ausgeprägten Fähigkeit zur Mobilisierung des Deutungsrahmens ,Soziales Europa' stellt sich die Frage, inwiefern solidarisches Handeln, welches auf Grundlage undemokratischer Entscheidungsprozesse zu Stande gekommen ist, eigentlich als dauerhaft stabile Grundlage kollektiven Handelns zur Verfügung steht. Abschließend wird zu argumentieren sein, dass eine deliberative Auseinandersetzung auf Augenhöhe als Grundlage eines tragfähigen europäischen Sozialmodells (d.h. ermöglicht durch organisationale Reform und Quersubventionierung der ärmeren durch die reicheren Gewerkschaften) wünschenswert, doch unrealistisch, erscheint.

Der folgende Abschnitt stellt die gewerkschaftspolitischen Herausforderungen europäischer Integration mit Blick auf das Feld der Dienstleistungsfreiheit vor dem Hintergrund der Forschungsliteratur vor. Die anschließende Darstellung der theoretischen Prämissen und des methodologischen und forschungspraktischen Designs dienen der konzeptionellen Rahmung der empirischen Befunde, welche ein abschließendes Fazit vor dem Hintergrund der Theorie interpretiert und auf weitere mögliche Forschungsfragen hin zuspitzt.

\section{Gewerkschaften und das Soziale Europa}

Im allgemeinsten Sinne lassen sich Gewerkschaften als Zusammenschlüsse zur Vertretung von Lohnabhängigeninteressen verstehen. Unter kapitalistischen Bedingungen liegt die politische Hauptaufgabe der Gewerkschaften darin, die Bedingungen zu beeinflussen, unter denen Lohnabhängige ihre Arbeitskraft verkaufen (Streeck, 2005). Traditionell fokussiert die Sozialwissenschaft in diesem Zusammenhang das institutionelle Dreieck zwischen den Interessenorganisationen von Kapital, Arbeit und dem Staat (Müller-Jentsch, 2009). Im Zuge der europäischen Integration wird diese Konstellation zunehmend in Frage gestellt. 


\subsection{Die europäische Integration als Liberalisierungsprojekt?}

Der Prozess der europäischen Einigung vollzieht sich seit den 1990er Jahren vorwiegend über die Integration nationaler Arbeits-, Finanz- und Produktmärkte in einen gemeinsamen europäischen Markt. Die liberale Grundidee, der zufolge die Erweiterung eines Marktes eine effizientere Arbeitsteilung und so effektivere Produktionsmöglichkeiten schafft, dient hierbei als normative Begründung. Gleichzeitig bedingt die Privilegierung von Maßnahmen „,negativer Integration“ (Scharpf, 1999), d.h. einer Erweiterung des Arbeitsmarktes auf Kosten nationaler Regulierungskapazitäten, das Risiko eines Abbaus arbeitsmarktpolitischer Schutzmechanismen.

Um einen hieraus resultierenden Regimewettbewerb abzuschwächen, wären im Sinne Scharpfs (ebd.) gleichzeitig Maßnahmen ,,positiver Integration“ anzustreben, welche sozialpolitische Standards im grenzüberschreitenden Maßstab gewährleisten könnten. Wie zuletzt etwa die Rechtsprechung des Europäischen Gerichtshofes (Bücker \& Warneck, 2010) oder die makroökonomische Anpassungspolitik im Zuge der Euro-Krise (Müller \& Schulten, 2013) noch einmal verdeutlicht haben, funktioniert europäische Integration bislang vorwiegend als Marktintegration. Die Europäische Union erscheint aus dieser Sicht, mit Wolfgang Streeck (2013) gesprochen, als „Liberalisierungsmaschine“.

Gleichzeitig betont Seikel (2016, S. 6) die Potenziale der europäischen Integration für eine humanzentrierte und gerechte Wirtschaftspolitik:

„Durch die Zusammenarbeit auf der europäischen Ebene könnten die Voraussetzungen für transnationale Solidarität geschaffen, der gegenseitige Unterbietungswettbewerb unterbunden, Märkte reguliert und der Vorrang des Politischen und des Demokratischen vor dem Ökonomischen wiederhergestellt werden.“

Die Auseinandersetzung um die Dienstleistungsfreiheit stellt für die Verhandlung und Etablierung einer entsprechenden institutionellen Ordnung einen zentralen Austragungsort dar.

\subsection{Die Dienstleistungsfreiheit als gewerkschaftspolitische Herausforderung}

Während der Nationalstaat traditionell die Gültigkeit von Arbeits- und Beschäftigungsstandards gewährleistet hat, wird diese Form der Ordnung im Zuge der europäischen Integration zunehmend in Frage gestellt. Die Dienstleistungsfreiheit stellt, neben freiem Waren-, Kapital- und Zahlungsverkehr sowie der Personenfreizügigkeit, in der EU ein Grundprinzip wirtschaftlicher Integration dar. Eine besondere Bedrohung des Nationalstaats als tarifpolitischer Bezugseinheit ergab sich hier in den Jahren 2004 bis 2006 im Zuge der Debatte um die Richtlinie über Dienstleistungen im Binnenmarkt (2006/123/EG) (kurz: Dienstleistungsrichtlinie). Während im Zuge der Osterweiterung, dies belegt Krings (2015, S. 91), „die Migrationsbereitschaft aus den neuen Mitgliedsstaaten tendenziell an[stieg], je höher die Lohnunterschiede zu den alten Mitgliedstaaten waren“, sah es ein Vorschlag der EUKommission vor, die Tarifstandards letzterer hierbei auf Grund des dort geforderten Herkunftslandprinzips (Art. 16; Menz, 2010, S. 977) vollkommen legal zu umgehen.

Artikel 50 des Vertrags über die Europäische Union enthält das Prinzip der NichtDiskriminierung von Dienstleistungsanbietern innerhalb der EU. Demzufolge „kann der Leistende zwecks Erbringung seiner Leistungen seine Tätigkeit vorübergehend in dem Mitgliedstaat ausüben, in dem die Leistung erbracht wird, und zwar unter den Voraussetzungen, wel- 
che dieser Mitgliedstaat für seine eigenen Angehörigen vorschreibt. “2 In diesem Sinne sollten also die Mitgliedstaaten dafür Sorge tragen, ,,dass Dienstleistungserbringer lediglich den Bestimmungen ihres Herkunftsmitgliedstaates unterfallen“" (Schilling, 2009, S. X).

Die Tatsache, dass es den europäischen Gewerkschaften durch eine groß angelegte Kampagne gelang, das Herkunftslandprinzip aus dem Gesetzestext der schließlich verabschiedeten Richtlinie zu entfernen, offenbart deren Fähigkeit zu international solidarischer Zusammenarbeit. Die Überwindung einer Konfliktlinie zwischen den Organisationen aus den alten und den neuen Mitgliedsländern im Diskussionsprozess erscheint hierbei besonders bemerkenswert: Obwohl gerade die polnischen Lohnabhängigen im Vorfeld der Kampagne zu Symbolfiguren eines mobilitätsbedingten Unterbietungswettbewerb stilisiert worden waren, fanden sich unter den 75.000 Demonstranten, die am 19. März 2005 durch Brüssel marschierten (Kowalsky, 2007, S. 147), zahlreiche Vertreter osteuropäischer Gewerkschaften. Anschließend an die Einführung in den Forschungsstand zur europäischen Gewerkschaftspolitik sowie die Vorstellung des theoretischen Rahmens behandelt diese Fragen die empirische Darstellung in Abschnitt 5.

\subsection{Gewerkschaften im Prozess der europäischen Integration}

Unter Bedingungen marktbasierter Integration der EU-Mitgliedstaaten erschwert sich auch die gewerkschaftliche Aufgabe, ,[u]nterschiedliche und teilweise konkurrierende Einzelinteressen integrativ zu organisieren“ (Dribbusch, 2014, S. 339). Die Verschiedenartigkeit der nationalen Kapitalismusmodelle haben Höpner und Schäfer (2010) auf den Begriff der ,politökonomischen Heterogenität“" gebracht, unter deren Bedingungen - so schließen die beiden - die wirtschaftliche Integration der EU zur Gefahr für ihre politische Integration wird. Die Schwierigkeiten, welche sich aus gewerkschaftlicher Sicht ergeben, wenn Organisationen aus 28 Mitgliedsländern gezwungen sind ihre politischen Ziele zu koordinieren, wurden an anderer Stelle (Seeliger, 2017) als doppeltes Heterogenitätsproblem bezeichnet: Während sich die nationalen Produktions- und Wohlfahrtssysteme der Mitgliedsländer auf einer institutionellen Ebene beispielsweise mit Blick auf die Arbeitsgesetzgebung oder den Grad sozialer Sicherung unterscheiden, finden sich zwischen den Organisationen weitere wesentliche Differenzen nicht nur mit Blick auf die politische Durchsetzungskraft, sondern auch hinsichtlich ihrer politischen Orientierung.

Gemeinsame Positionen entwickeln die europäischen Gewerkschaften innerhalb einer Abstimmungs- und Vertretungsstruktur, welche von der lokalen über die nationale bis hin zur europäischen Ebene reicht. Gegründet im Jahr 1973 von 17 Organisationen aus 15 Ländern steht der Europäische Gewerkschaftsbund (EGB) an der Spitze dieses Systems als umfassendste Vertretungsinstanz. Seine Erweiterung in den vier seitdem vergangenen Jahrzehnten auf 85 Föderationen aus 36 Ländern läuft parallel zur Zunahme der politökonomischen Heterogenität der Union.

Das politische Mandat des EGB beschreiben Reutter und Rütters (2003) als eine dreifache Aufgabenstellung: (1) Die organisationsinterne Koordination der Mitgliedsverbände (2) sowie die Interessenvertretung gegenüber EU-Institutionen und (3) europäischen Ar-

2 http://www.europarl.europa.eu/brussels/website/media/Basis/Vertraege/Pdf/Vertrag_Amsterdam_konsol.pdf (Abruf 15.12.2017). 
beitgeberverbänden. Angesichts des begrenzten Erfolges letzterer lassen sich hierbei besonders der „Institutionenbezug“ (ebd., S. 529) sowie seine ,information and coordination role" (Hyman, 2013, S. 171) hervorheben. Vor diesem Hintergrund schreibt Gajewska (2009, S. 82) dem EGB demnach eine „framing function“ zu. Indem er Agenda-Setting und öffentliche Darstellung politischer Angelegenheiten beeinflusst, wirken er und die entsprechenden Europäischen Branchenverbände maßgeblich an der Etablierung derjenigen Interpretationsrahmen mit, die kollektive Mobilisierung von europäischen Gewerkschaften ermöglichen. Als besondere Herausforderung stellt sich hierbei die Überbrückung und Mediation interner Differenzen zwischen den Mitgliedsorganisationen aus den unterschiedlichen Ländern dar. Mit Blick auf die soziale Konstruktion internationaler Solidarität fokussiert der vorliegende Beitrag das Wechselspiel zwischen der nationalen und der europäischen Ebene gewerkschaftlicher Interessenvertretung.

Im Stand der Forschung zur europäischen Gewerkschaftspolitik stellt die Diskussion um die europäische Dienstleistungsrichtlinie einen der am besten dokumentierten Fälle internationaler Zusammenarbeit dar (siehe exemplarisch Arnold, 2008; Gajewska, 2009). Die Debatte zieht ein besonderes Interesse auf sich, weil sie gemeinhin als wichtiger Präzedenzfall erfolgreicher internationaler Zusammenarbeit angesehen wird. Die Tatsache, dass die osteuropäischen Gewerkschaftsvertreter die Kampagne des EGB zur Entfernung des Herkunftslandprinzips unterstützt haben, führt Bernaciak (2011, S. 35) am Beispiel der polnischen Gewerkschaften auf drei Beweggründe zurück. Durch die Vermeidung von Unterbietungswettbewerb erhofften sie sich erstens die graduelle Angleichung ihrer nationalen Standards an die Gegebenheiten der alten Mitgliedsländer. Die Beibehaltung des Herkunftslandprinzips stellte aus ihrer Sicht zweitens eine Bedrohung für die polnischen Standards durch andere, günstigere Anbieter dar. Und drittens befürchteten sie, dass die Einführung des Prinzips die Überwachung entsprechender Standards innerhalb der EU stark verkomplizieren, wenn nicht sogar unmöglich machen würde. Diese Beweggründe, so Bernaciak (2011), motivierten die osteuropäischen Gewerkschaften schließlich zur Unterstützung der westeuropäischen Position, deren normatives Rahmenwerk traditionell das europäische Sozialmodell verkörpert.

\subsection{Das europäische Sozialmodell}

Der Bezug auf ein europäisches Sozialmodell stellt im integrationspolitischen Diskurs seit den 1970er Jahren eine bekannte Chiffre dar (Pierson \& Leibfried, 1998). Auch bekannt unter dem Begriff eines ,Sozialen Europa“ ist das Konzept „gleichermaßen analytische Kategorie, ideologisches Konstrukt und Streitgegenstand“" (Hyman, 2006, S. 121; siehe auch Delanty \& Rumford, 2005, S. 108). Die politische Essenz des Konzepts ergibt sich hierbei aus dem Vorhaben, die marktbasierte Integration durch eine sozial- und arbeitsmarktpolitische Regulation zu komplementieren. Programmatisch ist hierbei die (tendenziell sozialdemokratisch aufgeladene) Leitorientierung, ,to provide a 'human face' to the completion of the single market, and to turn the trade union movements of Europe from potential opponents into reliable allies" (Hyman, 2003, S. 27). Ein normativ und funktional begründeter gewerkschaftlicher Internationalismus liegt dem Konzept hierbei insofern zugrunde, als die politische Institutionalisierung gemeinsamer Sozialstandards nicht auf nationaler, sondern auf EU-Ebene umgesetzt werden soll. 
Mit Vaughan-Whitehead (2003, S. 4) lassen sich hierbei drei komplementäre Grundgedanken als ideelles Fundament des Konzepts identifizieren: (1) Die Wettbewerbsfähigkeit der nationalen Ökonomien ist gewährleistet und (2) benötigt hier sowohl soziale Kohäsion und Solidarität als auch (3) die demokratische Einbeziehung der europäischen Bürger: "Specific core values of this model of particular interest in the context of enlargement include democracy, individual rights, free collective bargaining, equality of opportunity for all, and social welfare and solidarity" (ebd.). Die normative Grundausrichtung ist hierbei an die Struktur der Sozialen Marktwirtschaften Westeuropas angelehnt. Hierzu gehören „ein klares Bekenntnis der Arbeiterbewegung zur parlamentarischen Demokratie, ein wohlfahrtsstaatlich garantiertes Mindesteinkommen für alle Bürger, Tarifautonomie der Gewerkschaften sowie Regierungen, die, gleich welcher politischen Zusammensetzung, sich einer aktiven Vollbeschäftigungspolitik verpflichtet fühlten“ (Streeck, 2003, S. 93).

Darüber, wie genau die hier dargestellten Zielgrößen zu verwirklichen sind, besteht in der gewerkschaftspolitischen Praxis Uneinigkeit. So bemerkt mit Kowalsky (2011, S. 94) ein Funktionär des EGB: „Das ,Soziale Europa“, die soziale Dimension Europas ist seit den Anfängen der europäischen Integration ein kontrovers diskutiertes Thema“" zwischen den Gewerkschaften. Diesen Diskussionen kommt vor dem Hintergrund der Erweiterungsrunden der Jahre 2004 und 2007 eine besondere Bedeutung zu. Denn während ein Anwachsen des Einkommens- und Wohlstandsgefälles zwischen den alten und neuen Mitgliedsländern hier einerseits den Anreiz für Lohnabhängige aus den Niedriglohnländern erhöht hat, Erwerbschancen in den Arbeitsmärkten der Hochlohnländer zu suchen, und so deren nationale Tarifsysteme unter Druck zu setzen, haben auch die Abstimmungsinitiativen im Rahmen der internationalen gewerkschaftlichen Zusammenhänge an politischer Salienz gewonnen. Der gemeinsame Bezugsrahmen eines ,Sozialen Europa' wird vor genau diesem Hintergrund und unter Beteiligung verschiedener Akteure, die jeweils unterschiedlichen Wahrnehmungen und Interessen folgen, mit substanziellen politischen Forderungen gefüllt. Der nächste Abschnitt widmet sich der Darlegung derjenigen theoretischen Prämissen, unter denen man sich im Folgenden der Frage nach einer solidarischen Zusammenarbeit europäischer Gewerkschaften im Zuge der europäischen Integration annähern kann.

\section{Theoretischer Rahmen der Untersuchung}

Ziel des vorliegenden Artikels ist es, die Frage nach einer solidarischen Kooperation europäischer Gewerkschaften im internationalen Rahmen zu beantworten. Als umfassendste Föderation kommt dem EGB hierbei der Charakter einer „Meta-Organisation“ im Sinne von Ahrne und Brunsson (2008) zu. Die interne Willensbildung sowie die Entstehung und Entwicklung vollzieht sich hier nicht zwischen Individuen oder Abteilungen, sondern wiederum zwischen eigenständigen Organisationen. Die wechselseitige Abhängigkeit, die solche Meta-Organisationen mit ihren Mitgliedseinheiten über personelle Verschränkungen, Ressourcentransfers und formelle sowie informelle Regeln verbindet, überträgt sich hier in eine dauerhafte und immer wieder aufbrechende Auseinandersetzung um Zielsetzung und Identität der Organisation. Unter eben diesen Aspekten wollen wir im Folgenden die Zusammenarbeit der Mitgliedsgewerkschaften im EGB betrachten. 
Zur Tradition der ArbeiterInnensolidarität gehört, so bemerken Bormann und Jungehülsing (2015, S. 57f.) ein „Bekenntnis zur weltweiten Inklusion“ auf Grundlage der folgenden Annahme: „Die Interessen der vom Kapital Ausgebeuteten sind weltweit prinzipiell dieselben und die Empörung über die damit verbundene Ungerechtigkeit sollte auch weltweit verbreitet sein“ (ebd., S. 58). Während die Intensität entsprechend der internationalistischen Orientierung zwischen Verbänden verschiedener (nationaler, sektoraler oder auch politischer) Provenienzen variieren mag, verpflichten sich die meisten Gewerkschaften als „wertrationale Organisationen“ (Frege \& Kelly, 2004) einer entsprechende Handlungslogik - zumindest auf rhetorischer Ebene (vgl. Höpner \& Seeliger, 2017). Unter den verschiedenen Protagonisten eines europäischen Sozialmodells im Allgemeinen, sowie den hier im für den Fall der Dienstleistungsrichtlinie untersuchten Sample vertretenen Gewerkschaften im Besonderen, lässt sich eine entsprechende Haltung bei den Vertretern aller Organisationen erkennen. Gleichzeitig jedoch „sind ihr Handlungsradius und ihre Mitgliedschaftspolitik“, wie Pernicka und Hoffmann (2015, S. 133) bemerken, ,innerhalb nationalstaatlicher Grenzen verblieben. “ Die soziale Konstruktion internationaler Solidarität vollzieht sich demnach zwangsläufig zwischen nationalen und grenzüberschreitenden Referenzrahmen.

Vor dem Hintergrund der politökomischen Heterogenität der Union ist somit davon auszugehen, dass die nationalen Mitgliedsgewerkschaften mit Blick auf die politische Zielsetzung des EGB unterschiedliche Absichten verfolgen. Ausgehend von einem Verständnis von Solidarität als einer ,soziale[n] Norm, die für eine bestimmte Gruppe gilt und deren Mitglieder dazu verpflichtet, Beiträge zu kollektiven Anstrengungen sowie zum gegenseitigen Verzicht zu leisten“ (Engler, 2015, S. 48), stellt sich also die Frage, inwiefern diese Norm bei der Formulierung und Umsetzung gemeinsamer politischer Ziele im EGB zum Tragen kommt. In diesem Zusammenhang formuliert Dribbusch (2014, S. 339) die folgende Einsicht:

„Die Internationalisierung der Wirtschaft allein bringt keinen gewerkschaftlichen Internationalismus hervor, und welche Formen internationale Solidarität annimmt, wird auch von den zugrunde liegenden Problemlagen geprägt.“

Wenn wir nun mit Gajewska (2009) davon ausgehen, dass der EGB als politischer Protagonist eines europäischen Sozialmodells eine ,framing function“ erfüllt, lässt sich die Formulierung seiner politischen Programmatik als die soziale Konstruktion eines solchen Deutungsrahmens verstehen. In Abgrenzung zu institutionalistischen Ansätzen und Theorien rationaler Wahl fokussiert eine Framing-Theorie die kognitive Komponente von Mobilisierungsprozessen. Als frames werden dabei diejenigen Deutungsrahmen verstanden, mit deren Hilfe Akteure Situationen wahrnehmen und beurteilen.

Eine politökonomische Operationalisierung eines solchen Ansatzes findet sich bei Ansell (1997, S. 359) am Beispiel der französischen Gewerkschaften, „die trotz unterschiedlicher politischer Ausrichtung gemeinsam zum Generalstreik aufriefen." Indem sich diese um das Symbol des Generalstreiks organisierten, konnten sie ihre konkurrierenden politischen Orientierungen überbrücken und eine einheitliche Position der Gewerkschaftsbewegung entwickeln. Hieraus zieht Ansell (ebd., S. 360) den Schluss, dass "organizing symbols create a shared interpretative framework that facilitates coordination, exchange, and ultimately commitment."

Um Solidarität als politische Maxime zu etablieren, so lässt sich schließen, müssten die europäischen Gewerkschaften den Frame eines europäischen Sozialmodells als gemeinsa- 
men Deutungsrahmen etablieren, der solidarisches Handeln nahe legt. $\mathrm{Zu}$ einer entsprechenden Einschätzung gelangt in seiner politischen Soziologie betrieblichen Streikhandelns auch Rick Fantasia (1988, S. 11), wenn er Solidarität als „,micro-funded process“ begreift. „Solidarity”, so Fantasia (ebd.), “is created and expressed by the process of mutual association." Die Frage nach der sozialen Konstruktion von Solidarität ist somit als handlungstheoretisches Problem zu verstehen.

Zumindest für die handlungstheoretische (d.h. interaktionistische) Perspektive ist Solidarität nicht in einem holistischen Sinne vorhanden oder abwesend. Stattdessen muss sie immer wieder in konkreten Handlungen hergestellt werden. Im Sinne von Engler (2015, S. 43) ergeben sich aus dieser Sicht „drei zentrale Dimensionen“, innerhalb derer die Entstehung und Wirksamkeit gewerkschaftspolitischer Solidarität zu untersuchen sind. Die erste dieser Dimensionen bezieht sich auf den Solidaritätshorizont, welcher die Zugehörigkeit zu der jeweiligen Solidargemeinschaft markiert. Dieser kann innerhalb verschiedener Rahmen, also beispielsweise territorial (etwa nach Nation, Region oder Kontinent), er kann aber auch funktional (d.h. nach Unternehmen oder Wirtschaftssektoren) oder kulturell festgelegt sein.

Die zweite Dimension ist die Intensität, die eine solidarische Orientierung in der politischen Praxis annimmt. Mit Blick auf die gewerkschaftliche Zusammenarbeit findet sich diese Intensität in unterschiedliche Ausprägungen. Eine erste Form bezeichnet Engler mit dem Begriff der passiven Solidarität. Diese, so Engler (ebd.) weiter, wird geübt, „wenn Schaden von Anderen abgehalten wird oder anderen Akteuren Chancen eingeräumt werden, ohne dass man diese aktiv unterstützt." Hierunter würden etwa eine klassische Solidaritätsadresse fallen, wie sie Gewerkschaften in regelmäßigen Abständen ausgeben, um ihre ideelle Unterstützung politischer Projekte zu erklären (,Der europäische Metallgewerkschaftsbund erklärt sich solidarisch mit dem Streik der Milchbauern in Nicaragua!“). Eine substanziellere Ausprägung kann sich hinsichtlich der Intensität in Form von Umverteilungssolidarität zeigen, welche im Sinne von Englers Definition zur kollektiven Anstrengung zu Gunsten der betreffenden Solidargemeinschaft führen müsste. Hiermit können etwa wirtschaftliche Einbußen oder der Verlust politischer Macht gemeint sein, welche auf Grund des solidarischen Handelns für Verantwortliche entstehen.

Eine dritte Analysedimension folgt mit Engler (ebd.) schließlich aus der Zeitdimension solidarischen Handelns. So kann sich Solidarität zum einen über einen Zeitraum hinweg sowohl in Bezug auf ihre Intensität als auch hinsichtlich ihres Horizontes entwickeln. Damit bezieht sich diese Zeitdimension auf die Dynamik, welche Engler (2015, S. 46) als „Streckbarkeit der Reziprozitätserwartungen“ beschreibt. Je nach Ausprägung der Solidarität kann der Bestand einer Solidargemeinschaft also länger oder kürzer andauern, bzw. den solidarisch Handelnden bei ihrer Antizipation des Zeitraums in der Gegenwart unterschiedliche Vorstellungen vermitteln, wie lang die Mitglieder ihrer Solidargemeinschaft Anspruch auf ihre solidarische Haltung hätten. ${ }^{3}$

3 Bemerkenswert erscheint, dass das symbolische Repertoire der Arbeiterbewegung zahlreiche quasifolkloristische Elemente aufweist, welche eine möglichst weite „Streckbarkeit der Reziprozitätserwartungen“ nahelegen. Das Proletariat, so heißt es z.B. am Ende von Marx’ und Engels’ ,Manifest der Kommunistischen Partei ‘ habe „,nichts zu verlieren als seine Ketten“, gleichzeitig aber ,eine Welt zu gewinnen“ - nur, dass das eben vermutlich eine Weile dauern würde. 
Die Frage, ob und wie die Gewerkschaften im EGB internationale Solidarität üben, entscheidet sich - so die theoretische Prämisse - abhängig davon, inwiefern die drei dargestellten Dimensionen des Solidaritätsbegriffes Eingang in den handlungsleitenden Frame eines ,Sozialen Europa' finden. Für die Aushandlung solcher Deutungsrahmen lassen sich mit della Porta (2015, S. 166) weiterhin eine Reihe (teils normativer, teils funktionaler) Prinzipien demokratischer Interessenaggregation formulieren. Erstens müssen Akteure eigene Präferenzen prinzipiell zur Disposition zu stellen bereit sein. Zweitens muss ihre Absicht gemeinwohlorientiert sein. Die Diskussion muss drittens auf Augenhöhe verlaufen, das heißt der gleiche Zugang aller Teilnehmenden zu relevanten Informationen sowie die Abwesenheit diskussionsinterner und -externer Zwänge materieller und nicht-materieller Art müssen genauso gewährleistet sein, wie eine grundsätzliche Konsensorientierung der Diskussion sowie die Einbeziehung möglichst vieler Betroffener.

\section{Methodologie und Operationalisierung}

Die empirischen Ergebnisse der hier präsentierten Studie wurden im Rahmen einer Untersuchung erhoben, welche die Positionsbildung europäischer Gewerkschaftsorganisationen im grenzüberschreitenden Maßstab zum Thema hatte. Auf Basis von insgesamt 88 Experteninterviews mit Vertreterinnen und Vertretern von Gewerkschaften aus neun europäischen Ländern sowie mit Repräsentantinnen und Repräsentanten des EGB und der europäischen Branchenverbände sowie eines viermonatigen Feldaufenthaltes am Europäischen Gewerkschaftsinstitut in Brüssel wurden diejenigen Auseinandersetzungen rekonstruiert, welche zu gemeinsamen politischen Positionen in den drei wichtigsten Feldern europäischer Tarifpolitik geführt haben (neben der Debatte um die Dienstleistungsfreiheit handelt es sich hierbei um die Initiativen grenzüberschreitender Lohnkoordinierung im Metallsektor sowie die Auseinandersetzung um einen europäischen Mindestlohn, siehe Seeliger, 2017). Die Auswahl der Interviewpartnerinnen und -partner mit Blick auf die vorliegende Darstellung erfolgt aus den jeweils wichtigsten Föderationen der drei fokussierten Länder - aus Schweden Landsorganisationen i Sverige (LO), Tjänstemännens Centralorganisation (TCO) sowie die Föderation Sveriges Akademikers Centralorganisation (SACO), aus Polen Solidarnosc, Ogólnopolskie Porozumienie Związków Zawodowych (OPZZ) und Forum sowie aus Ungarn LIGA und Magyar Szakszervezetek Országos Szövetsége (MSZOSZ). Die Interviewten kamen hierbei vor allem aus den internationalen Abteilungen und Tarifsekretariaten. Um die Auseinandersetzung im internationalen Rahmen auf angemessene Weise abzubilden, umfasst die Darstellung auch die Einschätzung von an der dargestellten Diskussion beteiligten Vertreterinnen und Vertreter anderer europäischer und nationaler Organisationen. Zu letzteren zählen zum einen die Europäische Föderation der Bau- und Holzarbeiter $(\mathrm{EFBH})$, welche aufgrund der besonderen Bedeutung von Wanderarbeit im Bausektor besonders durch die Dienstleistungsrichtlinie betroffen ist, und zum anderen der Europäische Gewerkschaftsbund als umfassendste Organisation gewerkschaftlicher Interessenvertretung auf europäischer Ebene.

Die Auswahl der drei Länder greift die weiter oben aus dem Forschungsstand hergeleitete Einsicht auf, der zufolge die europäische Integration vor allem unter Bedingungen der Oster- 
weiterung mit dem Problem der politökonomischen Heterogenität des gemeinsamen Wirtschaftsraums konfrontiert ist. Mit Blick auf die Tarifpolitik zeigt sich der starke Kontrast, den die Aufnahme der neuen Mitgliedsländer hier bedingte, auch im Vergleich mit der Süderweiterung in den 1980er Jahren. Während das Pro-Kopf-Einkommen von Griechenland und Portugal immerhin bei zwei Dritteln des damaligen EU-9-Durchschnitts lag, waren es bei den Ländern der ersten Erweiterungsrunde 2004 nur 45\% und 2007 bei Bulgarien und Rumänien dann sogar nur ein Drittel (Krings, 2015, S. 90f.). Zur Abbildung der politökonomischen Heterogenität der EU unterscheiden Rüb und Platzer (2015, S. 15): „fünf in der Arbeitsbeziehungsforschung gebräuchliche europäische Grundmodelle": Ein kooperativsozialpartnerschaftliches Modell (z.B. in Deutschland, Österreich), ein skandinavisches Modell, ein romanisch-polarisiertes Modell in Südeuropa, ein liberal-angelsächsisches Modell sowie ein transformationsgesellschaftliches Modell. Die Auswahl von Schweden, Ungarn und Polen bildet demnach nicht das gesamte Heterogenitätsspektrum ab. Die soziale Konstruktion internationaler Solidarität im Fall der Diskussion um die Dienstleistungsrichtlinie lässt sich an ihrem Beispiel dennoch untersuchen, da die Auswahl möglichst unterschiedlicher Länder darauf schließen lässt, dass hier die stärksten Interessengegensätze zu überwinden wären.

Die Diskussion um die Dienstleistungsfreiheit bildet für den Zeitraum der letzten Jahrzehnte einen der zentralen Bereiche gewerkschaftlicher Zusammenarbeit im Rahmen der EU ab. Ihre besondere politische Salienz gewann die Debatte vor dem Hintergrund der fortdauernden Vertiefung des gemeinsamen Marktes sowie - im hier untersuchten Fall der Dienstleistungsrichtlinie - angesichts der Erweiterungsrunden 2004 und 2007. Der Fokus auf die im Folgenden gemeinsam mit den institutionellen Settings ihrer Heimatländer vorzustellenden Gewerkschaften zeigt eine zentrale Konfliktlinie, welche die Diskussionen im Rahmen der genannten Politikfelder strukturiert hat. Den Verlauf dieser Debatte rekonstruiert der folgende Teil anschließend an die Darstellung der institutionellen Gegebenheiten in den untersuchten Ländern.

\section{Die soziale Konstruktion internationaler Solidarität - Empirische Befunde}

\subsection{Profile der untersuchten Länder und Organisationen}

Neben den politökonomischen Rahmenbedingungen widmet sich die folgende Darstellung der allgemeinen politischen Orientierung der jeweiligen Organisationen und illustriert weiterhin deren spezifische ,national approaches to internationalism“ (Gumbrell-McCormick \& Hyman, 2013, S. 162). Als Platzhalter des ,Nordic Model“ (Hilson, 2008) repräsentieren die schwedischen Gewerkschaften ein Spektrum der auf EU-Ebene etablierten und einflussreichen Organisationen Nordeuropas. Eine starke sozialpartnerschaftliche Orientierung stellt hierbei einen Grundbestandteil des schwedischen Systems dar. Ein anhaltend hoher Organisationsgrad der Gewerkschaften gewährleistet einen ständigen Einfluss innerhalb eines, trotz jüngerer Liberalisierungsentwicklungen (vgl. Svensson, 2013) robusten Tarifsystems, welches sich grundsätzlich durch die Nichteinmischung des Staates in die Lohnfindung auszeichnet. 
Während schwedische Gewerkschaften traditionell eine starke internationale Orientierung aufweisen, die sich etwa in einem hohen Engagement in den europäischen Föderationen abbildet, lässt sich gegenüber dem europäischen Integrationsprojekt unter schwedischen Gewerkschaften eine starke Skepsis identifizieren. Gumbrell-McCormick und Hyman (2013, S. 173) zu Folge wurden sie daher auch als "reluctant Europeans" beschrieben, deren ,belief in the superiority of the Nordic industrial relations regime" laut Götz und Haggrén (2009, S. 15) mit einer generellen "idea of the superiority of Nordic society" korrespondiere.

Demgegenüber resultiert die politische Schwäche der ungarischen und polnischen Gewerkschaftsbewegungen nicht zuletzt auch aus ihrer internen Fragmentierung. Eine starke Konzentration gewerkschaftlicher Ressourcen auf betrieblicher Ebene erschwert und verhindert hierbei in beiden Ländern die Entstehung starker nationaler Verbände, deren Fehlen sich wiederum in eine verhältnismäßig schwache Repräsentation der polnischen und ungarischen Gewerkschaften sowohl in den nationalen Arenen, als auch auf europäischer Ebene überträgt (Bernaciak, Gumbrell-McCormick \& Hyman, 2014, S. 30). Geringe Kenntnis der Brüsseler Verkehrssprachen Französisch und Englisch erschweren die Zusammenarbeit daher genauso wie die knappen finanziellen Mittel oder eine habituelle Distanz zwischen ostund westeuropäischen Vertreterinnen und Vertretern (Klemm, Kraetsch, \& Weyand, 2011).

Anders als für die schwedischen Vertreter ergeben sich für die polnischen und ungarischen Repräsentanten starke Anreize für die Orientierung an der europäischen Regulierungsebene aus der geringen Ausprägung ihrer institutionellen Macht mit dem Ziel einer graduellen Anhebung nationaler Arbeits-, Beschäftigungs- und Lohnstandards. Evidenz für eine entsprechend integrationsfreundliche Haltung findet Pleines (2008, S. 18) auf Basis einer Erhebung unter osteuropäischen Gewerkschaftern. Ca. 2/3 der Befragten geben an, dass sie die allgemeine politische Bedeutung der EU gegenüber den Nationalstaaten momentan als zu gering ansehen. Aufgrund ihrer zahlenmäßigen Unterlegenheit, einer schlechten Ressourcenausstattung und ihres Außenseiterstatus gegenüber den etablierten Westeuropäern (Elias \& Scotson, 2002) ist der Einfluss der mittel- und osteuropäischen Gewerkschaften bislang gering geblieben. Zwar sind die neu beigetretenen Organisationen formal als Vollmitglieder in die Strukturen des EGB integriert, eine „,wirkliche und umfassende Einbindung in Entscheidungsfindung und Aktivitäten bleibt jedoch eine andauernde und schwierige Aufgabe des EGB“ (Gläser, 2009, S. 231).

\subsection{Die Auseinandersetzung um die Dienstleistungsfreiheit}

Die Chronologie des Konfliktes um die Dienstleistungsrichtlinie reicht vom Ende des Jahres 2003 bis zum Ende des Jahres 2006 und fällt damit genau in den Zeitraum der Diskussion um die Osterweiterung der Jahre 2004 und 2007. Die erste Version des Vorschlages für die Richtlinie erreichte über einen persönlichen Kontakt in die Kommission einen Vertreter des schwedischen Verbandes TCO kurz vor Weihnachten. Schnell bemerkten die Schweden das Konfliktpotenzial und informierten die Repräsentanten auf der europäischen Ebene.

\footnotetext{
„And then we had a lot of work during the winter and spring. Because we saw that if some unions, Eastern unions would say that this is a very good idea, we would be very weak. It was extremely important that ETUC could say: we have all our 60 members, and even the members from Eastern Europe stand together [with us, M.S.]."
} 
Den kurzen Zeitraum bis zur offiziellen Bekanntgabe des Vorschlages nutzten die Schweden, um im EGB eine programmatische Diskussion in Gang zu bringen, in deren Rahmen sich schließlich ein internationaler Konsens über die Einhaltung nationaler Standards entwickeln konnte. Angesichts einiger ,tensions between East and West“ beschreibt ein Vertreter des schwedischen Verbandes LO die gemeinsame Position in dieser Angelegenheit: „And we managed to keep the ETUC collected around the idea that there should be no competition on wages through posted workers.“

Gleichzeitig, so erklärt ein Vertreter der von der geplanten Richtlinie besonders betroffenen Europäischen Föderation der Bau- und Holzarbeiter (EFBH), stieß man zu Beginn der Diskussion auf Widerstand der polnischen Kollegen:

„Es war damals schwierig, weil die polnischen Kollegen, ich nehme mal die, die waren am zahlreichsten und die hatten auch die größten Interessen, die polnischen Kollegen haben damals gesagt, für uns ist es wichtig, dass unsere Leute Arbeit haben. Und wenn die Arbeit finden können in Holland, Deutschland, Belgien, das finden wir gut, und selbstverständlich verdienen die nicht einen Lohn, wie die Arbeiter aus westeuropäischen Ländern.“

Innerhalb der Diskussion um das ,Soziale Europa‘ wird diese Form der Unterbietungskonkurrenz von gewerkschaftlicher Seite unter dem Begriff des ,Social Dumping ' geführt:

,[E]ven though the term regularly appears in the public discourse and in policymaking circles, it is usually used in a manner that is convenient for the individual participants, thus opening the door for misconceptions and illgrounded accusations" (Bernaciak, 2012, S. 5; siehe auch Bernaciak, 2014).

In diesem Zusammenhang berichtet ein anderer Vertreter der EFBH vom Fall eines polnischen Sekretärs im EGB, welcher zur selben Zeit offensiv für das Herkunftslandprinzip geworben habe:

„Innerhalb [des, M.S.] EGB gab es eine Schwierigkeit am Anfang, als diese Dienstleistungsrichtlinie auf dem Tisch lag, dass der damalige Sekretär. [...] Der hat in internen Besprechungen gesagt, das ist ja gar kein Problem. Europa ist freier Verkehr von Kapital und Gütern und so weiter und das ist eine weitere Stufe. Also, wo ist das Problem?“

Diese Position, führt er aus, sei jedoch von den anderen Gewerkschaftern nicht akzeptiert worden:

„Und dann haben wir ihn sehr schwer unter Druck setzen müssen, um da zu sagen, hört mal, das ist ein Problem. [...] [U]nd wir haben gezeigt, dass so eine Position, die der [...] damals hatte, dass das einfach so nicht gehen würde und dass das nicht so einfach ist. Wir haben Freizügigkeit, das gehört dazu. Also kein Problem. Farewell und jeder wird glücklich.“

Dass sich die Gewerkschaften der alten und der neuen Mitgliedsländern schließlich auf eine gemeinsame Position haben einigen könne, erklären verschiedene osteuropäische Vertreter im Einklang mit Bernaciak (2011), habe nicht zuletzt an der Entwicklung einer langfristig angelegten Sichtweise gelegen, welche sich für sie im Laufe der Diskussion ergeben habe. Auf die Frage, warum man aus dem niedrigeren Lohnniveau keinen Wettbewerbsvorteil geschlagen habe, antwortet ein Vertreter der polnischen Solidarnosc mit folgenden Worten: "But that's a very, very short perspective. It is destroying the European Social Model in the western countries. And we want that. It is not like we have some sort of social model which we export into the western countries. We have nothing." Die Bereitschaft zur Kooperation ergab sich demzufolge aus der Aussicht auf eine graduelle Angleichung der Standards im gemeinsamen Markt. 
Diese Darstellung verdeutlicht ein Wesensmerkmal des ,Sozialen Europa': Es ist - zumindest aus Sicht der osteuropäischen Gewerkschafter - ein Zukunftsversprechen. Als gemeinsames Ziel dient es der Orientierung politischen Handelns an gemeinsamen Bezugspunkten. Dies zeigt sich auch in der Darstellung eines Repräsentanten der polnischen OPZZ:

"We cannot let the EU or the businessmen or capital destroy the well-working social models in Western European countries. Or the idea of a European Social Model, as such. But we need to preserve it and make as much as we can in Poland and other European countries, to move our system close to those systems. Not to get them down to our system."

Wie die Aussagen der Interviewpartnerinnen und -partner bestätigen, erfolgt die Annäherung der Positionen im Rahmen der Verständigung über die Grundwerte des europäischen Sozialmodells, wie sie von Vaughan-Whitehead (2003) skizziert wurden. Anders als dies jedoch im Forschungsstand (vgl. Bernaciak, 2011) suggeriert wurde, erfolgt die Angleichung der Standpunkte keineswegs aus der bloßen Einsicht der Osteuropäer in den normativen Begründungszusammenhang des ,Sozialen Europa‘. Um die Angleichung der Standpunkte beider Parteien im Zeitverlauf nachzuvollziehen, müssen deren Handlungsmöglichkeiten genauer in Betracht gezogen werden. Ein Machtungleichgewicht zwischen ost- und westeuropäischen Gewerkschaften wird praktisch von allen befragten Interviewpartnerinnen und -partner, sowohl von Seiten des EGB als auch der OPZZ, bestätigt und auf die schlechte Ressourcenausstattung sowie eine mangelnde Sprachkompetenz der mittel- und osteuropäischen Verbände zurückgeführt. Die praktischen Konsequenzen dieser Asymmetrie veranschaulicht ein ehemaliger Mitarbeiter eines deutschen Verbandes mit starkem Bezug zum Politikfeld mit einem ironischen Unterton:

„Obwohl, sage ich jetzt mal, 15 der 27 Länder zu vielen Themen überhaupt nicht die Stimme erhoben haben. Aber für diese armen und eher schwachen Gewerkschaftsbewegungen erledigt man die Stimmvertretung, die Stimmabgabe gewissermaßen gleich mit. Das führt in der Summe häufig dazu, dass es so eine Gruppe von besser organisierten Ländern gibt, die eigentlich die Inhalte und die Positionen bestimmen.“

Direkte Fragen nach Machtungleichgewichten beantworteten die osteuropäischen Interviewpartnerinnen und -partner meist unter Bezug auf leicht objektivierbare Kriterien (Ressourcenausstattung, Sprachkompetenz). Gleichzeitig zeigten sich in den Gesprächsverläufen auch Anzeichen einer anders gelagerten Diskrepanz. Über die anhaltende Marginalität seiner Organisation im EGB äußert sich etwa ein ungarischer Gewerkschafter der MZSOSZ in deutscher Sprache: ,Wir sind dazugekommen. Das ist so, das ist normal. Da ist schon eine Familie und Du kommst von außen. Das ist ganz verständlich." Gleichzeitig erklärt ein Vertreter der deutschen Industriegewerkschaft Bauen-Agrar-Umwelt (IG BAU), dass viele der Kollegen aus den neuen Mitgliedsländern „Europa als solches, EU, mit all seiner Widersprüchlichkeit und Brüchigkeit eigentlich noch gar nicht richtig begriffen“ hätten.

Vor diesem Hintergrund lassen sich auch diejenigen Widersprüche und Brüche in den Darstellungen des Abstimmungsprozesses in den Schilderungen der Interviewpartnerinnen und -partner interpretieren, welche auf einen weit weniger kohärenten Verlauf dieses Prozesses hindeuten. Für die Kooperationsbereitschaft der osteuropäischen Vertreter im Fall der Dienstleistungsrichtlinie benennt etwa ein EGB-Mitglied im Gespräch zwei Motive eine materielle Komponente sowie das Interesse an einer Fortführung der Zusammenarbeit über den Fall hinaus: 
„Jetzt bei der Dienstleistungsrichtlinie haben wir denen natülich gesagt, dass es wichtig ist, dass die solidarisch sind. Aber jetzt nicht als moralischer Appell, sondern einerseits, um zu verhindern, dass wir da auseinanderdriften. Und andererseits uns nochmal mit denen zusammensetzen und die Lage nochmal zu besprechen. Und immer, wenn das sozusagen ans Eingemachte geht, um Tarifverträge, dann ist da Einigkeit. Je näher wir sozusagen an das Kerngeschäft kommen, desto eher ist es möglich, alle in einem Boot zu haben. Und je mehr wir uns davon entfernen und zu mehr ideologischen Positionen kommen, desto mehr zersplittert das und insbesondere in den Ländern, wo es dann noch ideologisch miteinander konkurrierende Gewerkschaften gibt.“

Reibung und Konflikt bei der Etablierung der Position beschreibt der Vertreter der ungarischen MSZOSZ. Ihm zu Folge wurde die Anerkennung westlicher Standards unter ungarischen Gewerkschaftern keineswegs von vornherein als Selbstverständlichkeit angesehen:

„Wir hatten auch Konflikte unter uns hier in diesem Saal, wo ich und einige angegriffen wurden von unseren Gewerkschaften, dass [...] Es ist besser, wenn man weniger als der Einheimische verdient, aber immerhin es gibt Arbeitsplatz, da kommt viel mehr als zu Hause.“

Wie sich in den Interviews mit den polnischen und ungarischen Gewerkschafterinnen und Gewerkschaftern herausstellte, fühlen sich diese in der politischen Programmatik des EGB häufig nicht repräsentiert. So berichten sowohl polnische als auch ungarische Vertreter, dass ihre Positionen in den Brüsseler Debatten nicht immer angehört würden. Eine Repräsentantin der MSZOSZ erörtert diesen Umstand wie folgt: „When I am in Brussels I mainly listen to what my colleagues from the West are saying. They have the main expertise on the topics we care about."

Angesichts der höheren Sachkenntnis der westlichen Vertreter gibt ein anderer ungarischer Vertreter an, keine Probleme mit deren Vormachtstellung zu haben: „As a person, who watched the development of the ETUC, I can accept that the old members are overrepresented in the organization. Because the European countries are somehow close into the system, which we get."

Dementsprechend zieht auch ein polnischer Vertreter des EGB den kompakt formulierten Schluss, das ,Soziale Europa“ sei "pretty much a western idea“, entwickelt von westeuropäischen Vertretern mit westeuropäischen Maßstäben. Eine ähnliche Einschätzung äußert ein ehemaliger Mitarbeiter der EFBH:

„Ja, ich glaube schon, dass das sogenannte ,Soziale Europa‘, das es ja eigentlich nicht gibt, dass das natürlich ein Modell war. Das stimmt schon. Das ist ein Modell der westeuropäischen Länder und der westeuropäischen Politik. Da waren ja die Osteuropäer gar nicht dran beteiligt."

Dieser ungleichen Beteiligung schreibt der polnische EGB-Vertreter eine grundlegende Bedeutung für die Entwicklung der EU zu: "In fact, the whole integration process was like that. You have never had this kind of negotiation on equal footing. It was the matter of joining the block with some rules." Etabliertenvorrechte und Ressourcendiskrepanz bedingten auch aus Sicht eines Vertreters der ungarischen MSZOSZ eine anhaltende Konstellation, innerhalb derer die Osteuropäer systematisch unterlegen seien:

„Das ist überhaupt nicht balanciert, wir [die Osteuropäer, M.S.] sind Neulinge. Obwohl, wenn man 20 Jahre dabei ist, ist man schon nicht mehr Neuling. Aber auf unserer Seite sehen wir, dass wir schwach sind. Wir sind schwach allgemein und haben auch kaum echte Ressourcen, alle Politikbereiche wirklich zu bearbeiten und offensiv in die Diskussionen reinzugehen."

Die politische Tragweite dieser fallspezifischen Auseinandersetzung ergibt sich schließlich aus der Erklärung eines Vertreters der schwedischen LO, demzufolge unter europäischen 
Gewerkschaftern wenig abstrakte Diskussionen geführt würden: „It is quite seldom that we debate on principles. Debates are always on issues that happen“. Europäische Gewerkschaftspolitik gewinnt ihre Programmatik daher aus der Praxis. Ihren grundsätzlichen Charakter nimmt die Auseinandersetzung um die Dienstleistungsrichtlinie daher durch ihre Einbindung in die konzeptionelle Diskussion um ein Soziales Europa.

\section{Zusammenfassung}

Die Rekonstruktion der gewerkschaftlichen Positionsbildung zur Dienstleistungsrichtlinie zeigt, wie es den westeuropäischen Vertreterinnen und Vertretern gelungen ist, ihre osteuropäischen Kolleginnen und Kollegen von einer offensiven Unterstützung internationaler Unterbietungskonkurrenz abzuhalten. Nach einigen Diskussionen konnten die beteiligten Personen Einvernehmen über die Zustimmung zum Konzept eines europäischen Sozialmodells auf Basis allgemein akzeptierter Mindeststandards generieren. Am Ende der Diskussion stand also ein gemeinsamer Bezugsrahmen politischer Mobilisierung, welche dann auch tatsächlich in die Forderung nach einheitlichen Beschäftigungsbedingungen überführt werden konnte. Mit der Einwilligung in die Notwendigkeit nord- und westeuropäischer Sozialstandards verbinden die osteuropäischen Vertreterinnen und Vertreter die Hoffnung, so in ihren Ländern mittelfristig dieselben Standards entwickeln zu können. Die Zustimmung der osteuropäischen Repräsentantinnen und Repräsentanten erfolgte hierbei gleichzeitig solidarisch und eigeninteressiert. Dass sie die Position jedoch überhaupt angenommen hatten, setzte die machtvolle Einflussnahme durch die westeuropäischen Funktionärinnen und Funktionäre voraus. Die soziale Konstruktion von Solidarität erfolgt hier also nicht vollkommen freiwillig, sondern unterliegt einem machtgebundenen Aushandlungscharakter.

\section{Fazit}

Vor dem Hintergrund der Vertiefung und Erweiterung der EU-Integration, so der Ausgangspunkt des vorliegenden Artikels, sind die Gewerkschaften Europas ,under considerable pressure" (Bernaciak et al., 2014, S. 5) geraten. Mit Blick auf die Umgangsform, die die Gewerkschaften als potenzielle Repräsentantinnen eines gemeinsamen Klasseninteresses der europäischen Lohnabhängigen vertreten, waren die hier angestellten Überlegungen geleitet von der „Vermutung, dass in Europa zunehmend häufiger soziale Konflikte eines neuen, transnationalen Typs auftreten, bei denen es um das Aushandeln einer europäischen Solidaritätsnorm geht" (Engler, 2014, S. 15).

Diese Frage haben wir unter Bezug auf die Mobilisierung des Deutungsrahmens eines ,Sozialen Europa' behandelt. Dieser Rahmen, so zeigte die Analyse der gewerkschaftspolitischen Debatte um die Dienstleistungsrichtlinie unter besonderer Berücksichtigung von Organisationen aus Schweden, Polen und Ungarn, lässt sich in unterschiedlicher Weise zur Koordinierung politischen Handelns mobilisieren. Die in diesem Zusammenhang gewonnenen Erkenntnisse lassen sich abschließend zu drei Befunden zuspitzen.

Auf der allgemeinsten Ebene zeigt die handlungs- und framing-theoretische Darstellung der Geschehnisse in den Feldern, wie (un)solidarische Haltungen politischer Akteure als Resultat (mitunter konfliktiver) Aushandlung über Möglichkeit und Notwendigkeit eben 
dieser Haltungen entstehen. Im Einklang mit bereits vorliegenden Befunden (z.B. Fantasia, 1988) bestätigt sich daher der grundsätzlich plastische und prozedurale Charakter von Solidarität als sozialem Konstrukt. Die soziale Konstruktion internationaler Solidarität erfolgt hierbei in Form der Etablierung eines Deutungsrahmens, welcher sich in diesem Fall aus der konkreten Ausgestaltung der politischen Implikationen eines europäischen Sozialmodells ergibt. Im Einklang mit Bernaciak (2011), können wir auf Grundlage der Befunde feststellen, dass international solidarische und national eigeninteressierte Orientierungen bei den polnischen und ungarischen Vertretungen in der politischen Praxis eine Synthese eingehen. Diese erfolgt jedoch - anders als Bernaciak dies darstellt - nicht auf Grund rein rationaler Überlegungen, sondern als Resultat eines machtgebundenen Aushandlungsprozesses.

Der zweite Befund bezieht sich hierbei auf die sozialen Dynamiken, welche den gemeinsamen Deutungsrahmen hervorbringen: Die bessere Ressourcenausstattung und der Etablierten-Status der westeuropäischen Vertreterinnen und Vertreter übertragen sich in eine politische Dominanz entlang der Differenz zwischen Etablierten und Außenseitern: Die solidarische Etablierung und Durchsetzung gemeinsamer Positionen unter Bezug auf den Deutungsrahmen eines ,Sozialen Europa' gelingt also, wenn sie im Interesse der mächtigeren Akteure ist. Die schwedische Position lässt sich im Falle der Dienstleistungsrichtlinie also anschließend an Streeck (1995, S. 120) als ,institutioneller Nationalismus“ beschreiben, welcher

„soweit wie möglich an den bestehenden nationalen Organisationsformen festhalten, bzw. Internationalisierung nur in dem Maße akzeptieren will, wie sie die Stabilität und Autonomie nationaler Institutionen nicht beeinträchtigt und national definierte Interessen nicht benachteiligt.“

Eine verschieden stark ausgeprägte Fähigkeit zur Frame-Mobilisierung im Rahmen des EGB ergibt sich für die beiden Parteien aus drei Ursachen: Zum einen bedingen geringere Mitgliedschaftszahlen und niedrigere Löhne in den Beitrittsländern ein Gefälle im Hinblick auf Abstimmungsmacht und monetäre Möglichkeiten (z. B. zur Akquise externen Expertenwissens oder der Deckung von Reisekosten). Eine zweite Ursache liegt in den etablierten Verhandlungslogiken der Brüsseler Gewerkschaftspolitik, welche überwiegend dem Muster der koordinierten Marktwirtschaften Westeuropas entsprechen. In der Wahrnehmung der Osteuropäerinnen und -europäer sind diese im Wesentlichen vor die Wahl gestellt, mit den Auffassungen der westeuropäischen Vertreterinnen und Vertreter übereinzustimmen oder sich zurückzuhalten. Eine dritte Ursache folgt schließlich aus einer Etablierten-Außenseiter-Dynamik zwischen den Vertreterinnen und Vertretern der alten und der neuen Länder. Neben etablierten Netzwerkbeziehungen und technisch-administrativem Know-how ergibt sich die Diskrepanz auch aus den Sprachproblemen der osteuropäischen Gewerkschafter. Die hier genannten Machtasymmetrien nutzen die etablierten Vertreterinnen und Vertreter der alten Mitgliedsländer nun, um die Parameter der Diskussion so zu definieren, dass der Schutz ihrer nationalen Beschäftigungsstandards als Substanz des europäischen Sozialmodells plausibilisiert und gewährleistet ist.

Drittens stellt die Abstimmung zwischen den Gewerkschaftsvertreterinnen und vertretern über die Zusammenarbeit - unter Bezug auf della Portas (2015, S. 165) Modell deliberativer Abstimmung - ein normatives Problem dar. Zwar verlief die Diskussion insgesamt transparent. Die Rahmenbedingungen politökonomischer Heterogenität bedingten jedoch eine Konstellation, in der der Maßstab des politischen Handelns sich nicht aus den 
Erfahrungen der Mittel- und Ost-, sondern denen der Nord- und Westeuropäerinnen und europäer ergibt. Die von Vaughan-Whitehead (2003, S. 8) angeführten Grundprinzipien des 'Sozialen Europa' einer "participation extended to all citizens" sowie einer "inclusive society" erscheinen vor diesem Hintergrund jedenfalls nicht gewahrt. Insgesamt ist damit Gläsers (2009, S. 231) Einschätzung zuzustimmen, der die Statusasymmetrien innerhalb des EGB zu Lasten der osteuropäischen Vertreter beklagt.

Es stellt sich hieran anschließend die Frage, ob dieser Asymmetrie mittel- und langfristig durch Maßnahmen finanzieller Quersubventionierung oder transnationaler Capacity BuildingInitiativen begegnet werden könnte (vgl. Bernaciak et al., 2014, S. 82). Inwiefern entsprechende Strategien Grundlage der Fortentwicklung eines europäischen Sozialmodells sein könnten stellt einen möglichen Gegenstand zukünftiger Untersuchungen zum Thema dar.

Mit Blick auf die Entwicklungsperspektiven eines solchen ,Sozialen Europa ‘ beklagen Eigmüller und Mau (2010, S. 12) die Tatsache, dass sowohl in der politischen, als auch in der fachlichen Diskussion ,zumeist mit Projektionen eines zukünftigen Europas argumentiert wird." Diesem Umstand habe ich in der vorliegenden Untersuchung zu begegnen versucht, indem ich die solidarische Programmatik des europäischen Sozialmodells mit dessen realpolitischen Praktiken kontrastiert habe. Eine theoretisch-konzeptionelle Schwachstelle, dies zeigt sich in Auseinandersetzung mit dem Modell Englers (2014, S. 47), ergibt sich hierbei aus der grundlegenden Zukunftsorientierung politischen Handelns. Denn wenn die EU, wie Engler dies selbst konstatiert, ein Beispiel für „,den längerfristigen (unintendierten) Aufbau von Solidarität“ ist, so können wir die Intensität dieser Solidarität als solche nur rückwirkend untersuchen.

In Bezug auf zukünftige Solidaritätserfordernisse lässt sich eine entsprechende Orientierung unter den politischen Akteuren jedoch lediglich als eine Antizipation derselben erkennen - welchen Wettbewerbsvorteil ein ,race to the bottom' den ungarischen oder polnischen Gewerkschafterinnen und Gewerkschaftern tatsächlich erschlossen hätte, können wir nicht wissen. Es zeigt sich hier also abschließend erneut die Zeit- und Prozessdimension von Solidarität, deren Wirksamkeit in der Gegenwart mittelfristig auf einem Zukunftsversprechen beruhen muss. Solche Mobilisierungsnarrative ließen sich aus Sicht einer politischen Soziologie - etwa im Sinne Beckerts (2016) als fiktionale Erwartungen untersuchen. Auf Grundlage der Befunde dieser Studie wäre damit zu erwarten, dass sich verhältnismäBig schwache Akteure eher überzeugen ließen, andere Interessen zu übernehmen als verhältnismäßig starke. Nicht nur im Bereich der internationalen Gewerkschaftspolitik ließen sich Fälle finden, anhand derer diese Frage weiter untersucht werden könnte.

\section{Literatur}

Ahrne, G. \& Brunsson, N. (2008). Meta-organizations. Cheltenham: Edward Elgar. doi: $10.4337 / 9781848442658$

Ansell, C. (1997). Symbolic Networks: The Realignment of the French Working Class, 1887-1894. American Journal of Sociology, 103 (2), 359-390. doi: 10.1086/231211

Arnold, L. M. (2008). Die Entstehung der europäischen Dienstleistungsrichtlinie im Spannungsfeld organisierter Interessen: Eine Fallstudie zum Einfluss von Gewerkschaften und Unternehmerverbänden im Europäischen Parlament. Hertie School of Governance - working papers, No. 36.

Beckert, J. (2016). Imagined Futures. Cambridge: Harvard. doi: 10.4159/9780674545878 
Bernaciak, M. (2011). East-West European Labour Transnationalism(s). In A. Bieler \& I. Lindberg (Hrsg.), Global Restructuring, Labour and the Challenges for Transnational Solidarity (S. 3347). London: Routledge.

Bernaciak, M. (2012). Social dumping: political catchphrase or threat to labour standards? Brussels: ETUI Working Paper 2012/06.

Bernaciak, M. (2014). Social dumping and the EU integration process. Brussels: ETUI Working Paper 2014/06.

Bernaciak, M., Gumbrell-McCormick, R. \& Hyman, R. (2014). European trade unionism: from crisis to renewal? Brussels: ETUI Report 133.

Bormann, S. \& Jungehülsing, J. (2015). Einleitung: Transnationale Solidarität als Prozess denken. In S. Bormann, J. Jungehülsing, S. Bian, M. Hartung \& F. Schubert (Hrsg.), Last Call for Solidarity. Perspektiven grenzüberschreitenden Handelns von Gewerkschaften (S. 15-39). Hamburg: VSA.

Bücker, A. \& Warneck, W. (Hrsg.). (2010). Viking - Laval - Rüffert: Consequences and policy perspectives. Brussels: ETUI.

Delanty, G. \& Rumford, C. (2005). Rethinking Europe: Social Theory and the Implications of Europeanization. London: Routledge. doi: 10.4324/9780203640050

della Porta, D. (2015). Spreading Protest: Social Movements in Times of Crisis. Colchester: ECPR Press.

Dribbusch, Heiner (2014): Voraussetzungen internationaler Solidarität: zur Diskussion um einen europäischen Generalstreik. WSI Mitteilungen, 67 (5), 337-344.

Eigmüller, M. \& Mau, S. (2010). Gesellschaftstheorie und Europapolitik. Eine Einleitung. In: M. Eigmüller S. \& Mau (Hrsg.), Gesellschaftstheorie und Europapolitik (S. 9-32). Wiesbaden: Springer: VS. doi: 10.1007/978-3-531-92008-5

Elias, N. \& Scotson, J. L. (2002). Etablierte und Außenseiter. Frankfurt a.M.: Suhrkamp.

Engler, M. (2015). Zur Entstehung europäischer Solidarität. Wiesbaden: Springer.

Fantasia, R. (1988). Cultures of Solidarity. Berkeley: University of California Press.

Frege, C. \& Kelly, J. (Hrsg.) (2004). Varieties of Unionism: Strategies for Union Revitalization in a Globalizing Economy. Oxford: Oxford University Press. doi: 10.1093/acprof:oso/9780199270149.001.0001

Gajewska, K. (2009). Transnational Labour Solidarity: Mechanisms of Commitment to Cooperation Within the European Trade Union Movement. London: Routledge. doi: 10.4324/9780203878354

Gläser, C. (2009). Europäische Einheitsgewerkschaft zwischen lähmender Überdehnung und umfassender Repräsentativität: EGB-Strukturen und die Herausforderung der Erweiterung. Mitteilungsblatt des Instituts für soziale Bewegungen, 42, 215-234.

Götz, N. \& Haggren, H. (2009). Introduction. transnational Nordic alignment in stormy waters. In N. Götz H. \& Haggren (Hrsg.), Regional Cooperation and International Organizations (S. 1-23). London: Routledge.

Gumbrell-McCormick, R. \& Hyman, R. (2013). Trade Unions in Western Europe. Hard Times, Hard Choices. Oxford: Oxford University Press. doi: 10.1093/acprof:oso/9780199644414.001.0001

Hilson, M. (2008). The Nordic Model: Scandinavia since 1945. London: Reaktion books.

Höpner, M. \& Schäfer, A. (2010). Grenzen der Integration - wie die Intensivierung der Wirtschaftsintegration zur Gefahr für die politische Integration wird. Integration, 33 (1), 3-20.

Höpner, M. \& Seeliger, M. (2017). Transnationale Lohnkoordinierung zur Stabilisierung des Euro? Gab es nicht, gibt es nicht, wird es nicht geben. Köln: Discussion Papers. Reihe des Max-PlanckInstituts für Gesellschaftsforschung.

Hyman, R. (2003). 'Trade Unions and the Ambiguities of Social Europe', Paper presented at the IIRA Conference, Berlin, 7-12 September. 
Hyman, R. (2006). Strukturierung des transnationalen Raumes. Kann Europa dem multinationalen Kapital die Stirn bieten? In U. Brinkmann, K. Krenn S. \& Schief (Hrsg.), Endspiel des kooperativen Kapitalismus? Institutioneller Wandel unter den Bedingungen des marktzentrierten Paradigmas (S. 121-168). Wiesbaden: Springer VS.

Hyman, R. (2013). European Trade Unions and the Long March through the Institutions. From Integration to Contention? In: P. Fairbrother (Hrsg.), Transnational Trade Union. Building Union Power (S. 161-179). London: Routledge.

Klemm, M. Kraetsch, C. \& Weyand, J. (2011). „Das Umfeld ist bei ihnen völlig anders“. Kulturelle Grundlagen der europäischen betrieblichen Mitbestimmung. Berlin: Sigma. doi: $10.5771 / 9783845269580$

Kowalsky, W. (2007). Outcome of the battle on the Service Directive. Transfer, 13 (1), 147-149. doi: $10.1177 / 102425890701300112$

Kowalsky, W. (2011). Gewerkschaften für einen Kurswechsel in Europa. Gewerkschaften und Europa vor der Trennung. In W. Kowalsky \& P. Scherrer (Hrsg.), Gewerkschaften für einen europäischen Kurswechsel (S. 12-130). Münster: Westfälisches Dampfboot.

Krings, T. (2015). Bewegungsfreiheit in einem Ungleichheitsraum: Gewerkschaften und transnationale Arbeitsmobilität in der erweiterten Europäischen Union. In S. Pernicka (Hrsg.), Horizontale Europäisierung im Feld der Arbeitsbeziehungen (S. 87-110). Wiesbaden: Springer VS.

Menz, G. (2010). Are you being served? Europeanizing and re-regulating the single market in services. Journal of European Public Policy, 17 (7), 971-987. doi: 10.1080/13501763.2010.499226

Müller-Jentsch, W. (2009): Arbeit und Bürgerstatus. Studien zur sozialen und industriellen Demokratie. Wiesbaden: Springer VS.

Müller, T. \& Schulten, T. (2013). Ein neuer europäischer Interventionismus? Die Auswirkungen des neuen Systems der europäischen Economic Governance auf Löhne und Tarifpolitik. Wirtschaft und Gesellschaft, 39 (3), 291-320.

Pernicka, S. \& Hofmann, J. (2015). Ein soziales Europa? Möglichkeiten und Grenzen transnationaler Solidarität von Gewerkschaften. Zeitschrift für Politik. Sonderband Solidarität. 133-162.

Pierson, P. \& Leibfried S. (1998). Mehrebenen-Politik und die Entwicklung des ,Sozialen Europa'. In P. Pierson \& S. Leibfried (Hrsg.), Standort Europa. Sozialpolitik zwischen Nationalstaat und europäischer Integration (S. 11-57). Frankfurt a.M.: Suhrkamp.

Pleines, H. (2008). Already Arrived in Brussels? Interest Representation of Trade Unions from the New EU Member States at the EU Level. Arbeitspapiere und Materialien No. 91. Bremen: Forschungsstelle Osteuropa Bremen.

Reutter, W. \& Rütters, P. (2003). Internationale und europäische Gewerkschaftsorganisationen: Geschichte, Struktur und Einfluss. In W. Schröder \& B. Wessels (Hrsg.), Die Gewerkschaften in Politik und Gesellschaft der Bundesrepublik Deutschland: Ein Handbuch (S. 512-542). Wiesbaden: Westdeutscher Verlag. doi: 10.1007/978-3-322-80389-4_21

Rüb, S. \& Platzer, H.-W. (2015). Europäisierung der Arbeitsbeziehungen im Dienstleistungssektor. Empirische Befunde, Probleme und Perspektiven eines heterogenen Feldes. Berlin: Sigma. doi: $10.5771 / 9783845268255$

Scharpf, Fritz W. (1999). Governing in Europe: Effective and Democratic? Oxford: Oxford University Press.

Schilling, P. (2009). Neuland EU-Dienstleistungsrichtlinie. Eine strategie- und lösungsorientierte Übersicht für Unternehmen, Behörden und deren IT-Berater. Berlin: Beuth Verlag.

Seeliger, M. (2017). Die soziale Konstruktion organisierter Interessen. Gewerkschaftliche Positionsbildung auf europäischer Ebene. Frankfurt a.M./New York: Campus.

Seikel, D. (2016). Ein soziales und demokratisches Europa? Hindernisse und Handlungsperspektiven. WSI-Mitteilungen, 69 (1), 5-13. 
Streeck, W. (1995). Politikverflechtung und Entscheidungslücke. In K. Bentele, B. Reissert \& R. Schettkat (Hrsg.), Die Reformfähigkeit von Industriegesellschaften. Fritz W. Scharpf - Festschrift zu seinem 60. Geburtstag (S. 101-130). Frankfurt a.M./New York: Campus.

Streeck, W. (2003). Gewerkschaften in Westeuropa. In W. Schroeder \& B. Wessels (Hrsg.), Die Gewerkschaften in Politik und Gesellschaft der Bundesrepublik Deutschland (S. 86-100). Wiesbaden: Westdeutscher Verlag. doi: 10.1007/978-3-322-80389-4_4

Streeck, W. (2005). The Sociology of Labor Markets and Trade Unions. In N. J. Smelser \& R. Swedberg (Hrsg.), The Handbook of Economic Sociology (S. 254-283). Princeton: Princeton University Press.

Streeck, W. (2013). Gekaufte Zeit. Die vertagte Krise des demokratischen Kapitalismus. Berlin: Suhrkamp.

Svensson, T. (2013). Sweden. In C. Frege \& P. Kelly (Hrsg.), Comparative Employment Relations in the Global Economy (S. 227-244). London: Routledge.

Vaughan-Whitehead, D. (2003). EU Enlargement versus Social Europe? The uncertain Future of the European Social Model. Cheltenham: Edward Elgar. 


\section{Klaus Kock, Edelgard Kutzner}

\section{Arbeit als kollegiales Handeln - Praktiken von Solidarität und Konkurrenz am Arbeitsplatz**}

\section{Zusammenfassung}

Im vorliegenden Beitrag wird gezeigt, dass sich parallel und im Widerspruch zu konkurrenzbedingten Fragmentierungen im kooperativen Arbeitsprozess immer auch Gelegenheiten für wechselseitige zwischenmenschliche Bindungen und Verpflichtungen ergeben. Es werden Praktiken analysiert, mit denen Arbeitende ihre sozialen Beziehungen interaktiv in reziproken Austauschprozessen gestalten und zu Kollegialität im Sinne eines Füreinander-Einstehens formen. Diese Prozesse sind voraussetzungsvoll und werden ständig von gegenläufigen Prozessen der Ausgrenzung und gegenseitigen Instrumentalisierung durchkreuzt. Kollegialität beruht auf der Einsicht, dass die Realisierung eigener Ziele von der Realisierung der Ziele anderer abhängig ist, dass die eigene Handlungsfähigkeit in der Zusammenarbeit und im reziproken Austausch mit anderen erweitert werden kann. Kollegialität als eine Form von Solidarität entsteht in unmittelbaren Arbeitszusammenhängen weder aus einer gegebenen Gemeinschaft noch aus funktionalen Erfordernissen des Arbeitsprozesses. Kollegialität wird vielmehr in Interaktionen generiert und - wenn sie Bestand haben soll - immer wieder gegen Konkurrenz und konkurrentes Handeln durchgesetzt. Durch solidarisches Handeln gewinnen die Arbeitenden an Handlungsfähigkeit, deren Erweiterung auf überbetriebliche gesellschaftliche Verhältnisse weiterer Reflexion und kollektiver Aktion bedarf.

Schlagwörter: Kollegialität, Solidarität, Kooperation, Reziprozität

* Dr. Klaus Kock, TU Dortmund, Sozialforschungsstelle, Evinger Platz 17, 44339 Dortmund.

Email: kock@sfs-dortmund.de

Dr. Edelgard Kutzner, TU Dortmund, Sozialforschungsstelle, Evinger Platz 17, 44339 Dortmund.

Email: kutzner@sfs-dortmund.de

** Artikel eingegangen: 30.4.2018, revidierte Fassung akzeptiert nach doppelt-blindem Begutachtungsverfahren: 22.8.2018 


\title{
Work as Collegial Action - Practices of Solidarity and Competition in the Workplace
}

\begin{abstract}
In this article, it is shown that in parallel as well as in contradiction to competitive fragmentation in the cooperative work process there are always opportunities for mutual interpersonal ties and obligations. Practices are analyzed in which workers organize their social relations interactively in reciprocal exchange processes and collegiality, which in turn helps create a sense of a mutual support and willingness to "stand up for each other". However, these processes are full of prerequisites that are constantly thwarted by opposing processes of exclusion and mutual instrumentalization. Collegiality is based on the insight that the realization of one's own goals depends on the realization of the goals of others, that one's own ability to act can be extended in cooperation and in reciprocal exchange with others. Collegiality as a form of solidarity in direct work contexts arises neither from a given community nor from the functional requirements of the working process. Rather, collegiality is generated in interactions and - if it is to last - needs to be enforced against competition and competitive action. Through solidaristic action workers gain the ability to act. Its expansion, though, to an inter-company level will require further reflection and collective action.
\end{abstract}

Keywords: Collegiality, solidarity, cooperation, reciprocity (JEL: J5)

\section{Einleitung}

„Schluss mit Homeoffice: IBM holt die Mitarbeiter zurück ins Büro“, meldete die FAZ im Mai 2017. Dem Bericht zufolge hatte das Unternehmen die Beschäftigten vor die Wahl gestellt, in Zukunft entweder im IBM-Büro zu arbeiten oder das Unternehmen zu verlassen. Die Marketing-Chefin sei der Ansicht, „dass Leute schlagkräftiger, kreativer und wirkungsvoller sind, wenn sie Schulter an Schulter arbeiten. Und mehr Spaß mache es hoffentlich auch“ (Petersdorff, 2017). Die Firma folgt hier offenbar einem Trend zurück zu Formen der Arbeitsorganisation, in denen Präsenz und Zusammenarbeit am Arbeitsplatz als Ressourcen genutzt werden sollen.

Dieser Trend steht im Zusammenhang einer zeithistorischen Entwicklung im betrieblichen Umgang mit menschlicher Arbeitskraft, die in der Arbeits- und Industriesoziologie als „Subjektivierung von Arbeit“ bezeichnet wird. Während Subjektivität menschlicher Arbeit in tayloristischen Organisationsformen als Störfaktor galt, wird sie nunmehr als Potenzial betrachtet, das zu erschließen sei. „Gefragt ist das elastische Potenzial des sich selbst steuernden autonomen Subjekts, welches in der Lage ist, intentional zu handeln, den eigenen Arbeitsprozess selbst zu strukturieren, kreativ auszugestalten, innovativ zu sein und Verantwortung zu übernehmen“ (Lohr, 2017, S. 281-282).

Diesem Leitgedanken folgend wird auch die Gestaltung der Zusammenarbeit den Arbeitenden zunehmend selbst überlassen. Die Kontrollbestrebungen des Managements erfahren einen Formwandel. Im tayloristischen Konzept soll die Verknüpfung einzelner Teilarbeiten durch formale Regelungen und Vorschriften hergestellt werden, wenn sie nicht schon als technisch vorgegeben gilt. In neuen Organisationsformen von Arbeit wird den Beschäftigten mehr Verantwortung für ihre Zusammenarbeit überlassen, zugleich aber ver- 
sucht, den Kontext festzulegen, die Arbeitsprozesse informationstechnisch zu durchdringen und mittels Kennziffern auf das gewünschte Ziel zu lenken (Bolte \& Porschen, 2006, S. 6567).

Kooperation im umfassenden Sinne von gemeinsamer Aufgabenbewältigung, Planung, Koordination und Abstimmung der Einzelaufgaben wird zur Arbeitsaufgabe für die Beschäftigten (Bolte, Neumer \& Porschen, 2008, S. 23-24). Entsprechend den Anforderungen der konkreten Situation soll das Zusammenwirken im Arbeitsprozess von ihnen selbst organisiert werden. In der Arbeitssoziologie ist dafür der Begriff des ,erfahrungsgeleitetsubjektivierenden kooperativen Arbeitshandelns“ geprägt worden: „Die Kooperation ergibt sich aus den konkreten Erfahrungen in der alltäglichen Arbeit. Hierbei spielen subjektive Faktoren wie komplexe sinnliche Wahrnehmungen, gemeinsame Erfahrungen und Erlebnisse sowie persönliche Beziehungen eine wichtige Rolle“ (ebd., S. 52).

Mit den auf diese Weise erweiterten Handlungsspielräumen in zugleich strikter reglementierten Rahmenbedingungen ergeben sich neue Anforderungen an die Arbeitenden, einerseits betriebswirtschaftlich verantwortlich zu handeln und andererseits ihre sozialen Beziehungen untereinander zu gestalten. Die Kooperation erfordert jetzt, wechselseitig zu berücksichtigen, wie die anderen handeln, welche Erwartungen bestehen, welche persönlichen Eigenheiten bei der Arbeit zum Tragen kommen. Empathie, Verantwortung und Vertrauen werden zu notwendigen Bestandteilen selbsttätiger Kooperation (ebd., S. 127-128). Empirische Studien weisen nach, dass hiermit auch neue, anders gelagerte Probleme entstehen. Die Verschränkung von zwischenmenschlichen Beziehungen mit betriebswirtschaftlicher Kalkulation im Rahmen enger Zielvorgaben kann zu Konflikten führen, die nicht mehr mit den Vorgesetzten, sondern unter den Beschäftigten selbst ausgetragen werden und bis ins Persönliche reichen (Böhle \& Bolte, 2002; Krömmelbein, 2004; Bolte et al., 2008).

Im Unterschied und ergänzend hierzu soll im Folgenden gezeigt werden, dass der kooperative Arbeitsprozess trotz fremdbestimmter funktionaler Notwendigkeiten immer auch Möglichkeiten zur selbstbestimmten Entwicklung von Kollegialität beinhaltet, die hier als eine Form der Solidarität betrachtet wird. „To work is to work with another and for another. Both aspects bring us into the semantic domain of solidarity" (Smith, 2014, S. 168).

Kollegialität im Sinne eines Füreinander-Einstehens basiert auf der Zusammenarbeit am Arbeitsplatz. Im praktischen Vollzug gemeinsamer Aufgabenbewältigung entwickeln die Arbeitenden soziale Bindungen, gegenseitige Verpflichtungen und Vertrauen. Zugleich und im Widerspruch zu individuellen Nutzenerwägungen, wie sie im Lohnarbeitsverhältnis strukturell angelegt sind, folgt Kollegialität Motiven zwischenmenschlicher Bindung und Unterstützung. Wie im Folgenden gezeigt werden soll, ist die Entwicklung von Kollegialität ein durchaus voraussetzungsvoller Prozess, der nicht spontan aus dem Zusammenwirken erwächst, sondern bewusst gestaltet wird und immer wieder gegen vermeintliche Sachzwänge, zuwiderlaufende Regelungen und Anweisungen durch Vorgesetzte, aber auch gegen Tendenzen zu konkurrentem Handeln unter den Beschäftigten selbst durchgesetzt werden muss. Wenn dies gelingt, gewinnen die Arbeitenden an Handlungsfähigkeit nicht nur zur Aufgabenbewältigung, sondern auch darüber hinaus zur Durchsetzung gemeinsamer Interessen.

Im Folgenden wird zunächst das theoretische „Handwerkszeug“ vorgestellt. Kollegialität wird begrifflich gefasst als eine Form der Solidarität, die aus der Praxis reziproker Aus- 
tauschprozesse im Betrieb resultiert. Es folgt die empirische Analyse einiger Praktiken zur Erzeugung von Kollegialität. Im Schlussabschnitt werden Überlegungen angestellt über strukturelle Voraussetzungen der Entwicklung von Kollegialität sowie über Möglichkeiten ihrer Ausweitung auf arbeitsplatz- und betriebsübergreifende Solidarisierungsprozesse zur Entwicklung kollektiver Handlungsfähigkeit.

\section{Kollegialität als Form der Solidarität - theoretischer Hintergrund}

In diesem Abschnitt werden die theoretischen Begriffe erläutert, mit denen in Abschnitt 3 empirische Untersuchungsergebnisse interpretiert werden. Zunächst wird ein Begriff von Solidarität als Form sozialer Beziehungen vorgestellt. Solidarische Beziehungen verleihen einem Kollektiv erweiterte Handlungsfähigkeit (2.1). Auf dieser Grundlage kann dann Kollegialität als Form der Solidarität beschrieben werden, die den Arbeitenden ermöglicht, den Arbeitsprozess gemeinsam zu gestalten. Handlungstheoretisch analysierbar wird Kollegialität als reziproke Austauschbeziehung, die in interaktiven Praktiken hergestellt und reproduziert wird (2.2).

\subsection{Solidarität und kollektive Handlungsfähigkeit}

Das Phänomen der Kollegialität wird hier begrifflich als eine Form von Solidarität gefasst, die auf kooperativer Arbeit im Betrieb basiert. Der Solidaritätsbegriff ist allerdings nicht leicht zu definieren. Sozialwissenschaftliche Debatten zeigen ein breites Spektrum an Bedeutungen (Hondrich \& Koch-Arzberger, 1992; Bayertz, 1998; Bierhoff \& Küpper, 1999; Zoll, 2000; Billmann \& Held, 2013; Laitinen \& Pessi, 2014), auf die an dieser Stelle nicht weiter eingegangen werden kann. Hier soll mit einem sozialphilosophischen Begriff von Solidarität gearbeitet werden, wie er insbesondere von Rahel Jaeggi im Anschluss an die kritische Theorie entwickelt wurde.

Ausgangspunkt ist zunächst, dass Arbeit nicht nur ein Mittel zur Existenzsicherung darstellt, sondern auch Bedürfnisse der Anerkennung und Selbstverwirklichung erfüllt. In der Arbeit nimmt das Individuum teil an der Herstellung gesellschaftlicher Güter und Dienstleistungen, vermittelt über die Arbeit erhält es die Möglichkeit, auch selbst davon zu profitieren. In der Zusammenarbeit unterschiedlicher Individuen entsteht zugleich eine Moral des Miteinanders (Honneth, 2008; Jaeggi \& Kübler, 2014; Jaeggi, 2017a).

Solidarität ist eine ,symmetrisch-reziproke und nicht-instrumentelle Beziehung des Füreinander-Einstehens auf der Grundlage geteilter Herausforderungen, Erfahrungen oder Projekte“ (Jaeggi \& Celikates, 2017, S. 39). Der Begriff umfasst sowohl faktisch bestehende soziale Verbindungen wie auch normative Erwartungen des Füreinander-Einstehens. Solidarität beruht nicht auf einer irgendwie gearteten gemeinschaftlichen Identität oder einer ursprünglichen Verbundenheit, sondern realisiert sich erst in kooperativer Praxis (ebd., S. 38-39). Solidarität ist nicht schicksalhaft, sie ist eine freie Art der sozialen Bindung (Hondrich \& Koch-Arzberger, 1992, S. 15).

Im Bewusstsein, in der Gesellschaft aufeinander angewiesen und miteinander verbunden zu sein, gehen Menschen Beziehungen ein, um Ziele zu erreichen, deren Realisierung 
nur gemeinsam denkbar ist. „Der Grund für die Solidarität ist die Überzeugung, dass Erfolg und Wohlergehen der Anderen für die Entfaltung derjenigen Projekte wichtig sind, mit denen ich mich selbst identifiziere“ (Jaeggi, 2017b, S. 93). Die Tatsache, auf andere angewiesen zu sein, bewirkt für sich noch kein solidarisches Denken und Handeln. Solidarität entsteht erst in der Realisierung der Gemeinsamkeit, im doppelten Sinn des kognitiven Begreifens und des praktischen Verwirklichens. „Solidarität verweist also auf die Fähigkeit, sich aktiv und positiv in Verbindung zu setzen und die gesellschaftlichen Bindungen und wechselseitigen Abhängigkeiten zu formen, in die die*der Einzelne faktisch immer schon eingebunden ist" (ebd., S. 98).

Damit ist zugleich gesagt, dass das solidarische „Wir“, die Gruppe, deren Mitglieder sich miteinander verbinden, nicht fixiert ist, sondern ebenfalls in der Praxis konstituiert wird. Das „Wir“ ist partikular, es kann eingeschränkt und ausgedehnt werden im Hinblick auf verschiedene Interdependenzen und Kooperationszusammenhänge (ebd., S. 97). Wie weit Solidarisierung reicht, hängt davon $a b$, inwieweit wechselseitige Verflechtungen des Handelns erkannt und Verbindungen bewusst gestaltet werden. Unsolidarisches Handeln ist ein Ausdruck der Unfähigkeit oder des Unwillens, Verbindungen zu anderen herzustellen, obwohl die eigene Lage mit der ihren gesellschaftlich verknüpft ist (ebd., S. 100-101).

Je weiter die Bande der Solidarität geknüpft werden können, desto größer wird die Chance, gesellschaftliche Verhältnisse auch in weiterem Rahmen zu verändern. Solidarisierungsprozesse sind „Prozesse der ,Ermächtigung “, die es erlauben, Situationen des gemeinsamen Lebens aktiv zu gestalten, denen man sonst passiv unterworfen wäre. Das bedeutet eine Erweiterung kollektiver Handlungsfähigkeit, die gleichzeitig die Möglichkeit der Individuen befördert, ihr eigenes Leben zu gestalten“ (Jaeggi \& Celikates, 2017, S. 40). Solidarität überschreitet die Alternative zwischen egoistischen und altruistischen Beweggründen, indem sie das eigene Schicksal in dem der anderen erkennt und darauf abzielt, gemeinsam die Verfügung über die Bedingungen des Handelns aller auszuweiten (Jaeggi, 2017b, S. 93).

Die Kritische Psychologie (Holzkamp, 1983, S. 356-415; vgl. Markard, 2005, S. 180221 und Süß, 2015, S. 73-110) unterscheidet zwischen restriktiver und verallgemeinerter Handlungsfähigkeit. In der kapitalistischen Gesellschaft ist das Individuum in sachzwangartig wirkende Herrschaftsverhältnisse eingebunden. Das Individuum hat die Möglichkeit, sich mit den restriktiven gesellschaftlichen Bedingungen abzufinden und die gegebenen Möglichkeiten für sich auszunutzen. Es kann aber auch versuchen, seine Handlungsmöglichkeiten dadurch zu erweitern, dass es die gesellschaftlichen Bedingungen verändert (Holzkamp, 1983, S. 368-370).

Letzteres impliziert aus zwei Gründen eine Kooperation mit anderen: Zum einen ist eine erweiterte Verfügung über die eigenen Lebensbedingungen in einer arbeitsteiligen Gesellschaft nur möglich in Form einer Teilhabe an der kollektiven Verfügung über die Mittel zur Gestaltung gesellschaftlicher Prozesse (ebd., S. 363). Zum anderen bedeutet der Versuch, die Bedingungen zu verändern, immer auch eine Gefährdung der Existenz, weil die bis dahin erreichte Handlungsfähigkeit aufgegeben wird und nicht sicher ist, ob sich die angestrebte Erweiterung realisieren lässt. Wer sich beispielsweise an einem Streik beteiligt, muss damit rechnen, vom Arbeitgeber sanktioniert zu werden und danach schlechter gestellt zu werden als vorher. Nur im Zusammenschluss mit anderen kann eine überindividu- 
elle Gegenmacht etabliert werden, die eine Gefährdung der Existenz aufheben kann (ebd., S. 371-373). So vereinbaren Gewerkschaften in der Regel mit jedem Tarifabschluss auch ein Verbot für den Arbeitgeber, die Streikenden zu maßregeln.

Im Gegensatz dazu ist restriktive Handlungsfähigkeit gekennzeichnet durch eine Anerkennung der Machtverhältnisse und das Gegeneinander unterschiedlicher Partialinteressen. Im Rahmen gegebener Verteilungsspielräume sind die eigenen Interessen durch die Interessen anderer eingeschränkt, das Individuum erreicht Verfügung über seine Lebensbedingungen nur auf Kosten anderer. Zusammenschlüsse sind unter der Prämisse der Anerkennung gegebener Verhältnisse für das Individuum nur funktional, wenn dadurch die Fähigkeit zur Durchsetzung gemeinsamer Partialinteressen gegen andere Partialinteressen gestärkt werden kann. Die eigentliche Macht bleibt dabei unangetastet (ebd., S. 374). Die Beziehungen in einer solchen Interessenkoalition sind charakterisiert durch gegenseitige Instrumentalisierung. Wenn eine Person andere instrumentalisiert, muss sie damit rechnen, dass die oder der andere genauso handelt. „Indem ich ihn von mir isoliere, isoliert er mich von sich. Damit bin ich, im Versuch, mich durch die Kontrolle anderer abzusichern, immer mehr auf mich selbst zurückgeworfen, also immer ohnmächtiger den von mir unverfügbaren Lebensbedingungen ausgeliefert“" (ebd., S. 377).

\subsection{Kollegialität, Reziprozität und Praktiken des Tauschens}

Solidarische Beziehungen, wie sie in der Praxis der betrieblichen Zusammenarbeit interaktiv hergestellt werden, sollen im Folgenden als „Kollegialität“ bezeichnet werden. Entgegen dem allgemeinen Sprachgebrauch, jede und jeden im gleichen Betrieb als Kollegin oder Kollegen zu bezeichnen (mit der paradoxen Konsequenz, dass es dann auch „unkollegiale“ Kolleginnen und Kollegen gibt), soll hier mit dem Begriff der Kollegialität eine intersubjektive Beziehung bezeichnet werden, die im Arbeitszusammenhang aktiv von den beteiligten Personen hergestellt wird, um ihre Bindungen und wechselseitigen Abhängigkeiten zu formen, in welche die bzw. der Einzelne faktisch schon eingebunden ist.

Wie „Solidarität“ hat auch der Begriff der Kollegialität ein normatives Moment, er wird von den Beschäftigten wertend verwendet für eine Beziehung des FüreinanderEinstehens. Die Gemeinsamkeit der Arbeitenden besteht nicht nur darin, dass sie sich bei der Arbeit in gleicher Situation befinden, sondern vor allem darin, dass sie aktiv einen gemeinsamen Bezug zu ihr herstellen und gemeinsam handeln (Bachmann, 2014, S. 41-43). „Das Miteinander ist nicht nur ein Aufeinander-Bezogensein, sondern auch gemeinsames Tun, affektives Einschwingen, Nebeneinander und Zusammengehören. Aus dem Miteinander heraus entwickeln sich Beziehungen und Bindungen, Formen des Gebens, Nehmens und Teilens, Kreise des geteilten Wissens und die Freiheiten und Einschränkungen gemeinsamer Selbstentwürfe“ (ebd., S. 414). Die Bezüge gestalten sich in der Regel partikular in Dyaden, Gruppen und anderen Zusammenschlüssen, können aber auch übergreifend auf die ganze Belegschaft und darüber hinaus gestaltet werden (ebd.).

Anders als Freunde kommen Kolleginnen und Kollegen nicht freiwillig zusammen. Sie treffen bei der Arbeit aufeinander, es gibt ein funktionales Erfordernis zur Zusammenarbeit, auch wenn man sich persönlich vielleicht nicht sympathisch ist. Auf der anderen Seite impliziert Kollegialität ein Moment der gefühlsmäßigen, moralischen Verbundenheit und des gegenseitigen Einvernehmens, das über das sachlich-funktionale Verhältnis hinausgeht. 
Kollegialität beinhaltet ein Spannungsverhältnis zwischen „Mensch-Sein“ und „LeistungBringen“ (Hürtgen, 2013). Im Arbeitsalltag muss eine Balance gefunden werden zwischen den subjektiv-menschlichen Aspekten der Zusammenarbeit und den Leistungsanforderungen des Betriebes. Letztere setzen die Rahmenbedingungen. Wenn die Leistungsanforderungen zu hoch und die (Zeit-)Ressourcen zu gering sind, um Beziehungen zu pflegen und Unterstützung zu leisten, kann dies die Entwicklung von Kollegialität ebenso behindern wie eine Unternehmenskultur, die auf Wettbewerb und Konkurrenz angelegt ist (Daser, 2011).

Kollegialität beruht auf Gegenseitigkeit. Wer Unterstützung von anderen erwartet, muss sich hilfsbereit zeigen, wenn diese ihrerseits Unterstützung benötigen. Der interaktive Prozess zur Herstellung von Kollegialität lässt sich interpretieren als reziproker Austausch von sachlichen Arbeitsleistungen sowie persönlicher Hilfe und Unterstützung. Reziprozität ist eine Form der Interaktion von Akteurinnen und Akteuren, die ihr Handeln aufeinander abstimmen und an gegenseitigen Erwartungen ausrichten (Hillebrandt, 2009, S. 152).

In Theorien zur Reziprozität wird unterschieden zwischen den Formen des Waren- und des Gabentausches (Pulcini, 2004; Komter, 2005; Caillé, 2008; Hénaff, 2009). Mit dem Begriff der Gabe werden alle Formen der schenkenden Zuwendung an Mit-Akteure bezeichnet. Die Gabe kann auch aus Gesten, Hilfeleistungen und Diensten bestehen (Hillebrandt, 2009, S. 136). Waren- und Gabentausch folgen zwei verschiedenen Logiken, die sich in der Praxis durchkreuzen, befördern und behindern. Wer eine Ware verkauft, will dafür Geld haben oder eine andere Ware von gleichem Wert. Der Käufer will einen Gegenstand erwerben und muss dafür einen angemessenen Preis zahlen. Bei der Gabe dagegen geht es nicht um den Gegenstand selbst, sie ist vielmehr ein Symbol für die soziale Beziehung. Wer jemand anderem etwas schenkt, tut dies aus dem Antrieb heraus, eine Beziehung zum Gegenüber zu beginnen oder fortzusetzen. Die Gabe fordert keine gleichwertige Gegengabe, man gibt vielmehr, um sich mit anderen zu verbinden, im Bewusstsein, als Individuum nur in sozialen Beziehungen bestehen zu können. „Daher könnte man sagen, die Gabe sei ein Zeichen für das Eingedenken der eigenen Abhängigkeit und eine emotionale Manifestation, ein aktives Zeugnis des eigenen Verlangens nach Bindung“ (Pulcini, 2004, S. 193).

Beide Formen des Tausches finden sich auch in den Arbeitsbeziehungen (Hillebrandt, 2009, S. 172-176; vgl. Hénaff, 2009, S. 574-577). Beschäftigte erwarten für ihre Leistung eine Gegenleistung, nicht nur vom Arbeitgeber, sondern auch von denen, die mit ihnen zusammenarbeiten. Leistungen werden verglichen und gegeneinander abgewogen. Wer jemand anderem Arbeit abnimmt, erwartet, dass diese bzw. dieser bei Gelegenheit gleiches tut. Neben dieser berechnenden Logik von Leistung und Gegenleistung kommt jedoch immer auch die Logik des Gabentausches zum Tragen, die auf soziale Bindungen zielt aus der Erkenntnis heraus, gemeinsame Ziele zu verfolgen und aufeinander angewiesen zu sein. Der Arbeitsprozess würde nicht funktionieren, wäre er nur von egoistischen Nutzenerwägungen und nicht auch von solidarischen Motiven geleitet, die dafür sorgen, dass auf spontane, nicht berechnende Weise Hilfe und Unterstützung geleistet wird (Caillé, 2008, S. 95).

Die Entwicklung von Kollegialität hat eine reale Grundlage im Arbeitsprozess, der ein eigenständiges Zusammenwirken der Arbeitenden und die Gestaltung ihres Leistungsaustausches wie auch gegenseitige Unterstützung und Hilfestellung erfordert. „Wenn Menschen kooperieren, setzen sie sich eines gemeinsamen Zwecks wegen mit ihren Handlungen 
wechselseitig ins Verhältnis zueinander.“ (Projektgruppe Automation und Qualifikation, 1986, S. 82) Zwar sind die Arbeitsprozesse vorstrukturiert durch Technik, Arbeitsteilung, Regeln und Anweisungen, können aber niemals ganz formalisiert werden, immer stellt sich die Anforderung an die Individuen, die eigene Tätigkeit in Bezug zu setzen zu den Tätigkeiten der anderen. Kooperation hat eine vermittelnde Funktion (ebd., S. 80).

Kollegiale Kooperation wird zugleich begrenzt und behindert durch Motive der Konkurrenz, die bedingt sind durch die Gesetze des Arbeitsmarkts und die Vormachtstellung des Arbeitgebers, die sich in der betrieblichen Hierarchie manifestiert. Welche Handlungsweisen sich im Arbeitsprozess im Widerstreit von Herrschaft und Autonomie, Solidarität und Konkurrenz entwickeln, wird von strukturellen Rahmenbedingungen aus Hierarchie, Beschäftigungsverhältnissen oder Entgeltgestaltung zwar vorgeprägt, ihre konkrete Ausformung erhalten sie jedoch erst in der täglichen Praxis. Die empirischen Kooperationsbeziehungen sind auch als „Verarbeitungen von Widerspruchskonstellationen“ zwischen den Anforderungen der Zusammenarbeit und den Herrschafts- und Konkurrenzverhältnissen zu lesen (ebd., S. 84).

Praktiken werden in der Praxistheorie als ,elementare Operationen, also als Ereignisse jeder Sozialität“ gefasst (Hillebrandt, 2009, S. 88), als „eine Kombination aus Sprechakten, körperlichen Bewegungen (...) und einer durch Assoziation zwischen sozialisierten Körpern und materiellen Artefakten ermöglichten Handhabe der Dinge“ (ebd., S. 87). Sie können sich zu Praxisformen verketten, in denen die Entstehung von Praktiken an einem bestimmten Ort im sozialen Raum auf Dauer gestellt ist und relativ sicher erwartet werden kann (ebd., S. 84). Praktiken lassen sich nicht erklären als Erscheinungsformen objektivierter sozialer Strukturen oder ableiten aus vorab festgelegten Regeln. Sie sind „Konstitutionsereignisse sozialer Praxisformen, deren Eigenlogik jenseits vorab theoretisch festgelegter Regelsysteme analysiert werden muss“ (ebd., S. 83). Praktiken des Tausches sind in diesem Zusammenhang paradigmatische Beispiele für soziale Praktiken, weil der Tausch nur zwischen mindestens zwei Akteurinnen bzw. Akteuren stattfinden kann (ebd., S. 88).

Durch Institutionalisierung von Praktiken des Tauschens berechenbarer Leistungen und spontaner Unterstützung entsteht in der alläglichen Zusammenarbeit eine - in weiten Teilen implizite - Ordnung der Reziprozitäten, in der das Geben und Nehmen geregelt ist (Kock \& Kutzner, 2014, S. 289-310; vgl. Hillebrandt, 2009, S. 204). Die Arbeitenden eignen sich die etablierten Regeln an, sie wissen, was zu tun ist, um ihre Arbeit auszuführen. Indem sie handeln, reproduzieren sie die Ordnung des Gebens und Nehmens. Sie tun dies jedoch nicht unreflektiert, sondern im Bewusstsein faktischer Notwendigkeit einerseits, mit moralischen Sollensansprüchen andererseits.

\section{Praktiken zur Herstellung von Kollegialität - empirische Analysen}

Die in diesem Abschnitt analysierten Interviewpassagen stammen aus einer in den Jahren 2011-2013 durchgeführten Untersuchung zum Thema „Betriebsklima, Reziprozität und gute Arbeit“ (Kock \& Kutzner, 2014). Mit qualitativen Methoden der Sozialforschung wurden Praktiken des reziproken Austausches und Formen ihrer Verfestigung in Gewissheiten, 
Routinen und Strukturen untersucht als Determinanten der Zusammenarbeit, deren Qualität Beschäftigte mit der Metapher „Betriebsklima“ beschreiben. Im Mittelpunkt stand dabei die Sicht der Beschäftigten und ihrer Vorgesetzten, die durch Interaktionen und Kommunikationen den Betrieb als Lebenswelt konstruieren.

Die empirische Basis des Projekts bilden sechs Fallstudien in zwei Stadtverwaltungen (inklusive Kindertagesstätte und Bauhof), zwei Industriebetrieben (Nahrungsmittelherstellung und Elektroindustrie) und zwei Dienstleistungsbetrieben (Großhandel und Weiterbildungsbranche). Hier wurden zunächst jeweils die strukturellen Rahmenbedingungen mit Hilfe von Dokumenten und Interviews mit Expertinnen und Experten erhoben. Hauptteil der Untersuchung waren insgesamt 97 narrative Interviews mit Vorgesetzten und Beschäftigten sowie 24 Gruppendiskussionen vorwiegend mit realen Gruppen aus Beschäftigten, die im Arbeitsalltag interagieren und kooperieren. Das Material wurde mit Hilfe der dokumentarischen Methode ausgewertet und zu zwölf zentralen Kategorien verdichtet. In Form eines Glossars wurden die Ergebnisse so aufbereitet, dass sie sowohl für die Sozialwissenschaft als auch für die betriebliche Praxis les- und handhabbar sind.

Aus dem Interviewmaterial dieser Studie werden auf der oben entwickelten theoretischen Grundlage im Folgenden einige Praktiken aus dem Arbeitsalltag analysiert, in denen Kollegialität hergestellt wird. Insofern die Kooperation der Arbeitenden nicht durch Regeln oder Anordnungen vorgeschrieben ist, geht es für sie zunächst darum, Einvernehmen zu erzielen über das gemeinsame Ziel und die Wege dorthin. Im Rahmen der Zusammenarbeit als soziale Beziehung kommt es zweitens darauf an, dass die Kolleginnen und Kollegen ein persönliches Verständnis füreinander entwickeln. Die Schnittstellen der Teilarbeiten, so wird im dritten Abschnitt argumentiert, überbrücken die Arbeitenden dadurch, dass sie einander entgegenkommen. Im vierten Abschnitt schließlich werden Praktiken spontaner gegenseitiger Hilfeleistungen behandelt, die notwendig für die Arbeit sind, zugleich aber auch soziale Bindungen zwischen den Beschäftigten stiften.

Gegenseitige Abhängigkeiten, wie sie im Rahmen arbeitsteiliger Organisationen entstehen, sind Herausforderung zur Kooperation, bergen zugleich aber auch das Potenzial für Interessenkonflikte. Wenn zwei oder mehrere Parteien versuchen, unterschiedliche, nicht vereinbare Handlungspläne zu verwirklichen, entstehen Spannungssituationen, die entweder durch Abstimmung der Handlungen im allgemeinen Interesse aufgelöst oder aber in konflikthaften Auseinandersetzungen entschieden werden, sei es indem eine Seite sich durchsetzt, sei es dass ein Kompromiss geschlossen wird (Spieß, 2007, S. 339-341). Interviewpassagen zu gelungener Kooperation werden deshalb im Folgenden konfrontiert mit Aussagen über konflikthafte Situationen, um zu zeigen, dass kollegiale Praktiken sich nicht spontan durchsetzen, sondern gegen widerstreitendende Tendenzen realisiert werden.

\subsection{Einvernehmen herstellen}

Voraussetzung für den Erfolg kooperativer Arbeit ist ein gewisses Einvernehmen darüber, was der Sinn des Ganzen ist und wer dazu welchen Beitrag leisten soll. Die Arbeitsaufgabe ist zwar in der Regel von der Organisation vorgegeben, muss aber - vor allem dort, wo Vorgesetzte nicht steuernd eingreifen - subjektiv und im täglichen Miteinander angeeignet und umgesetzt werden. Im Folgenden wird gezeigt, dass Einvernehmen vorwiegend diskur- 
siv hergestellt wird. Es wird auch deutlich, dass die Breite des Diskurses variieren kann, dass unter Machtgesichtspunkten auch Ausschließungen stattfinden.

Erzieherinnen in einer Kindertagesstätte verwendeten in einer Gruppendiskussion für einvernehmliches Handeln die Metapher ,an einem Strang ziehen“:

„Es ist nicht für jeden dasselbe Ziel das richtige. Das ist auch ganz gesund eigentlich, wenn nicht jeder dieselbe Ansicht hat. Das ist immer meine Meinung. Da hat jeder seine eigene Ansicht, und das muss man aber in die Reihe kriegen dann.“ „Der Meinung bin ich auch, das verstehe ich auch unter , an einem Strang ziehen'. Auch wenn man gegensätzliche Meinungen hat, aber man kann diese unterschiedlichen Meinungen ausdiskutieren und letztendlich doch auf einen Nenner kommen. Also den Mittelweg vielleicht finden oder so. Das ist dann , an einem Strang ziehen“.”

Das Selbstverständnis der Personengruppe als kollegiales Team entwickelt sich über den kontinuierlichen Prozess einer bewusst geführten Auseinandersetzung. Die Erzieherinnen stellen Einvernehmen über ihre pädagogischen Aufgaben vorwiegend diskursiv her, indem sie Argumente und Meinungen austauschen.

Voraussetzung ihrer guten Zusammenarbeit, sagten uns Mitarbeiterinnen und Mitarbeiter in einer Abteilung einer Stadtverwaltung, sei, dass sie voneinander wissen, was die bzw. der andere im Sachgebiet jeweils tut, und dass sie sich absprechen, wer welchen Beitrag zum gemeinsamen Ziel erbringen soll. Die Zusammenarbeit funktioniere gut,

„weil wir sehen, was der andere macht. Wir gucken über unseren eigenen Schreibtisch. Wenn ich sehe, was die Frau X macht, ich sehe, was Herr Y macht. Ich sehe, was die Frau Z macht. Und genauso ist es anders herum, sie weiß, was ich mach'. Sie weiß, was er macht. Sie weiß, was sie macht. Dadurch, dass halt jeder dem anderen hilft, hat er ja auch Einblick in die Aufgabe.“

Einvernehmen wird hergestellt durch Interesse an der Arbeit der anderen, Einordnung der einzelnen Beiträge in das Ganze und situativen reziproken Austausch von Arbeits- und Unterstützungsleistungen, über die täglich verhandelt wird.

Was im Team gut funktioniert, kann jedoch an den Grenzen der eigenen Abteilung Halt machen. Für die Arbeit der Beschäftigten in anderen Ämtern der Stadtverwaltung entwickeln manche Befragte nur wenig Verständnis. Nach einer Klage über die eigene Arbeitsbelastung hieß es in einer Gruppendiskussion:

„Was machen die eigentlich da (im Amt X), die haben da zehn Mann, und was kommt denn da eigentlich rüber so am Monatsende? Wir können's auch nicht richtig einschätzen.“ „Ne, aber es ist schon sehr verwunderlich. Es gibt wirklich Schreibtische im Rathaus, die sind hochglanzpoliert und kein Staubkörnchen, wenn man ins Zimmer kommt, da liegt nichts zum Arbeiten drauf.“

Es fehlt hier offenbar an Wissen darüber, aber auch an Interesse daran, welchen Beitrag andere leisten und wie die eigene Arbeit mit den Tätigkeiten der anderen in Verbindung steht. Die Kollegialität bleibt auf den eigenen Bereich des Sachgebiets beschränkt. Verbindungen zu anderen Ämtern und Sachgebieten werden nicht hergestellt. Damit bleibt die Handlungsfähigkeit der Kolleginnen und Kollegen begrenzt. Sie sind beispielsweise nicht in der Lage zu beeinflussen, wie viel Personal sie selbst und andere Abteilungen zugeteilt bekommen. Wüssten sie mehr über Inhalt und Umfang der Tätigkeiten anderer Ämter, könnten sie unter Umständen direkt fragen, ob jemand von dort, wo gerade nicht so viel zu tun ist, zeitweise bei ihnen aushelfen könnte, um ihre Arbeitsbelastung zu reduzieren.

Eine Verwaltungsangestellte bei einem Weiterbildungsträger schilderte im Interview, es gebe immer wieder Auseinandersetzungen zwischen den Beschäftigten der Buchhaltung 
und dem pädagogischen Personal. Bei finanziellen Fragen muss die Buchhaltung exakt arbeiten, sie kann nicht Geld auszahlen ohne Belege oder Angabe der Kostenstelle. Damit stoßen sie oft auf Unverständnis, das sich in abschätzigen Bemerkungen über ihre Arbeit insgesamt widerspiegelt:

„Wir hatten mal einen Fall gehabt, da kam ein neuer Mitarbeiter, also jetzt nicht bei uns (sondern aus dem pädagogischen Bereich), und der wurde dann hier unter anderem auch vorgestellt, dann hieß es gleich: ,Können Sie gleich vergessen, das ist die Buchhaltung. 'Ja und wenn Sie das dann so zufällig mitkriegen, so nach dem Motto - was sind wir denn hier? (...) Ja gut, der Schwerpunkt ist nun mal hier in der Firma - sind nun mal die Sozialpädagogen und deren Tätigkeit, aber ohne Buchhaltung geht's auch nicht.“"

In der Produktionsabteilung eines Industriebetriebs fanden wir ein Beispiel dafür, wie mögliches Einvernehmen über die Arbeit zwischen Beschäftigtengruppen, deren Arbeit faktisch eng zusammenhängt, geradezu verhindert wird. Hier sind einige Männer aus dem Kreis der Maschinenbedienerinnen und -bediener („Operators“) zu Mechanikern befördert worden mit der Aufgabe, die Maschinen zu warten und zu reparieren. Frauen werden von den Vorgesetzten für diese Aufstiegsposition systematisch nicht berücksichtigt, obwohl einige von ihnen über mindestens die gleichen langjährigen Erfahrungen verfügen wie die Männer. In der Regel arbeiten Mechaniker und Maschinenbedienerinnen zusammen. Je nach Erfahrung beheben die Bedienerinnen manche Störungen selbst, für andere rufen sie die Mechaniker. Wenn es jedoch etwas zu besprechen gibt, wird diese Interaktion faktisch abgebrochen, die Mechaniker ziehen sich in einen gesonderten Raum zurück. Danach erst informieren sie die anderen und ordnen an, was geschehen soll.

Die Betriebsratsvorsitzende, die selbst als Maschinenbedienerin arbeitet, ist der Ansicht, durch diese Verweigerung des Informationsaustausches werde die Zusammenarbeit erheblich gestört, weil an den Maschinen das notwendige Wissen fehle:

„Zum Beispiel jetzt Schichtübergabe. Überlegen Sie mal: Schichtübergabe, der Operator muss doch genau
das gleiche wissen wie der Mechaniker - welche Maschinen Probleme haben, welche Maschinen nicht lau-
fen, was für Störungen da sind. Nein, die gehen raus und besprechen das nicht vor den Operatern. Die gehen
raus und führen die Gespräche. Sowas kenne ich ja gar nicht von anderen Firmen. Es ist nicht möglich. Du
musst als Operator genau wissen, was abläuft. Ich komm' in die Linie, ich weiß gar nicht, was los ist. Oder
die Maschine wird gestoppt, rote Lampe, das ist mein Bereich, mein Name steht da, ich weiß gar nicht, was
da los ist. Da wird nicht kommuniziert. Da wird immer so - der Operator ist dumm, der braucht nichts wis-
sen.“

Die Mechaniker verfolgen offenbar ihre eigenen Ziele, ihr Verhalten gegenüber den Maschinenbedienerinnen zielt darauf, Wissen zu monopolisieren, sich selbst dadurch eine überlegene Stellung zu verschaffen und letztlich auch ihre bessere Bezahlung zu rechtfertigen. Kollegialität praktizieren sie nur unter sich, gegenüber den Maschinenbedienerinnen dient ihr Zusammenhalt als Mittel der Ausgrenzung. Dieses Machtverhältnis ist hier eindeutig geschlechterbezogen strukturiert. Diese Männer beanspruchen „das Sagen“, die Frauen sollen das Gesagte ausführen. Im Endeffekt wird die Zusammenarbeit beeinträchtigt, es entsteht Frustration und Wut auf Seiten der bevormundeten Maschinenbedienerinnen.

Aber die vorteilhafte Situation der Mechaniker bleibt prekär, weil sie aus der Gunst der Vorgesetzten und der Zugehörigkeit zu einer Interessengruppe resultiert, worauf sich im Konfliktfall niemand berufen könnte. Wer sich auf wen verlassen kann, ist mit davon ab- 
hängig, in welcher Interessenkonstellation man selbst und der bzw. die andere sich befinden. Kollegialität wird zu einer Frage der Taktik.

Die zitierten Interviewpassagen zeigen, dass Kollegialität einerseits eine Reaktion auf die Erfordernisse des jeweiligen Arbeitsprozesses darstellt. Nur durch reziproken Austausch von Leistungen, Informationen und Hilfestellungen ist die einzelne Arbeitsaufgabe zu bewältigen und wird zum Teil des Ganzen. Andererseits handelt es sich um eine aktive Auseinandersetzung mit den Gegebenheiten. Die Arbeitenden definieren ihre jeweiligen Tätigkeiten auf unterschiedliche Weise als gemeinsame Aufgabe und stellen auf diskursive Weise Einvernehmen darüber her, was zu tun ist.

Zugleich wird deutlich, dass Grenzen der Kollegialität gezogen werden, indem die Arbeitenden sich auf ihren unmittelbaren Arbeitsbereich beschränken und nicht versuchen, ihre Arbeit in den Gesamtzusammenhang einzuordnen. Das letzte Beispiel aus einem Industriebetrieb zeigt, dass Einvernehmen bewusst partikular hergestellt werden kann. Die Mechaniker verfolgen ihr eigenes Ziel. Sie monopolisieren ihr Wissen und verweigern den Informationsaustausch. Um ihren Status abzusichern und in eine überlegene Position zu gelangen, handeln sie kollegial nur unter sich, aber bewusst unkollegial gegenüber den Maschinenbedienerinnen.

\subsection{Verständnis entwickeln}

Weil Arbeit immer auch von individueller Motivation und persönlichem Engagement geprägt ist, erfordert Kooperation unterschiedlicher Individuen ein gegenseitiges persönliches Verständnis füreinander, das mehr umfasst als das Wissen um die Fähigkeiten und funktionalen Beiträge der/des jeweils anderen. Das Miteinander auf der sachlichen Ebene wird erst möglich durch die aktive Entwicklung einer persönlichen Umgangsweise. Eine Erzieherin verwandte dafür in einer Gruppendiskussion die treffende Metapher, man müsse sich ,aufeinander einstellen“.

Es sei wichtig, die individuelle Arbeitsweise der Kolleginnen und Kollegen zu kennen, hörten wir in einem Industriebetrieb der Lebensmittelbranche. Ein Befragter am Ende der Backstraße beispielsweise weiß oft schon im Vorhinein, worauf er achten muss, wenn ein bestimmter Kollege weiter vorne arbeitet, denn dieser Kollege hat seine eigene Art zu arbeiten - aus Sicht des Befragten arbeitet er vor allem langsamer als er selbst. Wenn die Zusammenarbeit funktionieren soll, müssen die anderen dies verstehen und ihr eigenes Handeln darauf einstellen.

„Wir sehen schon, wenn wir kommen, wir gucken, wer da steht. ,Schon wieder der Herr X, mein Gott. 'Wir sind bei uns in unserer Gruppe drei Bäcker. Und wir sehen: ,Ah, das war's. ' Da bereiten wir uns schon mal ein bisschen vor.“

Die Kollegen haben im Laufe der Zeit gelernt, mit dieser Person auf bestimmte Weise zu kooperieren, damit die Produktion reibungslos läuft. Im Bewusstsein, dass auch sie selbst ihren eigenen „Arbeitsstil“ entwickelt haben, nehmen sie solche in der Person liegenden Besonderheiten durchaus als Handlungsspielräume und Herausforderungen für persönliches Engagement wahr. Allerdings werde dieser Zusammenhang - so jedenfalls die Meinung der befragten Beschäftigten - von Vorgesetzten missachtet. Es werde zu häufig gewechselt an den Anlagen, so dass es kaum möglich sei, Erfahrungen miteinander zu sammeln, hieß es in einer Gruppendiskussion. 
Wer Kenntnis hat von persönlichen Eigenheiten, Einstellungen und Stimmungen der anderen, kann besser mit ihnen umgehen. Manche Handlungsweisen werden verständlich, mögliche Konflikte können vermieden werden. Eine Mitarbeiterin einer Stadtverwaltung betonte im Interview, wie wichtig es ihr sei, dass sie sich in ihrem Sachgebiet gut miteinander verstehen:

„Es gibt ja manchmal Situationen, wo man denkt: Was habe ich jetzt schon wieder gemacht, was habe ich jetzt falsch gemacht? Warum reagiert mein Gegenüber so oder der Kollege so? Und da ist es dann halt schön zu wissen, dass man auf den zugehen kann und kann sagen: Hör mal zu, ist irgendwas? Hat's jetzt mit mir zu tun? Ich sage mal, man will mit den Kollegen nicht verheiratet sein, man muss auch nicht alles wissen, aber es erleichtert das Zusammensein, sag' ich mal.“

Von Vorgesetzten oftmals misstrauisch beobachtete „Privatgespräche“ während der Arbeit gewinnen so eine produktive Funktion. Weil sich die Kolleginnen und Kollegen als Personen näher kennen, wird der Umgang miteinander leichter. Missverständnisse werden vermieden, Reaktionen der anderen können abgeschätzt und berücksichtigt werden. Aus solchem gegenseitigen Verständnis erwächst ein Zusammenhalt, in dem die/der Einzelne Wertschätzung verspürt und sich aufgehoben fühlt. Wenn jemand ,einen schlechten Tag“ hat, nehmen die anderen Rücksicht, erledigen einiges mit, was nicht zu ihren eigentlichen Aufgaben gehört. Gegenüber Vorgesetzten treten Kolleginnen und Kollegen füreinander ein, machen vielleicht sogar deutlich, dass sie Sanktionen gegen einzelne als gegen das Kollektiv gerichtet empfinden und entsprechend kollektiv reagieren werden.

Allerdings, so wurde uns berichtet, kann das Gefühl entstehen, dass einzelne das Verständnis der anderen zu ihrem persönlichen Vorteil ausnutzen. So kam in einer Gruppendiskussion mit Beschäftigten aus einer Stadtverwaltung zur Sprache, dass einige Kolleginnen und Kollegen auffallend häufig krank sind. Sie bekommen den Ärger der anderen zu spüren:

„Es wird ziemlich viel gelästert. Also es wird gelästert, ja auch wenn mal - na ja einmal eine Krankheit ist wegen Magen-Darm, nächste Woche dann eine Krankheit ist wegen Grippe, wenn dann wieder ein Ausfall ist - wie auch immer - wegen Pollen(allergie), dann wird gelästert. (...) Aber ich glaub', das ist auch ein Stück weit menschlich, und dass die Zeit sich so verändert, dass man gerne mal auch einen Stich loslässt und eine ironische Bemerkung. Und ich glaub', wir sind hier alle mittlerweile so am Limit, dass man das ganz schlecht haben kann einfach und versucht, das zu vermeiden.“

Füreinander einzustehen bedeutet im kooperativen Arbeitsprozess immer auch, gemeinsam dafür zu sorgen, dass die geforderte Leistung erbracht wird. Wenn es an Personal fehlt und die Leistungsanforderungen sich dadurch erhöhen, verringert sich der Spielraum für gegenseitige Unterstützung. Kolleginnen und Kollegen fragen sich, ob die eigene Leistung noch in einem angemessenen Verhältnis zur Leistung der anderen steht. Das Verständnis für persönliche Probleme nimmt ab, berechnendes Verhalten nimmt zu. Um weiterhin kollegial miteinander umgehen zu können, wäre es notwendig, eine bessere Personalausstattung durchzusetzen, was allerdings auf einer höheren Ebene der Hierarchie entschieden wird.

Es gibt Grenzen für Privates, auch aus Sicht der Beschäftigten. Kollegialität ist nicht gleichbedeutend mit Freundschaft. Die Befragten unterschieden sehr wohl zwischen Arbeits- und Privatbereich. Man versteht sich gut, das sei förderlich für die Arbeit. Es sei aber auch eine gewisse Gelassenheit vonnöten, man dürfe nicht alles persönlich nehmen, erläuterten uns die Erzieherinnen einer Kindertagesstätte: 
„Das ist hier meine Arbeit. Das sind meine Kollegen, und hier geht es ums Arbeiten und um das Miteinander. Und das muss nicht unbedingt in meinem Privaten alles dem entsprechen. Das kann - das geht nicht. So was ginge nie. Und wenn man, denke ich mal, so ein bisschen, so ein bisschen mehr Gelassenheit da anbringt, dann kann man das auch mit ein bisschen mehr Gelassenheit insgesamt beurteilen.“

Die Interviewpassagen zeigen, dass Kollegialität eine persönliche Komponente hat, indem die Arbeitenden ein Verständnis füreinander als Personen entwickeln und sich dementsprechend ,,aufeinander einstellen“. Ob dies gelingt, ist von außen bzw. von Vorgesetzten - beispielsweise durch die Personalauswahl - nur begrenzt zu steuern. Im Wesentlichen bleibt es den betreffenden Beschäftigten überlassen, ob und wie sie ihr Miteinander gestalten. Wie weit sich jemand persönlich einbringt, was er bzw. sie über das Privatleben offenbart und umgekehrt von anderen wissen möchte, ist individuell verschieden und ergibt sich in der täglichen Interaktion. Im Unterschied zur Freundschaft hat Kollegialität eine sachliche Basis in der gemeinsamen Arbeitsaufgabe. Sachliche Gelassenheit kann die Arbeitswelt gerade attraktiv erscheinen lassen im Vergleich zu stärker gefühlsbetonten Liebes- und Freundschaftsbeziehungen zu Hause.

Die Herausforderung der Kollegialität besteht offenbar darin, Persönliches und Sachliches so miteinander zu verbinden, dass die gemeinsame Arbeit bewältigt wird und die beteiligten Personen Wertschätzung erfahren. Eine gewisse Kenntnis privater Lebensumstände der jeweils anderen fördert die sachliche Zusammenarbeit. Unter Bedingungen erhöhten Leistungsdrucks kann diese Balance in Gefahr geraten und berechnendes, konkurrentes Verhalten an die Stelle kollegialer Beziehungen treten. Diese Bedingungen zu gestalten, ist eine breitere Kollegialität vonnöten, die den Bereich der unmittelbaren Kooperation überschreitet und beispielsweise in Form einer Betriebsvereinbarung regelt, wie Personal zu bemessen ist.

\subsection{Entgegen kommen}

Die Teilarbeiten einer Gruppe, einer Abteilung oder auch eines Betriebes greifen nicht wie Zahnräder ineinander, sondern werden von den Arbeitenden aktiv und bewusst so gestaltet, dass sie sich zu einem sinnvollen Ganzen zusammenfügen. Insbesondere geht es dabei darum, nicht strikt an den Grenzen der eigenen Tätigkeit haltzumachen, sondern den Bezug auf andere Teilarbeiten mitzudenken und den Kolleginnen und Kollegen entgegenzuarbeiten. Auch unter diesem Blickwinkel ist das Funktionieren der arbeitsteiligen Produktion davon abhängig, dass die Arbeitenden mehr tun als formal von ihnen gefordert wird. Es entstehen kollegiale Beziehungen, die über den Funktionsbedarf hinaus ins Persönliche hineinreichen.

Eine Angestellte in der sog. Sollerfassung einer Stadtverwaltung, wo Belege und Rechnungen in die EDV eingegeben werden, muss immer wieder Kontakt aufnehmen zu Kolleginnen und Kollegen in anderen Abteilungen,

„weil ja so viele Daten für die Erfassung da sein müssen, so verschiedene Sachen: Betrag, Fälligkeit, sonst was. Dann manchmal fehlen die Sachen, oder von manchen Kollegen wird das auch nicht wirklich draufgeschrieben. Wir sagen denen das zwar: Bitte füllt das richtig aus. Aber irgendwie wird das auch nicht richtig gemacht."

Es ist nicht nur eine Frage korrekten Arbeitens, wie die Belege an die Sollerfassung weitergegeben werden, ob alles vollständig und korrekt ist, ob es rechtzeitig geschieht usw. Es 
geht darum, die Folgen des eigenen Handelns für die Tätigkeiten der anderen zu bedenken und ihnen so weit entgegenzuarbeiten, dass sie mit ihrer Tätigkeit reibungslos anschließen können. Die Befragte weiter:

\begin{abstract}
„Wir haben das auch öfters, wenn irgendwas nicht stimmt mit den Rechnungen, dann müssen wir auch die betreffenden Sachbearbeiter anrufen und fragen: Wie meintest du das jetzt? Oder: Hier fehlt was, oder die Fälligkeit kommt nicht mehr hin. Das war vorgestern, das geht so nicht, und solche Sachen. Wenn irgendwie Probleme auftreten, müssen wir dann direkt bei denen anrufen oder das manchmal sogar hinbringen, wenn's komplizierter wird.“
\end{abstract}

Sie mache das ganz gerne, berichtete die Befragte im Interview. So komme Abwechslung in ihre Arbeit und sie habe nicht nur mit Daten, sondern auch mit Menschen zu tun. Formal gedacht, könnte sie so manchen Beleg per Hauspost an die entsprechende Stelle zur Überarbeitung zurückschicken, wodurch unter Umständen Fristen versäumt oder Vorgesetzte auf fehlerhafte Arbeit aufmerksam würden.

„Wir sind sehr tolerant bei uns in der Sollerfassung. (...) Wenn es dringend ist, dann machen wir das auch sofort. Auch wenn das eigentlich nicht notwendig wäre, dass wir das in einer Stunde fertig haben müssen, sondern wir sagen immer so: drei Tage. Aber wir machen das dann schnell. Und ich würde sagen, da wir das dann immer alles so machen, so kulanterweise, dass wir dann auch der nette Posten sind.“

Die Befragte macht „kulanterweise“ mehr als sie muss. Sie arbeitet den Kolleginnen und Kollegen quasi entgegen, indem sie kleinere Fehler in der Arbeit anderer behebt und von sich aus nachfragt, um gemeinsam Probleme zu lösen. Sie erkennt selbst, wenn die Dinge eilig sind, und handelt im Sinne der Kolleginnen und Kollegen. In der Dankbarkeit der anderen sieht sie Bestätigung und Anerkennung.

Indirekt weisen die zitierten Interviewpassagen jedoch auch darauf hin, dass andere nicht immer so denken und handeln: „Wir sagen denen das zwar: Bitte füllt das richtig aus. Aber irgendwie wird das auch nicht richtig gemacht.“ Es könnte irgendwann der Punkt erreicht sein, wo die Befragte sich ausgenutzt fühlt, weil andere nachlässig oder - evtl. stressbedingt - fehlerhaft arbeiten, womit sie ihr zu viel zumuten und bei ihr für Überlastung sorgen. Das Gleichgewicht im Geben und Nehmen wäre gestört, vermutlich würde die Befragte „mit gleicher Münze heimzahlen“, indem sie „formal wird“ und beispielsweise Fehler auf den Belegen nur noch mit Rotstift markiert und das Ganze auf dem Dienstweg zurückschickt.

So weit ist es in einem Betrieb der Lebensmittelindustrie schon gekommen. Bei der Ablösung der Schichtgruppen gebe es ständig Konflikte - erklärten uns Arbeiterinnen und Arbeiter - darüber, wer zuständig ist für das Aufräumen am Arbeitsplatz. Wenn Stress herrscht, hinterlassen manche Kolleginnen und Kollegen ihren Arbeitsplatz unaufgeräumt, sodass die Beschäftigten der nachfolgenden Schicht erst einmal für Ordnung sorgen müssen, bevor sie mit ihrer eigentlichen Arbeit beginnen können. Normalerweise sei das kein Problem, weil jeder mal unter Stress geraten kann und auf das Entgegenkommen der anderen angewiesen ist.

Konflikte verursachten eher die Vorgesetzten, wurde uns gesagt. Offiziell gilt: Wer zu Schichtbeginn einen unaufgeräumten Arbeitsplatz oder eine ungesäuberte Maschine vorfindet, soll nicht die Arbeit übernehmen, sondern dem Vorgesetzten Meldung erstatten. Er wird dann die Person, die es bis zum Schichtende nicht geschafft hat, anweisen, noch dazubleiben, um Ordnung zu schaffen und sauberzumachen. Unmut darüber gibt es zum einen, 
weil diese Zeit nicht mehr als bezahlte Arbeitszeit gerechnet wird. Zum anderen, weil den Beschäftigten die kollektive Verantwortung für die Zusammenarbeit genommen wird und aus kollegialem Miteinander ein Gegeneinander gemacht wird.

\begin{abstract}
„Also Solidarität, die ist uns weggenommen worden, weil wir sind also - in Anführungsstrichen - schon dazu vergattert worden, bei der Schichtübergabe zu kontrollieren, ob alles in Ordnung ist. Wenn nicht alles in Ordnung ist, das sofort zu melden. Was hat das mit Solidarität zu tun? (...) Man wird gezwungen, den anderen schlecht zu machen, obwohl, mein Gott, wenn er das nicht geschafft hat, jetzt mit Sicherheit hat er einen Grund. Da kann ich mich auch für ihn einsetzen 'ne halbe Stunde.“
\end{abstract}

Im Verlauf eines Interviews schilderte uns ein Arbeiter den Konflikt etwas anders. Zwar meint auch er, normalerweise sei es kein Problem, mal etwas nachzuarbeiten, was die vorherige Gruppe nicht geschafft hat. Aber wenn das ständig vorkomme, fühle man sich ausgenutzt.

\begin{abstract}
„Eigentlich war es immer schon so, wenn wir eine bestimmte Gruppe abgelöst haben, dass wir also immer ein heilloses Chaos übernehmen. Und seit der letzten Woche haben wir einfach gesagt: So, Feierabend, wir gehen jetzt in die Offensive, das heißt, wenn die Schicht übernommen wird, hat das, das, das da zu stehen. Und wenn dem nicht so ist, dann bleibt derjenige, der die Schicht übergeben will, also sprich der Teigmacher, Anlagenführer oder so, bleibt der halt so lange da, bis alles im Reinen ist. Weil wir haben Produkte, die werden also in der Frühschicht gefahren, in der Frühschicht produziert, und dann kommt man manches Mal zur Nachtschicht, und (...) die Frühschicht hat's nicht geschafft, die Mittagsschicht hat's nicht geschafft, und Sie kommen dann zur Nachtschicht und müssen dann den Müll von der Frühschicht wegräumen.“
\end{abstract}

Wer auf diese Weise unkollegial handelt, muss mit einer „Offensive“ der anderen rechnen. Dann werden sie ihrerseits auch die Verantwortung ablehnen, sich auf Vorschriften berufen und den Vorgesetzten einschalten. Daraus lernen vielleicht die anderen, dass es ohne Rücksichtnahme nicht geht, dass alle Nachteile haben, wenn unkollegial gehandelt wird.

Gemeinsame Forderung der Beschäftigten, auf die sie sich mit dem Betriebsrat verständigt haben, ist, dass die eingeplanten und bezahlten Übergabezeiten verlängert werden, um Konflikte zu vermeiden. Bisher hat sich die Werksleitung vor allem aus Kostengründen noch nicht auf diese Forderung eingelassen.

Die Interviewpassagen zeigen, dass Zuständigkeiten für bestimmte Teilarbeiten zwar durch die offizielle Arbeitsorganisation vorgegeben und institutionalisiert werden können. Im konkreten Arbeitsprozess ergeben sich jedoch immer wieder Situationen, in denen es darauf ankommt, ob und wie Beschäftigte bei der eigenen Tätigkeit für andere mitdenken und so handeln, dass andere daran anschließen können. Als kollegial gilt, wenn die eigene Aufgabe nicht nur sorgfältig, sondern auch im Bewusstsein ausgeführt wird, dass sie Teil eines Zusammenhangs ist, der in Kooperation aktiv hergestellt werden muss und der ohne das eigene Zutun nicht herstellbar wäre. Es kommt darauf an, inwieweit gegenseitige Abhängigkeiten erkannt und in kollegiales Denken und Handeln umgesetzt werden. Kollegialität heißt in diesem Sinne, den anderen entgegenzuarbeiten und umgekehrt sich darauf zu verlassen, dass andere in gleicher Weise handeln.

Auch hier kommt es auf ein als ausgewogen empfundenes Geben und Nehmen an. Wer nur nach Vorschrift handelt, ohne sich um die Belange der anderen zu kümmern, wird Konflikte verursachen. Auch hier stoßen Beschäftigte unter Umständen auf strukturelle Hindernisse, wenn beispielsweise Vorschriften zu eng gefasst sind und keine Spielräume für kollegiales Handeln lassen. Probleme entstehen auch, wenn eine Seite das Entgegenkommen 
der anderen ausnutzt, um sich selbst die Mühe zu ersparen. Dann kann kollegiales Handeln in berechnendes, konkurrentes Verhalten umschlagen.

\subsection{Hilfe leisten}

Kollegiale Zusammenarbeit lebt davon, dass sich die Beteiligten gegenseitig helfen und unterstützen, auch wenn dies in keiner Regel und keiner Anordnung vorgeschrieben ist. Unter Kolleginnen und Kollegen hilft man sich, meinten Beschäftigte aus der Verwaltung eines Industriebetriebs. „So einfach. Hast du was falsch gemacht, versuchen wir, eine Lösung zu finden." Wer sieht, dass die bzw. der andere viel zu tun hat, fragt, ob er helfen kann. Als er einmal längere Zeit krank war, schilderte ein Befragter, habe er Schwierigkeiten gehabt, wieder in die Arbeit hineinzufinden. Die Kolleginnen und Kollegen haben ihm Zeit gelassen, haben ihm Arbeit abgenommen. „Da unterstützt man sich.“

Gegenseitige Hilfe wird problematisch, wenn keine Reserven vorhanden sind, wenn jeder Ausfall bei den anderen Stress verursacht. So kommt leicht eine unkollegiale Haltung auf. Ein Mitarbeiter aus einer Stadtverwaltung hat beobachtet, dass Fehler der Kolleginnen und Kollegen heute oft nicht mehr solidarisch behoben werden, sondern Anlass für gegenseitige Vorwürfe sind:

„Früher ist es ganz einfach so gewesen, wenn ein Fehler aufgetaucht ist, den hat man bereinigt, da hat man nicht drüber geredet. Und heute ist es also wirklich oft so, wenn man merkt, da ist was gelaufen, da kommt zuerst mal: ,Ich war's nicht, ich bin nicht zuständig. ““

Wird hier über einen Mangel an gegenseitiger Unterstützung geklagt, zeigt ein anderes Beispiel aus der gleichen Stadtverwaltung, dass Hilfe auch zu weit gehen kann. Eine Befragte berichtete davon, dass in ihrer Abwesenheit andere Kolleginnen und Kollegen ihre Telefonanrufe übernommen haben.

„Ich habe mir das verbeten, weil es führte nur dazu, dass jemand irgendwelche Auskünfte gab, die nur ich hätte geben können. Und sie waren am Ende verkehrt, und es hat nicht geholfen. Und deswegen habe ich gesagt: Wenn es bei mir klingelt, dann nimmt bitte keiner mehr ab.“

Hilfe kann Konflikte verursachen, wenn sie als Einmischung interpretiert wird, wenn Kompetenzen - im doppelten Sinn von Zuständigkeiten und Fähigkeiten - nicht beachtet werden. Zum einen bleibt die Verantwortung für eine mögliche Fehlleistung bei der zuständigen Person, was subjektiv als Hilfe gemeint ist, wird im objektiven Arbeitszusammenhang zur Zusatzbelastung. Zum anderen ist die Abgrenzung eines Zuständigkeitsbereichs ein Mittel, um den eigenen Status in der Organisation abzusichern, ein Eindringen wird als Bedrohung empfunden. Die Grenze zwischen Hilfe und Einmischung ist fließend, auch in dieser Hinsicht muss Kollegialität im täglichen Miteinander austariert werden.

Ein Arbeiter aus dem Lager eines Großhandelsbetriebs begründete seine positive Einschätzung der Zusammenarbeit in seiner Umgebung mit folgenden Worten:

„Ich denke mal, dieses miteinander sich austauschen, mal sprechen, auch in diesem Team agieren. Also wenn ich wirklich sehe, dass gerade jemand irgendwo sitzt oder der gerade nichts zu tun hat und dann wirklich so flott die Arbeit übernimmt, die dann gerade auf mich zukommt. Und ich weiß, die Arbeit wird mir gerade abgenommen, ich kann meiner Arbeit nachgehen wie auch immer, erleichtert das einen schon, macht den Kopf dementsprechend frei.“ 
Der Befragte lässt hier offen, warum er gerade überlastet ist und der Kollege weniger zu tun hat, sodass er übernehmen kann. Solche Diskrepanzen in der Auslastung können auf unterschiedliche Leistungsfähigkeit der Beschäftigten zurückzuführen sein. Leistungsunterschiede sind in gewissem Maße normal und werden im Arbeitsalltag kollegial ausgeglichen durch gegenseitige Unterstützung, können aber auch Anlass für Konflikte sein.

Konflikte über Leistung zeigten sich im Lager eines Industriebetriebes. Die Arbeit sei nicht selten mit Stress verbunden, erläuterte uns der Vertreter des Abteilungsleiters, weil es so viele verschiedene Packvorschriften gibt, die in der Regel von der Kundschaft vorgegeben werden. Wenn es schnell gehen muss, können einige Kolleginnen und Kollegen nicht mehr mithalten. Statt sich nun aber gegenseitig zu helfen, verhielten sich die Beschäftigten konkurrent zueinander:

„Es gibt da immer wieder Diskussionen: ,Ja, er macht erst das, der braucht da sechs Stunden für, ich schaffe
das in drei Stunden. Und da muss man sich natürlich solche Sachen auch anschauen, auch ins Gedächtnis ru-
fen und sagen: Moment mal, der ist sechzig, oder der ist fünfzig, und der ist zweiunddreißig, dreiunddreißig
oder fünfunddreißig. Das sind unter Umständen zwei verschiedene Welten. Und da die Kollegen eigentlich
recht lange schon bei uns im Lager sind, zehn Jahre oder so, dann kennt man die auch. Und man weiß auch:
Der hat das und das Wehwehchen und hier und da. Klar, immer muss man sagen: Nee, das geht nicht, man
muss trotzdem seine Leistung bringen, er kann sich da nicht drauf ausruhen. Aber wenn man dann mit ir-
gendjemand darüber diskutieren muss oder dazu gezwungen wird, dann muss man sich auch so was vor Au-
gen halten.“

Weil alle recht selbstständig arbeiten, hieß es in der Gruppendiskussion mit Beschäftigten aus dem Lager, entwickelten sich Unterschiede und Rivalitäten. Die Kolleginnen und Kollegen beobachteten sich gegenseitig, wer wie schnell und effektiv arbeitet, wer wie oft eine Pause macht usw. „Es gibt viele Kollegen, die achten mehr darauf, was die anderen machen, als auf die Arbeit.“

Eine Grundlage für solche Konkurrenzen ist die Tatsache, dass sich in dieser Abteilung ein bestimmter Leistungsbegriff durchgesetzt hat, der vom Unternehmen bzw. von den Vorgesetzten vorgegeben und von den Beschäftigten offenbar übernommen wurde. Als Leistung zählt die Menge der bewältigten Arbeit in einer gewissen Zeitspanne. Der Vorgesetzte berichtete uns im Interview, er strebe an, diese Unterschiede auch in einer Differenzierung des Lohns auszudrücken. Davon erhoffe er sich mehr Motivation für die Schwächeren und weniger Unzufriedenheit unter den Stärkeren.

Der Betriebsrat hat eine Lohndifferenzierung bislang verhindert, er vertritt die Auffassung, wer sich anstrengt und nach seinen Fähigkeiten arbeitet, solle dafür den vereinbarten Tariflohn erhalten. Unterschiede in der Leistungsfähigkeit sollten solidarisch von den Kolleginnen und Kollegen ausgeglichen werden. Jede und jeder brauche irgendwann mal die Hilfe der anderen.

Die Interviewpassagen zeigen, dass gegenseitige Hilfestellungen und Unterstützungsleistungen, so normal sie scheinen, keineswegs selbstverständlich sind. Voraussetzung ist zunächst eine gewisse Rücksichtnahme der Arbeitenden untereinander je nach persönlichen Eigenheiten, Stärken und Schwächen. Dazu gehört das Bewusstsein, selbst in einen Kooperationszusammenhang eingebunden zu sein und die eigene Arbeit ohne die zumindest zeitweilige Unterstützung von anderen nicht adäquat ausführen zu können.

Es wird aber auch deutlich, wie Leistungsdenken die Entwicklung von Kollegialität behindern kann, wenn ergebnisorientierte Leistungsmaßstäbe angelegt werden, die auf in- 
dividuelle Unterschiede abheben. An die Stelle gegenseitiger Unterstützung tritt ein Aufrechnen der jeweiligen Beiträge. Hier liegt ein wesentliches strukturelles Hindernis für die Entwicklung von Kollegialität, das nur durch allgemeine Regelungen beispielsweise in einer Betriebsvereinbarung zu beseitigen wäre. Hindernisse zeigen sich auch dort, wo aufgrund von Arbeitsüberlastungen die Ressourcen für gegenseitige Hilfeleistungen fehlen. Wer selbst in Hektik ist, wird sich nicht die Zeit nehmen, der Kollegin oder dem Kollegen zur Seite zu springen. Hilfestellung erfordert eine Personaleinsatzplanung, die Handlungsspielräume für die Beschäftigten lässt.

\section{Voraussetzungen und Folgen kollegialen Handelns - ein Fazit}

Im vorliegenden Beitrag wurde gezeigt, dass sich parallel und im Widerspruch zu konkurrenzbedingten Fragmentierungen im kooperativen Arbeitsprozess immer auch Gelegenheiten für wechselseitige zwischenmenschliche Bindungen und Verpflichtungen ergeben. Es wurden Praktiken analysiert, mit denen Arbeitende ihre sozialen Beziehungen interaktiv in reziproken Austauschprozessen gestalten und zu Kollegialität im Sinne eines FüreinanderEinstehens formen können.

- Grundlage kollegialer Beziehungen ist das Bemühen, die Arbeitsaufgaben als gemeinsame zu definieren und Einvernehmen darüber zu erzielen, was als Ziel der Zusammenarbeit gelten soll.

- Zusammenarbeit als soziale Beziehung erfordert ein gewisses Verständnis der Personen füreinander, das spontan entstehen kann, aber in der Regel bewusst von den Beteiligten hergestellt und geformt wird.

- $\quad$ Eine dritte Praktik zur Herstellung kollegialer Beziehungen besteht darin, dass die Arbeitenden an den Schnittstellen ihrer jeweiligen Tätigkeiten einander entgegenkommen.

- Schließlich entwickeln die Beschäftigten Praktiken gegenseitiger Hilfe und Unterstützung.

Diese Prozesse sind voraussetzungsvoll und werden ständig sowohl von strukturellen Gegebenheiten wie auch von gegenläufigen Prozessen der Abgrenzung und gegenseitigen Instrumentalisierung durchkreuzt. Kollegialität beruht auf Erfahrung und Einsicht, dass die Realisierung eigener Ziele von der Realisierung der Ziele anderer abhängig ist, dass die eigene Handlungsfähigkeit in der Zusammenarbeit und im reziproken Austausch mit anderen erweitert werden kann. Kollegialität als eine Form von Solidarität entsteht weder aus einer gegebenen Gemeinschaft noch aus funktionalen Erfordernissen des Arbeitsprozesses. Kollegialität wird vielmehr in Interaktionen generiert und - wenn sie Bestand haben soll - immer wieder gegen Tendenzen der Abgrenzung und gegenseitigen Instrumentalisierung durchgesetzt.

Es konnten einige Bedingungen herausgearbeitet werden, die Praktiken kollegialen Handelns entweder begünstigen oder behindern. In einigen Interviewpassagen wurde deutlich, dass bei der Gestaltung entsprechender Strukturen die Betriebsräte eine wesentliche Rolle spielen. 
- Kollegialität beruht auf einer bewussten Gestaltung sozialer Beziehungen entlang gegebener Zusammenhänge und Abhängigkeiten der Arbeitsvorgänge. Strukturelle Voraussetzung ist deshalb zunächst eine gewisse Transparenz betrieblicher Abläufe und Arbeitsteilungen insbesondere außerhalb der jeweils eigenen Gruppe. Dort, wo nicht nachvollzogen werden kann, „was die da eigentlich machen“, entwickelt sich eher abgrenzendes und konkurrentes Handeln. Ein Betriebsrat, der sich in der Regel aus Beschäftigten unterschiedlicher Abteilungen zusammensetzt, kann hier informierend und vermittelnd eingreifen.

- Eine weitere Bedingung für die Entwicklung von Kollegialität ist die Verfügung über genügend Zeitressourcen, um „über den Tellerrand“ der eigenen Tätigkeit hinaus zu sehen und Interesse für die Arbeit der Kolleginnen und Kollegen zu zeigen. Wenn die Personal-, Arbeits- und Zeitplanung nach Effizienzgesichtspunkten nur die formale, berechenbare Seite des funktional Notwendigen berücksichtigt, können sich die spontanen, nicht formalisierbaren Praktiken zur Entwicklung sozialer Beziehungen nur schwer entfalten. In dieser Hinsicht kann eine Einflussnahme des Betriebsrats auf Personalpolitik und Arbeitsgestaltung positiv auf die Entwicklung von Kollegialität hinwirken.

- Soziale Beziehungen beruhen auf Kommunikation der Individuen in der Arbeitsgruppe und darüber hinaus. Einvernehmen über Ziele und Formen der Zusammenarbeit wird vorwiegend diskursiv erzeugt, auch gegenseitiges Verständnis erfordert sprachliche Kommunikation über das funktional Notwendige hinaus. Kommunikation ist Bestandteil der Arbeit. Wo diese Einsicht fehlt und spontane Kommunikation strukturell oder durch Vorgesetzte behindert wird, können sich kollegiale Beziehungen nur schwer entwickeln, was in der Regel auch zu sachlichen Problemen in der Zusammenarbeit führt. Betriebsratsmitglieder können einerseits selbst durch Gespräche vor Ort für Kommunikation sorgen, andererseits durch Regelung von Arbeitszeit und Leistungsbedingungen die zur Kommunikation notwendigen „Poren“ im Arbeitsalltag schaffen.

- Das Leistungsprinzip ist ein Einfallstor für konkurrentes Handeln. Wo Leistung am Ergebnis gemessen wird, kann jemand, der bzw. die weniger leistungsfähig ist, als Hindernis der Zusammenarbeit erscheinen. Kollegialität entwickelt sich eher dort, wo Unterschiede akzeptiert und durch gegenseitige Unterstützung ausgeglichen werden. Die Mitbestimmung des Betriebsrats bei der Leistungsbeurteilung kann die Zusammenarbeit fördern und einzelne Beschäftigte vor Ausgrenzung schützen.

- Kollegiales Handeln in der Gruppe kann zugleich eingesetzt werden als Mittel der Abgrenzung gegenüber anderen Gruppen oder Individuen. Letzteres wird begünstigt in Formen der betrieblichen Sozialordnung, die durch Machtkämpfe und mikropolitisches Taktieren gekennzeichnet sind. Kollegialität wird eher begünstigt in Verhältnissen mit verlässlichen, ausgehandelten Regelungen. Genau hierin - durch Betriebsvereinbarungen Willkür zu begrenzen und für mehr Gerechtigkeit zu sorgen - liegt eine der wichtigsten Aufgaben eines Betriebsrats.

Inwieweit könnten betrieblich gewonnene Erfahrungen und Einsichten kollegialen Handelns verallgemeinert werden auf die Gestaltung solidarischer Beziehungen in Gewerkschaft und Gesellschaft? Hierzu sollen abschließend noch einige Überlegungen angestellt werden, die über das hier ausgewertete empirische Material hinausgehen. 
Analysen zur aktuellen Gewerkschaftspolitik zeigen, dass die Organisationen nicht mehr nur an Solidarität appellieren, sondern sich stärker darauf orientieren, Solidarität in offenen Diskussionsprozessen und aktiven Auseinandersetzungen hervorzubringen. Arbeitskämpfe werden stärker so angelegt, dass Gewerkschaftsmitglieder eigenständig solidarisch handeln, statt nur dem Aufruf des Vorstands Folge zu leisten. Betrieblich aktive Gewerkschaftsmitglieder und Betriebsräte sind bestrebt, ihr eigenes Handeln durch Rückkopplung mit den Belegschaften zu legitimieren und den Kreis der gewerkschaftlich Aktiven zu vergrößern (Dörre et al., 2016, S. 217-249; Bahnmüller \& Salm, 2018). Auf Seiten der Belegschaften scheint die Bereitschaft, über den Betrieb hinaus gemeinsam mit anderen für die eigenen Forderungen einzutreten, dort größer zu sein, wo schon im betrieblichen Alltag Kollegialität praktiziert wird (Dörre et al., 2016, S. 156-157 und S. 167-168). Basis dafür ist stärker als früher die gemeinsame Erfahrung von Ausbeutung, Ungerechtigkeit und eines damit verbundenen Zwangs zur Konkurrenz. „Die Streiks von 2015 belegen, dass dieses wohl lange latente Ungerechtigkeitsbewusstsein zur subjektiven Triebkraft von gewerkschaftlicher Organisierung und kollektiver Handlungsfähigkeit werden kann“ (ebd., S. 186).

Allerdings lässt sich - wie auf der betrieblichen Ebene - auch hier feststellen, dass Solidarität exklusiv gestaltet werden kann (Dörre et al., 2011; Dörre \& Matuschek, 2013). Insbesondere in Krisensituationen gehen Beschäftigte und Betriebsräte „Wettbewerbskoalitionen“ mit dem Management ein, um gemeinsam auf den Märkten zu bestehen und das Erreichte im Betrieb abzusichern. Die Gewerkschaft wird von vielen zwar als Interessenvertretung akzeptiert, was jedoch nicht zwingend mit einer Bereitschaft zu inklusiver gruppen- und betriebsübergreifender Solidarität einhergeht. Eine große Gruppe der von Dörre et al. befragten Beschäftigten äußert die Ansicht, es habe keinen Sinn mehr, mit möglichst vielen gemeinsam für Verbesserungen einzutreten, es gehe vielmehr darum, für sich selbst das Erreichte zu sichern und gegen andere zu verteidigen. „Eine gemeinsame Aufstiegsperspektive, die auch den Outsidergruppen Chancen eröffnet, wird nicht gesehen. Stattdessen gilt es nach Auffassung der Befragten, den Status Quo zu bewahren. Aus einer solchen Perspektive heraus wird die IG Metall subjektiv zum Instrument selektiver Interessenpolitik. Sie dient den Festangestellten dazu, ihren sozialen Status zu verteidigen. Positiver Gewerkschaftsbezug und exkludierende Solidarität gehen eine überraschende Wahlverwandtschaft ein“ (Dörre et al., 2011, S. 41).

Einen der Hauptgründe für solche Tendenzen exklusiver Solidarität sehen Dörre und Matuschek (2013, S. 50-52) darin, dass die von vielen als ungerecht angesehene Gesellschaft zugleich als unveränderbar, als Ausdruck von Sachzwängen interpretiert wird. Interessenpolitik erscheint als Nullsummenspiel, wer gewinnen will, muss anderen etwas nehmen. Den befragten gewerkschaftlich aktiven Gruppen gelingt es nur unzureichend, eine Verbindung vom betrieblichen, kollegial mitgestalteten Mikrokosmos zum scheinbar fest gefügten gesellschaftlichen Makrokosmos herzustellen.

Diesen Erkenntnissprung zu unterstützen und - basierend auf den Erfahrungen der Gewerkschaftsbewegung - zu zeigen, dass gesellschaftliche Verhältnisse durch solidarischen Zusammenschluss und gemeinsames Handeln verändert werden können, ist weiterhin eine der zentralen Aufgaben kritischer Sozialwissenschaft. Das soziologische Denken, schreibt Zygmunt Bauman (2000, S. 29) ,verfügt über eine eigene Macht: die Macht, Verfestigtes aufzulösen. Es bringt die scheinbar fixierte und erstarrte Welt wieder in Bewegung; es weist darauf hin, dass die Welt anders sein könnte, als sie ist.“ 


\section{Literatur}

Bachmann, G. (2014). Kollegialität. Eine Ethnographie der Belegschaftskultur im Kaufhaus. Frankfurt a.M.: Campus.

Bahnmüller, R. \& Salm, R. (2018). Beteiligung und Tarifpolitik: Debatten, Ansätze und Grenzen am Beispiel der IG Metall. Industrielle Beziehungen, 25 (1), 27-50. doi: 10.3224/indbez.v25i1.02

Bauman, Z. (2000). Vom Nutzen der Soziologie. Frankfurt a.M.: Suhrkamp.

Bayertz, K. (Hrsg.). (1998). Solidarität - Begriff und Problem. Frankfurt a.M.: Suhrkamp.

Bierhoff, H. \& Küpper, B. (1999). Das „Wie” und „Warum” von Solidarität. Bedingungen und Ursachen der Bereitschaft zum Engagement für andere. Ethik und Sozialwissenschaften, 10 (2), 181 255.

Billmann, L. \& Held, J. (Hrsg.). (2013). Solidarität in der Krise. Gesellschaftliche, soziale und individuelle Voraussetzungen solidarischer Praxis. Wiesbaden: VS-Verlag. doi: 10.1007/978-3-658-00912-0

Böhle, F. \& Bolte, A. (2002). Die Entdeckung des Informellen. Der schwierige Umgang mit Kooperation im Arbeitsalltag. Frankfurt a.M.: Campus.

Bolte, A., Neumer, J. \& Porschen, S. (2008). Die alltägliche Last der Kooperation. Abstimmung als Arbeit und das Ende der Meeting-Euphorie. Berlin: edition sigma. doi: 10.5771/9783845268729

Bolte, A. \& Porschen, S. (2006). Die Organisation des Informellen. Modelle zur Organisierung von Kooperation im Arbeitsalltag. Wiesbaden: VS-Verlag.

Caillé, A. (2008). Anthropologie der Gabe. Frankfurt a.M.: Campus.

Daser, B. (2011). Kollegialität. In R. Haubl \& G. Voß (Hrsg.), Riskante Arbeitswelten im Spiegel der Supervision (S. 38-45). Göttingen: Vandenhoeck \& Ruprecht.

Dörre, K., Hänel, A., Holst, H. \& Matuschek, I. (2011). Guter Betrieb, schlechte Gesellschaft? Arbeits- und Gesellschaftsbewusstsein im Prozess kapitalistischer Landnahme. In C. Koppetsch (Hrsg.), Nachrichten aus den Innenwelten des Kapitalismus (S. 21-49). Wiesbaden: VS-Verlag. doi: 10.1007/978-3-531-93482-2_2

Dörre, K. \& Matuschek, I. (2013). Kapitalistische Landnahmen, ihre Subjekte und das Gesellschaftsbild der LohnarbeiterInnen. In K. Dörre, A. Happ \& I. Matuschek (Hrsg.), Das Gesellschaftsbild der LohnarbeiterInnen. Soziologische Untersuchungen in ost- und westdeutschen Industriebetrieben (S. 29-53). Hamburg: VSA.

Dörre, K., Goes, T., Schmalz, S. \& Thiel, M. (2016). Streikrepublik Deutschland? Die Erneuerung der Gewerkschaften in Ost und West. Frankfurt a. M.: Campus.

Hénaff, M. (2009). Der Preis der Wahrheit. Frankfurt a. M.: Campus.

Hillebrandt, F. (2009). Praktiken des Tauschens. Zur Soziologie symbolischer Formen der Reziprozität. Wiesbaden: VS-Verlag. doi: 10.1007/978-3-531-91693-4

Holzkamp, K. (1983). Grundlegung der Psychologie. Frankfurt a.M.: Campus.

Hondrich, K. \& Koch-Arzberger, C. (1992). Solidarität in der modernen Gesellschaft. Frankfurt a.M.: Fischer.

Honneth, A. (2008). Arbeit und Anerkennung - Versuch einer Neubestimmung. Deutsche Zeitschrift für Philosophie, 56 (3), 327-341.

Hürtgen, S. (2013). Mensch sein auf der Arbeit? Kollegialität als Balance von allgemein-menschlichen und leistungsbezogenen Aspekten von Arbeit. In L. Billmann \& J. Held, J. (Hrsg.), Solidarität in der Krise. Gesellschaftliche, soziale und individuelle Voraussetzungen solidarischer Praxis (S. 237-262). Wiesbaden: VS-Verlag. doi: 10.1007/978-3-658-00912-0_11

Jaeggi, R. (2017a). Pathologies of Work. Woman's Studies Quarterly, 45 (3\&4), 59-76. doi: $10.1353 /$ wsq.2017.0044

Jaeggi, R. (2017b). Solidarität und Gleichgültigkeit. In Artists Unlimited, A. Jaehle \& P. Buckermann (Hrsg.), Kinship in Solitude. Perspectives on Notions of Solidarity (S. 87-102). Hamburg: adocs. 
Jaeggi, R. \& Celikates, R. (2017). Sozialphilosophie. Eine Einführung. München: C.H.Beck.

Jaeggi, R. \& Kübler, L. (2014). Pathologien der Arbeit. Zur Bedeutung eines gesellschaftlichen Kooperationsverhältnisses. WSI-Mitteilungen, 67 (7), 521-527. doi: 10.5771/0342-300X-2014-7-521

Kock, K. \& Kutzner, E. (2014). „Das ist ein Geben und Nehmen“ - Eine empirische Untersuchung über Betriebsklima, Reziprozität und gute Arbeit. Berlin: edition sigma.

Komter, A. (2005). Social Solidarity and the Gift. Cambridge: Cambridge University Press.

Krömmelbein, S. (2004). Kommunikativer Stress in der Arbeitswelt. Zusammenhänge von Arbeit, Interaktion und Identität. Berlin: editon sigma.

Laitinen, A. \& Pessi, A. (Hrsg.) (2014). Solidarity. Theory and Practice. Lanham etc.: Lexington Books.

Lohr, K. (2017). Subjektivierung von Arbeit. In H. Hirsch-Kreinsen \& H. Minssen (Hrsg.), Lexikon der Arbeits- und Industriesoziologie (2. Auflage, S. 281-285). Berlin: Nomos. doi: $10.5771 / 9783845276021-281$

Markard, M. (2005). Einführung in die kritische Psychologie. Berlin: Argument Verlag.

Petersdorff, W. v. (2017). Schluss mit Homeoffice. IBM holt die Mitarbeiter zurück ins Büro. Abgerufen von: faz-net.de (Artikel vom 19.05.2017), abgerufen am 3.4.2018

Projektgruppe Automation und Qualifikation (1986). Widersprüche der Automationsarbeit. Ein Handbuch. Berlin: Argument Verlag.

Pulcini, E. (2004). Das Individuum ohne Leidenschaften. Berlin: Diaphanes.

Smith, N. (2014). Solidarity and Work. A Reassessment. In A. Laitinen \& A. Pessi (Hrsg.), Solidarity. Theory and Practice (S. 155-177). Lanham etc.: Lexington Books.

Spieß, E. (2007). Kooperation und Konflikt. In H. Schuler \& K. Sonntag (Hrsg.), Handwörterbuch der Arbeits- und Organisationspsychologie (S. 339-347). Göttingen etc.: Hogrefe.

Süß, R. (2015). Kollektive Handlungsfähigkeit. Gramsci - Holzkamp - Laclau, Mouffe. Wien, Berlin: Turia + Kant.

Zoll, R. (2000). Was ist Solidarität heute? Frankfurt a.M.: Suhrkamp. 


\section{Kampfmethode Ultimatum. Von disziplinierender Kollegialität zu widerständiger Solidarität - Fallbeispiele aus dem Gesundheitswesen**}

\section{Zusammenfassung}

Was ist Solidarität im Krankenhaus? Im Alltag äußert sie sich darin, dass Pflegekräfte die eigenen Schutzrechte ignorieren, um Patientinnen und Patienten, aber auch ihre Kolleginnen und Kollegen „,nicht hängen zu lassen“. Der Beitrag handelt von dem Versuch, aus dieser disziplinierenden Form der Kollegialität eine „Kampf-Solidarität“ zu entwickeln: Teams fordern ultimativ Maßnahmen zur Entlastung und drohen damit, andernfalls freiwillige Leistungen wie das Einspringen außerhalb des Dienstplans zu verweigern. Sie nutzen ihre gestiegene Produktionsmacht, da die Abläufe im Krankenhaus regelhaft darauf basieren, dass sich Pflegekräfte über ihre arbeitsvertraglichen Pflichten hinaus engagieren. Der Beitrag untersucht diese neue Kampfform der Ultimaten anhand von sechs Fallstudien, basierend unter anderem auf Experteninterviews mit Pflegekräften, Gewerkschaftssekretär/innen und betrieblichen Interessenvertreter/innen. Theoretisch knüpft er an Solidaritätskonzeptionen von Bayertz (1998) und den Machtressourcenansatz an. Er kommt zu dem Schluss, dass die Ultimaten dazu führen können, die - wie es ein Krankenpfleger und ver.di-Aktivist ausdrückt - im Klinikalltag ,,auf den Kopf gestellte“ Solidarität „,wieder auf die Füße“ (SWB04, 30-32) ${ }^{1}$ zu stellen. Es entwickelt sich eine Kampf-Solidarität, die die Durchsetzungsfähigkeit in Arbeitskämpfen, aber auch die Bereitschaft zu widerständigem Alltagsverhalten stärkt.

Schlagwörter: Solidarität, Machtressourcen, Gesundheitswesen, Arbeitskampf

* Dr. Daniel Behruzi, Wissenschaftlicher Mitarbeiter an der Technischen Universität Darmstadt, Institut für Soziologie, Dolivostr.15, 64293 Darmstadt. E-Mail: behruzi@ifs.tu-darmstadt.de

** Artikel eingegangen: 15.05.2018, revidierte Fassung akzeptiert nach doppelt-blindem Begutachtungsverfahren: 12.09 .2018

1 Die Interview-Codes beginnen mit der Bezeichnung der Region ( $\mathrm{S}=$ Saarland, SW=Baden-Württemberg; $\mathrm{C}=$ Berlin); in der Mitte steht $\mathrm{B}$ für Beschäftigter und V für Gewerkschaftssekretär/in; am Ende sind die Codes durchnummeriert. Die Zahlen nach dem Komma bezeichnen den Absatz des transkribierten Interviews in MaxQDA. 


\title{
The Ultimatum method of industrial action. From disciplinary collegiality to resistant solidarity-case studies in the health care sector
}

\begin{abstract}
What is solidarity in hospitals? In everyday life, it expresses itself in the fact that nursing staff ignore their own protective rights in order not to "let down" patients as well as colleagues. This contribution examines the attempt to develop "employee solidarity" in the face of the disciplinary nature of collegiality: Teams ultimately demand measures for relief or otherwise threaten to refuse to work hours not laid down in the staff roster. Here, the workforces' bargaining power is enhanced by the fact that hospitals regularly depend upon the engagement of nursing staff beyond their contractual obligations. To this end, the article applies Bayertz' (1998) concept of solidarity and the power resources approach. It comes to the conclusion that ultimatums can have the effect-as posited by a nurse and trade union officer-to put solidarity, which is "turned upside down" in everyday life in hospitals, "back on its feet" (SWB04, 30-32). Such a form of solidarity has the advantage that it strengthens the assertiveness of industrial action as well as increases employees' willingness to resist in their everyday working life. The contribution examines this new ultimatum method of industrial action by drawing on research findings taken from six case studies. This involved interviews with nursing staff, trade union secretaries and works council members.
\end{abstract}

Keywords: Solidarity, power resources, health sector, industrial disputes (JEL: I5, J5)

\section{Einleitung $^{2}$}

„Unbefristete Streiks an Uniklinika Essen und Düsseldorf“ (Bibliomed Pflege vom 6. August 2018); „Ver.di droht mit langem Streik an Uniklinik Homburg“ (Kirch, 2018). Das sind zwei aktuelle Schlagzeilen, die noch vor einigen Jahren für unmöglich gehalten worden wären. Sie dokumentieren: Die Industriellen Beziehungen im Gesundheitswesen sind in Bewegung geraten. Bis nach der Jahrhundertwende waren Arbeitskämpfe in dieser Branche de facto nicht existent. Sie beschränkten sich auf eine zumeist symbolische Beteiligung von Krankenhausbeschäftigten an den Tarifrunden des öffentlichen Dienstes, dessen Tarifwerk die Arbeitsbedingungen weit über die kommunalen Krankenhäuser und landeseigenen Unikliniken hinaus prägte. Ein Grund für die weitgehende Absenz von Arbeitskonflikten im Gesundheitswesen lag in dessen Organisations- und Eigentumsverhältnissen: Anders als in der Privatindustrie fällt ein Großteil der Krankenhäuser unter staatliche oder freigemeinnützige Trägerschaften, die keine Profitabsichten verfolgen. In konfessionellen Häusern gelten Tarifverhandlungen und Streikrecht bis heute als mit dem kirchlichen Selbstordnungsrecht unvereinbar (Bingener, 2012). Diese Grundkonstellation besteht trotz der Expansion privater Träger und marktwirtschaftlich getriebener Veränderungen in der Krankenhausfinanzierung (siehe Abschnitt 2.3) fort. Dennoch ist eine zunehmende Konvergenz der Konfliktkonstellationen im Krankenhausbereich mit denen in der Privatwirtschaft festzustellen. Wie ist das zu erklären? Was hat sich geändert? 
Dieser Beitrag ist Teil einer Suchbewegung, die sich den Ursachen für diese Entwicklung annähert. Er hat nicht den Anspruch, eine umfassende Analyse der Verhältnisse und Arbeitsbeziehungen im Gesundheitswesen zu liefern. Viele relevante Aspekte können nicht systematisch aufgearbeitet werden. Das Forschungsinteresse liegt in den neuen Kampfkonstellationen und -methoden, die sich in jüngster Zeit entwickelt haben. Dass Krankenhausbetten und ganze Stationen durch Streiks geschlossen werden müssen - zuletzt beispielsweise an der Uniklinik Essen (Simon, 2018) - ist ein Novum, dessen Hintergründe es zu untersuchen gilt. Der spezielle Fokus dieses Beitrags liegt allerdings auf einer neuartigen Kampfform, die unterhalb der Schwelle formaler Arbeitskämpfe angesiedelt ist - dem sogenannten Ultimatum. Mit dieser Methode wehren sich einzelne Teams gegen Überlastung - zumeist aus der Krankenhauspflege, in Einzelfällen aber auch aus anderen Beschäftigtengruppen. Sie drohen damit, ab einem bestimmten Zeitpunkt Leistungen zu unterlassen, zu denen sie per Arbeitsvertrag nicht verpflichtet sind. So lehnen sie es beispielsweise ab, auBerhalb des Dienstplans einzuspringen, während unbezahlter Pausen zu arbeiten, Überstunden zu machen, zusätzliche Verantwortlichkeiten oder ärztliche Tätigkeiten zu übernehmen. Dies drohen sie für den Fall an, dass die Geschäftsleitung bis zum genannten Datum konkrete Forderungen nicht erfüllt, die zu einer Entlastung führen sollen. Da die Abläufe in den Krankenhäusern aufgrund der dünnen Personaldecke auf solchen freiwilligen Leistungen der Pflegekräfte basieren, übt die Verweigerungsdrohung einen gehörigen Druck auf die Klinikleitung aus. Die Pflegeteams sind so in vielen Fällen in der Lage, Zugeständnisse und Verbesserungen durchzusetzen.

In der Literatur wird vielfach beschrieben, wie der „Pflegeethos“ (Senghaas-Knobloch, 2008) Pflegekräfte dazu bringt, ihre Gesundheit aufs Spiel zu setzen, weil sie ihre Patient/innen nicht im Stich lassen wollen. Der Fokus des vorliegenden Beitrags liegt jedoch auf einem anderen Faktor: der alltäglichen Kollegialität, der „Solidarität“ mit den anderen Mitgliedern des Teams. Sie spielt unseren Erkenntnissen zufolge eine mindestens ebenso wichtige Rolle dabei, dass Beschäftigte ihre Rechte nicht wahrnehmen und „freiwillig“ gesetzliche, einzel- und tarifvertragliche Grenzen überschreiten. Die zentrale These ist, dass diese disziplinierende Kollegialität im Zuge der neuen Kampfmethoden in eine widerständige „Kampf-Solidarität“ transformiert werden kann.

Empirische Basis sind sechs Fallstudien: Untersucht werden jeweils zwei Stationen aus Krankenhäusern im Saarland, im Raum Stuttgart und in Berlin. Diese Regionen wurden auf Grundlage von Vorgesprächen und vorhandenem Feldwissen als diejenigen identifiziert, in denen die Ultimatum-Methode am häufigsten angewandt wird. Die Fallbeispiele wurden mit Blick auf größtmögliche Vergleichbarkeit ausgewählt: Es handelt sich bei allen um große öffentliche Krankenhäuser, in denen ver.di vergleichsweise gut organisiert ist. Andere Fälle, zum Beispiel aus der Altenpflege, wurden aus Gründen der Vergleichbarkeit nicht berücksichtigt.

Die Untersuchung basiert auf einem Dutzend informeller Gespräche sowie 13 leitfadengestützten Interviews mit Expertinnen und Experten, aber auch auf der Auswertung von Flugblättern, Betriebszeitungen, Vereinbarungen, Broschüren und Dokumentationen. Mittels informeller Vorgespräche wurden das Terrain sondiert, die Fallbeispiele ausgewählt und ein Leitfaden entwickelt. In jeder Untersuchungsregion wurden mit jeweils den zuständigen ver.di-Sekretären und an den Ultimaten beteiligten Pflegekräften sowie Betriebs- und 
Personalräten problemzentrierte Experteninterviews (Mayring, 2002, S. 67) geführt. Ebenso mit einer Funktionärin der ver.di-Bundesverwaltung, um eine überregionale Betrachtung der Geschehnisse zu ermöglichen. Die Experteninterviews beschreiben und bewerten Sachverhalte also aus unterschiedlichen Perspektiven. Sie wurden mit Hilfe des Softwareprogramms MaxQDA einer qualitativen Inhaltsanalyse unterzogen, die sich an Mayring (1994; 2002, S. 114-121) sowie den Einführungen von Cropley (2002, S. 127-136) und Lamnek (2005, S. 517-531) orientiert.

Ein Kritikpunkt am Fallstudien-Ansatz ist das mögliche Fehlen externer Validität (Cropley, 2002, S. 96). Um diese zu gewährleisten, sollten die Untersuchungsobjekte so gewählt sein, dass sie ,hinsichtlich einer gleich oder ähnlich strukturierten größeren Menge von Phänomenen als typische Fälle oder besonders prägnante oder aussagefähige Beispiele gelten“ (Hartfiel \& Hillmann, 1972, S. 160). Letzteres gilt auch für die hier versammelten Fallstudien. Sie zielen nicht auf Repräsentativität, sondern sind Teil einer Suchstrategie, die Hinweise auf die Erklärung neuartiger Phänomene liefern soll.

In den Abschnitten 2.1 und 2.2 werden zunächst theoretische Vorüberlegungen zu Solidaritätskonzeptionen und zum Machtressourcenansatz angestellt, die als analytische Folien dienen. In Abschnitt 2.3 werden Prozesse der Ökonomisierung und der Indirekten Steuerung dargestellt, die als Grundlage für die Entstehung neuer Kampfformen gelten können. In Abschnitt 3 folgen die Fallerzählungen. Die Erkenntnisse zu Prozessen und Akteuren werden in Abschnitt 4 verdichtet und systematisch zusammengefasst. In Abschnitt 5 erfolgt eine Diskussion der Ergebnisse, bevor in Abschnitt 6 ein Fazit gezogen wird.

\section{Theoretische Vorüberlegungen}

\subsection{Solidarität}

Schon seit Durkheim (1992 [1893]) spielt das Thema Solidarität in der Soziologie eine zentrale Rolle. Dennoch existiert kein fest umrissenes analytisches Konzept. Vielmehr „,wird in einzelnen Analysen auf sehr unterschiedliche und zum Teil widersprüchliche Solidaritätsbegriffe zurückgegriffen“, wie Tranow (2012, S. 13) in seinem Versuch einer handlungstheoretischen Fundierung des Begriffs betont. Es gilt daher zunächst, den diesem Beitrag zugrundeliegenden Solidaritätsbegriff darzustellen, ohne dabei den Anspruch einer umfassenden Diskussion der Thematik erheben zu wollen.

Bayertz (1998) unterscheidet (neben weiteren) zwischen zwei Typen von Solidarität: „Gemeinschafts-Solidarität“ bezieht sich auf wechselseitige Bindungen und Verpflichtungen innerhalb partikularer Gemeinschaften. „Kampf-Solidarität“ hingegen definiert er als „die Bereitschaft eines Individuums oder einer Gruppe [...], einem anderen Individuum oder einer anderen Gruppe bei der Durchsetzung seiner oder ihrer Rechte zu helfen“ (Bayertz, 1998, S. 49). Sie ist nicht nur, wie auch andere Formen der Solidarität, exklusiv, insofern Individuen oder Gruppen mit anderen Zielen und Interessen ausgeschlossen werden. Sie ist auch konflikthaft, da das gemeinsame Handeln in der Regel erst dadurch notwendig wird, dass ,zur Realisierung der gemeinsamen Ziele und Interessen Widerstände überwunden werden müssen; und zwar vor allem Widerstände anderer Gruppen, die konkurrierende Interessen vertreten“ (Bayertz, 1998, S. 41). 
Historisch erwächst Kampf-Solidarität auf Basis gemeinsamer (Klassen-) Interessen, „,im Wissen um die gleichartige Betroffenheit durch die soziale Konfiguration des Industriekapitalismus“ (Tenfeld, 1998, S. 197). Sie unterscheidet sich von Wohltätigkeit dadurch, dass „eine grundsätzliche Gleichheit zwischen den Beteiligten existiert, die zu der gegenseitigen Erwartung von Hilfe im Bedarfsfall berechtigt“ (Bayertz, 1998, S. 41, Hervorhebung im Original), sie ist also latent reziprok, basiert aber ,auf Freiwilligkeit, nicht auf Zwang“ (Scherr, 2013, S. 265). Ein bloßes gemeinsames „materielles Interesse“ ist indes nicht hinreichend. Anders als im rational choice Ansatz (Hechter, 1988) besitzt Solidarität für Bayertz auch eine „uneliminierbare moralische Dimension“, da sie der Durchsetzung legitimer, „gerechter Ziele“ dient (Bayertz, 1998, S. 44-45, Hervorhebung im Original). Auch Wildt (1998, S. 211) erklärt mit Bezug auf Honneth (1992) und Van Parijs (1995, 1996), „dass zur Solidarität erstens eine besondere Form von moralischer Motivation oder Altruismus gehört und zweitens ein Bezug auf Kooperation oder jedenfalls auf Wechselseitigkeit".

Mit Tranow (2012, S. 36) gehe ich davon aus, dass soziologisch interessierende Solidarität stets einen Handlungsbezug aufweist und nicht allein Gefühlsregungen beschreibt. Laut Billmann \& Held (2013, S. 24) ist Solidarität allerdings ,nicht nur eine Handlung, sondern auch eine Haltung, und manchmal auch nur ein Gefühl der Verbundenheit“. Sie beruht nicht nur auf Interessen, sondern auch auf Identitäten (Baum, 1975).

„Kampf-Solidarität“ ist von einer im betrieblichen Alltag gelebten Kollegialität zu unterscheiden. Analog zu Thomes (1998) Differenzierung zwischen funktionaler und normativer Integration basiert Alltagskollegialität auf der Notwendigkeit von Kooperation im Arbeitsprozess, also auf „Verschiedenheit“, während „Kampf-Solidarität““ auf „Gemeinsamkeiten" fußt - in diesem Fall dem kollektiven Ziel besserer Arbeitsbedingungen. Hürtgen (2013) differenziert in Bezug auf Kollegialität zwischen einer „menschlichen“ und einer „leistungsbezogenen“ Dimension. Windisch (2017, S. 141) spricht von „Alltagskooperation“ als der „Art und Weise, wie eine Belegschaft bzw. ein Team die gestellten Arbeitsaufgaben gemeinsam bewältigt und welche Arbeitsstile, Routinen, Umgangsformen und Stimmungen dabei vorherrschen [...]. Dabei entwickelt sich eine eingespielte Vertrautheit zwischen den Kolleg/innen und ein Gespür für die Stimmungslage der anderen.“

Diese Alltagskooperation spielt in Krankenhäusern wie in vielen anderen Betrieben eine große Rolle. Sie enthält Elemente von Solidarität, wie die latente Reziprozität und den moralisch verpflichtenden Charakter kollegialen Verhaltens. Von der „Kampf-Solidarität“ unterscheidet sie sich jedoch dadurch, dass die für diese postulierte „Zweipoligkeit“ (Bayertz, 1998, S. 41) fehlt: Laut Bayertz ist „Kampf-Solidarität“ zum einen durch den positiven Bezug auf bestimmte Ziele und Interessen und zum anderen durch das „,charakteristische Engagement gegen einen Gegner“ als „,negativem Bezugspunkt“ gekennzeichnet. Im betrieblichen Kontext ist das in der Regel das Management bzw. der Arbeitgeber. Im Krankenhausalltag geht es hingegen um die kooperative Bewältigung gemeinsamer Aufgaben wie der Patientenversorgung. Wenn sich Beschäftigte in diesem Rahmen füreinander einsetzen und sich kollegial verhalten, ist das nicht mit Solidarität gleichzusetzen. Ich bezeichne dieses Phänomen stattdessen als Kollegialität.

Die Entstehung von Solidarität unter den Beschäftigten wird laut Kröll (2013) durch den Strukturwandel unter neoliberalen Vorzeichen seit Mitte der 1970er-Jahre erschwert. 
Auch Scherr (2013) betont, dass die Solidarisierung von Beschäftigten im postmodernen Kapitalismus voraussetzungsvoll ist. Das gilt umso mehr für die Krankenhäuser, wo zusätzliche Hürden zu überwinden sind. So stehen Arbeitskämpfe in diesem Bereich, die notwendigerweise die Versorgung der Patientinnen und Patienten beeinträchtigen, unter besonderem Rechtfertigungsdruck. Beschäftigte im Gesundheitswesen müssen noch stärker als ihre Kolleg/innen in der Privatindustrie darstellen, dass ihre Ziele ,unter dem Gesichtspunkt der Gerechtigkeit als legitim gelten können“ (Bayertz, 1998, S. 44-45). Und sie müssen die Gefährdung von Menschen ausschließen, zum Beispiel durch sogenannte Notdienst-Vereinbarungen mit dem Arbeitgeber.

Hinzu kommt ein unter Begriffen wie „Zuneigungsgefangenschaft“ (Folbre, 2001) und „Pflegeethos“ (Senghaas-Knobloch, 2008) diskutiertes Dilemma von Beschäftigten im Sozial- und Gesundheitswesen: „Häufig haben sie zu den Kranken [...] persönliche Bindungen aufgebaut. Pflichtbewusstsein und Solidarität ihnen gegenüber führen dazu, dass die Beschäftigten trotz Personalkürzungen und anderer Kostensenkungsmaßnahmen eine gute Versorgung sicherstellen müssen und wollen. Verhandlungen mit Arbeitgebern über Löhne und Arbeitsbedingungen gestalten sich deshalb schwierig und die langfristigen Beziehungen erschweren es, zur Not die Reißleine zu ziehen und die Arbeit niederzulegen oder sich einen neuen Job zu suchen“ (Hipp \& Kelle, 2015, S. 34).

Bischoff (1992, S. 168) kritisierte das „typische Denken“ in der weiblich geprägten Krankenpflege aus feministischer Sicht bereits Anfang der 1990er-Jahre so: „Nicht die Strukturen werden verändert, sondern die einzelne Schwester soll die Strukturen kompensieren. Woher die Probleme kommen, wo die Ursachen der Inhumanität liegen, ob man sie lieber verhütet als ausgleicht, ist für eine solche Krankenpflege keine Frage. Die Gesellschaft wird akzeptiert, wie sie ist: sie ist ja auch gar nicht so wichtig, denn auf den Einzelnen kommt es an, auf die ewige Ich-Du-Beziehung. “

Die meisten Autor/innen kommen aufgrund solcher Rollen(selbst)-Zuschreibungen in Bezug auf die kollektive Interessenartikulation von Pflegekräften zu pessimistischen Perspektiven. So vermutet beispielsweise Rieder (1999, S. 119), „dass es für Krankenschwestern schwierig sein wird, ihre berufliche Identität jenseits der beschriebenen ,Kippbilder (liebend versus herzlos und mütterlich versus genusssüchtig) auszubilden. Dies würde eine Überschreitung oder das Ignorieren der angelegten Identitätsangebote voraussetzen.“

Doch es ist nicht allein die Einstellung zur „Pflege als Berufung“(Voges, 2002), die Loyalität zu den Patientinnen und Patienten, die Pflegekräfte dazu bringt, ihre eigenen Rechte und Arbeitsschutzgesetze zu ignorieren (Becker, 2016). Es ist auch und vor allem die Loyalität zu den Kolleginnen und Kollegen, die Pflegekräfte regelmäßig dazu veranlasst, außerhalb des Dienstplans einzuspringen, Überstunden zu leisten oder gesetzlich vorgeschriebene Pausenzeiten nicht einzuhalten. Der vorliegende Beitrag beschäftigt sich mit Letzterem.

\subsection{Machtressourcen}

Ein theoretischer Bezugspunkt des vorliegenden Beitrags ist der Machtressourcenansatz, der nach den Quellen von Arbeitermacht fragt, wobei der Begriff Arbeiter im Sinne von Lohn- und Gehaltsabhängige verstanden wird. Er „unterstellt ein Interesse mehr oder minder heterogener Arbeiter- und Angestelltengruppen, Asymmetrien in den Austauschbezie- 
hungen von Kapital und Arbeit durch kollektive Mobilisierungen besonderer Machtressourcen zu korrigieren“" (Dörre, 2011, S. 276). Wright (2000, S. 962) und Silver (2005, S. 3044) unterscheiden zwischen struktureller Macht (structural power) und Organisationsmacht (associational power). Erstere erwächst aus der Stellung der Beschäftigten(gruppen) im ökonomischen System und wird in zwei Unterformen unterteilt: Marktmacht (marketplace bargaining power) und Produktionsmacht (workplace bargaining power).

Marktmacht ist primäre Verhandlungsmacht und kann aus verschiedenen Gründen entstehen, zum Beispiel dadurch, dass die Betreffenden über seltene oder besonders nachgefragte Qualifikationen verfügen. Auch ein Fachkräftemangel - wie er für die Gesundheitsberufe und speziell für die Pflege konstatiert wird (SpiegelOnline, 2018) - vermindert die Ersetzbarkeit von Lohnabhängigen und erhöht damit ihre Marktmacht. Der Begriff der Produktionsmacht bezieht sich auf den Produktionsprozess, kann aber auch auf Tätigkeiten im Bereich der Reproduktion bezogen werden. Es ist bemerkenswert, dass es zuletzt insbesondere in Bereichen der Reproduktion - inklusive der Krankenhäuser - zu größeren Arbeitskonflikten gekommen ist (Artus, Birke, Kerber-Clasen \& Menz, 2017). Der Jenaer Arbeitskreis Strategic Unionism (2013, S. 349) führt das unter anderem darauf zurück, dass Reproduktionsarbeit zunehmend warenförmig organisiert ist: „Aus der Kommodifizierung dieser Tätigkeiten erwächst den (oftmals weiblichen) Beschäftigten Reproduktionsmacht. Deren Anwendung in Streiks [...] greift in den Reproduktionsprozess der Ware Arbeitskraft ein.“

Arbeitskonflikte gehen in der Regel mit einem Anwachsen von Organisationsmacht einher. Entscheidendes Kriterium zur Messung von Organisationsmacht ist die „Macht der Zahl“ (Marx, 1972 [1867], S. 196), also der Grad gewerkschaftlicher Organisierung. Allerdings lässt sich die Organisationsmacht nicht allein aus der Zahl der Mitglieder bestimmen, schon allein, weil es sich um eine „rein formale Kategorie“ handelt, die als solche „nichts über die Intensität der Bindung der Mitglieder an die Organisation“ aussagt. „Der Organisationsgrad misst ausschließlich die Fähigkeit einer Organisation zur Beschaffung formalisierter Unterstützungsverpflichtungen“" (Streeck, 1979, S. 72, Hervorhebung im Original). Die Größe von Organisationsmacht hängt ebenso von der Mobilisierungsfähigkeit ab (Müller-Jentsch, 1997, S. 119). Auch wenn diese überwiegend latent bleibt (Dörre, 2011, S. 275), so muss die Fähigkeit zur Mobilisierung doch vorhanden sein - zumindest in den Augen des Verhandlungspartners.

Eine weitere Machtressource ist die institutionelle Macht, die Visser bereits in den 1990er-Jahren konzipiert und in den Gewerkschaftsdiskurs eingeführt hat (Visser, 1995; Ebbinghaus \& Visser, 1999). Als ,sekundäre Machtform“ (Brinkmann \& Nachtwey, 2010, S. 21) entsteht institutionelle Macht ,als Resultat von Aushandlungen und Konflikten, die auf struktureller Macht und Organisationsmacht beruhen. Ihre Besonderheit wurzelt in dem Faktum, dass Institutionen soziale Basiskompromisse über ökonomische Konjunkturen und kurzzeitige Veränderungen gesellschaftlicher Kräfteverhältnisse hinweg festschreiben und teilweise gesetzlich fixieren können. [...] Auf diese Weise präformiert institutionelle Macht die Aushandlungsprozeduren und Handlungsstrategien von kollektiven Akteuren wie Betriebsräten und Gewerkschaften“ (Brinkmann, Choi, Detje, Dörre, Holst, Karakayali \& Schmalstieg, 2008, S. 25).

Zusätzlich zu den genannten Machtquellen konzeptualisiert der Arbeitskreis Strategic Unionism (2013) die gesellschaftliche Macht, die er in Kooperationsmacht und Diskurs- 
macht unterteilt. „Kooperationsmacht meint vor allem, über Netzwerke zu anderen gesellschaftlichen Akteuren zu verfügen und diese für Mobilisierungen und Kampagnen aktivieren bzw. sich an deren Aktionen beteiligen zu können“ (Arbeitskreis Strategic Unionism, 2013, S. 360). Bei der diskursiven Macht, die auf Haug (2009) zurückgeht, kommt hingegen ,zum Ausdruck, erfolgreich in öffentliche Debatten bzw. historisch gegebene hegemoniale Grundstrukturen von Öffentlichkeit intervenieren zu können“(Urban, 2010, S. 444).

Der öffentliche Druck - der ,,vor allem über die Skandalisierung von Ungerechtigkeiten“ (Arbeitskreis Strategic Unionism, 2013, S. 361) entwickelt wird - spielt gerade bei Arbeitskonflikten in Bereichen der öffentlichen Daseinsvorsorge eine große Rolle. Wie bereits erwähnt stehen Arbeitskämpfe in Krankenhäusern unter einem besonderen Rechtfertigungsdruck, da sie die Patientenversorgung einschränken und potenziell gefährden. Zudem ist die direkte ökonomische Wirkung, die Aktionen von Krankenhausbeschäftigten entfalten können, geringer als im Produktionssektor. Die Gewinnung der öffentlichen Meinung, also die Entwicklung von Diskursmacht, ist daher ein bedeutender - wenn nicht der entscheidende - Erfolgsfaktor für Arbeitskämpfe in diesem Bereich. Das wird bei der Analyse der Fallstudien zu berücksichtigen sein.

Macht ist nur als relationale Größe zu begreifen. Wie groß die Macht von Beschäftigten ist, hängt also nicht nur von den ihnen selbst zur Verfügung stehenden Machtquellen ab, sondern auch von den Ressourcen ihres Gegenübers (Brookes, 2018; Schmalz, Ludwig \& Webster 2018). Grundsätzlich ließe sich gegen die Applikation des Machtressourcenansatzes für den vorliegenden Beitrag einwenden, dass dieser bislang eher auf die Verhältnisse in kapitalistischen Wirtschaftsbetrieben gemünzt $\mathrm{zu}$ sein scheint als auf einen weiterhin größtenteils öffentlich oder freigemeinnützig organisierten Bereich der Daseinsvorsorge. Traditionell sind die Arbeitsbeziehungen im Krankenhaus weniger konfliktär und antagonistisch geprägt. Doch die Verhältnisse ändern sich und gleichen sich zusehends an diejenigen in der Industrie an. Der Preiswettbewerb zwingt Klinikleitungen - unabhängig von der Trägerschaft - zu permanenten Rationalisierungsanstrengungen, die wegen des hohen Personalkostenanteils vor allem die Beschäftigten treffen. Klinikleitungen agieren gegenüber Gewerkschaften und Betriebsräten zunehmend wie „normale“ Arbeitgeber und machen auch vor Methoden des „Union Busting“ (Behrens \& Dribbusch, 2014) nicht halt. Das gilt insbesondere für private Betreiber (ver.di, 2018a), doch selbst die landeseigenen Universitätskliniken in Baden-Württemberg bedienten sich Ende 2017 einer einschlägigen Kanzlei, um Streiks zu unterbinden (ver.di, 2017e). Auch in den Krankenhäusern geht es daher zunehmend um machtpolitische Aushandlungsprozesse, die mit dem Machtressourcenansatz untersucht werden können. Allerdings sind nicht alle Machtquellen im untersuchten Feld gleichermaßen relevant, weshalb die gewonnenen Erkenntnisse im Fazit nur in Bezug auf einzelne Aspekte des Ansatzes diskutiert werden.

\section{3 Ökonomisierung und Indirekte Steuerung}

Das Gesundheitsstrukturgesetz von 1993 leitete einen Prozess der Ökonomisierung des Krankenhauswesens ein (Simon, 2016). Zehn Jahre später wurde das Finanzierungssystem grundlegend umgestellt. Das seit 1972 über Jahrzehnte geltende Prinzip der Selbstkostendeckung - die Betriebsausgaben der Krankenhäuser wurden von den Versicherungen unter Maßgabe wirtschaftlichen Handelns vollständig refinanziert - wurde durch ein System von 
Festpreisen (Diagnosis Related Groups, DRG) abgelöst (Rosenbrock \& Gerlinger, 2014). Dieses hat eine „neue Herrschaft der Zahlen“ (Marrs, 2007) im Krankenhaus etabliert.

Das ging mit einer deutlichen Ausweitung des Anteils privater Träger am Krankenhausmarkt einher. So hat sich die Zahl der von Privatkliniken betriebenen Betten seit der Jahrhundertwende auf rund 93.000 (2016) in etwa verdoppelt. Ihre Kapazitäten sind damit zwar immer noch deutlich geringer als die öffentlicher und freigemeinnütziger Betreiber, die Reichweite und der Marktanteil privater Konzerne wächst aber kontinuierlich (Statistisches Bundesamt 2018, S. 15). Diese Entwicklung lässt sich als Verschiebung von Marktgrenzen fassen. Auf Mesoebene, also bezogen auf die Branche insgesamt, werden Marktgrenzen durch Preiswettbewerb und Privatisierung ,auf bislang dekommodifiziertes Terrain“ (Brinkmann, 2011, S. 43) verschoben. Auf betrieblicher Mikroebene findet dies seine Entsprechung in veränderten Formen der Unternehmenssteuerung, die alle Bereiche und Beschäftigten unmittelbar dem Druck des Marktes aussetzen.

Denn die Standardisierung von Krankenhausleistungen ermöglicht es, Kliniken mittels betriebswirtschaftlicher Benchmarks zu vergleichen und in Konkurrenz zueinander zu setzen (Vogd, 2006). Die DRG-Werte richten sich nach Durchschnittskosten, was dazu führt, „dass die Krankenhäuser ihre realen Fallkosten aufgrund eines stipulierten Produktivitätsfortschritts fortwährend absenken und damit die Voraussetzung für eine kostenneutrale Ausweitung der Behandlungstätigkeit schaffen“ (Bode, 2010, S. 194). Dieser „Kellertreppeneffekt“" (Simon, 2013, S. 1785) übt einen permanenten Rationalisierungsdruck aus, auf den die Krankenhäuser in den vergangenen Jahren mit Personalabbau - insbesondere im Bereich der (nicht oder kaum erlösrelevanten) Pflege und der Servicetätigkeiten - sowie mit einer Ausweitung der Fallzahlen reagiert haben. Zugleich haben sich die durchschnittlichen Liegezeiten infolge des medizinischen Fortschritts sowie ökonomischer Anreize sukzessive verkürzt und die Fallschwere hat im Durchschnitt zugenommen. All das führt dazu, dass die Belastung der Pflegekräfte in Deutschland auch im internationalen Vergleich extrem hoch ist: Während eine Pflegefachkraft hierzulande für durchschnittlich 13 Patientinnen und Patienten zuständig ist, sind es in den Niederlanden sieben, in den USA 5,3 (Simon \& Mehmecke, 2017).

Vor diesem Hintergrund basiert die Funktionsfähigkeit der Krankenhäuser zunehmend darauf, dass Pflegekräfte und andere Beschäftigte Leistungen über ihre arbeitsvertraglichen Pflichten hinaus erbringen. Laut DGB-Index Gute Arbeit muss nur weniger als ein Drittel der Beschäftigten in der Regel keine Überstunden leisten, zwei von drei Beschäftigten sind dazu also gezwungen (Roth, 2011). Die Gewerkschaft ver.di hat in einer nicht-wissenschaftlichen Erhebung im Mai 2016 ermittelt, dass die Beschäftigten der Krankenhäuser einen „Überstundenberg“ von insgesamt 35,7 Millionen Stunden vor sich her schieben (ver.di, 2016). Laut einer Online-Befragung von gut 3.500 Pflegekräften aus demselben Jahr ist das Einspringen außerhalb des regulären Dienstplans in der Krankenhauspflege allgegenwärtig: Nur vier Prozent der Pflegekräfte geben an, nie in ihrer Freizeit um die kurzfristige Übernahme von Schichten gebeten zu werden (DBfK, 2016). Hinzu kommen veränderte Aufgabenteilungen zwischen den Berufsgruppen im Krankenhaus. „Rationalisierungsstrategien in Form vertikaler Verlagerungen“ (Becker, 2014, S. 45) zielen darauf ab, Tätigkeiten aufzuspalten und auf geringer qualifizierte (und schlechter entlohnte) Beschäftigtengruppen zu übertragen. Examinierte Pflegekräfte geben zwar Tätigkeiten wie die Essensausgabe an 
Service- und Hilfspersonal ab, weshalb manche examinierte Pflegekräfte dies zunächst begrüßen. Die Übernahme ärztlicher Tätigkeiten führt aber dazu, dass sich die Mehrheit durch die veränderte Aufgabenteilung insgesamt stärker belastet fühlt als zuvor (Bräutigam, Evans, Hilbert \& Öz, 2014, S. 52).

Dass Pflegekräfte im Krankenhaus regelmäßig Aufgaben erfüllen, zu denen sie laut Arbeitsvertrag nicht verpflichtet sind, hat aus Sicht von Gewerkschaften und Interessenvertretungen auch einen potenziell positiven Effekt: Mit „Dienst nach Vorschrift“ kann der laufende Betrieb empfindlich beeinträchtigt werden, was effektive Formen von Widerständigkeit unterhalb des Streiks ermöglicht. Inwieweit Pflegekräfte von dieser Möglichkeit Gebrauch machen, ist Gegenstand dieses Beitrags.

Zugleich ist die Ökonomisierung auch ein Hindernis für einzelbetrieblichen Widerstand. Die einzelnen Kliniken stehen unter hohem Konkurrenzdruck, manchen droht die Insolvenz. Die Leitung dieser Kliniken hat daher oft geringe finanzielle Spielräume. Beschäftigte haben Angst, den eigenen Betrieb und Arbeitsplatz durch Aktionen zu gefährden insbesondere indem sie den Ruf des Krankenhauses schädigen könnten. Das ist wohl der Grund dafür, dass sich die Streiks für Entlastung und auch viele der Ultimaten auf große Universitätskliniken konzentrieren, die als weniger insolvenzgefährdet gelten (BV11, 4).

Nicht nur bei der Einführung marktwirtschaftlicher Mechanismen, auch in Bezug auf die Steuerungsformen von Arbeit manifestieren sich aus der Industrie bekannte Entwicklungen zunehmend auch im Gesundheitswesen. Auch hier etabliert sich ,eine weitgehend enthierarchisierte Struktur der Anweisung und Kontrolle, verbunden mit Formen erweiterter Selbstorganisation und Arbeitsautonomie auf der ausführenden Ebene" (Hirsch-Kreinsen, 2010, S. 453). Zwar war die Selbstorganisation der Stationsteams im Krankenhaus immer schon von großer Bedeutung, sie findet heute aber unter anderen, ökonomisierten Rahmenbedingungen statt. Es gelingt dem Management Marrs (2007) zufolge zwar kaum, die Erlös- und Gewinnoptimierung für die Pflegenden orientierungs- und motivationswirksam zu machen, weil deren ethisch-moralische Ansprüche mit der Ökonomisierung in Konflikt geraten. Dennoch wirkt die „Indirekte Steuerung“ (Peters \& Sauer, 2005; Siemens \& Frenzel, 2016) auch hier: Den Stationsteams wird die Verantwortung dafür übertragen, dass die Patientinnen und Patienten rund um die Uhr versorgt werden - unter Rahmenbedingungen, auf welche die Pflegekräfte keinen Einfluss haben. Das trifft insbesondere auf den Personaleinsatz zu. Dieser gilt als unternehmerische Angelegenheit und entzieht sich daher weitgehend der betrieblichen Mitbestimmung. Eine gesetzliche Personalbemessung, auf die sich Beschäftigte und Interessenvertretungen berufen könnten, gibt es mit Ausnahme weniger Krankenhausbereiche nicht.

Die Kombination beider Entwicklungen - der Ökonomisierung und der Etablierung neuer Steuerungsformen - bildet den Ausgangspunkt und Rahmen für die Veränderungen der Industriellen Beziehungen im Gesundheitswesen.

\section{Falldarstellungen}

Die Strategie der Ultimaten ist Teil eines erweiterten Handlungsrepertoires von Gewerkschaften im Krankenhauswesen. Dazu zählen auch die Streiks für mehr Personal und Entlastung, die zuletzt in mehreren Kliniken stattgefunden haben. Dass die Personalbesetzung 
zum Gegenstand von Tarifauseinandersetzungen gemacht wird, war auch innerhalb der Gewerkschaft ver.di nicht unumstritten, kann aber als punktuell erfolgreiche Ausweitung qualitativer Tarifpolitik interpretiert werden, die zugleich den Druck für eine politische Lösung in Form gesetzlicher Personalvorgaben verstärkt. Die Ultimaten sind Teil dieses Prozesses und können als Vorstufe und Vorbereitung größerer Arbeitskämpfe gelten (BV11, 49; SV01, 16).

Schriftliche Darstellungen der Ultimaten finden sich in lokalen bzw. betrieblichen Publikationen der Gewerkschaft sowie in einer Broschüre der ver.di-Bundesverwaltung (ver.di Region Saar-Trier, o.J.; ver.di Berlin-Brandenburg, o.J.; ver.di, 2015). Die Methode kommt vor allem in drei Regionen zur Anwendung: im Saarland, im Raum Stuttgart sowie in Berlin. Das hängt offenbar vor allem mit den dortigen Akteursstrukturen zusammen. Die Strategie wurde quasi zeitgleich von Aktivist/innen im Saarland und in Stuttgart entwickelt, wenn auch mit gewissen Unterschieden. Im Berliner Universitätsklinikum - das bundesweit als Vorreiter und Beispielgeber der Bewegung für Entlastung gilt - wurde der Ansatz adaptiert und ebenfalls in größerem Maßstab angewandt.

Die Verweigerung freiwilliger Leistungen als Kampfmethode unterhalb des Streiks spielte 2017 zwar auch bei von ver.di bundesweit organisierten Aktionstagen gegen Überlastung eine Rolle. In diesem Rahmen weigerten sich Pflegekräfte vorübergehend, außerhalb des Dienstplans einzuspringen, bestanden auf ihren gesetzlich vorgeschriebenen Pausen oder machten „Dienst nach Vorschrift“ in puncto Hygiene (ver.di, 2017a; ver.di, 2017b; ver.di, 2017c). Anders als die Ultimaten haben die Aktionstage aber eher symbolischen Charakter und dienen der gesellschaftlichen Skandalisierung des Personalmangels - was ver.di außerordentlich gut gelingt. Mit den Ultimaten hingegen versuchen einzelne Pflegeteams, den Arbeitgeber zu konkreten Zugeständnissen zu zwingen, indem sie die dauerhafte Verweigerung freiwilliger Leistungen androhen. Der Beitrag konzentriert sich auf diese Aktionsform, da sie sich potenziell stärker auf das Thema Solidarität auswirkt als einmalige Aktionstage.

Als Fallbeispiele für die vorliegende Untersuchung dienen jeweils zwei Stationen aus Krankenhäusern der drei Regionen. Es handelt sich in allen Fällen um große öffentliche Kliniken, zumeist Universitätskliniken, in denen ver.di vergleichsweise gut aufgestellt ist. Auch hier ist allerdings die Mehrheit der Pflegekräfte in der Regel nicht gewerkschaftlich organisiert. Die Ultimaten sind auch eine Reaktion auf diese Schwäche gewerkschaftlicher Organisationsmacht. Sie können von einzelnen, entschlossenen bzw. gut organisierten Teams begonnen werden, ohne dass die Belegschaft in ihrer Breite bereits arbeitskampffähig sein muss.

Um die Vergleichbarkeit zu gewährleisten, wurde darauf verzichtet, Beispiele aus anderen Einrichtungen oder Beschäftigtengruppen einzubeziehen, die es vereinzelt ebenfalls gibt. Beim Fallbeispiel A handelt es sich um die neurologische Station eines saarländischen Krankenhauses mit insgesamt rund 5.000 Beschäftigten. Die Pflegekräfte der Station riefen im Mai 2015 ein Ultimatum aus: „Wir sind in Sorge sowohl hinsichtlich der pflegerischen Versorgung unserer Patienten als auch in Sorge um unsere eigene Gesundheit"“, hieß es darin. Anfang 2014 hätten der Station noch 26,12 Pflegestellen zur Verfügung gestanden, nun nur noch 18,75. In der Folge hätten sich 3.640 Überstunden angehäuft. „Wir wollen drei zusätzliche Kolleg/innen“, hieß es in dem Text weiter. „Wenn wir bis spätestens 1 . Oktober 
2015 keine neuen Kolleginnen bekommen, dann werden wir nicht länger bereit sein, illegale Tätigkeiten auszuführen.“ Keine Pflegekraft werde dann noch in ihrer Freizeit einspringen, „um kurzfristige Personalausfälle und Unterplanung zu kompensieren“ (ver.di SaarTrier, o.J.). Bei Arbeitsüberlastung werde man nur noch eine Notdokumentation vornehmen. Zudem werde man nicht mehr über die gesetzliche Höchstarbeitszeit von zehn Stunden am Tag hinaus arbeiten. Die große Mehrheit des 26-köpfigen Teams unterzeichnete ein „Teamversprechen“, das ab 20 Unterschriften in Kraft treten und nicht veröffentlicht werden sollte.

Die Pflegekräfte dokumentierten das Ultimatum gegenüber Patient/innen und anderen Beschäftigten mit einem von ver.di produzierten Button mit der Aufschrift „NC 02 Neuro? Logisch! Am 1.10. ist Schluss.“ Die Geschäftsleitung sprach in einem Schreiben von „einem klaren Erpressungsversuch“ (ebenda) und lehnte Verbesserungen zunächst kategorisch ab. Sie untersagte den Beschäftigten, die Buttons bei der Arbeit zu tragen. Ver.di reagierte kreativ: Die Gewerkschaft verteilte statt der Buttons einfache Wäscheklammern - nicht nur auf der betreffenden Station, sondern im ganzen Haus. Schon bald war die Wäscheklammer allgegenwärtig. Auch in anderen Krankenhäusern der Region trugen Pflegekräfte diese „Klammer der Solidarität“ (SV01, 36).

Im Team selbst stieg der gewerkschaftliche Organisationsgrad im Verlauf der Auseinandersetzung auf 65 Prozent. Zwei Wochen vor Ablauf des Ultimatums kam es zur Einigung, nachdem sich der gesamte Klinikvorstand mit dem Stationsteam getroffen hatte: Die Geschäftsleitung sagte zu, drei Pflegekräfte zusätzlich einzustellen und die Zahl der Betten um vier zu reduzieren. Zudem sollten ein Springer-Nachtdienst für das Neurologie-Zentrum eingerichtet und der Station ein weiteres Handy sowie ein Laptop zur Verfügung gestellt werden (SB12).

$\mathrm{Zu}$ denjenigen, die durch das Beispiel ermutigt wurden, gehörten die Pflegekräfte einer onkologischen Station des gleichen Krankenhauses, die das Fallbeispiel B darstellt. Sie kündigten im Oktober 2017 an, ab dem 1. Februar 2018 freiwillige Leistungen zu unterlassen, falls nicht drei zusätzliche Vollzeitstellen examinierter Pflegekräfte eingerichtet würden. Den Anstoß dafür gab ausgerechnet der Arbeitgeber: Im Frühjahr hatte ver.di das Klinikum und andere Landeseinrichtungen in Zusammenhang mit der Tarifrunde der Länder zu Warnstreiks aufgerufen. Die Klinikleitung bestand bei den Verhandlungen über eine Notdienstvereinbarung darauf, die onkologische Station im Früh-, Spät- und Nachtdienst mit fünf, vier bzw. zwei examinierten Pflegekräften zu besetzen. „Wir mussten Kolleginnen, die streiken wollten, wieder auf die Station schicken, um die Mindestbesetzung zu erfüllen“ (Supe, 2018, S. 5), so der zuständige ver.di-Sekretär. Doch in den folgenden Monaten wurde diese, von der Klinikleitung selbst ins Spiel gebrachte Mindestbesetzung immer wieder unterschritten. Das veranlasste das Team zum Ultimatum, das von 17 Pflegekräften (bei 18,5 Vollzeitstellen) durch ihre Unterschrift unter das „Teamversprechen“ unterstützt wurde.

Im Rahmen eines „Eskalationsplans“ steigerten sie sukzessive den Druck auf die Klinikleitung: Ab dem 1. Dezember 2017 zählten sie die Tage bis zum Ablauf des Ultimatums mit Aufklebern auf ihren Arbeitskitteln herunter. Kurz vor Weihnachten organisierte ver.di eine Pressekonferenz, die große öffentliche Aufmerksamkeit erregte. Diese wurde noch dadurch gesteigert, dass die Gewerkschaft Anfang Januar 2018 Pflegekräfte im Saarland 
dazu aufrief, sich am 1. Februar zum Noteinsatz auf der Station zu melden. Geplant war zudem eine 24-stündige Mahnwache auf einem öffentlichen Platz. Dazu kam es dann aber nicht mehr, weil die Klinikleitung zuvor Zugeständnisse machte. Die 15 Tage vor Ablauf des Ultimatums erzielte Einigung sah vor, die Zahl der Pflegefachkräfte von 18,5 auf 21 zu steigern und zwei weitere Medizinische Fachangestellte einzustellen. Zudem sollten strukturelle Veränderungen vorgenommen und die Wirkung der Maßnahmen zwei Monate später evaluiert werden. Zu Beginn hätten die Pflegekräfte ihre Angst überwinden müssen, so eine der Beteiligten. Doch das Ultimatum habe das Team „zusammengeschweißt“ (SB02, 11, 49; ver.di Saar-Trier, o.J.).

Die Ultimaten in der Region Stuttgart unterscheiden sich in einigen Aspekten von denen im Saarland. So wenden sich die Pflegekräfte zumeist nicht an eine breitere Öffentlichkeit und an die Medien. So auch in Fallbeispiel $C$, das sich auch in seiner Dramaturgie deutlich von den beiden erstgenannten Fällen unterscheidet. Nachdem sie ihre Überlastung beinahe täglich in sogenannten Überlastungs- oder Gefährdungsanzeigen (ver.di b+b, 2017) dokumentiert hatten, ohne dass die Klinikleitung reagierte, kündigten die Pflegekräfte der Station in einem Brief an die Zentrumsleitung an, ab sofort nicht mehr außerhalb des Dienstplans einzuspringen. Angesichts der extrem hohen Belastung habe es ,eine große Geschlossenheit im Team“ gegeben (SWB06). Das Vorgehen der Pflegekräfte zeigte unmittelbar Wirkung: An einem Wochenende musste die Station kurzfristig geschlossen werden, da kein Nachtdienst zur Verfügung stand. Danach wurde die Bettenkapazität dauerhaft um ein Drittel reduziert, obwohl die Klinikleitung Bettenschließungen zuvor kategorisch ausgeschlossen hatte. Dieser Erfolg habe sich auf das Alltagsbewusstsein positiv ausgewirkt, so eine ver.di-Vertrauensfrau. „Die Kolleginnen jammern nicht mehr nur, sondern setzen sich für ihre Interessen ein“ (ver.di, 2015, S. 30).

Die dauerhafte Sperrung von Betten forderten auch die Pflegekräfte einer Station eines nahegelegenen Krankenhauses im Juni 2015, in Fallbeispiel D. Andernfalls werde man nicht mehr außerhalb des Dienstplans einspringen. Doch anders als in den anderen untersuchten Fällen blieb die Klinikleitung hart. Sie nannte die Forderungen gegenüber der Presse ,absolut unerfüllbar“ und unterstellte ver.di eine aggressive Kampagne, die der Klinik schade (Braun, 2015). Die zuständige Gewerkschaftssekretärin verwies hingegen darauf, dass die Pflegekräfte lediglich angekündigt hätten, eine freiwillige Leistung künftig nicht mehr zu erbringen - ihren Dienstplan erfüllten sie weiterhin. Wenn dies schon zu einem Zusammenbruch des Systems führe, sei das auf ein Organisationsversagen des Arbeitgebers zurückzuführen.

Der Arbeitgeber ließ das Datum verstreichen, ohne auf die Forderung der Pflegekräfte einzugehen. Ein ver.di-Sekretär berichtet: „Im Endeffekt wurden nachher die Zimmer gesperrt und also die Betten gesperrt, aber das Team hat schon richtig viel Druck gekriegt“" (SWV03, 28). Insbesondere die Stationsleitung, die den Zielen des Teams gegenüber positiv eingestellt war, sei massiv unter Druck gesetzt worden: „Die Stationsleitung war völlig fertig mit den Nerven und das hat das Team natürlich mitgekriegt und das ist ein psychischer Druck. Man will die Stationsleitung ja nicht verheizen und fertig machen, sondern mit der arbeiten sie eng zusammen, die mögen sie auch“ (SWV03, 30). Druck gab es auch von den Pflegekräften der Nachbarstation, die einspringen mussten, als das Ultimatum in die Tat umgesetzt wurde: „Insofern wurden halt die Teams gegeneinander ausgespielt und das 
Team, was das Ultimatum umgesetzt hat, hatte einen ganz schlechten Ruf im Haus. [...] Das wirkt bis heute nach [...] und das ist natürlich was, was man in Zukunft tatsächlich verhindern muss, dass man da eher die Solidarität nicht nur im Team hinkriegt, sondern wirklich auch mit den anderen Teams drumherum" (SWV03, 30).

Obwohl die Klinikleitung letztlich nicht umhin kam, Betten zu sperren und damit die Forderung der Pflegekräfte zu erfüllen, führte dieses Ultimatum nicht dazu, andere Teams zu ähnlichen Aktionen zu motivieren - im Gegenteil. „Das war ja auch Sinn und Zweck von der Geschäftsführung, dass dann eben nicht Nachahmerstationen direkt danach kommen, weil niemand will so schlecht dastehen wie dieses Team dann" (SWV03, 32). Die örtlichen ver.di-Aktiven zogen aus dieser Erfahrung die Schlussfolgerung, möglichst nicht mehr mit nur einem Team in eine solche Auseinandersetzung zu gehen.

Am Berliner Uniklinikum kommen Ultimaten, hier „Notrufe“ genannt, seit dem Frühjahr 2013 immer wieder zur Anwendung. Den Anfang machten zwei benachbarte Nephrologie-Stationen, die einen „Notruf“ an die Geschäftsleitung, den Personalrat und die Öffentlichkeit formulierten und die Schließung von jeweils zehn Betten forderten. „Wir haben vorgerechnet, dass der Betrieb ohne ein systematische Überplanung nicht aufrecht zu erhalten ist", berichtet ein Krankenpfleger (CB09). Bei diesem Fallbeispiel E drohten die Pflegekräfte nicht nur damit, außerhalb des Dienstplans nicht mehr einzuspringen, sondern auch, weitere freiwillige Leistungen zu verweigern. Das betraf Tätigkeiten als Hygiene-, Geräte-, Dokumentations- und Arbeitssicherheitsbeauftragte sowie die Praxisanleitung von Auszubildenden. Auch ärztliche Tätigkeiten - vor allem Blutentnahmen sowie die Applikation von Medikamenten und Infusionslösungen - sollten in unterbesetzten Schichten ,zurückdelegiert“ werden. „Die ärztlichen Kollegen sind oft gar nicht mehr in der Lage, Dialysegeräte zu bedienen oder Infusionen richtig zu legen“, erklärt der Kernaktivist der Station. „Daher hat die Verweigerung dieser Arbeiten ein enormes Druckpotenzial.“ Zudem steige das Selbstbewusstsein der Beschäftigten: „Allen wird klar: Ohne die Pflege läuft es nicht.“ Ver.di machte das Ultimatum öffentlich und informierte die Belegschaft mit 3.000 Flugblättern. Die pflegerische Zentrumsleitung verhandelte mit den vom Team delegierten Sprecher/innen und bot für die eine Station zwei, für die andere drei zusätzliche Stellen an. Auf dieser Basis wurde der Konflikt beigelegt (CB09; ver.di Berlin-Brandenburg, o.J.; ver.di, 2015, S. 25-26).

Obwohl die Strategie der Ultimaten am Berliner Großklinikum seit Jahren flächendeckend angewandt wird, mussten die Pflegekräfte ihre Drohungen noch nie umsetzen. Wie im Saarland machte das Management stets zuvor Zugeständnisse. In einigen Fällen wurden die Auseinandersetzungen beigelegt, bevor diese öffentlich wurden. So auch in Fallbeispiel $F$, einer Stroke Unit zur Behandlung von Schlaganfall-Patient/innen. Einige Jahre zuvor wurde dort die Monitorüberwachung eingeführt. Erst waren es vier, dann sechs Monitore, die permanent beobachtet werden mussten. „Diese anspruchsvolle Aufgabe wurde uns zusätzlich auferlegt, ohne dass wir dafür auch nur einen Cent mehr Geld bekommen haben“, sagt eine Krankenpflegerin über den Anlass des Ultimatums. Die 22 Pflegekräfte forderten monatlich 150 Euro mehr Geld. Andernfalls werde das Team Aufgaben als Beauftragte, ärztliche Tätigkeiten und Flex-Dienste künftig ablehnen, hieß es in ihrem „Notruf“. Voraussetzung seien ein „guter Teamzusammenhalt“ und ein hoher gewerkschaftlicher Organisationsgrad gewesen, so eine Aktivistin (CB10, 26). 
Das Management reagierte schnell: Es bot eine Zulage von monatlich 75 Euro an. Obwohl das nur die Hälfte der geforderten Summe war, habe das Team dies ,in einem demokratischen Abstimmungsprozess angenommen“ (CB10, 16), so eine Krankenpflegerin, die die positive Wirkung der „Notrufe“ auf die Solidarität im Team betont: „Teams, die eine solche Notruf-Aktion zusammen durchgestanden haben, sind in der Regel auch im Arbeitskampf eine Bank“ (CB10; ver.di, 2015, S. 28-29).

\section{$4 \quad$ Prozesse und Akteure}

Entwicklung von Kampfsolidarität im Team. Ein Schlüssel bei der Formierung widerständiger Solidarität im Zuge der Ultimaten ist das sogenannte Teamversprechen. Es muss von einem zuvor festgelegten Teil des Teams - in einem Fall sind das mindestens 90 Prozent der Pflegekräfte - unterzeichnet werden. Erst wenn genügend Unterschriften zusammen sind, wird das Ultimatum offiziell verkündet, die Namen werden nicht veröffentlicht. Durch das Teamversprechen wird die, so formuliert es ein Personalratsmitglied, im Klinikalltag ,,auf den Kopf gestellte“ Solidarität ,wieder auf die Füße gestellt“ (SWB04, 30-32). Die Vorzeichen ändern sich: Nun gilt es nicht mehr als solidarisch, außerhalb des Dienstplans einzuspringen, sondern im Gegenteil: Wer dann noch einspringt, „hat in dem Team nichts mehr zu suchen, er stellt sich dann selber außerhalb des Teams“ (SV01, 39). Alle Beteiligten beschreiben dieses formalisierte Versprechen mit seinem „rituellen, symbolischen Charakter" (CB10, 24) als ein zentrales Instrument zur Herstellung widerständiger Solidarität im Team (SV01, 32; BV11, 35-37; SWB04, 34).

Hinzu kommen weitere Erfahrungen, die die Pflegekräfte während des Ultimatums machen. Sie artikulieren sich gegenüber leitenden Vorgesetzten, überwinden Ängste, zum Beispiel im Umgang mit Medien, und treten sichtbar als kollektive Akteure in Erscheinung, beispielsweise durch Anstecker/Aufkleber oder Aktionen in der Öffentlichkeit. All das befördert den Prozess der Entwicklung von Alltagskollegialität zu „Kampf-Solidarität“, der in Abschnitt 5 ausführlicher beschrieben wird (SV01, 9, 35-37; SB02, 91).

Die Rolle anderer Pflegeteams. Die Solidarisierung anderer Stationsteams im Krankenhaus gilt den Beteiligten als entscheidender Erfolgsfaktor für die Ultimaten (SV01, 38). Sie wird dadurch erschwert, dass , die Teams untereinander [...] sich eigentlich gar nicht kennen, sondern sich immer nur auf das eigene Team beziehen“ (SWV03, 32). In einem Fallbeispiel habe der Arbeitgeber dies gezielt ausgenutzt, um „Stimmungen“ gegen das Team der Ultimatum-Station zu erzeugen, was letztlich zum Scheitern des Ultimatums geführt habe (SWV03, 36; SV01, 37, 90-94).

Die Rolle von Ärzt/innen, Patient/innen und anderen Berufsgruppen. Die Ultimaten sind eine Aktionsform der Pflegeteams. Andere Berufsgruppen spielen in der Regel keine Rolle. Relevant ist lediglich, welche Haltung die Ärztinnen und Ärzte einnehmen, mit denen Berührungspunkte und latente Konfliktlinien bestehen - beispielsweise wenn Pflegekräfte ärztliche Tätigkeiten an die Mediziner/innen ,zurückdelegieren“. Die Haltung von Ärzt/innen gegenüber den Aktionen ist den Interviews zufolge unterschiedlich und hängt von der individuellen Einstellung, vor allem aber von der Verortung innerhalb der ärztli- 
chen Hierarchie ab. Während sich die auf der betreffenden Station tätigen Assistenzärzte zumeist solidarisieren, lehnen Ober- und vor allem Chefärzte die Aktionen oft entschieden ab. Die Ärzteorganisation Marburger Bund spielt in den Auseinandersetzungen keine wahrnehmbare Rolle. Bei Streiks des Pflegepersonals hat sie in einigen Fällen ihre Solidarität bekundet (SV01, 98, 105; CB10, 66-74; BV11, 32; SWV03, 50; SWB04, 13-16; MB1). Wenn es von Patientinnen und Patienten Reaktionen gibt, sind diese den Pflegekräften zufolge meistens positiv (SB02, 93; SV01, 114; SWB04, 52).

Die Rolle vorgesetzter Pflegekräfte. Pflegedienstleitungen und -direktoren sind nach Wahrnehmung der beteiligten Pflegekräfte Teil des Managements. Sie gelten als Verhandlungs- und Konfliktpartner, die konfrontativer oder konzilianter auftreten können (SWB04, 38; SWV03, 28-30; SB02, 29-31, 39-43). Ganz anders wahrgenommen wird die Stationsleitung. Sie ist in der Regel gut in das Team integriert, steht aber aufgrund ihrer hervorgehobenen Stellung unter dem Druck, den Betrieb am Laufen zu halten. Wenn sich eine integrierte Stationsleitung gegen das Ultimatum stellt, ist dessen Umsetzung nach Einschätzung der Gewerkschaftsaktiven fast unmöglich. Auf den betreffenden Stationen hätten diese Leitungskräfte die Ultimaten aber zumeist passiv unterstützt und ,weggehört“ (SV01, 135-137; BV11, 8; SWV03, 30; SWB04, 40).

Die Rolle der betrieblichen Interessenvertretung. Betriebs- und Personalräte haben sich zu den untersuchten Ultimaten unterschiedlich verhalten. Während Personalratsmitglieder, die zugleich ver.di-Vertrauensleute waren, in einigen Fällen als „Ideengeber, Koordinatoren, Helfer in Problemsituationen“ (SWB04, 36; SWV03, 70) fungierten, verhielten sich andere passiv (SWV03, 76). Ein ver.di-Sekretär berichtet, das Verhältnis zu den Interessenvertretungen sei „,manchmal schwierig, [...] weil die oftmals abwartend dabeistehen und auch ein Ultimatum als einen persönlichen Angriff empfinden. Es gibt auch sowas wie Eifersucht" (SV01, 91-93).

Die Rolle der Gewerkschaft. Ver.di spielte bei allen untersuchten Ultimaten eine entscheidende Rolle, allerdings nicht immer in Person eines hauptamtlichen Sekretärs. In einigen Krankenhäusern waren es eher betriebliche Vertrauensleute, die dem Team zur Seite standen (SWB04, 54). Im Saarland ist hingegen der zuständige ver.di-Sekretär bei allen U1timaten stark engagiert, verteilt „Schutzbriefe“ und „Notfallkarten“, mit denen er rund um die Uhr erreichbar ist (SV01, 35, 42). „Wichtig ist, immer wieder vor Ort zu sein, die Kollegen nicht allein zu lassen“, betont dieser Hauptamtliche. ,Weil natürlich [...] kommen die Chefs, der Abteilungsleiter, und versuchen dort rein zu brechen. Und natürlich fangen Kollegen an zu zweifeln. Und das muss man im Team immer wieder auffangen, das ist ganz wichtig“" (SV01, 115).

Er betont - ebenso wie alle anderen haupt- und ehrenamtlichen Gewerkschafter/innen, die in die Ultimaten involviert sind - seinen ,Anspruch [...], keine Stellvertreterpolitik zu machen“. Das heiße aber „,nicht, wie manche Kollegen meinen, dass man nicht die Prozesse führen darf. [...] Sondern ganz im Gegenteil. Das verlangt von dir eine viel intensivere Arbeit" (SV01, 128). Es handelt sich also keineswegs um einen führungslosen Prozess, in dem die Pflegekräfte völlig eigenständig agieren. Alle Beteiligten betonen jedoch, dass das Pflegeteam selbst entscheidet, welche Angebote es annimmt oder zurückweist (ver.di, 2015, 8; SV01, 46; ver.di Region Saar-Trier; SWV03, 54-56; SWB04, 8, 36). In einem Fall berichtet 
ein ver.di-Sekretär davon, dass sich das Team trotz seiner Warnungen für eine „Aktionspause“ entschied, nachdem das Management Gespräche angeboten hatte: „Und ich war von ver.di aus bei dem Treff und habe eben gesagt: ,Hier, damit werdet ihr das Ziel nicht erreichen!' [...] So ist es auch gekommen, [...] diese Gespräche haben alle gar nichts gebracht [...]. Und es ist aber leider dann auf ver.di zurückgefallen dort, weil ,mit den ver.diAktionen hat man nichts erreicht' [...]. Das hat für mich die Lernerfahrung, nächstes Mal viel klarer zu sagen: ,Wenn ihr die Entscheidung jetzt trefft, dann müsst ihr mit den Konsequenzen nachher leben [...] und es dann nicht ver.di sozusagen in die Schuhe schieben“" (SWV03, 56).

Öffentlichkeit und Legitimation. Wie stark die Auseinandersetzungen in die Öffentlichkeit getragen werden, ist in den untersuchten Regionen unterschiedlich. Während ver.di im Saarland mit einem „Eskalationsplan“ (SV01, 34) gezielt versucht, sukzessive den öffentlichen Druck auf die Klinikleitung zu steigern, werden die meisten Ultimaten im Stuttgarter Raum nicht in die überbetriebliche Öffentlichkeit getragen. Am Berliner Universitätsklinikum kommt ein abgestuftes Vorgehen zur Anwendung: Zunächst wird versucht, die Klinikleitung innerbetrieblich zu Zugeständnissen zu bewegen, wenn das nicht reicht, werden die Fälle publiziert (SB02, 69-75, 91; SV01, 35, 73-78; CB10, 29-38, 44; SWB04, 8, 46). Doch auch in den Fällen, die nicht veröffentlicht werden, ist die Drohung damit ein wichtiger Faktor für Erfolge. Es ist also von hoher Bedeutung, ob die Pflegekräfte dazu bereit und in der Lage sind, Diskursmacht zu entwickeln. Ver.di betont bei den Streiks und Ultimaten stets, dass es den Beschäftigten nicht nur um ihre eigenen Interessen gehe, sondern auch um das Gemeinwohl. Das kommt auch in den Parolen zum Ausdruck, die die Gewerkschaft häufig verwendet: „Mehr von uns ist besser für alle!“”; „Nicht der Streik gefährdet die Patienten, sondern der Normalzustand!“. Die Arbeitgeber würden die Verantwortung für Einschränkungen bei der Versorgung tragen. Durch die langfristige Ankündigung der Aktionen - ein markanter Unterschied zu Arbeitskämpfen in anderen Branchen, bei denen in der Regel sehr kurzfristig angekündigt wird, welche Bereiche betroffen sind - habe das Management genügend Zeit, sich auf die Situation vorzubereiten und zum Beispiel die Zahl der Neuaufnahmen zu reduzieren. Auf diese Weise versucht die Gewerkschaft, der besonderen ethischen Problematik von Arbeitskämpfen im Krankenhaus zu begegnen und sowohl die Streikbereitschaft als auch die öffentliche Unterstützung zu erhöhen (SV01, 96; ver.di, 2018b; ver.di, 2018c; ver.di Berlin-Brandenburg, 18).

\section{Diskussion}

\subsection{Von disziplinierender Kollegialität im Klinikalltag...}

Die neuen Steuerungsformen von Arbeit im Krankenhaus haben aus Sicht betrieblicher Interessenvertreter/innen eine problematische, zumindest aber ambivalente Wirkung: „Die, sagen wir mal Verflachung der Hierarchie, also [dass es] ganz wenige Häuptlinge praktisch gibt, was ja erst mal klingt wie: ,Ah, erster Erfolg, kann man autonom seine Arbeit selber bestimmen. ' Aber dann im Endeffekt eben bewirkt hat, dass die ganze Verantwortung nach unten verlagert wurde und die Rückübertragung der Verantwortung nicht mehr möglich 
war, weil die da oben so ausgedünnt worden sind, nicht mehr erreichbar waren. Und das bewirkt dann natürlich, dass man [...] auf seine Rechte verzichtet und [...] das alles praktisch selber gewährleisten muss, was läuft [...] - was eigentlich die Aufgabe von Chefs ist" (SWB04, 32).

In dieser Situation wird das Pflege-Team zur „Schicksalsgemeinschaft“, die ,die Situation irgendwie zu bewältigen“ (SWB04, 2) hat: „Die Teams sagen: ,Ja, die Welt ist schlecht drumherum, aber in der schlechten Welt sind wir noch die Besten. ' Ja? ,Wir versuchen das zu regeln. ' Und diese, also positiven Ansätze werden genommen, dass es funktioniert. Damit erreichen die Kollegen natürlich gar nichts. [...] Die einzige Möglichkeit, wie sie sich [...] retten können aus diesem Teufelskreis, ist, indem sie dann krank werden, oder einen Krankenschein machen. Wie auch immer. Weil das das Einzige ist, was akzeptiert wird, [...] innerhalb des Teams“ (SV01, 18-19).

Auf diese Weise wird „,der Teamzusammenhalt zur Selbstausbeutung genutzt [...], oder zur gegenseitigen Selbstausbeutung“ (BV11, 53). Für die Führungskräfte bedeutet diese Form der Steuerung eine Entlastung, denn ,erstens hat man halt nicht so viel Arbeit damit und zweitens zieht man die Kritik nicht auf sich. Und es funktioniert eben auch sehr gut, dass die Leute dann ihren Kolleginnen und Kollegen zu Liebe das dann machen, was sie vielleicht gegenüber einer Vorgesetzten, einem Vorgesetzten irgendwann dann auch mal sagen würden: ,Ne, ich kann jetzt nicht ans Telefon gehen.“" (BV11, 4). Die Beschäftigten stehen hingegen vor einem Dilemma: „Und dann stellt sich halt immer mehr die Frage: [...] Wo mache ich Abstriche? Am Patienten [oder] verzichte hier auf meine Pause und kann vielleicht doch ein bisschen länger machen und springe ich ein im Frei und stoße meiner Familie vor den Kopf oder provoziere ich unter Umständen, dass meine Kollegin statt für zehn Patienten plötzlich für 15 Patienten zuständig ist [...]? Und das ergibt sich dann halt in der alltäglichen Solidarität, um den Beruf irgendwie ausüben zu können, mit Rahmenbedingungen auf die man halt keinen Einfluss hat" (SWB04, 2).

Dass das „Verantwortungsgefühl gegenüber den Patientinnen und Patienten“ (BV11, 10) Pflegekräfte dazu bringt, ihre eigenen Rechte zu ignorieren und ihre Gesundheit aufs Spiel zu setzen, wird in der Literatur breit diskutiert. Dieses Phänomen findet sich auch in den Interviews wieder. Doch eine mindestens ebenso starke Wirkung scheint das Bedürfnis zu haben, „die Kollegen [...] nicht im Stich zu lassen“ (CB10, 5-10). Ein ver.di-Sekretär benennt beide Aspekte als Motivation, außerhalb des Dienstplans einzuspringen: „[Der Pfleger] kommt aus dem Frei, weil er keine andere Lösung sieht, weil er sich erinnert, dass der Kollege auch aus dem Frei gekommen ist als er krank war und [...] weil er natürlich auch konkret den kranken Menschen sieht, den er nicht allein lassen will“ (SV01, 6). Ein anderer Gewerkschafter betont, die Pflegekräfte ,sehen die direkten Kollegen dann vor sich, wie sie dann alleine [...] arbeiten müssen und wissen am eigenen Leib, was das bedeutet" (SWV03, 8).

Hier wird deutlich, dass dieses Verhalten auf Reziprozität beruht: Die Pflegekraft verknüpft ihre Bereitschaft zu zusätzlichem Engagement mit der Erwartung, dass die Kolleg/innen sich ebenso verhalten. Dementsprechend gelten diejenigen, die auf ihrem Recht bestehen, als „egoistisch“ (BV11, 12) und sind ,im Team isoliert“ (SV01, 5) bzw. ,im sozialen Abseits“ (SWV04, 6). So werde „die Solidarität auf den Kopf gestellt“, meint ein Krankenpfleger, ,weil diese Solidarität eben bedeutet, dass man den ganzen Mist mitmacht, 
auf die Rechte verzichtet“ (SWB04, 32, 30). Auch eine hauptamtliche Gewerkschafterin betont: „Sich gegenseitig im Leid beizuspringen und das Leid zu verlängern, ist zumindest eine fragwürdige Form von Solidarität. Weil Solidarität heißt für mich eigentlich, sich gegenseitig dabei beizustehen, für eine Verbesserung zu kämpfen. Also für mich ist Solidarität auch ein Kampfbegriff" (BV11, 16).

Dieses Verständnis von Solidarität als „Kampfsolidarität“ wird in den Interviews von allen Aktivist/innen grundsätzlich geteilt (CB10, 12; SWV03, 10; SWV04, 4). Ein ver.diSekretär argumentiert allerdings, dass die Kollegialität im betrieblichen Alltag „ein solidarisches Element" hat, das die Gewerkschaft „nicht etwa bekämpfen, sondern stabilisieren“ müsse (SV01, 16-17). In der Vergangenheit habe ver.di das nicht gemacht. Mit der Parole „Dein Frei gehört dir" forderte sie Pflegekräfte individuell auf, ihre Rechte wahrzunehmen und nicht mehr außerhalb des Dienstplans einzuspringen - mit geringem Erfolg (SWV03, 12; BV11, 14). Jetzt versuche ver.di, „von dem Individuellen hin zum Kollektiv [zu] kommen“ (SV01, 10): „Ich war Stationsleiter und ver.di hat sich ausgedacht ,Mein Frei gehört mir.' Also [...] stand ich plötzlich da als Stationsleiter und hab gedacht: ,Scheiße! Jetzt kriege ich Schwierigkeiten damit, [...] einen gewerkschaftlichen Auftrag zu erfüllen. Warum ist denn das so schwer?' Weil ich natürlich den Dienstplan selber organisieren musste und bei Ausfällen selbstverständlich auch Leute angerufen habe: ,Du musst jetzt arbeiten kommen. ' $[\ldots]$ Und da ist mir dann eine Idee gekommen, dass das ganze Problem eben gar nicht lösbar ist, indem ich das nur individuell betrachte und dem Mitglied sage: ,Du musst Nein sagen.', sondern ich brauche kollektive Prozesse. Ich muss also das gesamte Team ansprechen“"(SV01, 4-6).

\section{$5.2 \quad \ldots$ zu widerständiger Solidarität}

Aus Sicht der betrieblichen Akteure hat die Ökonomisierung neben den dargestellten problematischen Auswirkungen auch zur Folge, dass Krankenhausbeschäftigte - nicht nur Ärztinnen und Ärzte, sondern auch Pflegekräfte und teilweise sogar Mitarbeiter/innen der Servicebereiche - Zugriff auf zuvor verschlossene Machtquellen erhalten, namentlich Produktionsmacht: „Wenn ich das Krankenhaus zu einer Fabrik mache, dann müssen die Beschäftigten des Krankenhauses auch lernen, sich wie Arbeiter zu benehmen, [...] zu verstehen, dass ihre große Stärke darin besteht, ihre Ware Arbeitskraft $[\ldots]$ verweigern zu können und damit etwas auszulösen - nämlich fehlende Einnahmen. Das stellt eine Macht dar. Diese Macht hatten sie in den früheren Jahren eben so gesehen nicht" (SV01, 139).

Eine Gewerkschaftsfunktionärin betont, die Beschäftigten sollten sich dieser für den Krankenhausbereich neuen Machtressource bewusst werden: „Wenn ein Krankenhaus nach dem Gewinnprinzip letzten Endes funktioniert, oder nach einem Erlösprinzip, und du sorgst dafür, dass die Erlöse wegfallen, dann hat deine Krankenhausleitung ein Problem, dann richtest du ja einen realwirtschaftlichen Schaden an. Das war bei dem Selbstkostendeckungsprinzip nicht so. [...] Das ist vielen in der Belegschaft noch nicht so klar, was sie da eigentlich für ein Machtmittel in der Hand haben“ (BV11, 55).

Ein anderer ver.di-Sekretär argumentiert allerdings, durch die Verweigerung freiwilliger Leistungen auf einzelnen Stationen werde kein ernsthafter ökonomischer Druck erzeugt (SWV03, 64). Das sei erst bei Streiks der Fall, in deren Folge Betten oder ganze Stationen vorübergehend geschlossen werden müssen. Die beiden Aktionsformen - Ultimaten sowie Betten- und Stationsschließungsstreiks - können indes als Teil einer veränderten Gesamt- 
strategie der Gewerkschaft im Gesundheitswesen interpretiert werden. Denn auch bei der neuen Streikform geht es darum, ,kollektive Prozesse in Gang zu setzen“ (SV01, 82): Während ver.di in vergangenen Arbeitskämpfen an einzelne Beschäftigte appellierte, die Arbeit niederzulegen, geht der Appell jetzt an die Teams. Sie sollen sich kollektiv und im Vorfeld darüber verständigen, wie stark sie sich an der Arbeitsniederlegung beteiligen wollen. Wenn beispielsweise die Hälfte der Pflegekräfte in Streik treten will, fordert ver.di den Arbeitgeber auf, jedes zweite Bett in dieser Zeit nicht zu belegen. Zum Teil werden ganze Stationen zum Streik ,,angemeldet“ - so frühzeitig, dass die Klinikleitung sie leer räumen kann. Zuerst entwickelt wurde diese neue Streikform 2011 am Berliner Universitätsklinikum. Sie löst das Dilemma der Pflegekräfte teilweise auf, sich zwischen Streik und Patientenversorgung entscheiden zu müssen. „Denn: Wo keine Patient/innen sind, kann niemand zu Schaden kommen“ (ver.di Berlin-Brandenburg, o.J., S. 13). Mit dem Argument, das Streikrecht der Pflegekräfte könne letztlich nur so ungehindert in Anspruch genommen werden, versucht ver.di, vor Streiks entsprechende Notdienstvereinbarungen mit den Kliniken zu schließen (ver.di Berlin-Brandenburg, o.J., S. 18-20).

Die ver.di-Aktiven sehen die Ultimaten dabei auch als Vorbereitung eines Betten- und Stationsschließungsstreiks. Sie haben ,den Effekt, dass die Beteiligten die Erfahrung machen: Nur wenn man sich gemeinsam wehrt, kann man etwas erreichen. Je mehr Teams diese Erfahrung machen, desto breiter wird die Bewegung für Entlastung und umso mehr können wir erreichen“ (SV01b, 16). Eine Gewerkschafterin betont: „Wenn man Stationen hat, die schon einmal durch so ein Ultimatum gegangen sind, [...] wissen [sie], wie der Teamzusammenhalt funktioniert und wie man den auch in schwierigen Situationen aufrecht erhält und [sind] deswegen wahrscheinlich auch gute Streikstationen, das würde ich auf jeden Fall sagen“ (BV11, 51). Alle Aktivist/innen bestätigen die Einschätzung, dass erfolgreiche Ultimaten die Bereitschaft zu kollektiven Aktionen inklusive Streiks steigert. Das gilt selbst dann, wenn das Ultimatum keinen oder nur einen unzureichenden Erfolg gebracht hat. Die betreffenden Teams seien dennoch zu „Streikteams“ geworden. „Die, die Ultimaten gemacht haben, die einmal so einen kollektiven Prozess durchgemacht haben, die streiken auch“ (SWV03, 40, 70).

Die Ultimaten gehen in der Regel mit einer Zunahme von Organisationsmacht einher. Ein ver.di-Sekretär berichtet von „,enormen Mitgliederzugewinnen“ (SV01, 85) in den beteiligten Teams. Eine Pflegekraft sagt: „Als wir das Teamversprechen oder den Notruf aufgesetzt hatten, waren wir zu 90 Prozent gewerkschaftlich organisiert, weil man das auf jeden Fall durch die Gewerkschaft begleiten lassen muss“ (CB10, 26). Die intensive Begleitung durch die Gewerkschaft ist offenbar in der Tat wichtig für den Erfolg von Ultimaten. Sie stellt „das nötige Know-how“ (SV01, 129) und rechtlichen Schutz zur Verfügung und fängt einen Großteil des Drucks durch den Arbeitgeber auf (SV01, 126-130).

Den Erzählungen zufolge befördert die erlebte „Kampf-Solidarität“ auch nach Ende der Ultimaten widerständiges Verhalten. Eine Krankenpflegerin berichtet, dass ihr Team sich seither ,,selbstbewusster gegenüber dem Arbeitgeber präsentiert und [...] gemeinsam [...] gegen vorherrschende Verhältnisse im Betrieb irgendwie vorgeht. [...] Bei uns war das tatsächlich so gewesen, dass da nicht mehr so irgendwie die üblichen Verdächtigen [...] Missstände angeprangert haben, sondern dass so nach und nach tatsächlich die Kollegen ein Selbstbewusstsein entwickelt haben und auf einmal mehrere Leute im Konflikt sich zu Wort gemeldet 
haben, was früher überhaupt nicht so gewesen ist“" (CB10, 26-28). Ein Personalrat berichtet von derselben Wirkung erfolgreicher Ultimaten: „Das stärkt auf jeden Fall das Team, also den Zusammenhalt. Die spüren auch die Macht, also das ist eindeutig von den Aussagen der Leute, dass sie merken, [...] dass es eine Auswirkung hat, was sie machen“ (SWV04, 24).

Auch auf das Verhalten der Klinikleitung in den alltäglichen Konflikten haben die Aktionen Auswirkungen. Viele berichten davon, dass sich die widerständigen Teams „Respekt verschafft" (SWV04, 10) haben und von den Führungskräften seither ernst genommen werden (SB02, 43; SV01, 64). „Das wirkt alles erst mal: ,Wir sind stark!““ (SV01, 78). Ein Krankenpfleger betont, dass dieser Effekt in der Regel länger anhält: „Das hat ja meistens auch nachhaltige Wirkung, dass wenn man einmal sich Respekt verschafft hat, dann reicht manchmal nur eine Mail, dass dann wieder was passiert, ohne dass wir jetzt einen großen Aufstand machen müssen“ (SWB04, 28).

\section{Fazit}

Die vorliegende Untersuchung behandelt nur einen Aspekt der veränderten Arbeitsbeziehungen im Krankenhaus. Die im Beitrag erwähnte neue Streikstrategie, die die Schließung von Betten und Stationen bewirken kann, wäre ebenfalls eingehender auf ihre Voraussetzungen und Wirkungen hin zu untersuchen. Gleiches gilt für das Zusammenspiel der verschiedenen Ebenen - der betrieblichen, tariflichen und der politischen - die ver.di in der Auseinandersetzung um mehr Personal und Entlastung im Krankenhaus nutzt (ver.di, 2017d). Welche Machtressourcen werden dabei ins Feld geführt? Wie korrelieren die Aktivitäten auf den verschiedenen Ebenen miteinander? Inwiefern ist die Gewerkschaft in der Lage, Marktgrenzen (zurück) zu verschieben? Welche Bündnisse geht sie dabei ein und welche Solidaritätspotenziale kann sie mobilisieren? Wie verlaufen die innergewerkschaftlichen Diskussionen zur strategischen und taktischen Ausrichtung? Welche Rolle spielen andere Akteure wie Staat, Klinikbetreiber und Krankenkassen? All diese Fragen wären eingehendere Studien wert, die den Rahmen dieser Arbeit bei Weitem gesprengt hätten.

Dennoch hat sie interessante Erkenntnisse zutage befördert, auch in Bezug auf Solidaritätskonzeptionen. Die Kollegialität - die im Alltag eine disziplinierende Wirkung entfaltet - wird durch die Ultimaten in eine „Kampf-Solidarität“ verwandelt, die die Durchsetzungsfähigkeit in Arbeitskämpfen, aber auch die Bereitschaft zu widerständigem Alltagsverhalten stärkt. Ihren formalisierten Ausdruck findet diese Solidarität im „Teamversprechen“, das die Pflegekräfte zumeist sogar schriftlich fixieren und unterschreiben. Damit gehen sie eine Art Pakt ein, sich kollektiv anders zu verhalten als im Alltag: Bei Inkrafttreten des Ultimatums gilt es nicht mehr als solidarisch, kurzfristig einzuspringen, sondern im Gegenteil, sich an die kollektiv beschlossene Aktion zu halten. Ob diese Transformation aber tatsächlich von Dauer ist oder - was zu vermuten ist - sich im Alltag wieder zurück entwickelt, kann in der vorliegenden Studie nicht geklärt werden.

In den Termini des Machtressourcenansatzes können die Ultimaten als Nutzung der (gestiegenen) Produktionsmacht von Pflegekräften verstanden werden. Schon die Verweigerung freiwilliger Leistungen birgt das Potenzial, die Abläufe in Krankenhäusern empfindlich zu stören. Werden die Ultimaten in Zusammenhang und als Vorbereitung von Streiks 
verstanden, bei denen es zur vorübergehenden Schließung von Betten und ganzen Stationen kommt, beinhalten sie ein ökonomisches Druckpotenzial, das so früher nicht bestand.

Eine wichtige Rolle in den Auseinandersetzungen um mehr Personal und Entlastung im Krankenhaus spielt zudem die Diskursmacht: Potenziell entscheidender als die Störung der Abläufe und die Verursachung eines betriebswirtschaftlichen Schadens ist der Reputationsverlust für die betreffenden Kliniken, der mit öffentlichkeitswirksamen Aktionen der Beschäftigten einhergehen kann. Die im Wettbewerb stehenden Krankenhäuser sind sehr darauf bedacht, ihren Ruf in der Bevölkerung zu wahren. Das können Beschäftigte bei Ultimaten oder anderen Aktionsformen nutzen, wenngleich die übermäßige mediale Skandalisierung der Zustände in einem konkreten Haus auch als riskant eingeschätzt wird. Eindeutig ist der Befund, dass erfolgreiche Ultimaten zur Steigerung von Organisationsmacht führen - nicht nur quantitativ bezogen auf Mitgliedszahlen, sondern vor allem durch die steigende Mobilisierungsfähigkeit und Eigenaktivität der beteiligten Beschäftigten.

Offen ist, inwieweit die betrieblichen, tarifpolitischen und öffentlichen Auseinandersetzungen in den Krankenhäusern tatsächlich zu einer Rück-Verschiebung der Marktgrenzen führen können. Einerseits ist der Prozess der Etablierung marktwirtschaftlicher Mechanismen im Krankenhauswesen weit fortgeschritten und erscheint nur schwer umkehrbar zu sein. Andererseits haben die gewerkschaftlichen Aktivitäten bereits erstaunliche Auswirkungen auf den gesellschaftlichen und politischen Diskurs. Union und SPD sahen sich dazu veranlasst, in ihrem Koalitionsvertrag nicht nur zu versprechen, „die Arbeitsbedingungen und die Bezahlung in der Alten- und Krankenpflege sofort und spürbar [zu] verbessern“, sondern auch, die „Pflegepersonalkosten besser und unabhängig von Fallpauschalen“ zu vergüten (Koalitionsvertrag, 2018, S. 96-99). Würden die Aufwendungen für das Pflegepersonal dem Preiswettbewerb tatsächlich vollständig entzogen, wäre das in der Tat eine bemerkenswerte Verschiebung von Marktgrenzen, die der „Logik des Marktkapitalismus“ (Aglietta, 2000) entgegenlaufen würde.

\section{Literaturverzeichnis}

Aglietta, M. (2000). Ein neues Akkumulationsregime. Die Regulationstheorie auf dem Prüfstand. Hamburg: VSA.

Arbeitskreis Strategic Unionism (2013). Jenaer Machtressourcenansatz 2.0. In S. Schmalz \& K. Dörre (Hrsg.), Comeback der Gewerkschaften? Machtressourcen, innovative Praktiken, internationale Perspektiven (S. 345-375). Frankfurt/Main: Campus.

Artus, I., Birke, P., Kerber-Clasen, S. \& Menz, W. (Hrsg.). (2017). Sorge-Kämpfe. Auseinandersetzungen um Arbeit in sozialen Dienstleistungen. Hamburg: VSA.

Baum, R. C. (1975). The System of Solidarities. Indian Journal of Sociology, 16/1975, 306-353.

Bayertz, K. (1998). Begriff und Problem der Solidarität. In K. Bayertz (Hrsg.), Solidarität - Begriff und Problem (S. 11-53). Frankfurt/Main: Suhrkamp.

Becker, K. (2014). Von Florence Nightingale zu Adam Smith? zfwu, 15 (1), 33-52. doi: 10.1688/zfwu-2014-01-becker

Becker, K. (2016). Loyale Beschäftigte - ein Auslaufmodell? Pflege \& Gesellschaft, 2/2016, 145161. doi: $10.3262 / \mathrm{P} \& G 1602145$

Behrens, M. \& Dribbusch, H. (2014). Arbeitgebermaßnahmen gegen Betriebsräte: Angriffe auf die betriebliche Mitbestimmung. WSI-Mitteilungen, 2/2014, 140-148.

doi: 10.5771/0342-300X-2014-2-140 
BibliomedPflege (2018). Unbefristete Streiks an Uniklinika Essen und Düsseldorf. Abgerufen am 27. August 2018 von

https://www.bibliomed-pflege.de/alle-news/detailansicht/36031-unbefristete-streiks-anuniklinika-essen-und-duesseldorf/

Billmann, L. \& Held, J. (2013). Einführung. Solidarität, kollektives Handeln und Widerstand. In L. Billmann \& J. Held (Hrsg.). Solidarität in der Krise: Gesellschaftliche, soziale und individuelle Voraussetzungen solidarischer Praxis (S. 13-29). Tübingen: Springer VS.

Bingener, R. (2012). Eine Bestätigung für die Kirchen. Frankfurter Allgemeine Zeitung vom 20. November 2012. Abgerufen am 8. August 2018 von http://www.faz.net/aktuell/politik/inland/urteil-zum-streikrecht-eine-bestaetigung-fuer-diekirchen-11966698.html

Bischoff, C. (1992). Frauen in der Krankenpflege. Frankfurt am Main/New York: Campus.

Bode, I. (2010). Die Malaise der Krankenhäuser. Leviathan, 38, 189-211. doi: 10.1007/s11578-010-0082-x

Braun, M. (2015). Ludwigsburg: Streit am Krankenhaus eskaliert. Stuttgarter Zeitung vom 9. Juni 2015. Abgerufen am 2. Mai 2018 von https://www.stuttgarter-zeitung.de/inhalt.ludwigsburg-streit-am-krankenhaus-eskaliert.3a67482345dc-455e-acc2-e1309f9b5162.html

Bräutigam, C., Evans, M., Hilbert, J. \& Öz, F. (2014). Arbeitsreport Krankenhaus. Eine Online-Befragung von Beschäftigten deutscher Krankenhäuser. Arbeitspapier 306. Düsseldorf: Hans-BöcklerStiftung.

Brinkmann, U. (2011). Die unsichtbare Faust des Marktes: betriebliche Kontrolle und Koordination im Finanzmarktkapitalismus. Berlin: edition Sigma.

Brinkmann, U. \& Nachtwey, O. (2010). Krise und strategische Neuorientierung der Gewerkschaften. Aus Politik und Zeitgeschichte, 13-14/2010, 21-29.

Brinkmann, U., Choi, H.-L., Detje, R., Dörre, K., Holst, H., Karakayali, S. \& Schmalstieg, C. (2008). Strategic Unionism: Aus der Krise zur Erneuerung? Umrisse eines Forschungsprogramms. Wiesbaden: VS Verlag für Sozialwissenschaften.

Brookes, M. (2018). Power Resources in Theory and Practice: Where to go from here. Global Labour Journal 9 (2), 2018, 254-257.

Cropley, A. J. (2002). Qualitative Forschungsmethoden - eine praxisnahe Einführung. Eschborn: Klotz.

Deutscher Berufsverband für Pflegeberufe (DBfK). (2016). Mein Recht auf Frei. Abgerufen am 05. Dezember 2017 von https://www.dbfk.de/media/docs/download/Allgemein/Mein-Recht-auf-Frei_Sammelband-2016.pdf

Dörre, K. (2011). Funktionswandel der Gewerkschaften. Von der intermediären zur fraktalen Organisation. In T. Haipeter \& K. Dörre (Hrsg.), Gewerkschaftliche Modernisierung (S. 267-301). Wiesbaden: VS Verlag für Sozialwissenschaften.

Durkheim, E. (1992 [1893]). Über soziale Arbeitsteilung. Studie über die Organisation höherer Gesellschaften. Frankfurt/Main: Suhrkamp.

Ebbinghaus, B. \& Visser, J. (1999). When Institutions Matter. Union Growth and Decline in Western Europe, 1950-1995. European Sociological Review, 15 (2), 135-158. doi: 10.1093/oxfordjournals.esr.a018257

Folbre, N. (2001). The Invisible Heart: Economics and Family Values. New York: New Press.

Hartfiel, G. \& Hillmann, K.-H. (1972). Wörterbuch der Soziologie. Stuttgart: Kröner.

Haug, W.F. (2009). Gewerkschaften im High-Tech-Kapitalismus vor der Hegemoniefrage. Das Argument, 51 (6), 879-893.

Hechter, M. (1988). Principles of Group Solidarity. Berkeley: University of California Press. 
Hipp, L. \& Kelle, N. (2015). Nur Luft und Liebe? Die Entlohnung sozialer Dienstleistungsarbeit im Länder- und Berufsvergleich. Friedrich-Ebert-Stiftung. Forum Politik und Gesellschaft. Berlin: FES.

Hirsch-Kreinsen, H. (2010). Entgrenzung von Unternehmen und Arbeit. In C. Deutschmann \& J. Beckert (Hrsg.), Wirtschaftssoziologie (S. 447-465). Wiesbaden: VS Verlag.

Honneth, A. (1992). Kampf um Anerkennung. Frankfurt am Main: Suhrkamp.

Hürtgen, S. (2013). Mensch sein auf der Arbeit? Kollegialität als Balance von allgemein-menschlichen und leistungsbezogenen Aspekten von Arbeit. In L. Billmann \& J. Held (Hrsg.). Solidarität in der Krise: Gesellschaftliche, soziale und individuelle Voraussetzungen solidarischer Praxis (S. 237-262). Tübingen: Springer VS.

Kirch, D. (2018). Verdi droht mit langem Streik an Uniklinik Homburg. Saarbrücker Zeitung vom 8. Juni 2018. Abgerufen am 27. August 2018 von

https://www.saarbruecker-zeitung.de/saarland/saarland/verdi-droht-mit-langem-streik-anuniklinik-homburg_aid-23316423

Koalitionsvertrag (2018). Ein neuer Aufbruch für Europa. Eine neue Dynamik für Deutschland. Ein neuer Zusammenhalt für unser Land. Berlin: CDU, CSU, SPD.

Kröll, T. (2013). Arbeitnehmersolidarität im neoliberalen Strukturwandel. In L. Billmann \& J. Held (Hrsg.). Solidarität in der Krise: Gesellschaftliche, soziale und individuelle Voraussetzungen solidarischer Praxis (S. 78-97). Tübingen: Springer VS.

Lamnek, S. (2005). Qualitative Sozialforschung. Lehrbuch. Weinheim/Basel: Beltz.

Marrs, K. (2007). Ökonomisierung gelungen, Pflegekräfte wohlauf? WSI-Mitteilungen, 9/2007, 502507. doi: 10.5771/0342-300x-2007-9-502

Marx, K. (1972 [1867]). Instruktionen für die Delegierten des Provisorischen Zentralrats zu den einzelnen Fragen. In Marx Engels Werke (MEW) (Bd. 16, S. 196), Berlin: Dietz.

Mayring, P. (2002). Einführung in die qualitative Sozialforschung. Eine Anleitung zu qualitativem Denken. Weinheim und Basel: Beltz.

Mayring, P. (1994). Qualitative Inhaltsanalyse. In A. Boehm, A. Mengel \& T. Muhr (Hrsg.), Texte verstehen (S. 159-175). Konstanz: Universitätsverlag Konstanz.

Müller-Jentsch, W. (1997). Soziologie der industriellen Beziehungen - Eine Einführung. Frankfurt/Main: Campus.

Peters, K. \& Sauer, D. (2005) Indirekte Steuerung - eine neue Herrschaftsform. Zur revolutionären Qualität des gegenwärtigen Umbruchprozesses. In H. Wagner (Hrsg.), „Rentier’ ich mich noch?“ Neue Steuerungskonzepte im Betrieb (S. 23-58). Hamburg: VSA.

Rieder, K. (1999). Zwischen Lohnarbeit und Liebesdienst: Belastungen in der Krankenpflege. Weinheim/München: Juventa.

Rosenbrock, R. \& Gerlinger, T. (2014). Gesundheitspolitik. Eine systematische Einführung. Bern: Huber.

Roth, I. (2011). Die Arbeitsbedingungen in Krankenhäusern aus Sicht der Beschäftigten. Ein Branchenbericht auf Basis des DGB-Index Gute Arbeit. Berlin: ver.di Bereich Innovation und Gute Arbeit.

Scherr, A. (2013). Solidarität im Postmodernen Kapitalismus. In L. Billmann \& J. Held (Hrsg.), Solidarität in der Krise: Gesellschaftliche, soziale und individuelle Voraussetzungen solidarischer Praxis (S. 263-270). Tübingen: Springer VS.

Schmalz, S., Ludwig, C. \& Webster, E. (2018). The Power Resources Approach: Developments and Challenges. Global Labour Journal 9(2), 2018, 113-134. doi: 10.15173/glj.v9i2.3569

Senghaas-Knobloch, E. (2008). Zeit für fürsorgliche Praxis. Pflegeethos und Erfahrungen von Frauen und Männern in Pflegeberufen. In E. Senghaas-Knobloch \& C. Kumbruck (Hrsg.), Vom Liebesdienst zur liebevollen Pflege (S. 77-94). Rehburg-Loccum: Evangelische Akademie Loccum.

Siemens, S. \& Frenzel, M. (2016). Das unternehmerische Wir. Formen der Indirekten Steuerung in Unternehmen. Hamburg: VSA. 
Silver, B. J. (2005). Forces of Labor. Arbeiterbewegungen und Globalisierung seit 1870. Berlin/Hamburg: Assoziation A.

Simon, K. (2018). Was der Streik an der Uniklinik für die Patienten bedeutet. Artikel vom 3. August 2018 bei WAZ-Online. Abgerufen am 8. August 2018 von https://www.waz.de/staedte/essen/was-der-streik-an-der-uniklinik-fuer-die-patienten-bedeutetid215006567.html

Simon, M. \& Mehmecke, S. (2017). Nurse-to-Patient Ratios: Ein internationaler Überblick über staatliche Vorgaben zu einer Mindestbesetzung im Pflegedienst der Krankenhäuser. Working Paper 27. Düsseldorf: Hans-Böckler-Stiftung.

Simon, M. (2016). Die ökonomischen und strukturellen Veränderungen des Krankenhausbereichs seit den 1970er Jahren. In I. Bode \& W. Vogd (Hrsg.), Mutationen des Krankenhauses (S. 29-45). Wiesbaden: Springer.

Simon, M. (2013). Das DRG-System. Grundsätzliche Konstruktionsfehler. Deutsches Ärzteblatt, 39, $1782-1786$.

SpiegelOnline (2018). Fachkräftemangel: Deutschland fehlen mehr als 25.000 Pfleger. Artikel vom 25. April 2018. Abgerufen am 8. August 2018 von http://www.spiegel.de/wirtschaft/soziales/pflege-deutschland-fehlen-mehr-als-25-000fachkraefte-a-1204648.html

Statistisches Bundesamt (2018). Grunddaten der Krankenhäuser 2016, Fachserie 12 Reihe 6.1.1.

Streeck, W. (1979). Gewerkschaften als Mitgliederverbände. Probleme gewerkschaftlicher Mitgliederrekrutierung. In J. Bergmann (Hrsg.), Beiträge zur Soziologie der Gewerkschaften (S. 72110). Frankfurt/Main: Suhrkamp.

Supe, J. (25. Januar 2018). Die Drohung, normal zu arbeiten. junge Welt, 5.

Tenfeld, K. (1998). Arbeiterschaft, Solidarität du Arbeiterbewegung. In K. Bayertz (Hrsg.), Solidarität - Begriff und Problem (S. 195-201). Frankfurt/Main: Suhrkamp.

Thome, H. (1998). Soziologie und Solidarität: Theoretische Perspektiven für die empirische Forschung. In K. Bayertz (Hrsg.), Solidarität - Begriff und Problem (S. 217-262). Frankfurt/Main: Suhrkamp.

Tranow, U. (2012). Das Konzept der Solidarität. Düsseldorf: Springer VS.

Urban, H.-J. (2010). Wohlfahrtsstaat und Gewerkschaftsmacht im Finanzmarkt-Kapitalismus: Der deutsche Fall. WSI-Mitteilungen, 9/2010, 443-450. doi: 10.5771/0342-300X-2010-9-443

Van Parijs, Ph. (1996). Refondé la Solidarité. Paris: Editions du Cerf.

Van Parijs, Ph. (1995). Sauver la Solidarité. Paris: Editions du Cerf.

ver.di (2018a). Celenus taucht ab. Abgerufen am 8. August 2018 von https://gesundheit-soziales.verdi.de/mein-arbeitsplatz/reha-einrichtungen/++co++c7d71a107635-11e8-972a-525400f67940

ver.di (2018b). Entlastung oder Streik. Abgerufen am 13. August 2018 von https://gesundheit-soziales.verdi.de/themen/entlastung/++co++a78f50f6-755e-11e8-b3f9$525400 \mathrm{ff} 2 \mathrm{~b} 0 \mathrm{e}$

ver.di (2018c). Mehr von uns ist besser für alle. Abgerufen am 13. August 2018 von https://gesundheit-soziales.verdi.de/themen/entlastung/++co++3991a0d2-3be8-11e7-bd01$525400423 \mathrm{e} 78$

ver.di (2017a). Mach' mal Pause. Abgerufen am 02. Mai 2018 von http://gesundheit-soziales.verdi.de/themen/entlastung/++co++dc9740ce-e248-11e6-bc13$525400423 \mathrm{e} 78$

ver.di (2017b). Pflegenotstand: Was auf der Strecke bleibt. Abgerufen am 02. Mai 2018 von http://gesundheit-soziales.verdi.de/themen/mehr-personal/++co++7a9029c2-371b-11e7-91e4$52540066 \mathrm{e} 5 \mathrm{a} 9$ 
ver.di (2017c). Händedesinfektion? Klar, nur wann? Abgerufen am 02. Mai 2018 von

http://gesundheit-soziales.verdi.de/themen/entlastung/++co++b4a07b28-6e05-11e7-8b0a$525400 \mathrm{ff} 2 \mathrm{~b} 0 \mathrm{e}$

ver.di (2017d). Mehr von uns ist besser für alle. Abgerufen am 11. Mai 2018 von

http://gesundheit-soziales.verdi.de/themen/entlastung/++co++3991a0d2-3be8-11e7-bd01$525400423 \mathrm{e} 78$

ver.di (2017e). Union Busting: „Krachend gescheitert“. Abgerufen am 8. August 2018 von

https://gesundheit-soziales.verdi.de/themen/entlastung/++co++0ea8eeb4-dfff-11e7-8a4a$525400423 \mathrm{e} 78$

ver.di (2016). Aktion Überstundenberg. Abgerufen am 05. Dezember 2017 von

https://gesundheit-soziales.verdi.de/themen/mehr-personal/++co++cf611028-c16f-11e6-bf70$525400 \mathrm{ed} 87 \mathrm{ba}$

ver.di (2015). Gegendruck. Hilfe zur Selbsthilfe bei Überlastung und Personalnot. Berlin: ver.diFachbereich Gesundheit, Soziale Dienste, Wohlfahrt und Kirchen.

ver.di b+b (2017). Überlastungsanzeige. Abgerufen am 02. Mai 2018 von https://www.verdi-bub.de/service/praxistipps/archiv/ueberlastungsanzeige

ver.di Berlin-Brandenburg. (o. J.). Mehr von uns ist besser für alle. Der Kampf um Entlastung und Gesundheitsschutz an der Berliner Charité. Berlin: ver.di-Landesbezirk Berlin-Brandenburg.

ver.di Region Saar-Trier. (o. J.). Die Ultimaten in der ver.di-Region Saar-Trier. Saarbrücken: ver.diFachbereich 03 der ver.di-Region Saar-Trier.

Visser, J. (1995). Trade unions from a comparative perspective. In J. Van Ruysseveldt, R. Huiskamp, \& J. Hoof (Hrsg.), Comparative industrial and employment relations (S. 37-67). London, Sage.

Vogd, W. (2006). Die Organisation Krankenhaus im Wandel. Eine dokumentarische Evaluation aus Sicht der ärztlichen Akteure. Bern: Huber.

Voges, W. (2002). Pflege alter Menschen als Beruf: Soziologie eines Tätigkeitsfeldes. Wiesbaden: Westdeutscher Verlag.

Wildt, A. (1998). Solidarität - Begriffsgeschichte und Definition heute. In K. Bayertz (Hrsg.), Solidarität - Begriff und Problem (S. 202-216). Frankfurt/Main: Suhrkamp.

Windisch, W. (2017). »Wir haben es selbst in der Hand, noch stärker zu werden!« Die Bewegung für einen Tarifvertrag Entlastung in den saarländischen Krankenhäusern. In I. Artus, P. Birke, S. Kerber-Clasen \& W. Menz (Hrsg.), Sorge-Kämpfe, Auseinandersetzungen um Arbeit in sozialen Dienstleistungen (S. 130-153). Hamburg: VSA.

Wright, E. O. (2000). Working Class Power, Capitalist Class Interests, and Class Compromise. American Journal of Sociology, 105 (4), 957-1002. doi: 10.1086/210397 


\title{
Horan Lee \& Ronald Staples*
}

\section{Digitale Solidarität unter Arbeitnehmer*innen**}

\section{Zusammenfassung}

In der modernen Arbeitswelt individualisieren sich Arbeitsverhältnisse zusehends. Die Orientierung an einem Kollektiv wird abgelöst von einem radikalen Durchsetzen von Partikularinteressen. Solidarität als Basiskategorie von Arbeitnehmeridentität gerät damit unter Druck. Auch Gewerkschaften laufen Gefahr, nur noch partikulare Interessen zu vertreten, wenn der Einzelne zum Maßstab des Handelns wird. Arbeitnehmer*innen in digitalen Arbeitsfeldern gelten als die Speerspitzen einer individualisierten Lebens- und Arbeitswelt. Der Artikel erarbeitet zum einen ein mehrdimensionales Konzept von organischer Solidarität und zeigt dann, wie auch in digital mediatisierten Arbeitsumgebungen solidarische Beziehungen wirksam sind. Angewandt auf drei drängende Problemfelder von Arbeit wird die Frage gestellt, inwiefern digitale Solidarität eine Chance zur Bearbeitung dieser Probleme darstellen kann.

Schlagwörter: Solidarität, digitale Transformation, digital Commons, digitale Allmenden, Arbeit, Durkheim

\section{Digital Solidarity among workers}

\begin{abstract}
In the modern working world, working relationships are becoming increasingly individualized. The orientation towards a collective is replaced by a radical assertion of particular interests. Solidarity as the basic concept of an employee's identity is thus coming under pressure. Trade unions also run the risk of representing only particular interests if the individual becomes the yardstick for action. Employees in digital fields of work are considered the spearheads of an individualized world of life and work. The article develops a multidimensional concept of organic solidarity and then shows how solidarity relations are also effective in digitally mediatized working environments. Applied to three

* Dr. Horan Lee, Friedrich-Alexander-Universität Erlangen-Nürnberg, Institut für Soziologie, Kochstraße 4, 91054 Erlangen. E-Mail: horan.lee@fau.de

Dr. Ronald Staples, Friedrich-Alexander-Universität Erlangen-Nürnberg, Institut für Soziologie, Kochstraße 4, 91054 Erlangen. E-mail: ronald.staples@ fau.de

** Artikel eingegangen: 15.05.2018, revidierte Fassung akzeptiert nach doppelt-blindem Begutachtungsverfahren: 10.09 .2018
\end{abstract}


pressing problem areas of work, the question is asked to what extent digital solidarity can represent an opportunity to solve these problems.

Keywords: Solidarity, digital transformation, commons, labour, Durkheim (JEL: J5, O33, P13)

\section{Einleitung}

Die Digitalisierung der Arbeitswelt verändert die industriellen Beziehungen nicht nur in ihrer institutionellen Form, sondern auch in ihrem substanziellen Kern, denn Arbeitsverhältnisse und deren arbeitspolitische Aushandlung sind in digitalen Kontexten noch stärker individualisierbar bzw. schwerer einer kollektiven Regulierungspraxis zu unterwerfen (vgl. Boes, Kämpf, Langes \& Lühr, 2015; Pfeiffer, 2017). ${ }^{1}$ Damit wird auch die Frage nach der Solidarität unter Arbeitnehmer*innen (neu) aufgeworfen. Dabei gehen wir nicht davon aus, dass Solidarität als Quelle von Verhandlungsmacht für die Arbeitnehmerseite obsolet wird, sich jedoch Form und Charakter von Solidarität in digitalen Arbeitswelten in anderer Weise dar- und herstellen, als im Common Sense gewerkschaftlicher 'Arbeitersolidarität'. Den besonderen Konstitutionsbedingungen und Erscheinungsformen einer möglichen 'digitalen Solidarität' unter Arbeitnehmer*innen wollen wir in diesem Beitrag nachgehen und aufzeigen, inwiefern die Akteure der industriellen Beziehungen mittels dieser Re-Konzeptualisierung von Solidarität gegenwärtigen Herausforderungen der Interessenvertretung von Arbeitnehmer*innen produktiv begegnen können.

Der vorliegende Beitrag nähert sich einer begrifflichen Bestimmung digitaler Solidarität von zwei Seiten. Ausgehend von ausgewählten soziologischen Arbeiten zur Solidarität werden einerseits theoretische Grundzüge herausgearbeitet, die wir als relevant für die gegenwärtige, zunehmend digitalisierte Arbeitswelt interpretieren. Andererseits greifen wir die von Felix Stalder (2013) anhand empirischer Beispiele skizzierten vier Formen digitaler Solidarität auf (Commons, Assemblies, Swarms und weak Networks), um sie mit unseren theoretisch hergeleiteten Dimensionen digitaler Solidarität zu konfrontieren. Diese Formen digitaler Solidarität übertragen wir auf drei konkrete Problemkontexte in den industriellen Beziehungen, um Solidaritätspotenziale in einer digitalisierten Arbeitswelt sichtbar zu machen. Eine eigene empirische Untersuchung zur Praxis digitaler Solidarität unter Arbeitnehmer*innen steht noch aus, ist jedoch in den Fragestellungen eines derzeit laufenden Forschungsprojekts mit angelegt. ${ }^{2}$

Den Hintergrund der hier behandelten Thematik bildet die verlorengegangene Selbstverständlichkeit, nach der größere Kollektive mit einer gemeinsamen Identität ausgestattet sind, an die sich unmittelbar ein solidarischer Zusammenhalt knüpft. Sich affirmativ auf andere zu

1 Die Digitalisierung sehen wir keineswegs deterministisch als technikinduzierten Imperativ, der Veränderungen schlicht vorgibt. Aber im Zuge einer unbestreitbaren technischen Mediatisierung von Kommunikation sowie als inzwischen omnipräsenter Diskurs hat die Digitalisierung eine Wirkmächtigkeit erlangt, der sich die Akteure der industriellen Beziehungen nicht mehr entziehen können.

2 Das Projekt „Partizipative Mitbestimmung in digitalisierter Arbeitswelt“ im Forschungsverbund „Digitalisierung, Mitbestimmung, Gute Arbeit“ wird von der Hans-Böckler-Stiftung gefördert und ist im Oktober 2017 gestartet. 
beziehen und den stabilen Bezug auf ein Kollektiv als Ressource zur Konstruktion von Identität zu sehen, steht im Zeitalter der „Singularitäten“ (Reckwitz, 2017) gesellschaftlich also insgesamt auf dem Prüfstand. Die Zugehörigkeit zu einem übergeordneten Bedeutungszusammenhang ist legitimierungsbedürftig geworden, da a) der Einzelne im Mittelpunkt steht oder stehen will und b) es doch eine Vielzahl von wählbaren, einander überlagernden sozialen Kreisen gibt, wie etwa Kirchen, Sportvereine, Parteien, NGOs, Firmen, youtube-Kanäle etc., woraus dann Individualität gewonnen wird (Simmel, 1992, S. 489).

Die Unsicherheiten rund um den Zwang zur Wahl haben schon seit längerem die Arbeitswelt und damit auch die Arbeitnehmerinteressenvertretungen erreicht (vgl. Beck \& Lau, 2005, S. 113). Spätestens seit im Zuge der Subjektivierung von Arbeit die gesamte Person in den Fokus der Aufmerksamkeit von Arbeitgeber*innen gerückt ist und nicht nur die mit einer Stelle eng verknüpften beruflichen Fertigkeiten, müssen Gewerkschaften und Betriebsräte ihren Wert für die Beschäftigten unter Beweis stellen (Voß \& Pongratz, 1998). Wenn Unternehmen Arbeitsumgebungen schaffen, die die scharfe Trennung von Leben und Arbeit verwischen und die Beschäftigten qua Partizipation in Entscheidungsprozesse miteinbinden (Hippel, 2005), dann laufen Interessensvertretungen Gefahr, als primäre Repräsentationsinstanz von Erwerbsarbeit an Bedeutung einzubüßen: Eine Kollektividentität als homogenes Arbeiter-Wir hat als solidaritätsstiftendes Klassenkampfnarrativ zunehmend ausgedient.

Allerdings sind neben der nach wie vor bestehenden Bereitschaft zu gewerkschaftlicher Organisation offensichtlich auch in neueren, digitalisierten Arbeits- und Interaktionsformen Kollektivierungstendenzen $\mathrm{zu}$ erkennen, schließlich finden freiwillige Kooperationszusammenschlüsse z.B. im Rahmen der Open-Source-Bewegung großen Anklang. In der Zivilgesellschaft und insbesondere im bzw. über das Internet sind in den letzten 15 Jahren kollaborative Netzwerke entstanden, die stark auf Einbindung und Engagement qua Partizipation ihrer ,Mitglieder' setzen, ohne jedoch zu versuchen, deren Individualität den Zielen der Organisation direktiv unterzuordnen. Die Darstellung unverwechselbarer Individualität und Partizipation in Gemeinschaften als Ko-laboration und Co-creation (Brown, 2009; Gauntlett, 2007; Reckwitz, 2012) scheinen mächtige Leitmotive eines modernen Arbeitsnarrativs geworden zu sein.

Droht der institutionalisierten Interessenvertretung, die Deutungsmacht zur Kollektivierung von Arbeitnehmeridentität zu verlieren? Da Solidarität auch weiterhin als zentrale Gelenkstelle in der Arbeitswelt und ihrer arbeitspolitischen Aushandlung zu verstehen ist, sollte die Frage u.E. anders gestellt werden: Können die in digitalen Kontexten aufkommenden Formen von Solidarität im Rahmen institutionalisierter Interessenvertretung (besser) genutzt werden oder sogar grundsätzlich in die Prinzipien der interessenpolitischen Organisation von Arbeitskraft einfließen? Die Attraktivität, die sich mit digitaler Co-Creation verknüpft und an der es der institutionalisierten Interessensvertretung speziell für Wissens- und Kreativarbeiter*innen offenbar mangelt, erscheint in einem anderen Licht, wenn man Solidarität als zentralen Integrationsmodus digitaler Communities wie Open-Source-Projekte, creative Commons oder andere Kooperationsnetzwerke im Internet näher betrachtet.

Die Grundzüge einer solchen digitalen Solidarität leiten wir im Folgenden aus den theoretischen Reflexionen zur organischen Solidarität (nach Durkheim), zu Anerkennung (nach Honneth) und emotionalen Aspekten von Solidarität (nach Hondrich \& Koch- 
Arzberger, 1992) her, um so zu einem mehrdimensionalen Konzept von digitaler Solidarität zu gelangen (Abschnitt 2). Anschließend stellen wir die von Felix Stalder identifizierten typischen Erscheinungsformen digitaler Solidarität vor und setzen sie in Bezug zu dem zuvor entwickelten Theoriegerüst (Abschnitt 3). In einem dritten Schritt beziehen wir dann das neu gewonnene Verständnis von digitaler Solidarität auf drei exemplarische Herausforderungen in den industriellen Beziehungen der modernen Arbeitswelt und diskutieren Bearbeitungsmöglichkeiten durch die Akteure der institutionalisierten Interessenvertretung (Abschnitt 4). In einem abschließenden Fazit leiten wir einige mögliche Konsequenzen für die Zukunft der Solidarität unter Arbeitnehmer*innen ab, die sich aus den besonderen Eigenarten digitaler Solidarität ziehen lassen und bieten Anschlussmöglichkeiten für weitere Forschungsarbeit an (Abschnitt 5).

\section{Solidarität heute - eine theoriegeleitete Konzeptualisierung}

Was ,Solidarität' ist, wird meist präreflexiv verstanden und führt in verschiedenen Feldern solidarischer Praxis kaum zu größeren Missverständnissen, was auf ein breites Fundament intersubjektiv geteilter Bedeutungsgehalte der Semantik verweist. Im theoretischen Zugriff wird jedoch evident, dass die diversen wissenschaftlich systematisierten Definitionsversuche von Solidarität weder Allgemeingültigkeit beanspruchen noch Eindeutigkeit hervorbringen können. Solidaritätskonzepte unterscheiden sich inhaltlich und in der Schwerpunktsetzung je nach wissenschaftlich-disziplinärer Herkunft, in historischer Perspektive und hinsichtlich des konkreten gesellschaftlichen Phänomenbereichs. Insofern soll die folgende Darstellung eines konzeptuellen Rahmens für die ,Digitale Solidarität unter Arbeitnehmer*innen' die bewusst eklektische Theoriebasis für diesen Solidaritätstypus erläutern, und zwar vor dem Hintergrund einer spezifischen historisch-institutionellen Verortung in den industriellen Beziehungen.

Durkheims bekannte Erklärung dafür, warum der Fortbestand sozialer Ordnung im Zuge gesellschaftlicher Modernisierung trotz der - aufgrund der sozialen bzw. nach beruflicher Spezialisierung differenzierten Arbeitsteilung - gestiegenen Heterogenität möglich ist, liegt in der Verschiebung von mechanischer zu organischer Solidarität als dominantem gesellschaftlichen Modus der Sozialintegration. Nicht mehr ausschließlich die kollektividentitätsstiftende Gleichheit oder Ähnlichkeit, sondern zunehmend Differenzen (qua Spezialisierung) zwischen Gesellschaftsmitgliedern begründen solidarische Beziehungen untereinander (Durkheim, 1977). Diese differenztheoretische Sichtweise auf Solidarität sehen wir als elementaren Baustein an zur Neubestimmung von Solidarität unter digitalen Vorzeichen, allerdings mit einigen Relativierungen und Erweiterungen der Durkheimschen Solidaritätsthesen. ${ }^{3}$

3 Die Umstellung des Solidaritätsmodus hat auch auf Makroebene sichtbare Konsequenzen, bspw. in den Beziehungen europäischer Nationalstaaten und deren Ausgestaltung von Sozialsystemen (Münch \& Büttner, 2006). 
Die Gleichheitsbedingung in der organischen Solidaritätsform nach Dürkheim verschwindet keineswegs vollständig, vielmehr bildet das „Verhältnis von Differenz und Gleichheit - in welcher Proportion auch immer - die Grundlage von ,organischer' Solidarität. Die Differenz wird wahrgenommen, ja ausdrücklich thematisiert, und zugleich die Gleichheit - meist auf einer abstrakten Ebene, gar auf der abstraktesten (auch ein menschliches Wesen) - gesehen und die Schlussfolgerung gezogen: Diese abstrakte Gleichheit ist ein hinreichender Grund für Solidarität“" (Zoll, 2000, S. 166). Der Unterschied zwischen mechanischer und organischer Solidarität ist demnach kein diskreter und nur bedingt einer von gesellschaftlichen Formationen. Organische Solidarität beruht nicht ausschließlich auf Differenz, zum Teil speist sie sich lediglich aus einer allgemeineren bzw. ,schwächeren“ Definition von Gleichheit und akzeptiert somit ein höheres Maß an Unterschiedlichkeit hinsichtlich weiterer Merkmale. Das ,soziale Band' der organischen Solidarität ist flexibler und daher strapazierfähiger im ,aushalten“ von Unterschieden, geknüpft wird es dennoch auf der Basis von mindestens zwei intersubjektiv geteilten Gemeinsamkeiten. Während die erste in einer generalisierten Gleichheitsunterstellung wurzelt (zur bisher dominanten Bildung sozialer Identität ${ }^{4}$ durch Beruf und/oder Arbeitsplatz treten heute Freizeitinteressen, sexuelle Orientierung, Geschlechts- oder Generationenzugehörigkeit immer stärker in Konkurrenz), besteht die zweite Gemeinsamkeit in der Ausrichtung auf übergeordnete Zielhorizonte, die durch arbeitsteilig spezialisierte, sich jeweils ergänzende Einzelbeiträge kollaborativ verfolgt werden. Insofern wird organische Solidarität nicht durch beliebige Differenzen, sondern durch komplementäre Unterschiedlichkeit auf der Grundlage steigender Interdependenz gestiftet. Eine dichotome Gegenüberstellung von mechanischer und organischer Solidarität mit Rückgriff auf eine entweder in Großgruppen oder auf Grundlage individualisierter Arbeitnehmeridentität(en) entstehende Kollektivität reicht für eine konzise begriffliche Bestimmung digitaler Solidarität nicht aus. Mechanische Solidarität ist ja weiterhin eine soziale Tatsache, organische Solidarität spielt allerdings die deutlich größere Rolle für Sozialintegration in modernen Gesellschaften. In Felix Stalders Zeitdiagnose zur Entwicklung einer „Kultur der Digitalität“ (2016) wird digitale Solidarität als Gleichzeitigkeit von Differenz und Gemeinsamkeit konzeptualisiert, wie in Abschnitt 2 noch diskutiert wird (vgl. Stalder, 2016, S. 141).

Im Kontext der industriellen Beziehungen sind die Durkheimschen Solidaritätsthesen arbeitnehmerseitig zwar anknüpfbar, müssen jedoch machttheoretisch erweitert werden. Denn während organische Solidarität prinzipiell auch ohne Gegnerbezug denkbar ist, bildet die historische Kollektiverfahrung der Arbeiter- und Gewerkschaftsbewegung gegen die Kapitalseite Solidarität üben zu müssen, ihren konstitutiven Ausgangspunkt. Hieraus speist sich bis heute der normative Kern der Solidaritätssemantik im Klassenkampfdispositiv zur Institutionalisierung von Interessenpolitik (Müller-Jentsch, 1997). Während sich jedoch zur gewerkschaftlichen Organisierung von Arbeit bis heute in erster Linie das Modell der mechanischen Klassensolidarität eingespielt hat und dabei insbesondere in Deutschland der männliche Facharbeiter ohne Migrationshintergrund zum prototypischen Vertreter einer

4 Ebenso wie ,Solidarität “ verstehen wir den Begriff ,Identität‘ als interpretationsoffene und kontextualisierungsbedürftige Kategorie. Besonders im Rekurs auf (behauptete) Gruppenidentitäten werden die normativen Semantiken/Praktiken erkennbar, mit denen Identitätspolitik durch Thematisierung, Zuschreibung bzw. Absprechung bestimmter Gleichheitsmerkmale betrieben wird. 
kollektiven Arbeiteridentität avanciert ist, gerät dieser Integrationsmodus im Zuge der zunehmenden Auflösung traditioneller Arbeitermilieus und der Heterogenisierung von Erwerbstätigkeit unter Druck (vgl. hierzu auch Kap. 3.1). Im Sinne Hymans müssten Gewerkschaften künftig Identifikation eher qua „,common interests“ und „mutuality despite difference“ herstellen (Hyman, 2002, S. 1-2) - also auf organische Solidarität setzen - wenn sie in Zukunft noch eine relevante Rolle als mitgliederstarke Kollektivmacht spielen wollen. Der wertschätzenden Anerkennung (statt lediglich Toleranz) von Unterschiedlichkeit vor dem Hintergrund wechselseitiger Abhängigkeit kommt in diesem Zusammenhang eine besondere Bedeutung zu. Anerkennungsverhältnisse verweisen dabei auf eine Analyseebene von solidarischen Beziehungen, die mit dem strukturalistischen Ansatz Durkheims kaum erfasst werden kann, in der Theorie der Anerkennung von Axel Honneth jedoch explizit thematisiert wird. In dieser werden Anerkennungsverhältnisse intersubjektiv gedacht und von ihrem dyadischen Konstitutionspunkt aus auf aggregierte Akteure transponiert (wie bspw. Gruppen oder Organisationen).

Solidarität ist für Honneth eine Anerkennungsform von sozialer Wertschätzung. Dabei ist sie nicht so stark objektivierbar, dass sie sich in Rechtsform gießen ließe und sie adressiert laut Honneth Fähigkeiten und Eigenschaften von Subjekten, weshalb sie sich auch von der Dimension Liebe unterscheidet (vgl. Honneth, 1994, S. 211). Solidarität ist eine Zuschreibung an Gruppenbeziehungen, deren ,intersubjektiver Werthorizont“ durch gemeinsamen Widerstand und die Fokussierung auf ein praktisches Ziel entsteht (Honneth, 1994, S. 208). Dies ist für Honneth allerdings eine historische Form von Solidarität, welche sich maßgeblich durch Individualisierung verändert. Voraussetzung für die von Honneth (1994) konstatierte ,posttraditionale Solidarität“ (S. 209) ist eine andere Form von Selbstbeziehung der Subjekte. Nicht mehr die Ausrichtung an einem gemeinsamen Ziel bewirkt die soziale Wertschätzung des/der Anderen, sondern ,die affektive Teilnahme am Besonderen der anderen Person“ ist hierfür notwendig (Honneth, 1994, S. 210). Diese normative Grundierung von Solidarität als einer intersubjektiven Form von Anerkennung verweist allerdings durchaus wieder in Richtung von organischer Solidarität im Sinne Durkheims. Gleichzeitig sind hier bereits jene Probleme angedeutet, mit denen sich subjektivierte Formen von Arbeit auseinandersetzen müssen (Bröckling, 2013) und sich u.a. in einer sukzessiven Entwertung von Würdigung als meritokratische Leitform zeigen (Voswinkel, 2012). Die Herstellung von Solidarität ist abhängig von den individualisierten Deutungshorizonten der Subjekte, da sie eines übergeordneten, kohärenten Ordnungs- (oder Wert-)bezugs entbehren. Eine entsprechende Deutungspraxis, was als solidarisch(es Handeln) gilt, bekommt hierdurch entscheidendes Gewicht. In der funktionalistischen Durkheimschen Fassung von Solidarität qua Arbeitsteiligkeit ist das individuelle Bewusstsein für die besonderen Leistungen und Fähigkeiten der Anderen (im Lichte der Eigenen) ausschlaggebend für dessen Aktualisierung. Nimmt man Honneths (1994) intersubjektive Fundierung hinzu, wird deutlich, dass auch das, was wir unter Solidarität bzw. solidarischem Handeln verstehen, Gegenstand von Aushandlung oder auch Kämpfen ist. Für Honneth wird Anerkennung auch erst durch tatsächliches Verhalten glaubwürdig und gewinnt damit Geltung (vgl. Honneth, 1994, S. 319). In diesem Sinne entsteht Solidarität durch affirmative Akte, die den/die Andere(n) intersubjektiv als solche(n) anerkennen (siehe auch Thijssen, 2012). Es liegt nahe, dass diese Affirmation nicht nur als kognitiver Akt angesehen werden kann, zumal es darum geht, 
spezifische Besonderheiten von Anderen positiv zu bestätigen. Eine emotionale Dimension dürfte daher in der Anerkennungspraxis in der Regel eine nicht unwesentliche Rolle spielen.

Wir gehen daher davon aus, dass auch digitale Solidarität unter Arbeitnehmer*innen von emotionaler Nähe als Katalysator/Verstärker von sozialer Kohäsion in solidarischen Kollektiven beeinflusst wird. Denn wie Hondrich \& Koch-Arzberger (1992) herausarbeiten, weist solidarisches Handeln über interessengeleitete Kooperation hinaus: „Gemeinsame Interessen in und an Arbeitsteilung führen nicht von selbst zur Solidarität, sondern nur dann, wenn sie kognitiv erkannt und emotional besetzt werden" (S. 19). Die emotionale Dimension ist ein wichtiges relationales Konstitutionsmerkmal von Solidarität, denn Zusammengehörigkeitsgefühle können die Tragweite solidarischen Handelns entscheidend beeinflussen. Emotionen scheinen jene Brücke zu bilden, die praktisch in der Lage ist, den latenten Widerspruch von ,identitär kodierten Subjekten im Singular und Plural“ bearbeitbar zu machen (Karakayali \& Moebius, 2014, S. 62) und damit auch Anerkennung im Sinne Honneths zu ermöglichen.

Den theoretischen Teil zusammenfassend, enthält unser Definitionsvorschlag für ,digitale Solidarität" (mindestens) folgende drei Dimensionen, die jedoch empirisch sehr unterschiedlich ausgeprägt sein können:

Erstens herrscht ein relativ hohes Maß an Differenz zwischen den Mitgliedern, übereinstimmend wird lediglich ein (abstraktes) Zugehörigkeitsmerkmal und wechselseitige Abhängigkeit hinsichtlich des Zielhorizonts vorausgesetzt.

Zweitens begegnen sich Zugehörige in einer (kommunikativen) Praxis reziproker Anerkennung, die Unterschiedlichkeit nicht nur toleriert, sondern dieser Respekt oder sogar Wertschätzung entgegenbringt.

Drittens werden solidarische Beiträge und Interaktionen von affektiv-emotionalen Momenten begleitet, die über rationalistische Begründungen von Kooperation hinausgehen.

Moderne, mehrdimensionale Solidarität entsteht also aus der emotional aufgeladenen Anerkennung von Differenz, welche gleichzeitig generalisierte Zugehörigkeit und eine universalistische Handlungsorientierung impliziert. Wie diese sehr voraussetzungsvolle Konzeption von Solidarität ihre Entsprechungen in digital mediatisierten Arbeitswelten finden kann, wird im folgenden Abschnitt diskutiert.

\section{Formen digitaler Solidarität}

Der gewerkschaftliche Diskurs zur Digitalisierung ist nicht selten von skeptischen bis pessimistischen Einschätzungen hinsichtlich der Auswirkungen für die Arbeitnehmerseite geprägt, und das betrifft auch die arbeitspolitischen Aspekte zur weiteren Entwicklung von Kollektivorganen der Interessenvertretung sowohl auf der tariflichen als auch auf der betrieblichen Ebene. Digitaler Technik wird dabei eine generelle Vereinzelungs- und Entsolidarisierungsdynamik unterstellt. Gemessen am traditional-mechanischen Solidaritätsmodell einer einheitlichen (aber wie gezeigt auch stark partikularistischen) Arbeiter- oder Klassenidentität, das nach wie vor als Hintergrundsemantik strukturbildend für gewerkschaftliche Strategien zur Mitgliedergewinnung und -bindung wirkt, könnten digital vermittelte In- 
teraktionen weder solidarische Gruppenzusammenhänge schaffen noch den notwendigen Verbindlichkeitsgrad unter Arbeitnehmer*innen gewährleisten, um z.B. für Arbeitskampfmaßnahmen zu mobilisieren. Der analoge, persönlich und face-to-face hergestellte und immer wieder auf diese Weise bestätigte Zusammenhalt von Beschäftigten und im Verhältnis zu ihren betrieblichen und/oder gewerkschaftlichen Repräsentanten gilt als Königsweg zur Genese von Solidarität. Unverbindlicheren und kurzfristigeren Konstellationen wird ein solidarischer Charakter von vorneherein abgesprochen. Die vorschnelle Absage an mögliche alternative Solidaritätsentwicklungen in digitalen Interaktionsräumen im Kontext der Arbeitsbeziehungen, der wir in unserer bisherigen explorativen Empirie sowohl bei Gewerkschaften als auch bei Betriebsräten begegnet sind, ist unserer Einschätzung nach jedoch fragwürdig. Denn sehr wohl lassen sich soziale Beziehungen unterschiedlichsten Formats digital stiften und auch über längere Zeiträume stabilisieren (siehe Klemm \& Staples, 2015, 2018). Dementsprechend sollten auch Solidaritätsverhältnisse in den Arbeitsbeziehungen digitalisiert möglich sein, auch ohne den Verlust ihrer emotionalen Bindungskraft (Pasquier $\&$ Wood, 2018). Wo und in welcher Form allerdings entstehen digitale Solidaritätsverhältnisse? Felix Stalder $(2013,2016)$ arbeitet vier Typen von modernen Solidaritätsformen heraus, die erst durch digitale Technologie voll zum Tragen kommen. Darum werden diese Formate digitaler Solidarität im Folgenden beschrieben und jeweils in Bezug zu den zuvor theoretisch formulierten Annahmen zur digitalen Solidarität gesetzt.

\subsection{Commons}

In digitalen Communities, die als Commons bzw. Allmenden organisiert sind, wird ein gemeinsamer Ressourcenpool von einem definierten und oft limitierten, prinzipiell gleichberechtigten und freiwillig zusammengeschlossenen Teilnehmerkreis ,bewirtschaftet". Commons-Mitglieder sind sowohl Produzent*innen als auch Konsument*innen. Im Unterschied zu herkömmlichen Commons handelt es sich in digitalen Communities in der Regel um immaterielle bzw. informationelle Ressourcen, d.h. sie verbrauchen sich nicht durch ihren Gebrauch und die erzeugten Produkte zählen zu den nicht-rivalen Gütern, d.h. deren Nutzung schränkt die Verfügungsoptionen für andere nicht ein. Die Handlungskoordination erfolgt weder über monetäre Anreize bzw. Preissignale (Markt) noch über Anweisung qua formalisierter Befehlsketten (Hierarchie). Oftmals bilden sich jedoch informelle Machtstrukturen heraus, die an meritokratischen Prinzipien orientiert sind: Diejenigen, welche am meisten Beiträge liefern, dürfen mehr über die Entnahme, das Management und die Entwicklung der Ressourcen und Güter entscheiden. Existenziell wichtig für den stabilen und dauerhaften Fortbestand von digitalen Commons ist es, ausreichend Beiträge zu generieren. Die wesentliche Herausforderung für digitale Commons stellt damit nicht Übernutzung dar (Ostrom, 2008), sondern die fortlaufende Sicherstellung von Beiträgen. Daher rührt auch die Bereitschaft, beitragsstarken Mitgliedern mehr Autorität zuzugestehen - ohne die Voraussetzung einer grundsätzlich gleichberechtigten common-based peer production in Frage zu stellen. Konflikte und Meinungsverschiedenheiten treten dennoch regelmäßig auf, die Selbststeuerungsfähigkeit von Commons ist daher auf praktikable Mechanismen der Konfliktbearbeitung und Entscheidungsfindung verwiesen. Leitend ist dabei die Vorstellung möglichst offen koordinierter und auf Konsens hinauslaufender Verständigung (analog zur Habermas 'schen kommunikativen Vernunft im herrschaftsfreien Diskurs), allerdings prag- 
matisch über informelle, meritokratisch legitimierte Hierarchien beschleunigt und handhabbar gemacht: Programmatisch ist das Zitat des Internetpioniers David D. Clark „We reject: kings, presidents and voting. We believe in: rough consensus and running code". 5 Zentraler Punkt zum Thema offener Koordinierung und grober Konsensfindung ist die besondere Bedeutung digitaler Kommunikationsformate: Erst durch internetbasierte peer-to-peer-Plattformen entsteht eine kommunikative Infrastruktur, mit der eine relativ große Anzahl von Teilnehmer*innen, unabhängig von räumlicher Kopräsenz, an Konsensfindungs- und Entscheidungsprozessen partizipieren kann.

Commons, insbesondere digitale Commons, entstehen nicht im luftleeren Raum, sondern sie benötigen ein bestimmtes Milieu, um existieren zu können. ${ }^{6}$ Bei Stalder sind gleicher Ressourcenzugang und ein geteilter kultureller Horizont hierfür eine Voraussetzung (Stalder, 2016, S. 35). Die theoretisch konstruierten Grundzüge digitaler Solidarität können am Beispiel von Wikipedia gut nachgezeichnet werden: Erstens stellt die Differenz hinsichtlich der einbezogenen Wissensdomänen das zentrale Kriterium für eine globale Enzyklopädie dar, wobei der gemeinsame Nenner die relativ universalistische Voraussetzung darstellt, ein humaner Wissensträger mit Internetanschluss zu sein. Der geteilte Zielhorizont, einen umfassenden Zugang zu allen, permanent aktualisierten Wissensgebieten zu schaffen, verweist unmittelbar auf die Interdependenz aller Partizipierenden. Zweitens bieten die offenen Diskussionsforen zu den Einträgen einen Raum für die freie Autorenschaft, sich kontinuierlich und transparent zu den Wissensinhalten auszutauschen und damit in nicht formal hierarchischer Weise Anerkennungsverhältnisse in der diskursiven Praxis zu konstituieren. Drittens zeigen sich auch emotional-affektive Komponenten, wie die leidenschaftlich geführten Debatten um die Formulierung der Artikeleinträge vermuten lassen, insbesondere aber am (unbezahlten) Engagement der ,Wikipedianer*innen' für die Wikipedia-community abzulesen ist. ${ }^{7}$

Digitale Commons ziehen ihre Kraft aus der Reziprozität und der Horizontalität einer potenziell äußerst breiten Beteiligung, was für die spätere Diskussion die Frage aufwirft, inwiefern diese Grundorientierung Vorbildcharakter für die institutionalisierte Mitbestimmung haben kann (oder sich bereits in ihr wiederfindet).

\subsection{Assemblies}

Assemblies werden von Stalder im Vergleich zu den Commons als kurzfristigere und spontanere Vergemeinschaftungsform beschrieben, meist im Rahmen sozialer Protestwellen, die als physische Zusammenkünfte entstehen (wie z.B. die Occupy-Bewegung), grundsätzlich aber auch als digitale Communities existieren können. Dabei orientiert sich die Teilhabe weniger an der gemeinsamen Bewirtschaftung von Ressourcen, meist stehen politische und soziale Anliegen im Zentrum. Hinsichtlich der Koordinations- und Entscheidungsverfahren

5 Die dem Zitat zugrundeliegende Originalpräsentation ist hier zu finden:

https://groups.csail.mit.edu/ana/People/DDC/future_ietf_92.pdf

6 Allerdings okkupieren, korrumpieren oder behindern die Prinzipien der Marktsteuerung als auch politischhierarchische Regime die freie Entwicklung von Commons. Insbesondere an der Entwicklung Wikipedias und ihrer Autorencommunity können diese Herausforderungen nachvollzogen werden (vgl. Jemielniak, 2014).

7 Siehe: https://de.wikipedia.org/wiki/Wikipedia: Wikipedianer. 
stehen Assemblies den Commons sehr nahe, allerdings mit einem expliziten Fokus auf die besonderen Schutzrechte für Minderheiten. In der Tradition von partizipativen Demokratieideen stehend, richten sich die Verfahrensregeln gegen die Fallstricke direkter Demokratie im Sinne einer Diktatur der Mehrheit. Wie in den Commons vermeiden Assemblies daher in der Regel Wahlverfahren zur internen Abstimmung, favorisiert wird dagegen der komplexe und meist langwierige Weg über eine diskursive Konsensbildung. Denn Mehrheitsentscheidungen sind zwar kurzfristig effizienter, können aber später höhere Monitoringkosten nach sich ziehen. Der angestrebte Schutz vor Diskriminierung wird in den Diskursregeln (culture of instant feedback, human mic, progressive stack, easy shift between roles of speaker and listener) deutlich (Stalder, 2013, S. 37-38). Übertragen auf OnlineAssemblies zeigen sich die spezifischen Organisationsprinzipien als Kultur der Transparenz und flexibler Partizipation. Der freie Zugang und die freiwillige Beteiligung gelten als zentrale Merkmale für kooperative Netzaktivitäten, wie sie exemplarisch in Open-SourceProjekten zum Ausdruck kommen. In Online-Assemblies schrecken weniger die höheren Kontrollkosten vor Mehrheitswahlentscheidungen ab, sondern die leichte Exit-Option der Überstimmten. Möglichst großer Konsens sichert somit eher das Überleben von Assemblies als Netzcommunities ab.

Unser Modell von digitaler Solidarität findet sich in Assemblies folgendermaßen wieder: Erstens spiegelt sich das Thema der Differenz in der Grundausrichtung dieser Versammlungsform wieder, Diversität wird durchgängig positiv besetzt und das Recht auf Verschiedenheit explizit betont. Entsprechend wenig spezifiziert stellt sich das einende initiale Anliegen dar, d.h. kollektive Positionierungen und Forderungen verbleiben meist auf einem relativ allgemeinen Niveau. Dafür wiegt die Wahrnehmung wechselseitiger Abhängigkeit deutlich schwerer, da die Vetomöglichkeiten von Einzelnen oder Wenigen beträchtlich sind. Zweitens drückt sich in der klaren Ablehnung jeglicher Diskriminierungspraxis der Anspruch an wertschätzender Anerkennung gegenüber Andersartigkeit aus. Drittens kann davon ausgegangen werden, dass die in den Assembly-Verfahren angelegte Überwindung von Vorurteilen sowie der wechselseitigen Perspektivenübernahme bei Erfolg zu besonders starken emotionalen Bindungseffekten führen.

Assemblies können als demokratietheoretisches Experimentierfeld angesehen werden, da in ihnen versucht wird, kollektive Handlungs- und Strategiefähigkeit zu generieren, ohne sich auf übliche Machtmechanismen zur Effizienzsteigerung zu stützen. Die transparenzorientierten Ansätze der Piratenpartei können als ein wohl gescheiterter Versuch gewertet werden, diese basisdemokratischen Ideen in eine parteipolitische Form zu gießen (Ringel, 2017). Wie in der späteren Diskussion noch aufzugreifen sein wird, stellt sich für Organisationen der institutionalisierten Interessenvertretung dennoch die Frage, wie sie mit den (steigenden) Ansprüchen an ,echter' demokratischer Beteiligung im Kontext sozial diverser Protestbewegungen konstruktiv umgehen kann oder will.

\subsection{Swarms}

Schwärme oder auch der Ausdruck ,Schwarmintelligenz' oszillieren zwischen den emanzipatorischen, selbstorganisierten Möglichkeiten digitaler Kommunikation und opaken anderen Formen von digitaler Kooperation. Interessant an Schwärmen ist ihr scheinbares Entstehen aus dem Nichts, ihre Emergenz, das dann sehr wohl konzertierte, massenhafte Han- 
deln - bis sie wieder zerfallen. Für Stalder allerdings unterscheiden sich Schwärme qualitativ von anderen Massenphänomenen insofern als er Schwärmen „reason“ (Vernunft) zuschreibt und anderen massenhaften sozialen Phänomenen „emotions“ und damit eher unoder vorbewusstes Handeln (Stalder, 2013, S. 41). Schwärme verfolgen ein explizites Handlungsziel und koordinieren sich daraufhin. Damit ist z.B. das bekannte Phänomen des Shitstorms kein Schwarm im Sinne Stalders. Zwar gibt es einen Anlass, der den Shitstorm entfacht, die Aktionen sind jedoch in der Regel weder koordiniert noch auf ein spezifisches Ziel gerichtet.

Der Social Media Forscher Clay Shirky hat drei grundlegende Bedingungen identifiziert, welche erfüllt sein müssen, um das Entstehen eines Schwarms zu ermöglichen. Sie schaffen erst das entsprechende Milieu. Mit „Promise, Tool und Bargain“ bezeichnet er diese Randbedingungen (Shirky, 2009, S. 260-261). Promise entspricht dabei dem zu verfolgenden Ziel oder zu lösenden Problem, Tool stellen die dafür nötigen Ressourcen dar (worunter auch Kommunikationsplattformen fallen) und mit Bargain sind jene Zugeständnisse adressiert, die die einzelnen ,Mitglieder' eines Schwarms leisten müssen, um den kollektiven Handlungsraum aufzuspannen (wie z.B. Kommunikationsregeln). Der für Stalder springende Punkt ist, dass die Individuen eben nicht im Kollektiv aufgehen, eben keine einheitliche Masse bilden, sondern dass durch die besondere Form der (temporären) Kooperation ,a new form of collective subjectivity without individual identity“ entsteht (Stalder, 2013, S. 41).

Digitale Solidarität zeichnet sich in Swarms dadurch aus, dass schon aufgrund der hohen Anzahl spontaner, individueller Schwärmlinge von mehr divergierenden als übereinstimmenden Merkmalen in der Teilnehmerschaft ausgegangen werden kann. Als emergente Phänomene weisen Swarms im Anlass und hinsichtlich der Zielorientierung nur punktuelle gemeinsame Schnittmengen auf. Die relativ schwach ausgeprägte Interdependenz im Schwarm zeigt sich in dessen flüchtiger und wenig verbindlicher Teilnahmestruktur. Zweitens ergibt sich gerade aus dem diffusen, dynamischen Teilnahmefeld und den geringen Einstiegshürden eine hohe Bereitschaft, anderen ,Mitstreiter*innen“ ungeprüft Anerkennung zu gewähren und (eng am Schwarmanlass und -ziel) Kooperationsbeziehungen auszuhandeln. Drittens deutet der spontan-impulsive Charakter von Swarms darauf hin, dass sowohl ihre Entstehung als auch deren (verhältnismäßig kurzes) Fortbestehen zu großen Teilen von der emotionalen Bewegtheit ihrer Schwarmmitglieder getragen werden.

Swarms sind in besonderer Weise auf die kommunikative Infrastruktur des (freien) Internets verwiesen, um die beschriebene virale, in ihrer Dynamik kaum plan- oder steuerbare Qualität solidarischer Kooperation zu entfalten. Insofern dürften Schwärme die originärste Form von digitaler Solidarität sein, da diese Art von Subjektivität ohne digitale Kommunikationstechnologie schwerlich vorstellbar ist. Der letzte Typ digitaler Solidarität ist ebenfalls tief in der digitalen Welt verankert.

\subsection{Weak Networks}

Im Unterschied zu Swarms, Commons und Assemblies sind weak Networks durch eine noch höhere Ambiguität bezüglich ihres emanzipatorischen Potenzials gekennzeichnet. Die gegenwärtig so erfolgreichen Social Networks (allen voran, das unvermeidliche Facebook) sind empirische Phänomene um die von Mark Granovetter (1973) so folgenreich beschrie- 
benen weak ties in ihrer Wirkung zu beobachten. Jenseits der grundsätzlich globalen Reichweite von weak Networks ermöglichen sie es den Nutzer*innen ,,[to] organise one's personal life as well as a means to engage with the world at large and to remake the world according this changed baseline of personal experience" (Stalder, 2013, S. 43-44). Entgegen der Klage über Echokammern und Filterblasen zeigt Stalder damit an, dass ,weak Networks' die Wahrnehmung von Welt verändern können. Und zwar durch die Möglichkeit von eng miteinander gekoppelten Gruppen, sich über ihre symbolischen Grenzen hinweg zueinander in eine Beziehung zu setzen (Stalder, 2013, S. 45). Dies entspricht der Intention von Granovetter, was die Leistung von ,weak ties“ angeht: „The significance of weak ties, then, would be that those which are local bridges create more, and shorter, paths" (Granovetter, 1973, S. 1366). Durch diesen Effekt kann Information, Wissen, aber auch symbolische Orientierung eine deutlich größere Verbreitung erreichen und zu informellen Formen von Handlungskoordination führen, wie z.B. im arabischen Frühling geschehen (obwohl in diesem Beispiel die Überschneidungen zum Schwarm sehr hoch sind). Die gegenwärtig erfolgreichen Netzwerke sind allerdings auch davon geprägt, dass sie für die einen eine Plattform sozialen Austausches und der Koordination sind, für die Anderen allerdings ein Geschäftsmodell. Insofern ist die grundsätzlich mögliche solidarische Form von weak Networks in hohem Maße von ökonomischen Interessen überformt. Nichtsdestotrotz können auf der Grundlage der bereitgestellten Infrastruktur des sozialen Netzwerks stabile, nichtkommerzielle Formen der Handlungskoordination erreicht werden, wenn auch der Beteiligungsgrad einzelner stark variieren mag. Dies muss allerdings kein Nachteil sein. Gerade der nur limitierte Zugang zum Gesamtbestand an Information in einem Netzwerk erlaubt es, dass diese mit erheblicher Wissensheterogenität umgehen können (vgl. Stalder, 2013, S. 44). Schwach sind diese Netzwerke für Stalder aber noch unter dem Aspekt, dass ihre Architektur in der Regel nicht von den Nutzer*innen überblickt oder gesteuert werden kann und dass die ökonomischen Interessen der Architekturinhaber*innen den Informationsaustausch im Netzwerk beeinflussen können (Stalder, 2013, S. 48; Lanier, 2014).

Jenseits der kommerziellen Interessen von Facebook wurde und wird das ,FreundeNetzwerk' jedoch in vielfältiger Form als digitale Infrastruktur genutzt, um Gruppenzusammenhänge mit solidarischem Kommunikations- und Interaktionscharakter zu schaffen. Als Beispiel können die Facebookgruppen der Clickworker*innen gelten, die auf der Amazon-Plattform Mechanical Turk um Arbeitsaufträge konkurrieren. ${ }^{8}$ Die drei Dimensionen digitaler Solidarität finden sich ebenfalls wieder: Erstens ist die Gruppenzusammensetzung meist äußerst heterogen, als Zugehörigkeitskriterium genügt es, auch Turker*in zu sein und der gemeinsame Zielhorizont besteht im Versuch, die Arbeitsbedingungen für Clickwork im konkreten Arbeitskontext, zum Teil aber auch strukturell zu verbessern. Zweitens zeigt sich in den Unterstützungsangeboten und Hilfestellungen in den Gruppen ein hohes Maß an gegenseitiger Anerkennung, obwohl die Wettbewerbssituation zwischen den Turker*innen offensichtlich ist. Drittens verbinden sich mit geteilter Arbeitsrealität offenbar Zusammengehörigkeitsgefühle, die z.B. in Gruppenbezeichnungen wie TurkerNation deutlich werden,

8 Einen Einblick in eine solche Gruppe haben wir durch ein exploratives Experteninterview mit einer ehemaligen Clickworkerin im Rahmen des oben genannten Forschungsprojekts erhalten. Eine ausführlichere Darstellung dieses empirischen Datums muss jedoch an anderer Stelle erfolgen. 
die aber auch in den alltäglichen Gruppenchats während der Arbeit emotionale Verbundenheit ausdrücken.

Die vier dargestellten Formen digitaler Solidarität leiten sich aus empirisch beobachtbaren Formationen in digitalen Kontexten ab, die von Stalder reklamierte Rekonstruktion von Idealtypen (im Weberschen Sinne) kann aufgrund der teilweise großen Schnittmengen zwischen den Typen jedoch kaum überzeugen. Insbesondere Commons und Assemblies weisen in den Organisationsprinzipien und beim Operationsmodus kaum Differenzen auf. Und auch zwischen den relativ losen, latent gehaltenen und deshalb potenziell besonders wirksamen Kooperationsbeziehungen in Swarms und weak Networks zeigt sich kein wesentlicher Unterschied in der zugrundeliegenden Solidaritätslogik. Der Sachbezug unterscheidet diese beiden Formen. Schwärme organisieren sich rund um ein Promise und lösen sich bei Erreichen oder Scheitern wieder auf, weak Networks hingegen können in der Zeit unterschiedliche Ziele bearbeiten. Das übergeordnete ,Ziel` ist es vielmehr die gruppentranszendierenden Verbindungen aufrechtzuerhalten. Nichtsdestotrotz kann Stalders Typologie herangezogen werden, um die Frage nach der Herstellung von (mitbestimmungsrelevanter) Solidarität in einer sich digitalisierenden Arbeits- und Lebenswelt zu bearbeiten. Relevante Dimensionen zur Identifikation und Unterscheidung von Solidaritätsformen stellen demnach die Dauerhaftigkeit und Stabilität digitaler Communities dar, der Grad (gefühlter) Verbundenheit ihrer Mitglieder sowie das entsprechende Ausmaß von Commitment und Verbindlichkeit. Ebenso scheinen Gruppengröße und die Interaktionsdichte bedeutende graduelle Unterschiede zu markieren und schließlich verweisen diverse Kollaborations- und Kooperationsweisen sowie die jeweiligen (politischen) Steuerungs- und Koordinationsverfahren auf Variationen von digitaler Solidarität.

Gemeinsam ist allen digitalen Solidaritätsformen jedoch, dass sie innerhalb des konzeptionellen Rahmens von organischer Solidarität operieren. Hierfür spricht die entsprechend größere Diversität/Heterogenität der Partizipierenden, die Anerkennung individueller Autonomie im Kollektiv als Wert für sich und nicht hierarchische und in der Regel nicht kommerzielle Kooperations- und Koordinationsmodi. IKT-Technologien stellen eine digitale Infrastruktur zur Verfügung, die ortsunabhängige, egalitäre und transparente Vergemeinschaftungsprozesse für eine sehr große Anzahl von Partizipierenden ermöglicht. Wobei hier ebenfalls begriffliche Vorsicht angebracht ist, denn Vergemeinschaftung in einem klassischen Weberschen Sinn, liegt bei den diskutierten Solidaritätsphänomenen eher nicht vor (Hanke \& Morlok, 2015, S. 1471). Dafür sind sie zu reflexiv angelegt und legen zu viel Wert auf die Autonomie des/der Einzelnen. Gleichzeitig ist Zugehörigkeit nicht nur interessengesteuert, sondern insbesondere von einem subjektiven Zugehörigkeitsgefühl abhängig.

Aus den genannten Merkmalen ergeben sich direkte Anschlussmöglichkeiten für die Frage, wie Arbeitnehmer*innen und die kollektive Organisation ihrer Interessenvertretung vor dem Hintergrund der Digitalisierung den Herausforderungen begegnen können, neue bzw. veränderte Solidaritätspotenziale anzuerkennen und arbeitspolitisch zu nutzen. 


\section{Digitale Solidarität in den industriellen Beziehungen der heutigen Arbeitswelt?}

Welche Relevanz haben die verschiedenen Formen digitaler Solidarität für die arbeitspolitische Gestaltung der industriellen Beziehungen? Wo deuten sich Formen digitaler Solidarität an und inwiefern verweist dies auf einen Wandel in der Arbeitnehmervertretung/Mitbestimmung, die ihre legitimatorische Grundlage maßgeblich aus der Referenz auf Solidarität und solidarisches Handeln bezieht?

Unsere theoretische Skizze zu den drei Dimensionen von digitaler Solidarität und die Reflexion auf die digitalen Solidaritätsformen von Stalder, die originär oder mittelbar mit digitaler Kommunikationstechnologie zusammenhängen, sollen im Folgenden ins Verhältnis zu aktuellen arbeitspolitischen Herausforderungen gesetzt werden. Gleichzeitig gehen wir auch davon aus, dass digitale Solidarität nur Bindungskraft entwickeln kann, wenn sie empirisch sowohl die theoretisch ausgearbeiteten Dimensionen mit Leben füllen kann, als auch die von Stalder explizierten Organisationsprinzipien aufweist. Damit hängt die pragmatische Frage zusammen, wie Akteure der Mitbestimmung mit einem Abnehmen der Bindungskraft von mechanischer Solidarität umgehen können, indem sie die hier vorgestellten Formen digitaler Solidarität als relevante Orientierungsgröße in die Interessenpolitik/Mitbestimmung einfließen lassen. Hierfür diskutieren wir drei bekannte Veränderungskomplexe der Arbeitswelt: Die Heterogenisierung der Workforce, die zunehmende Auflösung von einem einheitlichen Ort der Arbeit und die Intensivierung von Konkurrenzbeziehungen unter Arbeitnehmer*innen. Diese Veränderungsphänomene sind nicht erschöpfend, aber unserer Ansicht nach beispielhaft für die aktuellen und potenziellen Veränderungen der Organisation von Arbeit. Digitalisierung ist für keines dieser Phänomene als Ursache zu werten, vielmehr sind hier die Stichworte Globalisierung, Individualisierung und Finanzialisierung anzuführen (Stichweh, 2000; Hall \& Soskice, 2001). Digitalisierung kann hier allerdings ungemein ermöglichend oder verstärkend wirken und gravierende Nebenfolgen zeitigen, wenn man an die Ideen und Umsetzungen der Sharing Economy denkt.

\subsection{Heterogenisierung der Arbeitnehmer*innen}

Unübersehbar hat sich die Workforce in den letzten drei Jahrzehnten massiv diversifiziert: eine gestiegene Erwerbsbeteiligung von Frauen, mehr Arbeitnehmer*innen mit Migrationshintergrund, die Pluralisierung von Berufsbildern und nachgefragten Kompetenzen - um nur einige Merkmale der heterogener gewordenen Beschäftigtenstruktur zu nennen. Das sogenannte Normalarbeitsverhältnis und der implizit damit mitverstandene Mann sind keinesfalls mehr der faktische, zum Teil jedoch noch der normative Standard für die Arbeitskraftseite eines Erwerbsverhältnisses (Hinrichs, 1996; Bosch, 2002). Diese Pluralisierung bringt allerdings eine Differenzierung oder Partikularisierung von Interessen mit sich, die Solidarität als eine normative Ressource für die Mitbestimmung schwächen (Helfen \& Nicklich, 2014). Diversität wird zwar gegenwärtig als eine neue Ressource adressiert. Organisationen haben entsprechende Stellen ausgebildet, die auf eine ausreichende Diversität der Belegschaften achten sollen und die deren Produktivität in die organisationalen Selbstbeschreibungen einfließen lassen (Schulz, 2009). Neben der ökonomisch relevanten Frage, 
welche Diversitätsdimensionen als Ressource im Betrieb zu erachten sind, stellt sich diese Frage auch, wenn es um Solidarität und ihre Aufrechterhaltung geht. Zwar verfügen einige traditionsreiche Industriekonzerne noch über genügend Identifikationspotenzial, um ihre heterogener werdenden Belegschaften einzuhegen, mindestens aber der Graben zwischen kollektiv orientierten blue collars und individualisierten white collars kann damit nicht überbrückt werden. Ebenso wird in diesem Zusammenhang nach wie vor die Macht von Kultur unterschätzt. In einer Studie zur Solidarität zur Mitbestimmung auf der europäischen Ebene von Unternehmen zeigen die Autor*innen, dass der Verweis auf prinzipiell gleiche Interessen bei weitem nicht ausreicht, um Solidaritätsbekundungen in solidarisches Handeln umzumünzen. Die Autoren machen sich allerdings dafür stark, dass nicht partikulare Interessen für diese Diskrepanz verantwortlich sind, sondern divergierende kulturelle Konzepte von Solidarität (Klemm, Kraetsch \& Weyand, 2011, S. 300). Die Spezifika des deutschen Modells betrieblicher Mitbestimmung statten ihre Vertreter mit Machtpositionen aus, welche eng mit einer Verpflichtung auf das Allgemeinwohl verknüpft sind. „Prinzipien und Regeln der Organisation von Solidarität sind so, trotz aller Veränderungen, abgesichert und werden von den Spitzen der Gesamtbetriebsräte gleichsam verkörpert" (Klemm, Kraetsch \& Weyand, 2011, S. 305). Dieser Konnex ist allerdings brüchig geworden und die Annahme, dass Diversität und kulturelle Orientierungen auf Milieuebene hierbei eine nicht zu unterschätzende Rolle spielen, scheint nicht weit hergeholt. Gerade hier können allerdings digitale Solidaritätsformen ihre Stärken zeigen. Commons setzen auf die selbstorganisierte Eigenleistung, die ,Beiträge' der Einzelnen. Die zu erhaltende Allmende wäre dann die Interessensvertretung selbst. So zeigen sich kollaborative Netzwerke und insbesondere „Neogemeinschaften“ erstaunlich stabil gegenüber Heterogenität und daraus entstehenden Meinungsunterschieden, da die Binnenkommunikation Homogenisierung forciert und alle Beteiligten auf den gleichen Horizont orientiert sind (Reckwitz, 2017, S. 275-276). Für ein produktives digital Organizing stellt sich dann die Aufgabe für eine hinreichend egalitäre Grundstruktur zu sorgen, den gemeinschaftlichen Grundgedanken affektiv aufzuladen und dennoch Anschlussmöglichkeiten für andere weak Networks bereitzuhalten.

\subsection{Dislozierung der Arbeitnehmer*innen: Digitale Kommunikationsmedien in virtuellen Interaktionsräumen}

Betriebsvereinbarungen zum mobilen Arbeiten stehen symptomatisch für die Herausforderungen, die die digitale Transformation der Arbeitswelt an ihre Regulierung stellt. Wie kontrolliert man Arbeitszeit, wenn die Beschäftigten nicht mehr sichtbar vor Ort sind und wie schützt man sie vor Ausbeutung? Hinter diesen praktischen Fragen steckt das Problem der Adressierbarkeit. Für die Mitbestimmungsvertreter*innen heißt das, wie können sie sicherstellen, dass Kolleg*innen, die in der Regel nicht leiblich ko-präsent sind, sich doch gemeint fühlen und damit auch aktive Mitglieder der Belegschaft sind? ${ }^{9}$ Wie geht man also um mit einer Belegschaft, die nicht nur vielfältiger, sondern auch unterschiedlich sichtbar ist? Erst einmal scheinen digitale Kommunikationsmedien ideal, um Distanzen zu überbrü-

9 In einer gewissen Weise geht es darum, wie weiterhin sichergestellt werden kann, dass Beschäftigte Subjekte der Mitbestimmung werden können, wenn sie einer typischen Anrufung durch leibliche Nicht-Anwesenheit entzogen sind. 
cken. Ortsunabhängig können Kolleg*innen adressiert werden und Inhalte sind über bestimmbare Zeiträume für alle verfügbar. Bemerkbar macht sich an dieser Stelle sofort, dass die Binnendifferenzierung von Betrieben ungleich ist und sich bspw. dadurch zeigt, dass nur ein bestimmter Teil der Kolleg*innen überhaupt Zugang zu den digitalen Angeboten des Betriebs hat. Schwerwiegender scheint allerdings ein tieferliegendes Misstrauen gegenüber dem gemeinschaftsstiftenden Potenzial von digitalen Kommunikationsmedien. Prominente Autor*innen verweisen darauf, dass soziale Beziehungen, die nur oder hauptsächlich digital geführt werden, nicht dieselbe Qualität aufweisen, wie solche in leiblicher KoPräsenz (Turkle, 2011). Digitale Kommunikationsbeziehungen stehen unter dem Verdacht des Nicht-authentischen. Unbestreitbar aber haben digitale Kommunikationen Konsequenzen für die soziale Wirklichkeit, sei sie nun digital oder analog. Die Verwendung von skopischen Medien muss allerdings damit umgehen, dass ihnen leibliche Kopräsenz als wichtige Dimension in der Interaktion fehlt. Für Goffman (1983) war daher nur ko-präsente Interaktion eine solche in vollem Umfang. Der Körper fungiert in der Interaktion als ein Anzeiger und ausgleichendes Element, welches strategisch eingesetzt werden kann, aber vor allem in der vorbewussten Wahrnehmung des/der Anderen eine zentrale Rolle spielt (Garfinkel, 1964; Goffman, 1990). Digital mediatisierte Kommunikation ist vornehmlich textuell organisiert, d.h. qua objektivierter Sprache. Es wäre nun verfehlt anzunehmen, dass es schlicht an der Körperlichkeit mangelt. Die interessante Frage ist, wie Interaktionsteilnehmer*innen dieses Fehlen bearbeiten. Klemm \& Staples (2015) gehen davon aus, dass die körperliche Dimension in Text übersetzt werden muss. Implizite Anzeichen und Annahmen über das Gegenüber müssen nun expliziert werden, sei es durch Sprache oder die mittlerweile ubiquitären Piktogramme (Emojis). Diese Übersetzungsanstrengungen sind notwendig, da ansonsten die Interaktion von den Teilnehmer*innen unzureichend typisiert wird (Schütz, 2003), was dann zu mitunter gravierenden kommunikativen Nebenfolgen führt. Houben (2018) plädiert daher dafür, den Begriff der Kopräsenz durch Ko-Referenz zu ersetzen. Die Interaktionsanalyse würde hierdurch zeichentheoretisch erweitert und digital medialisierte Interaktionsformen könnten als ein Typ neben anderen untersucht werden. Von diesem Standpunkt aus wird deutlich, dass Solidarität auch mittels digitaler Kommunikation aktiviert werden kann. Mitbestimmung, die stärker auf Beteiligung setzt, kann das produktiv mit digitalen Kommunikationsmedien tun. Wenngleich eingeräumt werden muss, dass erfolgreiche digitale Kommunikation auch das Einüben neuer Kommunikationsstile erfordert, da die fehlende körperliche Dimension reflexiv mitgedacht werden muss. Mitbestimmungsakteure können damit sichtbar anerkennende Wertschätzung zeigen, auch durch den Umstand, dass dieser Typ von Kommunikation ungeschützter ist als bspw. mittels Betriebszeitung oder einer Rede. Dies wiederum stärkt die Legitimität von Mitbestimmung und macht solidarisches Handeln sichtbar.

\subsection{Zunehmende Konkurrenz zwischen Arbeitnehmer*innen}

Mit der v.a. ökonomischen Globalisierung und der zunehmenden internationalen Öffnung von Binnenmärkten verschärft sich der Konkurrenzdruck nicht nur für Unternehmen, auch Arbeitnehmer*innen stehen sich immer direkter und ungeschützter als Konkurrent*innen gegenüber. In den westlichen Industrieländern spüren dies die im globalen Vergleich relativ privilegierten Beschäftigten spätestens seit den 1990er Jahren durch die Verlagerung von 
Produktion ins Ausland (Statistisches Bundesamt, 2008). Inzwischen sind aber auch Dienstleistungsbereiche und hochqualifizierte Angestelltentätigkeiten betroffen (vgl. Boes \& Kämpf, 2011). Im Zuge wachsender Arbeitsmigration und speziell der EU-Arbeitnehmerfreizügigkeit in Deutschland seit 2011 sind viele Arbeitsmärkte noch kompetitiver geworden (Staples, Trinczek \& Whittall, 2013). Dabei stellt die zunehmende Wettbewerbsorientierung und Konkurrenzverschärfung innerhalb der Arbeitnehmerschaft auch die Gewerkschaften und andere Akteure kollektiver Interessenvertretung vor gravierende Integrationsund Solidaritätsprobleme. Angesichts konkurrierender Standorte innerhalb desselben Konzerns/Unternehmens, Kern- und Randbelegschaften im selben Betrieb (Holst, 2009) oder der Zersplitterung in Berufs- und Spartengewerkschaften (Schroeder, Kalass \& Greef, 2011) drängt sich die Frage auf, ob Solidarität unter Arbeitnehmer*innen jenseits von Partikularismen noch möglich bzw. organisierbar ist.

Allerdings ist Konkurrenz zwischen Arbeitnehmer*innen um Arbeitsplätze oder zwischen Beschäftigten um materielle und symbolische Privilegien (Gehalt, Karriereoptionen, Berufsprestige etc.) seit jeher integraler Bestandteil einer grundsätzlich als Markt organisierten Verteilung von Erwerbsarbeit (Hinz \& Abraham, 2008). Insofern erhält jede Assoziation von Arbeitskraft, egal ob sie als Betriebsversammlung der Belegschaft, als Berufsverband oder Gewerkschaft in Erscheinung tritt, einen konkurrenzmindernden Charakter. Solidarität tritt in dieser Perspektive demnach als Gegenspieler zum Wettbewerbsparadigma auf. Während enge Kooperation im Sinne von ,Klüngelei' als wettbewerbsverzerrend bzw. -schädigend gilt, wird übermäßiger Konkurrenzorientierung in Gestalt einer egoistischen ,Ellenbogenmentalität‘ generell eine solidaritätszersetzende Wirkung unterstellt. Insbesondere der mechanische Solidaritätstypus ist auf eine Invisibilisierung bzw. Verleugnung von Konkurrenzbeziehungen angewiesen, um den Anschein von symmetrischen Anerkennungsverhältnissen unter Gleichen aufrecht $\mathrm{zu}$ erhalten, die sich gegenseitig nichts wegnehmen wollen oder würden. Und auch im Falle der organischen Solidarität nach Durkheim führt die steigende Konkurrenz zwar zu weiterer sozialer Arbeitsteilung qua beruflicher Spezialisierung (weshalb die Interdependenz zunimmt), aber genau dadurch können Konkurrenten dem direkten Wettbewerb aus dem Weg gehen. Die Entstehung von Solidarität (egal in welcher Form) gilt daher unter den Bedingungen von offen zu Tage tretender Konkurrenz als extrem unwahrscheinlich (vgl. hierzu mit Rekurs auf Durkheim in Honneth, 2008, S. 339).

In den vorgestellten digitalen Solidaritätsformaten finden sich jedoch Anhaltspunkte, wie Kooperation und Konkurrenz zusammengedacht werden könnten, sich Arbeitnehmer*innen also nicht nur trotz, sondern auch wegen ihrer kompetitiven Interessen auf externen und internen Arbeitsmärkten solidarisieren. „Denkbar ist, dass sich soziale Netzwerke und Projekte als Felder der Zusammenarbeit und des Wettbewerbs und damit als Praxis einer Koopetition formen, die den traditionellen Gegensatz zwischen Kooperation und Kompetition hinter sich lässt" (Reckwitz, 2017, S. 218). Netzwerke haben dabei eine Doppelrolle; dadurch, dass sich Kooperationen durch Reziprozitätserwartungen verändern, können auch neue Solidaritätsformen entstehen (vgl. Windeler, 2012, S. 45).

Dabei geht es keinesfalls um eine begriffliche Synthese, wie das Kofferwort Koopetition (Jansen \& Schleissing, 2000) andeuten könnte, und ein entsprechend widerspruchsfreies Solidaritätskonzept, vielmehr bleibt die spannungsreiche Ambivalenz auf der theoretischen 
Ebene bestehen. Die konkrete Praxis digitaler Communities lässt jedoch einen aufgeklärten, insofern also entspannten Umgang von Wettbewerber*innen mit Kooperationspartner*innen und vice versa vermuten. Insbesondere bei den auf Dauer orientierten Solidaritätsformen der Commons und weak Networks scheint der Schatten zukünftiger Interaktionen wesentlich auf die Kooperationsbereitschaft zurückzuwirken, kurzfristige Wettbewerbsvorteile durch opportunistisches Verhalten gefährden dagegen eine nachhaltige Erfolgssicherung. Eine zentrale Rolle spielt zudem die umfassende Transparenz, die in digitalen Kommunikations- und Interaktionsräumen möglich wird. Defektierende oder aggressiv kompetitive Akteur*innen sind für andere Community-Teilnehmer*innen unmittelbar identifizierbar, der notwendige Vertrauensvorschuss für weitere interne Kooperationsoptionen würde schnell verfallen.

In Assemblies und Swarms sind solidarische Praxen der Fusionierung von Kompetition und Kooperation am besonderen Charakter der Konkurrenzbeziehungen angelagert. So hält bereits Georg Simmel für die Eigenart von Konkurrenz im anonymen Marktgeschehen fest, „dass der Kampf ein indirekter ist. (...) Die Form des Konkurrenzkampfes ist vor allem nicht Offensive und Defensive - deshalb nicht, weil der Kampfpreis sich nicht in der Hand eines der Gegner befindet“" (Simmel, 1992, S. 174). Die relative Anonymität in den digitalen Kollektiven von Assemblies und Swarms ermöglicht die Adressierung von kompetitiven Elementen (im Rahmen meritokratisch ausgetragener Kämpfe, z.B. um Wortführerschaft), ohne den solidarischen Horizont der gemeinsamen Willensbildung und Zielverfolgung zu verlassen. In Swarms sind Promise und Tools auf der Basis bestimmter Kommunikationsregeln (Bargain) keine feststehenden ideologischen Imperative, sondern variable Bestandteile kollektiver Identität, um die kompetitiv gerungen werden kann, ohne den digitalen solidarischen Kooperationszusammenhang einer ,gemeinsamen Sache“ deshalb aufzukündigen.

\section{Fazit: Perspektiven und Potenziale digitaler Solidarität für eine Arbeitspolitik 4.0}

Was folgt nun aus den dargestellten Formen digitaler Solidarität für die kollektive Organisation und Interessenvertretung von Arbeitnehmer*innen in den industriellen Beziehungen? Diese Frage kann u.E. aus (mindestens) zwei verschiedenen Perspektiven gestellt und im Folgenden für künftige Arbeitnehmersolidarität erörtert werden. Erstens haben sich in den verschiedenen Online-Communities der Commons, Assemblies, Swarms und weak Networks bereits auch konkrete Produktions- und Arbeitszusammenhänge im virtuellen Raum entwickelt, auch wenn der Anteil bzw. Charakter von (Erwerbs-)Arbeit sowie der Arbeitnehmerstatus der Beteiligten jeweils sehr unterschiedlich ausgeprägt sind. Hierbei wäre zu klären, inwiefern Gewerkschaften und Betriebsräte versuchen können, diese Formen digitaler Arbeit interessenpolitisch zu organisieren und dabei an die Beschaffenheit und das Potenzial digitaler Solidarität direkt anzuschließen (1.). Zweitens können kollektive Akteure der Interessenvertretung ausloten, inwiefern sie die verschiedenen Formate digitaler Solidarität auf sich selbst anwenden könnten, also im Rahmen einer digitalisierten Gewerkschaftsund Betriebsratsorganisation (Teile der) Operations- und Koordinationsmodi von Com- 
mons, Assemblies, Swarms und weak Networks adaptieren und zum Fluchtpunkt für ihre institutionelle Strukturierung machen können (2.).

$\mathrm{Zu}$ 1.) In digitalen Communities werden soziale Interaktionen (selbst-)organisiert, wobei sich neben rein kommunikativen auch kollaborative Strukturen herausbilden, die also auch als digitale Arbeitszusammenhänge aufgefasst werden können, in denen Wissen zirkuliert, Ressourcen bereitgestellt und genutzt werden, wodurch (kollektive) Güter und Dienstleistungen hergestellt werden. Diese Vorgänge können nicht als Epiphänomene einer umfassenden kapitalistischen Wirtschaftsordnung verstanden werden. Virtuelle Wertschöpfungsprozesse und Arbeitsleistungen, nebst digitalen Eigentumsverhältnissen und Rechtsauffassungen bzw. -verfassungen lassen sich zwar nicht unabhängig von der analogen Ökonomie begreifen, wie die unübersehbar fortschreitende Kommerzialisierung des Internets belegt, aber sich auch nicht darauf reduzieren. Entsprechend kann auch die darauf bezogene tarifliche und betriebliche Arbeitspolitik mit ihren institutionell gewachsenen Strukturen zur Gestaltung v.a. industrieller Arbeitsbeziehungen kaum adäquat auf digitale Kontexte passen. Als eigenständige und emergente soziale Gebilde widersetzen sie sich (nicht immer erfolgreich) sowohl einer marktlichen als auch einer hierarchischen Steuerung, Manipulation oder gar Übernahme von außen. Lassen sich Gewerkschaften und Betriebsräte jedoch auf die eigentümliche Funktionslogik digitaler Solidarformen ernsthaft ein, die sich aus den spezifischen Eigenarten digitaler Arbeit ,natürlich“ ergeben, können sie kaum in das Repräsentationsmodell klassischer Gewerkschafts- und Betriebsratspolitik eingepasst werden. Erste Ansätze einer Annäherung zwischen Gewerkschaften und Online-Communities existieren bereits, bspw. im Rahmen eines Workshops von verdi und der ,TurkerNation' ${ }^{10}$ In der Dokumentation der Veranstaltung deuten sich jedoch genau jene Kompatibilitätsprobleme an, die zwischen den digitalen vs. gewerkschaftlichen Solidaritätsmodellen angelegt sind: Die extreme Spannweite an Heterogenität seitens der Crowdworker*innen auf der einen Seite und der dominante Fokus auf Gemeinsamkeit qua Ähnlichkeit seitens der Gewerkschaft. Insbesondere im Rahmen des spezifischen institutionellen Settings industrieller Beziehungen in Deutschland erscheint es aus heutiger Sicht fraglich, ob Gewerkschaften und Betriebsrät*innen sich neuen Formen digitaler Solidarität systematisch öffnen werden. Gleichwohl werden Elemente wie eine (authentische) Partizipationskultur wohl entscheidend sein, um sowohl Legitimität gegenüber den Beschäftigten aufrechtzuerhalten, als auch den komplexen Aufgaben einer modernen kollektiven Interessenvertretung gerecht werden zu können.

$\mathrm{Zu}$ 2.) Die Frage, wie sich Gewerkschaften auf digitale Solidaritätsformen einstellen oder sogar daran anknüpfen können, ist nicht unabhängig von den Veränderungsfähigkeiten der eigenen Organisationsstrukturen und -operationen zu stellen. Damit ist zum einen der (technische) Komplex aus Internet-Präsenz und digitalen Kommunikations- und Interaktionsweisen in der konkreten Gewerkschafts- und Betriebsratsarbeit angesprochen. Zum anderen knüpft sich an die Übernahme von Organisationsprinzipien aus Commons, Assemblies, Swarms und/oder weak Networks ein hohes Maß an basisdemokratischen Partizi-

10 Das ver.di-Verbundprojekt „cloud und crowd“ hatte Anfang 2017 die Mitbegründerin von TurkerNation Kristy Milland eingeladen, um Kontakt- und Kooperationsoptionen zu Crowdworker*innen zu eruieren (vgl. https://innovation-gute-arbeit.verdi.de/ueber-uns/forschungsprojekte/cloud-und-crowd/++co++5a8263100f0d-11e7-a707-525400ed87ba). 
pations- und Diskursangeboten für (potenzielle) Mitglieder bzw. Beschäftigte, die dem hierarchischen und funktionärslastigen Repräsentationsmodell heutiger Einheitsgewerkschaften und Betriebsratsstrukturen eher widersprechen. Der (mehr oder weniger) zentralistische Aufbau klassischer Gewerkschaftsorganisationen und Betriebsratsgremien gerät mit der dezentralen Netzwerkstruktur der Internet-Kommunikation in Konflikt, da strategische Planungsvorgaben und Kontrollmöglichkeiten top-down nicht mehr durchzusetzen sind. Damit einher geht die Betonung des Anspruchs auf Selbstorganisation in digitalen Communities, der auch arbeitspolitisch mit hohen Autonomiefreiräumen zur Selbst(re)präsentation verbunden wird. Das bisher zugrundeliegende Primat kollektiver, konkurrenzreduzierender Interessenvertretung von Arbeitnehmer*innen wird somit in ihrem fundamentalen Solidaritäts- und Identitätsverständnis herausgefordert. Das Kollektiv scheint in digitalen Kontexten nur mehr vor dem Hintergrund des Individuums zu haben zu sein. Eine Umorientierung in dieser Richtung hätte für alle Organe der Interessensvertretung ernsthafte Konsequenzen. Sie wären dazu gezwungen, den Widerspruch zu bearbeiten, dass individuelle Interessen, Selbste im Fokus stehen, dass allerdings das Kollektiv notwendig und zentral dafür sein soll, dieser Form von Interessenvertretung gerecht zu werden. Für die von uns beschriebenen Phänomene digitaler Solidarität scheint diese Ambiguität keine Identitätskonflikte mit sich zu bringen. Im Gegenteil, daraus ziehen diese Netzwerke sowohl ihre produktive Kraft als auch ihren solidarischen Zusammenhalt.

Eine grundlegende Fragestellung gewerkschaftlicher Adressierung digitaler Solidarität kann hier nur angeschnitten werden: Welche Rolle könnten Gewerkschaften in und für digitale Commons wie Wikipedia spielen? Die freiwillige, unbezahlte Arbeit an einer globalen Enzyklopädie fällt trotz des hohen Nutzens für die Allgemeinheit nicht in den Zuständigkeitsbereich von Gewerkschaften, sofern sie sich ausschließlich für die Interessen abhängiger Erwerbsarbeit einsetzen. Streng genommen müssten Gewerkschaften sogar gegen Wikipedia vorgehen, weil andere Lexikonverlage und die daran hängenden Arbeitsplätze verdrängt werden. Andererseits könnten Gewerkschaften ihre Aufgabe auch dahingehend (um)definieren, lebendige Arbeit und deren Produktivkraft in all ihren Formen zu protegieren bzw. vor dem Zugriff kommerzieller Interessen zu schützen. Diese Überlegungen führen zu der Debatte über den Wert von Arbeit an sich - und ob auf der Grundlage digitaler Infrastrukturen solidarische Arbeits- und Lebenszusammenhänge entstehen könnten, die jenseits der kapitalistischen Verwertungs- und Profitorientierung Bestand haben können.

Die Entstehung und Weiterentwicklung digitaler Solidarität sehen wir insgesamt als einen nicht abgeschlossenen Prozess, in welchem Kooperationsmodi und meritokratische Normen neu verhandelt werden. Durch ihre Ambiguität im Verhältnis von Subjekt zu Kollektiv beinhaltet sie sowohl das Potenzial zu einer Erneuerung von Solidarität hin zu echter organischer Solidarität, aber auch das Risiko, gesellschaftliche Vereinzelungstendenzen zu befeuern.

\section{Literaturverzeichnis}

Beck, U. \& Lau, C. (2005). Theorie und Empirie reflexiver Modernisierung: Von der Notwendigkeit und den Schwierigkeiten, einen historischen Gesellschaftswandel innerhalb der Moderne zu beobachten und zu begreifen. Soziale Welt, 56 (2/3), 107-135. doi: 10.5771/0038-6073-2005-2-3-107

Boes, A. \& Kämpf, T. (2011). Global verteilte Kopfarbeit. Offshoring und der Wandel der Arbeitsbeziehungen. Berlin: edition sigma. doi: 10.5771/9783845269542 
Boes, A., Kämpf, T., Langes, B. \& Lühr, T. (2015). Landnahme im Informationsraum. Neukonstituierung gesellschaftlicher Arbeit in der „,digitalen Gesellschaft“. WSI-Mitteilungen, 68, 77-85. doi: 10.5771/0342-300X-2015-2-77

Bosch, G. (2002). Das Normalarbeitsverhältnis in der Informationsgesellschaft. Institut Arbeit und Technik: Jahrbuch, 2003, 11-24.

Bröckling, U. (2013). Das unternehmerische Selbst (5. Aufl.). Frankfurt am Main: Suhrkamp.

Brown, T. (2009). Change by design. New York, NY: HarperCollins.

Durkheim, É. (1977). Über die Teilung der sozialen Arbeit. Frankfurt a.M.: Suhrkamp.

Garfinkel, H. (1964). Studies of the Routine Grounds of Everyday Activities. Social Problems, 11 (3), 225-250. doi: 10.2307/798722

Gauntlett, D. (2007). Creative Explorations. New approaches to identities and audiences. Abingdon: Routledge.

Goffman, E. (1983). The Interaction Order: American Sociological Association, 1982 Presidential Address. American Sociological Review, 48 (1), 1-17. doi: 10.2307/2095141

Goffman, E. (1990). The presentation of self in everyday life. New York [N.Y.]: Doubleday.

Granovetter, M. S. (1973). The Strength of Weak Ties. American Journal of Sociology, 78 (6), 13601380. doi: $10.1086 / 225469$

Hall, P. A. \& Soskice, D. (2001). Varieties of capitalism. Oxford [u.a.]: Oxford Univ. Press. doi: 10.1093/0199247757.001.0001

Hanke, E. \& Morlok, C. (Hrsg.). (2015). Max Weber, Wirtschaft und Gesellschaft: Gesamtregister. Tübingen: Mohr.

Helfen, M. \& Nicklich, M. (2014). Gewerkschaften zwischen Konkurrenz und Kooperation? Interorganisationale Beziehungen in der Facility Services-Branche. Industrielle Beziehungen, 21 (2), 181-204. doi: 10.1688/IndB-2014-02-Helfen

Hinrichs, K. (1996). Das Normalarbeitsverhältnis und der männliche Familienernährer als Leitbilder der Sozialpolitik: Sicherungsprobleme im sozialen Wandel. Sozialer Fortschritt, 45 (4), 102-107.

Hinz, P. D. T. \& Abraham, P. D. M. (2008). Theorien des Arbeitsmarktes: Ein Überblick. In M. Abraham \& T. Hinz (Hrsg.), Arbeitsmarktsoziologie (S. 17-68). Wiesebaden: Springer VS. doi: 10.1007/978-3-531-91192-2_2

Hippel, E. von (2005). Democratizing innovation. Cambridge, Mass. [u.a.]: MIT Press.

Holst, H. (2009). Disziplinierung durch Leiharbeit? WSI-Mitteilungen, 62, 143-149.

Hondrich, K. O. \& Koch-Arzberger, C. (1992). Solidarität in der modernen Gesellschaft. Frankfurt a.M.: Fischer.

Honneth, A. (1994). Kampf um Anerkennung. Zur moralischen Grammatik sozialer Konflikte. Frankfurt a.M.: Suhrkamp.

Honneth, A. (2008). Arbeit und Anerkennung. Versuch einer Neubestimmung. Deutsche Zeitschrift für Philosophie, 56 (3), 327-341. doi: 10.1524/dzph.2008.0026

Houben, D. (2018). Von Ko-Präsenz zu Ko-Referenz - Das Erbe Erving Goffmans im Zeitalter digitalisierter Interaktion. In Leib und Netz (S. 3-20). Wiesbaden: Springer VS. doi: 10.1007/978-3-658-18863-4_1

Hyman, R. (2002). Grenzen der Solidarität. Transit. Europäische Revue, 24, 50-62.

Jansen, S. A. \& Schleissing, S. (2000). Konkurrenz und Kooperation. Interdisziplinäre Zugange zur Theorie der Co-opetitionMarburg: Metropolis.

Jemielniak, D. (2014). Common Knowledge? An Ethnography of Wikipedia. Stanford University Press. doi: 10.11126/stanford/9780804789448.001.0001

Karakayali, S. \& Moebius, S. (2014). Solidarität und Affekt. In J. Fischer \& S. Moebius (Hrsg.), Kultursoziologie im 21. Jahrhundert (S. 57-69). Wiesbaden: Springer VS. doi: 10.1007/978-3-658-03225-8_5 
Klemm, M., Kraetsch, C. \& Weyand, J. (2011). Solidarität in der europäischen betrieblichen Mitbestimmung als theoretische Herausforderung - ein kultursoziologischer Lösungsvorschlag. Industrielle Beziehungen, 18 (4), 292-314. doi: 10.1688/1862-0035_IndB_2011_04_Klemm

Klemm, M. \& Staples, R. (2015). Warten auf Antwort. In Präsenzen 2.0 (S. 113-134). Wiesbaden: Springer VS. doi: 10.1007/978-3-658-04365-0_7

Klemm, M. \& Staples, R. (Hrsg.). (2018). Leib und Netz: Sozialität zwischen Verkörperung und Virtualisierung. Wiesbaden: Springer Fachmedien. doi: 10.1007/978-3-658-18863-4

Lanier, J. (2014). Wem gehört die Zukunft? Du bist nicht der Kunde der Internetkonzerne, du bist ihr Produkt (9. Aufl). Hamburg: Hoffmann und Campe.

Müller-Jentsch, W. (1997). Soziologie der industriellen Beziehungen. Eine Einführung. Frankfurt a.M., New York: Campus.

Münch, R. \& Büttner, S. (2006). Die europäische Teilung der Arbeit. Was können wir von Emile Durkheim lernen? In M. Heidenreich (Hrsg.), Die Europäisierung sozialer Ungleichheit. Zur transnationalen Klassen- und Sozialstrukturanalyse (S. 65-107). Frankfurt a.M.: Campus.

Ostrom, E. (2008). Tragedy of the Commons. New York: Palgrave Macmillan.

Pasquier, V. \& Wood, A. J. (2018). The power of social media as a labour campaigning Tool: lessons from OUR Walmart and the Fight for 15 (ETUI Policy Brief No. 10/2018) (S. 6). ETUI.

Pfeiffer, S. (2017). Diskursmacht und technologischer Wandel. In S. Lessenich (Hrsg.), Bd. 38: Geschlossene Gesellschaften - 38. Kongress der Deutschen Gesellschaft für Soziologie. Abgerufen von http://publikationen.soziologie.de/index.php/kongressband_2016/issue/view/17

Reckwitz, A. (2012). Die Erfindung der Kreativität: Zum Prozess gesellschaftlicher Ästhetisierung. Berlin: Suhrkamp.

Reckwitz, A. (2017). Die Gesellschaft der Singularitäten: zum Strukturwandel der Moderne. Berlin: Suhrkamp.

Ringel, L. (2017). Transparenz als Ideal und Organisationsproblem: eine Studie am Beispiel der Piratenpartei Deutschland. Wiesbaden: Springer VS, Springer Fachmedien. doi: $10.1007 / 978-3-658-18328-8$

Schroeder, W., Kalass, V. \& Greef, S. (2011). Berufsgewerkschaften in der Offenvive. Vom Wandel des deutschen Gewerkschaftsmodells. Wiesbaden: VS Verlag.

Schulz, A. (2009). Strategisches Diversitätsmanagement. Unternehmensführung im Zeitalter der kulturellen Vielfalt. Wiesbaden: GWV Fachverlage. doi: 10.1007/978-3-8349-8354-1

Schütz, A. (2003). Don Quijote und das Problem der Realität. In M. Endreß \& I. Srubar (Hrsg.), Alfred Schütz Werkausgabe V.I - Theorie der Lebenswelt 1 Die pragmatische Schichtung der Lebenswelt (S. 285-324). Konstanz: UVK Verlagsgesellschaft.

Shirky, C. (2009). Here comes everybody: the power of organizing without organizations. London: Penguin Books.

Simmel, G. (1992). Soziologie - Untersuchungen über die Formen der Vergesellschaftung. Gesamtausgabe Bd.11. Frankfurt a.M.: Suhrkamp Verlag.

Stalder, F. (2013). Digital solidarity. London: Mute.

Stalder, F. (2016). Kultur der Digitalität. Berlin: Suhrkamp.

Staples, R., Trinczek, R. \& Whittall, M. (2013). „Posted Workers “: Zwischen Regulierung und Invisibilisierung. Arbeit, 22 (4), S. 271-286. doi: 10.1515/arbeit-2013-0404

Statistisches Bundesamt. (2008). Verlagerung wirtschaftlicher Aktivitäten - Ergebnisse der Piloterhebung (S. 56). Wiesbaden.

Stichweh, R. (2000). Die Weltgesellschaft: soziologische Analysen. Frankfurt a.M.: Suhrkamp.

Thijssen, P. (2012). From mechanical to organic solidarity, and back: With Honneth beyond Durkheim. European Journal of Social Theory, 15 (4), 454-470. doi: 10.1177/1368431011423589 
Turkle, S. (2011). Alone together: Why We Expect More from Technology and Less from Each Other. New York: Basic Books.

Voß, G. G. \& Pongratz, H. J. (1998). Der Arbeitskraftunternehmer. Eine neue Grundform der „Ware Arbeitskraft“"? Kölner Zeitschrift für Soziologie und Sozialpsychologie, 50 (1), 131-158.

Voswinkel, S. (2012). Verstrebungen der Fluidität. Ansprüche von Beschäftigten und Politiken von Organisationen im Wandel der Anerkennungsverhältnisse. Industrielle Beziehungen, 19 (4), S. 412-431. doi: 10.1688/1862-0035_IndB_2012_04_Voswinkel

Windeler, A. (2012). Kooperation und Konkurrenz in Netzwerken. Theoretische Überlegungen zur Analyse des Strukturwandels der Arbeitsorganisation. In C. Schilcher, M. Will-Zocholl \& M. Ziegler (Hrsg.), Vertrauen und Kooperation in der Arbeitswelt (S. 23-50). Wiesbaden: Springer VS. doi: 10.1007/978-3-531-94327-5_2

Zoll, R. (2000). Was ist Solidarität heute? Frankfurt a.M.: Suhrkamp. 


\section{ILERA European Congress 2019}

\section{Perspectives of Employment Relations in Europe, Düsseldorf, Germany, 5-7 September 2019}

(http://ilera2019.giraweb.de/)

(www.ilera2_019.eu)

\section{CALL FOR PAPERS AND WORKSHOP PROPOSALS (deadline: 20 December 2018)}

The XII European Regional Congress of the International Labour and Employment Relations Association (ILERA) will take place in Düsseldorf on 5-7 September 2019 and intends to explore and analyse the changes in labour and employment relations across Europe and in a comparative perspective.

Stable labour relations and welfare state protections have been perceived to be at the core of what has been named the European social model. Beginning in the 1990s, however, their foundations seem to have been weakened in many countries: We witness a decline in bargaining cover- age, employer and union densities but also many efforts to restructure the welfare state as well as systems of labour market regulation. The 12th ILERA European Congress comes at a time when changes of institutions, actors and practices of labour relations are widespread. We expect recent current events such as the digitalisation of the economy, intensified conflict on trade, the exit of the United Kingdom from the EU as well as the vastly changing political landscape which comes along with the rise of right-wing populist parties to influence such changes in various ways. For good or bad, industrial relations will not remain the same. We invite participants to contribute towards analysing the direction, content and speed of such processes of change but also the current state of labour relations in Europe and beyond. We are particularly interested in receiving paper proposals which engage in research on how societal actors seek to actively shape labour relations, institutions, processes and outcomes.

Papers and workshops addressing the multi-faceted issues outlined above are welcome. In particular, proposals can refer to the following four tracks:

\section{Track 1: Social Europe: Equality and Poverty}

The idea of Social Europe is widely associated with strong labour market institutions and employment relations which largely contribute to comparatively high levels of social protection and low inequality. Trust in European institutions and the commitment to build a better future are not necessarily taken for granted as the rise of populist parties in many European countries seems to challenge the European idea. In addition, during recent decades Europe has been faced by growing social and economic disparities both within and between regions and states but also by persistent gaps between sexes as well as between migrant and native workers. Although the reasons for growing inequalities are complex and manifold, 
changes in labour market institutions and the power relations of the social partners are widely regarded as one major cause for this development. Therefore, Track 1 will tackle the following issues:

- Influence of labour market institutions and employment relations on the different dimensions of inequality (income, wealth, training and employment opportunities etc.)

- Contributions of social partners to a reduction of inequality and to a more inclusive economic development

- Employment relations and macroeconomic performance

- New forms and concepts of labour market regulation and employment relations at European level

- EU economic governance and industrial relation

- Mobility of labour in Europe

- Contentious politics in Europe: protest, opposition, and mobilisation

\section{Track 2: Regulation of Labour: Actors, Institutions and Law}

The regulation of labour is a dynamic process subject to power relations in public policies and collective bargaining. This track aims at gathering contributions on the policies, the practice and enforcement of labour legislation and collective agreements.

The past decade has seen a growing impact of the supranational level as European policies have aimed at influencing labour and employment policies in the member states. Simultaneously, at member state level, nationalst political parties with divergent policies on labour institutions but similar opposition to foreign workers have grown. This happens against the background of a declining membership strength of trade unions and employer organisations, policies promoting a decentralisation of collective bargaining and opposition to the IR system by digital multinationals.

We are interested in papers and workshops on:

- The relationship between law and other forms of regulation and between "soft" and "hard" law

- Conflicts caused by the relationship between national and supra-national labour law

- Enforcement of labour regulation - enforcing institutions and actors; strategies for transnational enforcement of labour rights

- The role of courts and tribunals as actors in labour relations

- Development of actors, trade unions and employer organisations, at national and EU level

- Transnational regulation of labour relations and the regulation of cross-border mobility

- Nationalist party policies and the regulation of labour

- Regulation of the digital economy and the role of digital multinationals

\section{Track 3: Workers' Voice and Industrial Democracy}

Europe is the origin of a rich variety of different concepts of industrial and workplace democracy. For decades the incompatibility of these traditions was more an obstacle than a fertile ground for a strong European representation of workers' voice. Meanwhile considerable progress has been made regarding workers' participation in Europe as well as beyond. Yet democracy is threatened at different levels and from different sides, in particular by the rise of authoritarian political movements and new forms of racism, sexism, classism and religious 
fundamentalism that also affect workplace relations. At the same time new technologies lead to new challenges as well as opportunities for democratic participation. Against this background we want to discuss the future of democratic participation at local, national, European and international levels.

We welcome papers on:

- Concepts and traditions: industrial democracy, industrial citizenship, 'autogestion" 'Mitbestimmung" (co-determination) and beyond

- Debate about European minimum standards for board-level employee representation

- Workers' voice in transnational Companies: European works councils, employee involvement in European Companies (SE), International Framework Agreements

- Union busting in international comparison

- Voluntary forms of employee participation

- Direct and representative participation

- Economic outcomes of workers' participation

- Fragmentation of the workforce as a challenge for democratic participation

- New solidarities and new forms of participation

- Gender and diversity in labour unions and works councils

\section{Track 4: Human Resources, Quality of Work and Digitalisation}

HRM is a continuously changing practice - not only in the firm, but also in interorganisational relations between firms or between firms and (self-employed) individuals. As a consequence, we experience a massive change in practices of HRM, in the respective constellations of actors in HRM and employment relations. A growing digitalisation of (multinational) firms as well as their business relationships, new business models based on digital technologies (e.g., crowdwork) leading to a "Gig economy" and the use of artificial intelligence impacts on the quality of work, HRM practices and employment relations.

Under this track, we welcome empirical, theoretical as well as comparative papers and workshops on, for example:

- $\quad$ HRM, quality of work and working conditions

- Consequences of digitalisation for HRM policy fields and employment relations

- New developments in HRM, for example talent analytics or automatic decision making

- New practices in the policy fields of HRM in and beyond the single firm

- Intermediated work

- HRM for highly-skilled workers and low-skilled workers

We are also looking forward to papers which discuss these topics with reference to gender, migrant and disabled workers.

\section{Abstracts are invited for Submission through the congress website at:}

www.ilera2019giraweb.de/cfp

Deadline for abstracts: 20 December 2018

Notifications of acceptance will be sent by 28 February 2019 Registration for the Congress will start on 31 January 2019 
Industrielle Beziehungen, Jahrgang 25, Heft 4, 2018 IndBez 25(4)

\title{
Industrielle Beziehungen \\ die nächsten Themenschwerpunkte
}

\begin{abstract}
Arbeitswelten und Arbeitsbeziehungen im digitalisierten Betrieb
Herausgeber: Stefan Kirchner und Wenzel Matiaske

Sorgearbeit und Industrielle Beziehungen

HerausgeberInnen: Ingrid Artus, Peter Birke, Stefan Kerber-Clasen, Wolfgang Menz
\end{abstract}
Arbeitswelten und Arbeitsbeziehungen in der Plattformökonomie Herausgeber: Stefan Kirchner und Wenzel Matiaske

\begin{tabular}{|c|c|c|}
\hline Knud Andresen & Thomas Haipeter & Tanja Paulitz \\
\hline Phillip Balsiger & Heiner Heiland & Jessica Pflüger \\
\hline Martin Behrens & Nicole Helmerich & Ludger Pries \\
\hline Ingo Bode & Julia Hofmann & Jürgen Prott \\
\hline Kendra Briken & Hajo Holst & Katja Rost \\
\hline Ulrich Brinkmann & Stefanie Hürtgen & Stefan Rueb \\
\hline Florian Butollo & Berndt Keller & Sebastian Schief \\
\hline Bernhard Ebbinghaus & Stefan Kerber-Clasen & Kai-Uwe Schnapp \\
\hline Peter Ellguth & Hermann Kotthoff & Elke Schüßler \\
\hline Roland Erne & Steffen Lehndorf & Eva Senghaas-Knobloch \\
\hline Carola Frege & Steffen Liebig & Verena Tobsch \\
\hline Gerd Grözinger & Katharina Liebsch & Claudius Wagemann \\
\hline Kevin Guillascavan & Antje Mohr & Manfred Wannoeffel \\
\hline
\end{tabular}




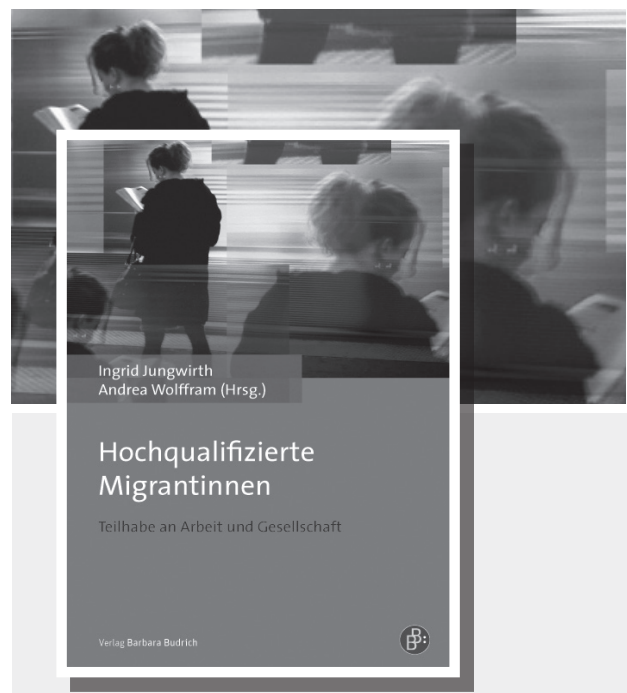

Ingrid Jungwirth

Andrea Wolffram (Hrsg.)

\section{Hochqualifizierte Migrantinnen}

\section{Teilhabe an Arbeit und Gesellschaft}

Die Autorlnnen des Sammelbands untersuchen, wie sich hochqualifizierte Migrantinnen in den deutschen Arbeitsmarkt integrieren und unter welchen Bedingungen sie ihre Qualifikationen umsetzen und nutzen können. Ein zentrales Anliegen ist es, Geschlecht als analytische Dimension in die Untersuchung hochqualifizierter Migration systematisch einzubeziehen.

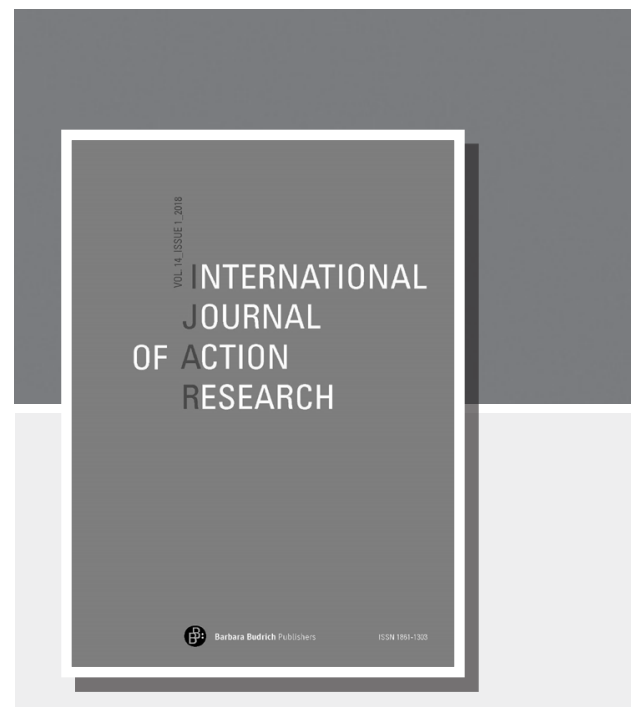

\section{IJAR - International Journal of Action Research}

IJAR - International Journal of Action Research provides a forum for an open and non dogmatic discussion about action research, regarding both its present situation and future perspectives. This debate is open to the variety of action research concepts worldwide. The focus is on dialogues between theory and practice. IJAR is a refereed journal and published three times a year.

Volume 14, 2018 • approx. 100 pp. per issue • English ISSN 1861-1303 • elSSN 1861-9916 